\title{
Health aberrations in children with overweight and obesity
}

Citation for published version (APA):

Karnebeek, K. (2020). Health aberrations in children with overweight and obesity: a special focus on the liver. [Doctoral Thesis, Maastricht University]. Gildeprint en Universitaire Pers Maastricht. https://doi.org/10.26481/dis.20200109kk

Document status and date:

Published: 01/01/2020

DOI:

$10.26481 /$ dis.20200109kk

Document Version:

Publisher's PDF, also known as Version of record

\section{Please check the document version of this publication:}

- A submitted manuscript is the version of the article upon submission and before peer-review. There can be important differences between the submitted version and the official published version of record.

People interested in the research are advised to contact the author for the final version of the publication, or visit the DOI to the publisher's website.

- The final author version and the galley proof are versions of the publication after peer review.

- The final published version features the final layout of the paper including the volume, issue and page numbers.

Link to publication

\footnotetext{
General rights rights.

- You may freely distribute the URL identifying the publication in the public portal. please follow below link for the End User Agreement:

www.umlib.nl/taverne-license

Take down policy

If you believe that this document breaches copyright please contact us at:

repository@maastrichtuniversity.nl

providing details and we will investigate your claim.
}

Copyright and moral rights for the publications made accessible in the public portal are retained by the authors and/or other copyright owners and it is a condition of accessing publications that users recognise and abide by the legal requirements associated with these

- Users may download and print one copy of any publication from the public portal for the purpose of private study or research.

- You may not further distribute the material or use it for any profit-making activity or commercial gain

If the publication is distributed under the terms of Article $25 \mathrm{fa}$ of the Dutch Copyright Act, indicated by the "Taverne" license above, 
Health aberrations in children with overweight and obesity:

a special focus on the liver 
(C) Copyright Kylie Karnebeek, 2020, Maastricht

ISBN: 9789463239523

Cover design: Kylie Karnebeek

Lay-out: Kylie Karnebeek

Printed by: Gildeprint

The research presented in this thesis was conducted within the Centre for Overweight Adolescent and Children's Healthcare, Department of Pediatrics, Maastricht University Medical Centre+ and the School of Nutrition and Translational Research in Metabolism, Maastricht University. 


\title{
Health aberrations in children with overweight and obesity: a special focus on the liver
}

\author{
PROEFSCHRIFT \\ Ter verkrijging van de graad van doctor aan de Universiteit Maastricht, \\ op gezag van de Rector Magnificus, Prof. dr. Rianne M. Letschert \\ volgens het besluit van het College van Decanen, \\ in het openbaar te verdedigen op \\ donderdag 9 januari 2020 om 12.00 uur.
}

door

Kylie Karnebeek

Geboren op 13 april 1990 te Heerlen 


\section{Promotoren}

Dr. A.C.E. Vreugdenhil

Prof. dr. J. Plat

Prof. dr. L.J.I. Zimmermann

\section{Beoordelingscommissie}

Prof. dr. S.P.J. Kremers (voorzitter)

Prof.dr.ir. E.J.M. Feskens (Wageningen University)

Dr. G. Koek

Prof. dr. P. Schrauwen

Prof.dr. H.J. Verkade (Universitair Medisch Centrum Groningen) 


\section{Contents}

Chapter 1

General introduction

7

Chapter 2 Comorbidities in primary versus secondary school children with obesity and responsiveness to lifestyle intervention

Chapter 3 Changes in free-living glycemic profiles after 12 months of lifestyle intervention in children with overweight and obesity

Chapter 4 Predictors of arterial stiffness in children with overweight, obesity and morbid obesity are sex-specific

Chapter 5 A narrative review of factors associated with development and progression of non-alcoholic fatty liver disease

Chapter 6 Factors associated with increasing degrees of hepatic steatosis 101 and progression to steatohepatitis in children with overweight or obesity

Chapter 7 Genotype-specific determinants of alanine aminotransferase levels in children with overweight and obesity

Chapter $8 \quad$ Low levels of antibodies against oxidation specific epitopes in oxLDL are associated with parameters reflecting NAFLD and metabolic health in children

Valorisation

Dankwoord

About the author 

Chapter 1

General introduction 


\section{Childhood overweight and obesity}

The global prevalence of overweight and obesity in children and adults increased tremendously during the past decades, nowadays reaching epidemic proportions (1). Particularly, the high prevalence of overweight and obesity in children needs attention, since it can be envisioned that the consequences of overweight and obesity can be influential especially at a young age. In 2015, it was estimated that approximately 108 million children worldwide had obesity (1). In Western Europe, the prevalence of obesity in boys and girls younger than 20 years is $7.2 \%$ and $6.4 \%$ respectively (2). In 2018, the prevalence of overweight in children aged 4 to 17 years in the Netherlands was $8.9 \%$ in boys and $9.1 \%$ in girls, while the prevalence of obesity was $2.5 \%$ in boys and $3.0 \%$ in girls (3).

In almost all cases, the development of overweight and obesity is the consequence of a persistent imbalance between energy intake and energy expenditure, which are influenced by intrinsic characteristics of the child (for instance, familial susceptibility to weight gain, age and sex), but also by familial characteristics (for instance, parents' knowledge and encouragement of healthy lifestyle) and societal characteristics (for example, socioeconomic status, food and physical activity programs in schools)(4).

In general, underlying physical conditions (e.g. endocrine problems, syndromes or monogenetic obesity) cause or contribute to the development of obesity in only a very small percentage of children. However, the prevalence of underlying physical conditions can be higher in certain subpopulations. For instance, a recent Dutch study described monogenetic or syndromic obesity in $7.3 \%$ of children with severe early onset obesity (5).

\section{Consequences of obesity}

Obesity is an important risk factor for the development of comorbidities affecting various organ systems. Numerous studies have shown that the early development of these comorbidities can already start from a very young age in children with overweight or obesity. Obesity is associated with presence of metabolic disturbances, such as elevated lipid and lipoprotein concentrations $(6,7)$, insulin resistance $(8)$, low-grade systemic inflammation (9) and oxidative stress. These aberrations play an important role in the development of obesity-related diseases, including cardiovascular disease, diabetes and sleep apnea, but also non-alcoholic fatty liver disease (NAFLD) and kidney disease $(6,10$ 12). Children with obesity are likely to grow up to become adults with overweight or obesity and obesity-related health aberrations during childhood are also strong predictors 
for disease at adult age (13). An overview of obesity-related diseases in children and adults are shown in figure 1.

In addition to these health effects, obesity in adults has also been associated with an increased all-cause mortality (14), decreased quality of life (15), lower work productivity (16) and an increased utilization of healthcare services and higher healthcare costs (17). These findings demonstrate that obesity does not merely impact the life of the individual, but also society. A societal cost-benefit analysis of a two-year multidisciplinary intervention aimed at improving the lifestyle of children with overweight and obesity (the Gezond Onderweg! intervention in Arnhem, the Netherlands) estimated that the societal benefits of such an intervention are 4-7 higher than the societal costs. The benefits of the approach were mainly due to an improvement of quality of life because of a decreased disease burden, and decreased healthcare costs (18).
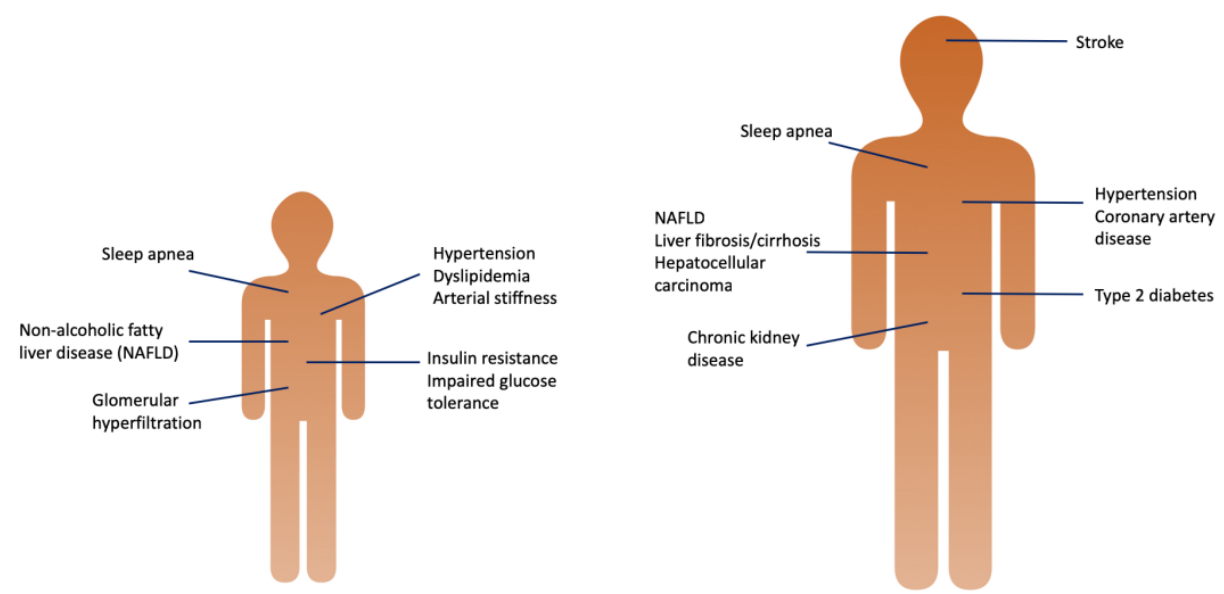

Figure 1. An overview of early stages of obesity-related diseases in childhood and severe stages of obesityrelated diseases in adulthood.

\section{Definition and pathophysiology non-alcoholic fatty liver disease}

Nonalcoholic fatty liver disease is a chronic liver disease and encompasses a spectrum of liver disorders ranging from hepatic steatosis, to steatohepatitis (NASH), liver fibrosis and cirrhosis. Liver steatosis is defined as a hepatic triglyceride content of more than $5 \%$, in the absence of other liver diseases and/or excessive alcohol consumption and is usually 
considered a benign and reversible condition. In some patients, liver steatosis will progress to $\mathrm{NASH}$, a fatty and inflamed liver with injury to the hepatocytes. Just like simple steatosis, NASH is a reversible condition. In a small percentage of NASH patients, the disease will progress to liver fibrosis and possibly even liver cirrhosis, which is considered irreversible. When liver cirrhosis leads to end-stage liver failure, a liver transplant will become necessary (19). With the increasing prevalence, NAFLD has now become second leading cause of liver transplantation in the United States (20). Additionally, patients with NAFLD, also those without liver cirrhosis, have an increased risk of developing hepatocellular carcinoma $(21,22)$.

In a large meta-analysis from 2016, the global prevalence of hepatic steatosis in adults was estimated to be $25 \%$, with an increasing prevalence with age (23). Approximately $50 \%$ of these adults with hepatic steatosis was obese. In this meta-analysis, the prevalence of NASH was estimated to be 1.5-6.5\%, depending on the indication for liver biopsy. Approximately $80 \%$ of the patients with NASH was obese (23). Another study in adults has estimated that cirrhosis may develop in around $20 \%$ of patients with NASH (24). Progression to the severe end of the disease spectrum is much more rapid in some patients compared to others.

NAFLD is already common at a young age, with an estimated prevalence of hepatic steatosis of $34 \%$ in pediatric patients from obesity clinics, compared to $8 \%$ in children from the general population (25). Goyal et al. have estimated that $25-50 \%$ of children with NAFLD have steatohepatitis at time of diagnosis and $10-25 \%$ have liver fibrosis. These percentages were based on available studies in children that mainly underwent a liver biopsy based on clinical considerations (26). The natural history of NAFLD in children still remains to be elucidated, since longitudinal studies are limited. A previous study by Feldstein et al. studied longitudinal data (up to 20 years) of a small group of children with biopsy-proven NAFLD (27). Of the 29 children in this study that underwent a liver biopsy, 17 children already had some degree of liver fibrosis at presentation. One child presented with NASH without liver fibrosis, but progressed to stage four liver fibrosis with cirrhosis within 5 years. Two children had to undergo a liver transplant due to severe liver cirrhosis before the age 25 years (27). A-Kader et al. studied 18 children with biopsy-proven NAFLD that underwent a repeated biopsy 24-39 months later. The authors found progression of fibrosis in 7 children, stable fibrosis in 8 children, regression of fibrosis in 1 child and complete resolution of steatosis and fibrosis in 2 children that lost weight (28). Even though long-term follow-up studies are needed to elucidate the natural history and progression in children with NAFLD, these results demonstrate that progression to more severe stages of the disease can already be seen at a young age in a certain subgroup of 
children. These results show that NAFLD in children should not be underestimated and underline the importance of gaining more insight into the risk factors for rapid progression to more severe disease states.

Currently, the underlying pathophysiological mechanisms of NAFLD development and progression are still incompletely understood, but it is clear that it is the consequence of a complex interplay of multifactorial processes. As previously mentioned, the obesity epidemic is assumed to be an important driving factor behind the increasing NAFLD prevalence. Common metabolic aberrations in overweight and obesity such as insulin resistance, elevated triglyceride levels and hypertension have all been associated with NAFLD (29-31). However, it remains to be clarified whether metabolic aberrations precede the development of NAFLD or whether the association is vice versa. Factors such as gut barrier dysfunction (32), obesity-related low-grade inflammation (33) and oxidative stress $(34,35)$ have been associated with progression to more severe disease states.

Moreover, in addition to the aforementioned factors, that are often related to obesity, genetics, age, sex and ethnicity have also been associated with the development of NAFLD. Several single nucleotide polymorphisms, amongst which the PNPLA3 I148M and TM6SF2 E167K variants, have been identified that increase the risk of NAFLD development and may contribute to progression of the disease $(36,37)$. A previous study in twins has estimated that the heritability of hepatic steatosis is approximately $50 \%(38)$, showing that genetic predisposition is a large contributor to the development of NAFLD. Moreover, NAFLD is more common in males than in females, at least during reproductive age (39). Finally, ethnical background plays a role in NAFLD risk, with the highest prevalence in people from South-American and Middle Eastern descent (23).

A very important aspect in understanding the pathophysiology of NAFLD development and progression in children is that liver biopsy studies have shown that histological characteristics in children with NASH differ from the characteristics in adults with NASH, suggesting that underlying pathophysiological processes might differ between children and adults. Also, it underlines the importance of studying NAFLD and NASH in children. An overview of the different histological characteristics of NASH in children and adults is shown in figure 2. Moreover, a summary of the NAFLD disease spectrum and associated factors throughout life can be seen in figure 3 . 


\begin{tabular}{|c|c|c|}
\hline & $\begin{array}{l}\text { Histologic characteristics seen mainly in } \\
\text { preadolescent children }\end{array}$ & $\begin{array}{l}\text { Histologic characteristics seen mainly in } \\
\text { older adolescent children and adults }\end{array}$ \\
\hline Steatosis & Zone 1 (periportal) to panacinar distribution & $\begin{array}{l}\text { Zone } 3 \text { (pericentral/perivenous) to panacinar } \\
\text { distribution }\end{array}$ \\
\hline Inflammation & $\begin{array}{l}\text { Portal inflammation equal to or greater than } \\
\text { lobular inflammation in early disease }\end{array}$ & $\begin{array}{l}\text { Lobular inflammation generally more } \\
\text { prominent than portal inflammation in early } \\
\text { disease }\end{array}$ \\
\hline Ballooning & Indistinct or absent & Present, usually in zone 3 \\
\hline Fibrosis & $\begin{array}{l}\text { Periportal fibrosis to portal-portal bridging } \\
\text { fibrosis }\end{array}$ & $\begin{array}{l}\text { Perisinusoidal fibrosis in zone } 3 \text { to portal- } \\
\text { central bridging fibrosis }\end{array}$ \\
\hline
\end{tabular}

Figure 2. An overview of the different histological characteristics of NASH in children and adults, adapted from Kleiner et al. (40).

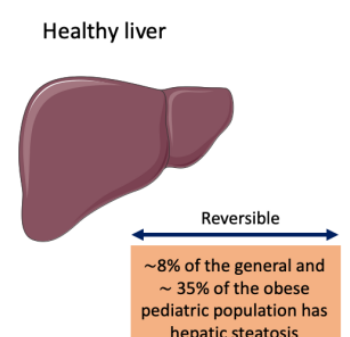

hepatic steatosis
Hepatic steatosis

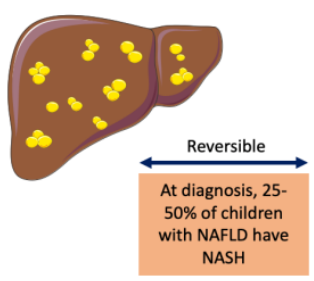

Fatty and inflamed liver (NASH), with or without fibrosis

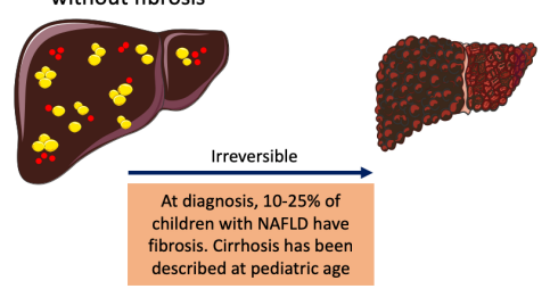

Associated factors throughout life

Genetic predisposition, ethnicity, maternal factors

Male sex (from puberty) , obesity, (cardio)metabolic aberrations, inflammation, oxidative stress, lifestyle habits

Age, female sex (from menopause on)

Figure 3. A summary of the NAFLD disease spectrum and the associated risk factors throughout life. 


\section{Consequences and treatment of non-alcoholic fatty liver disease}

It is important not to underestimate the health risk that is associated with NAFLD. Besides the fact that NAFLD has been associated with an increased risk of developing hepatocellular carcinoma (41), severe progressive NAFLD (i.e. liver cirrhosis) may eventually necessitate a liver transplantation (20). Moreover, NAFLD has also been identified as an independent risk factor for the development of diabetes type II (42) and cardiovascular disease (43). These findings stress the need for prevention and early interventions.

Therapeutic options to treat NAFLD are limited, despite ongoing research efforts to identify new options targeting different processes involved in NAFLD/NASH pathophysiology $(44,45)$. Currently, the recommended treatment for children with NAFLD according to the North American Society of Pediatric Gastroenterology, Hepatology and Nutrition guideline is decreasing obesity by lifestyle improvements (46). A previous study in adults has shown that loss of $7-10 \%$ of body weight already improves histological abnormalities of NASH (47). A recent review of pediatric studies has suggested that lifestyle improvements in children can also improve surrogate markers of NAFLD, even in children without a significant decrease in BMI z-score (48). However, lifestyle improvements and weight loss can be difficult to achieve and especially difficult to maintain without guidance. Therefore, in some patients with (morbid) obesity and severe NAFLD, that were not able to lose weight through lifestyle changes, bariatric surgery might be necessary to achieve weight loss. Bariatric surgery in an adult study led to a resolution of NASH after one year in the majority of patients (49). These findings underline the importance of interventions to help children in realizing a healthier lifestyle.

\section{Diagnosing non-alcoholic fatty liver disease}

NAFLD is often asymptomatic and most patients do not have specific symptoms until they have progressed to the severe stages of the disease spectrum. Currently, the gold standard for diagnosing nonalcoholic fatty liver disease is a liver biopsy. However, this is an invasive procedure with a small risk of severe complications making it unsuitable for screening the large number of people that are at-risk for developing NAFLD. ${ }^{1} \mathrm{H}$ magnetic resonance spectroscopy $\left({ }^{1} \mathrm{H}-\mathrm{MRS}\right)$ is a noninvasive technique that was shown to have a high sensitivity to quantitatively measure hepatic fat content, but not the presence of steatohepatitis and/or liver fibrosis. Costs and the requirement of specific equipment that is not readily available, make ${ }^{1} \mathrm{H}-\mathrm{MRS}$ also impractical for screening at-risk populations for the presence of fatty liver. Ultrasonography is an inexpensive, readily available method to estimate whether or not a patient has (severe) steatosis. However, previous studies have shown that qualitative ultrasonography has limited sensitivity to diagnose especially mild hepatic steatosis (50). The ultrasonographic hepatorenal index (HRI) is an easy, noninvasive and sensitive measurement to quantitatively asses hepatic steatosis. Previous studies in adults have shown a very high correlation between the HRI and liver fat content in liver biopsies $(51,52)$ and liver fat as measured with ${ }^{1} \mathrm{H}$-MRS $(53)$. Studies comparing the HRI with other diagnostic options have not yet been performed in children. However, 
since the HRI measurement is based on calculating the ratio of the echogenicity of the liver and the kidney, it seems unlikely that the comparability with other diagnostics would differ between children and adults.

However, like ${ }^{1} \mathrm{H}-\mathrm{MRS}$, the HRI is also not able to identify the presence of steatohepatitis and/or liver fibrosis. Alanine aminotransferase (ALT) is released into the blood as a consequence of hepatocellular injury (54) and can be elevated as a consequence of several (chronic) liver diseases (55). In the absence of other liver disease (e.g. viral hepatitis, metabolic or toxic liver disease) and in the presence of fatty liver, ALT levels can be seen as a surrogate marker for steatohepatitis. Research towards novel, highly-sensitive biomarkers that are more specific for NASH are still undergoing.

\section{Lifestyle intervention}

Lifestyle interventions have previously shown to be effective in decreasing BMI z-score in children with obesity. Also, studies have shown that lifestyle interventions are more effective in reducing BMI z-score in younger children than in adolescents (56) and that younger patients were more likely to maintain weight loss (57). Moreover, even though most lifestyle intervention studies focus on BMI z-score as the main outcome, previous studies have shown that comorbidities, like hypertension, insulin resistance and dyslipidemia, can also improve during the intervention $(58,59)$. All studies described in this thesis were performed at the Centre for Overweight Adolescent and Children's Healthcare (COACH) in the Maastricht UMC+. The COACH program is an interdisciplinary, long-term, outpatient, family-based lifestyle intervention, provided by a team of physicians, dieticians, a psychologist, a pedagogue, nurses and physical activity coach. All children underwent a comprehensive assessment before the start of the intervention, to exclude underlying syndromic or endocrine conditions of obesity, to evaluate health complications and risk factors, and to gain insight in behavior and (family) functioning. A follow-up assessment including all the examinations performed during the initial assessment was offered to all children after each year of lifestyle intervention. Information gathered in this assessment was used to develop a care plan that was tailored to the needs of each family. All children and their families were offered individual guidance focusing on lifestyle improvements with regards to nutrition, food habits, physical activity, sleep and psychological and social aspects. Points of improvement that were identified during the initial assessment were used as focus points for making small, step-by-step lifestyle changes. These focus points were adapted throughout the intervention, depending on for instance the successfulness of making changes or the identification of new points of improvements. In addition to the individual visits, group activities related to nutrition and physical activity were organized several times a year. Children, that were not already participating in regular sport activities, were encouraged to participate in a weekly one-hour physical activity group lesson. 


\section{Aims and outline of this thesis}

The aims of these studies were 1) to assess the health consequences of overweight and obesity in children in general, 2) to gain more insight into determinants and risk factors of hepatic steatosis and steatohepatitis in children with overweight and obesity and 3) to evaluate the effects of one year of the $\mathrm{COACH}$ lifestyle intervention on BMI z-score and comorbidities.

In chapter 2, we evaluated the prevalence of comorbidities in school-aged children with obesity, including NAFLD, but also metabolic, cardiovascular and renal health. In this chapter, we also studied the effect of one year of the $\mathrm{COACH}$ lifestyle intervention in primary and secondary school children.

In chapter 3, we have focused in particular on the effect of one year COACH lifestyle intervention on glycaemic profiles measured in free-living conditions in a small group of children with overweight and obesity. In this chapter we also evaluated the association between changes in glycaemic profiles and changes in cardiovascular risk parameters.

In addition to the cardiovascular health outcomes that were studied in chapter 2, we focused in more detail on the augmentation index, as a marker for arterial stiffness, in chapter 4 . In this study, we evaluated the associations between the augmentation index and markers for inflammation and endothelial dysfunction, in addition to classical cardiovascular risk parameters in children with overweight, obesity and morbid obesity.

Since the underlying mechanisms of NAFLD are still not fully elucidated, we have studied factors that contribute to (progressive) NAFLD in children with overweight or obesity.

In chapter 5, we have reviewed the current literature regarding pathophysiological processes and determinants that contribute to NAFLD development and progression in children.

In chapter 6, we aimed to identify determinants of increasing degrees of hepatic steatosis, as estimated with the ultrasonographic hepatorenal index (HRI), in children with overweight, obesity and morbid obesity, and subsequently to identify which factors contribute to progression to steatohepatitis, as measured with alanine aminotransferase (ALT) levels.

During the past decade, it has become clear that genetic predisposition is an important risk factor for NAFLD. Since the genetic variants that can contribute to NAFLD development concern variants in genes encoding proteins involved in different processes of NAFLD development, it can be hypothesized that factors associated with NAFLD might be genotype and SNP specific. Therefore, in chapter 7, we evaluated if the determinants of ALT levels in children with overweight differ depending on genetic variants.

Previous studies have shown LDL cholesterol can become oxidized under the influence of oxidative stress. Studies in adults have shown that this oxidized LDL plays a role in the 
development of NAFLD. Antibodies against oxLDL are thought to be protective against this process. In chapter 8, we evaluated associations between circulating concentrations of antibodies against oxidized LDL and parameters reflecting NAFLD. Additionally, we aimed to assess if these associations were liver-specific by also evaluating the associations between the antibodies and parameters reflecting glucose metabolism, systemic inflammation and cardiovascular health.

Finally, the general discussion in chapter 9 summarizes the major findings of the studies presented in this thesis, discusses these results in a broader perspective and offers future perspectives. 


\section{References}

1. Afshin A, Forouzanfar MH, Reitsma MB, Sur P, Estep K, Lee A, et al. Health Effects of Overweight and Obesity in 195 Countries over 25 Years. The New England journal of medicine. 2017;377(1):13-27.

2. Ng M, Fleming T, Robinson M, Thomson B, Graetz N, Margono C, et al. Global, regional, and national prevalence of overweight and obesity in children and adults during 19802013: a systematic analysis for the Global Burden of Disease Study 2013. Lancet (London, England). 2014;384(9945):766-81.

3. National Institute of Public Health and the Environment (Rijksinstituut voor Volksgezondheid en Milieu; RIVM). Website: https://www.volksgezondheidenzorg.info/onderwerp/overgewicht/cijferscontext/huidige-situatie\#!node-overgewicht-kinderen. Accessed: 05/07/2018.

4. Davison KK, Birch LL. Childhood overweight: a contextual model and recommendations for future research. Obesity reviews : an official journal of the International Association for the Study of Obesity. 2001;2(3):159-71.

5. Kleinendorst L, Massink MPG, Cooiman MI, Savas M, van der Baan-Slootweg OH, Roelants RJ, et al. Genetic obesity: next-generation sequencing results of 1230 patients with obesity. Journal of medical genetics. 2018;55(9):578-86.

6. Skinner AC, Perrin EM, Moss LA, Skelton JA. Cardiometabolic Risks and Severity of Obesity in Children and Young Adults. The New England journal of medicine. 2015;373(14):130717.

7. Martin L, Oepen J, Reinehr T, Wabitsch M, Claussnitzer G, Waldeck E, et al. Ethnicity and cardiovascular risk factors: evaluation of 40,921 normal-weight, overweight or obese children and adolescents living in Central Europe. Int J Obes (Lond). 2015;39(1):45-51.

8. Shashaj B, Luciano R, Contoli B, Morino GS, Spreghini MR, Rustico C, et al. Reference ranges of HOMA-IR in normal-weight and obese young Caucasians. Acta diabetologica. 2016;53(2):251-60.

9. Visser M, Bouter LM, McQuillan GM, Wener MH, Harris TB. Low-grade systemic inflammation in children. Pediatrics. 2001;107(1):E13.

10. Perrone J, Hollander JE, De Roos F. Cardiovascular risk factors and atherosclerosis in children and young adults. The New England journal of medicine. 1998;339(15):1083-4.

11. Corey KE, Kaplan LM. Obesity and liver disease: the epidemic of the twenty-first century. Clinics in liver disease. 2014;18(1):1-18.

12. Herouvi D, Karanasios E, Karayianni C, Karavanaki K. Cardiovascular disease in childhood: the role of obesity. Eur J Pediatr. 2013;172(6):721-32.

13. Juhola J, Magnussen CG, Viikari JS, Kahonen M, Hutri-Kahonen N, Jula A, et al. Tracking of serum lipid levels, blood pressure, and body mass index from childhood to adulthood: the Cardiovascular Risk in Young Finns Study. The Journal of pediatrics. 2011;159(4):584-90.

14. Flegal KM, Kit BK, Orpana H, Graubard BI. Association of all-cause mortality with overweight and obesity using standard body mass index categories: a systematic review and meta-analysis. JAMA. 2013;309(1):71-82.

15. Tsiros MD, Olds T, Buckley JD, Grimshaw P, Brennan L, Walkley J, et al. Health-related quality of life in obese children and adolescents. Int J Obes (Lond). 2009;33(4):387-400.

16. Hammond RA, Levine R. The economic impact of obesity in the United States. Diabetes, metabolic syndrome and obesity : targets and therapy. 2010;3:285-95.

17. Kamble PS, Hayden J, Collins J, Harvey RA, Suehs B, Renda A, et al. Association of obesity with healthcare resource utilization and costs in a commercial population. Current medical research and opinion. 2018:1-18. 
18. Harchaoui S, Vellenga I, Spit W, Steeg van de L, Peters N. Maatschappelijke effecten van aanpak van obesitas - Kosten en baten van Gezond Onderweg! 2018.

19. Mikolasevic I, Filipec-Kanizaj T, Mijic M, Jakopcic I, Milic S, Hrstic I, et al. Nonalcoholic fatty liver disease and liver transplantation - Where do we stand? World journal of gastroenterology. 2018;24(14):1491-506.

20. Wong RJ, Aguilar M, Cheung R, Perumpail RB, Harrison SA, Younossi ZM, et al. Nonalcoholic steatohepatitis is the second leading etiology of liver disease among adults awaiting liver transplantation in the United States. Gastroenterology. 2015;148(3):547-55.

21. Degasperi $E$, Colombo M. Distinctive features of hepatocellular carcinoma in non-alcoholic fatty liver disease. The lancet Gastroenterology \& hepatology. 2016;1(2):156-64.

22. Perumpail RB, Wong RJ, Ahmed A, Harrison SA. Hepatocellular Carcinoma in the Setting of Non-cirrhotic Nonalcoholic Fatty Liver Disease and the Metabolic Syndrome: US Experience. Digestive diseases and sciences. 2015;60(10):3142-8.

23. Younossi ZM, Koenig AB, Abdelatif D, Fazel Y, Henry L, Wymer M. Global epidemiology of nonalcoholic fatty liver disease-Meta-analytic assessment of prevalence, incidence, and outcomes. Hepatology (Baltimore, Md). 2016;64(1):73-84.

24. McCullough AJ. The clinical features, diagnosis and natural history of nonalcoholic fatty liver disease. Clinics in liver disease. 2004;8(3):521-33, viii.

25. Anderson YC, Wynter LE, Treves KF, Grant CC, Stewart JM, Cave TL, et al. Prevalence of comorbidities in obese New Zealand children and adolescents at enrolment in a community-based obesity programme. J Paediatr Child Health. 2016;52(12):1099-105.

26. Goyal NP, Schwimmer JB. The Progression and Natural History of Pediatric Nonalcoholic Fatty Liver Disease. Clinics in liver disease. 2016;20(2):325-38.

27. Feldstein AE, Charatcharoenwitthaya P, Treeprasertsuk S, Benson JT, Enders FB, Angulo P. The natural history of non-alcoholic fatty liver disease in children: a follow-up study for up to 20 years. Gut. 2009;58(11):1538-44.

28. HH AK, Henderson J, Vanhoesen K, Ghishan F, Bhattacharyya A. Nonalcoholic fatty liver disease in children: a single center experience. Clinical gastroenterology and hepatology : the official clinical practice journal of the American Gastroenterological Association. 2008;6(7):799-802.

29. Prokopowicz Z, Malecka-Tendera E, Matusik P. Predictive Value of Adiposity Level, Metabolic Syndrome, and Insulin Resistance for the Risk of Nonalcoholic Fatty Liver Disease Diagnosis in Obese Children. Canadian journal of gastroenterology \& hepatology. 2018;2018:9465784.

30. Jinjuvadia R, Antaki F, Lohia P, Liangpunsakul S. The Association Between Nonalcoholic Fatty Liver Disease and Metabolic Abnormalities in The United States Population. Journal of clinical gastroenterology. 2017;51(2):160-6.

31. Oikonomou D, Georgiopoulos G, Katsi V, Kourek C, Tsioufis C, Alexopoulou A, et al. Nonalcoholic fatty liver disease and hypertension: coprevalent or correlated? European journal of gastroenterology \& hepatology. 2018;30(9):979-85.

32. Dai $X$, Wang B. Role of gut barrier function in the pathogenesis of nonalcoholic Fatty liver disease. Gastroenterology research and practice. 2015;2015:287348.

33. Nati M, Haddad D, Birkenfeld AL, Koch CA, Chavakis T, Chatzigeorgiou A. The role of immune cells in metabolism-related liver inflammation and development of non-alcoholic steatohepatitis (NASH). Reviews in endocrine \& metabolic disorders. 2016;17(1):29-39.

34. Mann JP, Raponi M, Nobili V. Clinical implications of understanding the association between oxidative stress and pediatric NAFLD. Expert review of gastroenterology \& hepatology. 2017;11(4):371-82. 
35. Ashraf NU, Sheikh TA. Endoplasmic reticulum stress and Oxidative stress in the pathogenesis of Non-alcoholic fatty liver disease. Free radical research. 2015;49(12):140518.

36. Chen LZ, Xia HH, Xin YN, Lin ZH, Xuan SY. TM6SF2 E167K Variant, a Novel Genetic Susceptibility Variant, Contributing to Nonalcoholic Fatty Liver Disease. Journal of clinical and translational hepatology. 2015;3(4):265-70.

37. Romeo S, Kozlitina J, Xing C, Pertsemlidis A, Cox D, Pennacchio LA, et al. Genetic variation in PNPLA3 confers susceptibility to nonalcoholic fatty liver disease. Nature genetics. 2008;40(12):1461-5.

38. Loomba R, Schork N, Chen CH, Bettencourt R, Bhatt A, Ang B, et al. Heritability of Hepatic Fibrosis and Steatosis Based on a Prospective Twin Study. Gastroenterology. 2015;149(7):1784-93.

39. Suomela E, Oikonen M, Pitkanen N, Ahola-Olli A, Virtanen J, Parkkola R, et al. Childhood predictors of adult fatty liver. The Cardiovascular Risk in Young Finns Study. Journal of hepatology. 2016;65(4):784-90.

40. Kleiner DE, Makhlouf HR. Histology of Nonalcoholic Fatty Liver Disease and Nonalcoholic Steatohepatitis in Adults and Children. Clinics in liver disease. 2016;20(2):293-312.

41. White DL, Kanwal F, El-Serag HB. Association between nonalcoholic fatty liver disease and risk for hepatocellular cancer, based on systematic review. Clinical gastroenterology and hepatology : the official clinical practice journal of the American Gastroenterological Association. 2012;10(12):1342-59.e2.

42. Valenti L, Bugianesi E, Pajvani U, Targher G. Nonalcoholic fatty liver disease: cause or consequence of type 2 diabetes? Liver international : official journal of the International Association for the Study of the Liver. 2016;36(11):1563-79.

43. Sookoian S, Pirola CJ. Non-alcoholic fatty liver disease is strongly associated with carotid atherosclerosis: a systematic review. Journal of hepatology. 2008;49(4):600-7.

44. Liyanagedera S, Williams RP, Veraldi S, Nobili V, Mann JP. The pharmacological management of NAFLD in children and adolescents. Expert review of clinical pharmacology. 2017;10(11):1225-37.

45. Mann JP, Tang GY, Nobili V, Armstrong MJ. Evaluations of Lifestyle, Dietary, and Pharmacologic Treatments for Pediatric Nonalcoholic Fatty Liver Disease: A Systematic Review. Clinical gastroenterology and hepatology : the official clinical practice journal of the American Gastroenterological Association. 2019;17(8):1457-76.e7.

46. Vos MB, Abrams SH, Barlow SE, Caprio S, Daniels SR, Kohli R, et al. NASPGHAN Clinical Practice Guideline for the Diagnosis and Treatment of Nonalcoholic Fatty Liver Disease in Children: Recommendations from the Expert Committee on NAFLD (ECON) and the North American Society of Pediatric Gastroenterology, Hepatology and Nutrition (NASPGHAN). Journal of pediatric gastroenterology and nutrition. 2017;64(2):319-34.

47. Chalasani N, Younossi Z, Lavine JE, Charlton M, Cusi K, Rinella M, et al. The diagnosis and management of nonalcoholic fatty liver disease: Practice guidance from the American Association for the Study of Liver Diseases. Hepatology (Baltimore, Md). 2018;67(1):32857.

48. Utz-Melere M, Targa-Ferreira C, Lessa-Horta B, Epifanio M, Mouzaki M, Mattos AA. NonAlcoholic Fatty Liver Disease in Children and Adolescents: Lifestyle Change - a Systematic Review and Meta-Analysis. Annals of hepatology. 2018;17(3):345-54.

49. Lassailly G, Caiazzo R, Buob D, Pigeyre M, Verkindt H, Labreuche J, et al. Bariatric Surgery Reduces Features of Nonalcoholic Steatohepatitis in Morbidly Obese Patients. Gastroenterology. 2015;149(2):379-88; quiz e15-6. 
50. Dasarathy S, Dasarathy J, Khiyami A, Joseph R, Lopez R, McCullough AJ. Validity of real time ultrasound in the diagnosis of hepatic steatosis: a prospective study. Journal of hepatology. 2009;51(6):1061-7.

51. Webb M, Yeshua H, Zelber-Sagi S, Santo E, Brazowski E, Halpern Z, et al. Diagnostic value of a computerized hepatorenal index for sonographic quantification of liver steatosis. AJR American journal of roentgenology. 2009;192(4):909-14.

52. Chauhan A, Sultan LR, Furth EE, Jones LP, Khungar V, Sehgal CM. Diagnostic accuracy of hepatorenal index in the detection and grading of hepatic steatosis. Journal of clinical ultrasound : JCU. 2016;44(9):580-6.

53. Zhang B, Ding F, Chen T, Xia LH, Qian J, Lv GY. Ultrasound hepatic/renal ratio and hepatic attenuation rate for quantifying liver fat content. World journal of gastroenterology. 2014;20(47):17985-92.

54. Senior JR. Alanine aminotransferase: a clinical and regulatory tool for detecting liver injurypast, present, and future. Clinical pharmacology and therapeutics. 2012;92(3):332-9.

55. Green RM, Flamm S. AGA technical review on the evaluation of liver chemistry tests. Gastroenterology. 2002;123(4):1367-84.

56. Knop C, Singer V, Uysal Y, Schaefer A, Wolters B, Reinehr T. Extremely obese children respond better than extremely obese adolescents to lifestyle interventions. Pediatr Obes. 2015;10(1):7-14.

57. Wiegand S, Keller KM, Lob-Corzilius T, Pott W, Reinehr T, Robl M, et al. Predicting weight loss and maintenance in overweight/obese pediatric patients. Horm Res Paediatr. 2014;82(6):380-7.

58. Reinehr T, Lass N, Toschke C, Rothermel J, Lanzinger S, Holl RW. Which Amount of BMI-SDS Reduction Is Necessary to Improve Cardiovascular Risk Factors in Overweight Children? The Journal of clinical endocrinology and metabolism. 2016;101(8):3171-9.

59. Ho M, Garnett SP, Baur L, Burrows T, Stewart L, Neve M, et al. Effectiveness of lifestyle interventions in child obesity: systematic review with meta-analysis. Pediatrics. 2012;130(6):e1647-71. 


\section{Chapter 2}

Comorbidities in primary versus secondary school children with obesity and responsiveness to lifestyle intervention

Kylie Karnebeek Supriya Thapar Maartje Willeboordse Onno C.P. van Schayck Anita C.E. Vreugdenhil

The Journal of Clinical Endocrinology \& Metabolism 2019; 104: 3803-3811 


\section{Abstract}

\section{Context}

Childhood obesity increases the risk of diseases as diabetes, cardiovascular disease and non-alcoholic fatty liver disease.

\section{Objective}

To evaluate the prevalence of comorbidities in school-age children with obesity and to compare its prevalence and the effect of a lifestyle intervention between children in primary and secondary school and between boys and girls.

\section{Setting}

Centre for Overweight Adolescent and Children's Healthcare.

\section{Patients}

Comorbidities were evaluated in 149 primary and 150 secondary school children with (morbid) obesity (162 girls). The effect of lifestyle intervention was studied in 82 primary and 75 secondary school children.

\section{Intervention}

One-year interdisciplinary lifestyle intervention

\section{Results}

Insulin resistance (37\%), impaired glucose tolerance (IGT) (3\%), dyslipidemia (48\%), hypertension (7\%) and elevated liver transaminase levels (54\%) were already common in primary school children. Glomerular hyperfiltration and insulin resistance were more prevalent in secondary school children. IGT was more prevalent in girls.

The change in BMI z-score after intervention was greater in primary school children (primary vs. secondary: $-0.25 \pm 0.32$ vs. $-0.11 \pm 0.47$ ), even as the change in LDL cholesterol concentrations (primary vs. secondary: $-0.30(-0.70 ; 0.10)$ vs. -0.10 $(-0.40 ; 0.30))$ and systolic blood pressure $z$-score (primary vs. secondary: $-0.32 \pm 1.27$ vs. $0.24 \pm 1.3$ ). The change in BMI z-score, but not in comorbidities, was greater in boys (boys vs. girls: $-0.33 \pm 0.45$ vs. $-0.05 \pm 0.31$ )

\section{Conclusions}

The presence of comorbidities is already evident in primary school children with obesity. The effect of a lifestyle intervention on these comorbidities is greater in primary compared to secondary school children, stressing the need for early interventions. 


\section{Introduction}

The global prevalence of obesity in children has increased during the past decades (1), making it an important cause of morbidity and mortality around the world. In 2015, it was estimated that approximately 108 million children were obese worldwide (1). In Western Europe, the prevalence of obesity in boys and girls younger than 20 years is $7.2 \%$ and $6.4 \%$ respectively (2). In the Netherlands, the prevalence of obesity in children aged 4 to 17 years is $2.3 \%$ in boys and $3.3 \%$ in girls (3).

Obesity during childhood is an important risk factor for the development of various comorbidities including dyslipidemia, hypertension, diabetes and sleep apnea, but also non-alcoholic fatty liver disease (NAFLD) and kidney disease (4-7). Additionally, children with obesity are likely to grow up to become adults with overweight or obesity (8). Lobstein et al. have estimated that in 2025, unless we can have a larger impact on childhood obesity in the years until then, there will be around 91 million children with obesity worldwide, of which half will experience one or more comorbidities, like type 2 diabetes, hypertension or fatty liver disease (9). In adults, obesity has also been associated with an increased all-cause mortality (10). In addition to the effects of obesity on health, obesity has also been associated with decreased quality of life (11), lower work productivity (12) and an increase in utilization of healthcare services and higher healthcare costs (13), thereby not only having an impact on the life of the individual with obesity, but also on society. Altogether, these effects of the obesity epidemic stress the need for early intervention to decrease the effect on the health parameters, but also economical outcomes.

Previous studies have shown that the development of these lifestyle-related comorbidities already starts in young children $(4,14)$. Also, studies have shown that lifestyle interventions are more effective in reducing BMI z-score in younger children with extreme obesity than in adolescents with extreme obesity (15) and that younger patients were more likely to maintain weight loss (16). However, most studies examining the effect of lifestyle interventions in children, focus on the effect on BMI (z-score), rather than on the effect of the intervention on health parameters and comparisons of intervention effects between different age subgroups are scarcely made. Additionally, previous studies have shown that physical activity and sedentary behavior (17) and dietary composition (18) differ between boys and girls and that boys and girls may respond differently to physical activity interventions (19). However, knowledge regarding sex differences in the effect of combined lifestyle interventions is limited. 
Therefore, the aim of this study is to describe the prevalence of an elaborate panel of early lifestyle-related comorbidities, including parameters of metabolic and cardiovascular health, but also liver and kidney health, in a group of school-aged children with obesity, and to compare the prevalence of these comorbidities and the effect of one year of interdisciplinary lifestyle intervention between children in primary and secondary school, and between boys and girls.

\section{Methods}

\section{Participants and setting}

This study was designed and conducted within the setting of the Centre for Overweight Adolescent and Children's Healthcare at the Maastricht University Medical Centre (MUMC+). School-aged children (6-16 years; primary school 6-11 years; secondary school 12-16 years) with obesity and morbid obesity were referred to our centre by youth health services (i.e. school doctors), general practitioners and pediatricians, who have a key role in the recognition of obesity in the children in our region. Their approach to the care for obese children is documented in guidelines. In our centre children and their families are evaluated, monitored and guided as described in detail previously (20). In summary, all children underwent a comprehensive assessment before the start of the intervention to exclude underlying syndromic or endocrine conditions of obesity, to evaluate complications and risk factors, and to gain insight in behavior and (family) functioning. A follow-up assessment including all the examinations performed during the initial assessment was offered to all children after approximately one year of lifestyle intervention. The information gathered in the assessment was used to develop a care plan that was tailored to the needs of each family. All children and their families were offered individual guidance focusing on lifestyle improvements with regards to nutrition, food habits, physical activity, sleep and psychological and social aspects. Points of improvement that were identified during the initial assessment were used as focus points for making small, step-by-step lifestyle changes. These focus points were adapted throughout the intervention, depending on for instance the successfulness of making changes or the identification of new points of improvements. Additional support was provided, if for instance limited pedagogical skills or financial or psychological problems were identified as barriers for lifestyle improvement. In general, the visits to the outpatient clinic started on a monthly basis, but frequency was adjusted based on personal needs (i.e. frequency was reduced in case of weight loss and maintenance, or outpatient visits were for instance partially replaced with consultation via telephone in case of transport problems). 
In addition to the individual outpatient clinic visits, group activities related to nutrition and physical activity were organized several times a year, which children and/or parents could attend on a voluntary basis. Children, that were not already participating in regular sport activities, were encouraged to participate in a weekly one-hour physical activity group lesson (in addition to encouragement to increase physical activity levels at home). If possible, different elements of the intervention, like sports activities, were located in the patient's own neighborhood.

All school-aged children with obesity and morbid obesity that were guided in our centre were included in this study. To prevent selection bias, no children referred by other hospitals were included.

Due to the continuous inflow of new participants into the intervention program, only a part of the children had already completed one year of lifestyle intervention at time of data analysis. Only children that had completed at least one year of lifestyle intervention were included in analyses of the intervention effects.

This study was conducted according to the guidelines of the Declaration of Helsinki and was approved by the medical ethical committee of the MUMC+. Consent was obtained by the parents and/or the child. The study is registered at ClinicalTrial.gov (registration number: NCT02091544).

\section{Anthropometric measurements}

Anthropometric measurements were performed while children were barefoot and wearing only underwear. Weight was measured on a digital scale (Seca). Height was measured using a wall-mounted digital stadiometer (De Grood Metaaltechniek). Body Mass Index (BMI) was calculated and BMI z-scores were obtained using a growth analyser (Growth Analyser VE). Children were classified as obese or morbidly obese according to International Obesity Task Force (IOTF) criteria (21). In short, age- and sex-dependent cutoff points were used to categorize children as being obese (comparable to a $\mathrm{BMI}$ of 30 $\mathrm{kg} / \mathrm{m}^{2}$ in adults) or morbidly obese (comparable to a BMI of $35 \mathrm{~kg} / \mathrm{m}^{2}$ in adults). Waist circumference was measured with a non-elastic measuring tape, at the midpoint between the lower margin of the last palpable rib and the top of the iliac crest, at the end of a normal expiration. Waist circumference z-scores were determined according to reference values for Dutch children (22). All anthropometric measurements were measured once by trained health care personnel. 


\section{Glucose metabolism}

Fasting blood glucose concentrations were determined using the Cobas 8000 modular analyzer (Roche). Fasting serum insulin levels were determined with the Immulite 1000 (Siemens). After obtaining the fasting blood sample an oral glucose tolerance test (OGTT) was performed. For this test, 1.75 grams of glucose per kilogram of bodyweight was dissolved into $200 \mathrm{~mL}$ water with a maximum of 75 grams of glucose. After patients drank the glucose solution, plasma blood glucose concentrations were measured every thirty minutes during two hours. Insulin resistance was evaluated by calculating the homeostasis model assessment for insulin resistance (HOMA-IR) (HOMA-IR = fasting glucose ( $\mathrm{mmol} / \mathrm{L}) \mathrm{x}$ fasting insulin $(\mu \mathrm{U} / \mathrm{L}) / 22.5)$. HOMA-IR values higher than age- and sex-specific $75^{\text {th }}$ percentiles for children with overweight or obesity were considered abnormal, based on a study by Shashaj et al. that showed that these cut-off points are most accurate to identify children in which the HOMA-IR value can be considered 'non-physiological' and is suspected to occur alongside other aberrant cardiometabolic risk factors (23). Impaired fasting glucose (IFG) was defined as a fasting glucose concentration $\geq 5.6 \mathrm{mmol} / \mathrm{L}$, impaired glucose tolerance (IGT) as a two-hour glucose concentration $\geq 7.8$ and $<11.1$ $\mathrm{mmol} / \mathrm{L}$ and type II diabetes as a two-hour glucose concentration $\geq 11.1 \mathrm{mmol} / \mathrm{L}$ (24).

\section{Cardiovascular risk parameters}

Fasting serum total cholesterol, $\mathrm{HDL}$ cholesterol, triglyceride concentrations were determined using the Cobas 8000 modular analyzer (Roche). LDL cholesterol concentrations were calculated with the Friedewald equation (25). Dyslipidemia was defined as elevated LDL cholesterol, low HDL cholesterol and/or elevated triglyceride concentrations according to cut-off points for children (26). Ambulatory daytime blood pressure was measured approximately 20 times with an interval of 3 minutes between measurements using the Mobil-O-Graph (I.E.M. GmbH). Mean blood pressure was calculated. Systolic and diastolic blood pressure z-scores were calculated according to reference values for height and sex (27). Hypertension was defined as a systolic and/or diastolic blood pressure z-score $>2$. The presence of the metabolic syndrome was determined according to the age-based criteria from the International Diabetes Federation (28). 


\section{Parameters reflecting liver and kidney health}

Alanine transaminase (ALT) levels and creatinine concentrations were determined with the Cobas 8000 modular analyzer (Roche). For analysis in this study, the upper limit of normal for ALT was considered $22.1 \mathrm{U} / \mathrm{L}$ for girls and $25.8 \mathrm{U} / \mathrm{L}$ for boys, based on a previous study examining the healthy range of ALT concentrations in children and adolescents (29).

Estimated glomerular filtration rate was calculated according to the Schwartz formula $\left(\right.$ eGFR $\left(\mathrm{ml} / \mathrm{min} / 1.73 \mathrm{~m}^{2}\right)=36.5 \times$ (height $(\mathrm{cm}) /$ plasma creatinine $(\mu \mathrm{mol} / \mathrm{L})$ ) (30). In this formula, the eGFR is indexed for a standardized body surface area (BSA) of $1.73 \mathrm{~m}^{2}$, which is suggested to be inaccurate in children, especially in children with obesity and for longitudinal measurements (31). Therefore, the eGFR was de-indexed by multiplying the eGFR according to the Schwarz formula by the estimated BSA and dividing by 1.73 (31, 32). The BSA was estimated using the Haycock formula (BSA $=0.024265 \times$ Weight $(\mathrm{kg})^{0.5378}$ $x$ Height $(\mathrm{cm})^{0.3964}$ ) (33). Glomerular hyperfiltration was defined as a de-indexed eGFR above $135 \mathrm{ml} / \mathrm{min}(34)$.

\section{Statistical analysis}

Statistical analysis was performed with IBM SPSS Statistics 22.0. All data was tested for normality with the Shapiro-Wilk test and reported as mean \pm SD or median (interquartile range), depending on the distribution. Differences in the prevalence of early lifestylerelated aberrations between primary and secondary school children were analyzed with the Chi-square test. Differences in parameters between age categories and sex were tested with the independent samples T-test or Mann-Whitney $U$ test, as appropriate.

Regression analyses were performed for the delta (difference between pre- and posttreatment) outcomes as dependent variables, with school age category and sex as independent variables and corrected for BMI z-score at baseline and number of consultations during the intervention period. Correction for the number of consultations was performed, because previous studies have shown that the effect of lifestyle interventions on weight might be influenced by the number of consultations during the intervention period (35). In these models, an interaction term for school age category * sex was added, however did not contribute significantly in any model and was therefore left out of the final models. Corrected regression coefficients shown are unstandardized beta's for the contribution of school age category (primary school as reference) or sex (girls as reference) to the difference between pre- and post-treatment outcomes. 


\section{Results}

\section{Baseline characteristics}

Two hundred and ninety-nine children (176 obese, 123 morbidly obese; $46 \%$ boys) with a mean BMI z-score of $3.52 \pm 0.59$ and median age of $12.1(10.1 ; 14.4)$ years were included in this study. Of these children 149 were primary school aged and 150 were secondary school aged. At baseline, the boys were slightly, however not significantly, younger than the girls (respectively $11.5(10.1 ; 13.7)$ years compared to $12.4(9.9 ; 15.2)$ years; $p=0.07)$, despite having a significantly higher BMI z-score (respectively 3.57 (3.20;4.07) vs. 3.28 $(2.97 ; 3.70))$. Additional baseline characteristics and differences between subgroups (primary vs. secondary school and boys vs. girls) are presented in table 1.

Insulin resistance (37\%), impaired glucose tolerance (3\%), dyslipidemia (48\%), hypertension (7\%) and elevated liver transaminase levels (54\%) were already present in primary school children (table 2 ). Insulin resistance (52\%) and glomerular hyperfiltration (42\%) were more prevalent in secondary school children as compared to primary school children (table 2). In girls, impaired glucose tolerance was more prevalent than in boys (respectively, $6 \%$ vs. $1 \%$ ) (table 2 ). 
Table 1. Baseline characteristics, stratified for age and sex subgroups

\begin{tabular}{|c|c|c|c|c|c|}
\hline & Total group & $\begin{array}{c}\text { Primary } \\
\text { school }\end{array}$ & $\begin{array}{c}\text { Secondary } \\
\text { school }\end{array}$ & Girls & Boys \\
\hline & $N=299$ & $\mathrm{~N}=149$ & $\mathrm{~N}=150$ & $\mathrm{~N}=162$ & $N=137$ \\
\hline Age, years & $12.1(10.1 ; 14.4)$ & $10.1(8.5 ; 11.2)$ & $14.4(13.3 ; 15.9)^{\mathrm{A}}$ & $12.4(9.9 ; 15.2)$ & $11.5(10.1 ; 13.7)$ \\
\hline Sex, $M / F$ & $137 / 162$ & $76 / 73$ & $61 / 89$ & - & - \\
\hline BMI z-score & $3.52 \pm 0.59$ & $3.56 \pm 0.67$ & $3.47 \pm 0.50$ & $3.28(2.97 ; 3.70)$ & $3.57(3.20 ; 4.07)^{\mathrm{A}}$ \\
\hline Waist circumference z-score & $5.78(4.47 ; 7.24)$ & $5.01(3.89 ; 6.20)$ & $6.59(5.32 ; 7.95)^{A}$ & $5.80(4.62 ; 7.29)$ & $5.55(4.41 ; 7.16)$ \\
\hline Obese/morbidly obese & $176 / 123$ & $89 / 60$ & $87 / 63$ & $93 / 69$ & $83 / 54$ \\
\hline Total cholesterol, $\mathrm{mmol} / \mathrm{L}$ & $4.30(3.80 ; 4.90)$ & $4.30(3.80 ; 5.00)$ & $4.30(3.70 ; 4.90)$ & $4.40(3.85 ; 5.00)$ & $4.20(3.70 ; 4.90)$ \\
\hline LDL cholesterol, $\mathrm{mmol} / \mathrm{L}$ & $2.60(2.10 ; 3.10)$ & $2.55(2.10 ; 3.10)$ & $2.60(2.10 ; 3.10)$ & $2.68 \pm 0.75$ & $2.53 \pm 0.74$ \\
\hline HDL cholesterol, $\mathrm{mmol} / \mathrm{L}$ & $1.20(1.00 ; 1.40)$ & $1.20(1.00 ; 1.50)$ & $1.20(1.00 ; 1.40)$ & $1.20(1.00 ; 1.40)$ & $1.30(1.00 ; 1.50)^{A}$ \\
\hline $\begin{array}{l}\text { Triglycerides, } \mathrm{mmol} / \mathrm{L} \\
\text { Fasting glucose, } \mathrm{mmol} / \mathrm{L}\end{array}$ & $\begin{array}{l}1.01(0.73 ; 1.40) \\
4.20(3.90 ; 4.60)\end{array}$ & $\begin{array}{l}0.98(0.71 ; 1.37) \\
4.25(3.90 ; 4.60)\end{array}$ & $\begin{array}{l}1.02(0.73 ; 1.44) \\
4.20(3.90 ; 4.60)\end{array}$ & $\begin{array}{l}1.06(0.74 ; 1.52) \\
4.20(3.90 ; 4,60)\end{array}$ & $\begin{array}{l}0.97(0.71 ; 1.30) \\
4.30(4.00 ; 4.60)\end{array}$ \\
\hline HOMA-IR & $3.14(2.04 ; 4.78)$ & $2.43(1.51 ; 3.60)$ & $3.99(2.87 ; 5.48)^{\mathrm{A}}$ & $3.51(2.43 ; 5.07)$ & $2.82(1.76 ; 4.49)^{\mathrm{A}}$ \\
\hline Systolic blood pressure z-score & $0.20(-0.40 ; 0.90)$ & $0.10(-0.45 ; 0.90)$ & $0.30(-0.43 ; 0.93)$ & $0.30(-0.33 ; 1.10)$ & $0.10(0.50 ; 0.80)$ \\
\hline Diastolic blood pressure z-score & $-0.70(-1.30 ; 0.10)$ & $-0.70(-1.55 ;-0.20)$ & $-0.50(-1.20 ; 0.30)^{\mathrm{A}}$ & $-0.60(-1.20 ; 0.20)$ & $-0.80(-1.45 ;-0.05)$ \\
\hline$A L T, U / L$ & $23.5(18.0 ; 32.0)$ & $24.0(19.0 ; 31.5)$ & $23.0(17.0 ; 34.0)$ & $22.0(16.0 ; 28.0)$ & $26.5(20.0 ; 39.0)^{A}$ \\
\hline $\begin{array}{l}\text { Estimated glomerular filtration } \\
\text { rate, } \mathrm{ml} / \mathrm{min}\end{array}$ & $121.8 \pm 27.5$ & $111.4 \pm 27.0$ & $131.8 \pm 24.2^{A}$ & $122.8 \pm 29.1$ & $120.5 \pm 25.5$ \\
\hline
\end{tabular}

Data is presented as means \pm SD or median (interquartile range); $A=$ Significant difference between between primary and secondary school aged children or between boys and girls.

Table 2. Prevalence of comorbidities in primary and secondary school-age children

\begin{tabular}{lccccc}
\hline & $\begin{array}{c}\text { All } \\
\text { participants }\end{array}$ & $\begin{array}{c}\text { Primary } \\
\text { school } \\
\text { children } \\
\text { (6-11 years) }\end{array}$ & $\begin{array}{c}\text { Secondary } \\
\text { school } \\
\text { children } \\
(12-16 \text { years) }\end{array}$ & Girls & Boys \\
\hline Dyslipidemia (\%) & $\mathrm{N}=299$ & $\mathrm{~N}=149$ & $\mathrm{~N}=150$ & $\mathrm{~N}=162$ & $\mathrm{~N}=137$ \\
Increased total cholesterol (\%) & 51.9 & 48.2 & 55.4 & 56.6 & 46.2 \\
Increased LDL cholesterol (\%) & 19.1 & 22.9 & 15.5 & 17.7 & 20.8 \\
Decreased HDL cholesterol (\%) & 18.4 & 17.9 & 18.9 & 19.0 & 17.7 \\
Increased triglycerides (\%) & 31.3 & 27.9 & 34.5 & 34.2 & 27.7 \\
Insulin resistance (\%) & 24.2 & 24.1 & 24.3 & 26.6 & 21.4 \\
Impaired fasting glucose (\%) & 44,9 & 36,8 & $52,3^{\mathrm{A}}$ & 47,4 & 42,0 \\
Impaired glucose tolerance (\%) & 1.1 & 1.5 & 0.7 & 0.7 & 1.6 \\
Diabetes type II (\%) & 3.8 & 2.5 & 4.8 & 6.1 & $0.9^{\mathrm{A}}$ \\
Hypertension (\%) & 0.4 & 0.0 & 0.7 & 0.7 & 0.0 \\
Systolic hypertension (\%) & 8.7 & 7.1 & 10.3 & 11.1 & 6,0 \\
Diastolic hypertension (\%) & 8.0 & 7.1 & 9.0 & 9.8 & 6,0 \\
Metabolic syndrome (\%) & 2.4 & 0.7 & 4.1 & 3.9 & 0.8 \\
Increased ALT levels (\%) & 17.4 & 16.4 & 17.9 & 20.0 & 14.3 \\
Glomerular hyperfiltration (\%) & 50.5 & 53.6 & 47.6 & 46.8 & 55.0 \\
\hline A = significant difference in the prevalence of lifestyle-related diseases between primary and secondary school aged children or & & & & 25.4 \\
between boys and girls. & & & & &
\end{tabular}

\section{Effect of lifestyle intervention}

The effect of one year of lifestyle intervention was evaluated in 82 primary school children and 75 secondary school children. The primary school children that underwent an elaborate health screening after one year of intervention had higher total and LDL 
cholesterol concentrations compared to the primary school children that dropped out of the intervention during the first year. There were no other baseline differences between these groups. Also, there were no baseline differences between secondary school children that underwent an elaborate health screening after one year and the children that dropped out during the first year (data not shown). The drop-out rate did not differ significantly between primary and secondary school children.

After one year of lifestyle intervention there was a significant reduction in BMI z-score $(-0.18 \pm 0.40)$, total cholesterol concentrations $(-0.25 \quad(-0.63 ; 0.30))$, LDL cholesterol concentrations $(-0.10(-0.50 ; 0.20))$ and triglyceride concentrations $(-0.05$ $(-0.41 ; 0.19))$ in the total group of children (table 3$)$. In primary school children there was a significant decrease of BMI z-score $(-0.25 \pm 0.32)$, total cholesterol levels $(-0.30$ $(-0.70 ; 0,10))$, LDL cholesterol levels $(-0.30(-0.70 ; 0.10)$, systolic blood pressure $z$-score $(-0.32 \pm 1.27)$ and an increase in eGFR $(4,8 \pm 17,7)$ (table 3$)$. In secondary school children BMI z-score and eGFR were significantly reduced, respectively $-0.11 \pm 0.47$ and $-6.3 \pm$ 16.9. The effect of the intervention on BMI z-score, LDL cholesterol concentration and systolic blood pressure z-score was significantly greater in primary school children compared to secondary school children (table 3).

Regression analysis showed that school age category was a significant predictor for change in BMI z-score, LDL cholesterol concentration and systolic blood pressure z-score after intervention, also after correction for BMI z-score at baseline and the number of consultations during the intervention (table 3).

Comparison of sexes showed that the effect of the lifestyle intervention on BMI z-score was evidently greater in boys compared to girls, with a BMI z-score reduction of $-0.33 \pm$ 0.45 compared to $-0.05 \pm 0.31$. The difference in delta BMI z-score between boys and girls was still present when analysis was stratified for primary and secondary school children (data not shown). Despite the difference in the amount of weight loss, there was no sex difference for the intervention effect on the other health parameters, neither in the total group as in stratified analysis for primary and secondary school (data not shown). Regression analysis showed that sex was still a significant predictor for change in BMI zscore after correction for BMI z-score at baseline and the number of consultations during the intervention (table 3). 


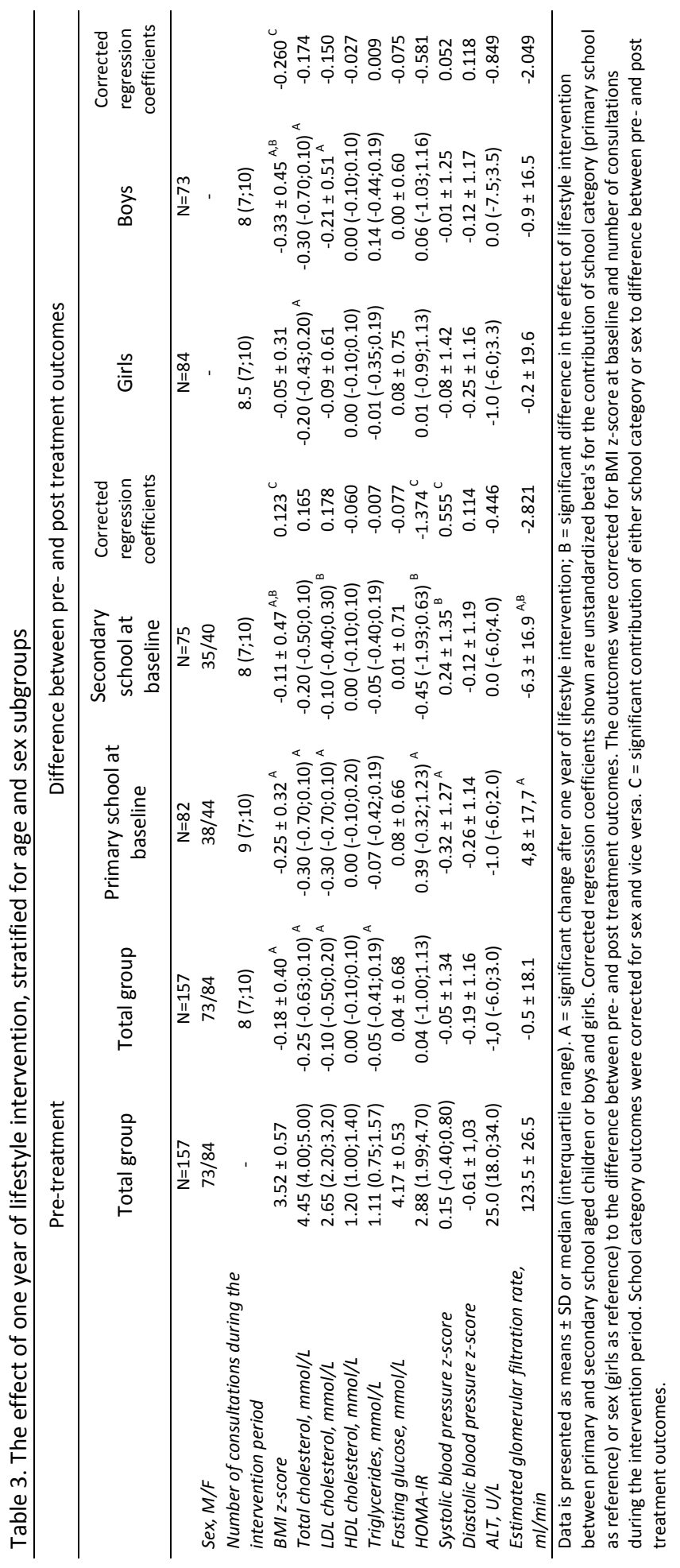




\section{Discussion}

Childhood obesity increases the risk of lifestyle-related diseases such as non-alcoholic fatty liver disease (NAFLD), diabetes, cardiovascular disease and renal disease at later age. In this study, we evaluated elaborate health parameters and the prevalence of comorbidities in primary and secondary school children with obesity and evaluated the effect of one year family-based, interdisciplinary lifestyle intervention. Remarkably, our results show that the prevalence of early lifestyle-related comorbidities hardly differs between primary school children and secondary school children with obesity and morbid obesity. Additionally, the positive effect of lifestyle intervention is greater in the younger children.

In accordance with this study, other studies from different regions across the world have also shown that these early comorbidities in children with obesity are already present from a young age. The early development of these comorbidities in our population of children with obesity and morbid obesity are comparable to the prevalence of hypertension, dyslipidemia and glucose metabolism aberrations in a large pediatric central European population that was described by Reinehr et al. (14). Notable differences between our results and the results of Reinehr et al. are a higher prevalence of decreased HDL cholesterol levels and a slightly lower prevalence of impaired glucose tolerance in our population, which for the prevalence of abnormal HDL cholesterol concentrations is most likely due to a difference in used cut-off values. I'Allemand et al. showed that elevated total cholesterol and triglyceride levels were more prevalent in children younger than 12 years old compared with children with obesity and morbid obesity aged 12-20 years, and that low HDL levels were more common in the children older than 12 years (36). In our study, we also found that the prevalence of elevated total cholesterol levels was slightly lower and the prevalence of decreased HDL cholesterol levels was slightly higher in secondary school children, however this difference did not reach statistical significance.

Remarkable differences in the results from our study compared to studies from other regions of the world mainly concerns a clear difference in glucose metabolism abnormalities. In our study, we found a low prevalence of impaired fasting glucose (1\%) and type II diabetes (0.4\%). Skinner et al. describe a prevalence of impaired fasting glucose of $23 \%$ in 1005 children with obesity (aged 12-19 years) from the United States (4). This could be explained partially by a difference in the degree of obesity between these populations and our study populations, however a direct comparison is not possible because of the different ways of reporting obesity classification. Additionally, these 
marked differences in glucose metabolism abnormalities might be (partially) due to differences in dietary habits or ethnicity $(37,38)$.

In our study, there was a significant decrease of BMI z-score after lifestyle intervention in both primary school children and secondary school children, with a greater effect in primary school children $(-0.25 \pm 0.31$ vs. $-0.11 \pm 0.47$ in secondary school children). In addition to a significant improvement of BMI z-score, we found an improvement of LDL cholesterol concentrations and systolic blood pressure z-score in primary school children, which were all significantly greater compared to secondary school children.

Previous studies have also reported a greater effect of lifestyle intervention on BMI zscore in younger children $(15,16,39,40)$. Regression analysis in our study showed that school age category was a significant predictor for change in BMI z-score, regardless of $\mathrm{BMI}$ z-score at baseline and the number of consultations during the intervention. Moreover, since both primary and secondary school children in this study are from the same population and were referred to our lifestyle intervention according to the same guidelines, we do not assume that there is a selection bias that could explain the difference in the effect of the lifestyle intervention. Perhaps, a reduced influence of parents on the health behavior of adolescents may play a role in the greater effect of lifestyle interventions in younger children.

Two recent Cochrane reviews have evaluated the effects of dietary, physical activity and behavioral randomized controlled trials for the treatment of overweight and obesity in children (6-11 years) (41) and adolescents (12-17 years) (42). These reviews show a mean decrease in BMI z-score of 0.06 units in children and 0.13 units in adolescents after intervention. The design and duration of the interventions in these reviews are however quite divers, impairing a direct comparison with our study.

In our study, there was difference in the effect of lifestyle intervention on the BMI z-score between boys and girls, with a significantly greater effect in boys. It is interesting to consider why we found this difference. Perhaps, pre-existing sex differences play a role here. For instance, previous studies have shown that boys in general are more physically active than girls (17) and that boys may be better able than girls to change several physical activity aspects, like increasing activity and decreasing sedentary time (19).

Interestingly, the improvement in health parameters during the intervention did not differ significantly between boys and girls. Perhaps, since their BMI z-score stabilized, the girls did also improve their lifestyle. This improvement might have been enough to decrease comorbidities, but not enough to decrease their BMI z-score. In that case, weight stabilization in girls might be as important as weight loss in boys. 
A major strength of this study is that it was performed in a representative group of schoolage children with obesity and morbid obesity in our region. Also, in addition to anthropometric data, which is often the focus in studies evaluating the effect of lifestyle interventions, we have data on elaborate health outcomes and of the effect of lifestyle intervention on these health parameters. A limitation of this study is that little objective data regarding behavioral changes due to the lifestyle intervention, such as changes in eating and physical activity behavior, is available, thereby limiting interpretation of which specific components of the lifestyle changes have led to the health improvements we found.

\section{Conclusion}

The early development of comorbidities is already evident in primary school aged children with obesity and morbid obesity. The positive effect of lifestyle intervention on these comorbidities is greater in primary school children compared to secondary school children, reflecting the need to start intervention as early as possible, but also to have attention for healthy lifestyle already early on in primary school to prevent the development of obesity and the accompanying health effects. 


\section{References}

1. Afshin A, Forouzanfar MH, Reitsma MB, Sur P, Estep K, Lee A, et al. Health Effects of Overweight and Obesity in 195 Countries over 25 Years. The New England journal of medicine. 2017;377(1):13-27.

2. Ng M, Fleming T, Robinson M, Thomson B, Graetz N, Margono C, et al. Global, regional, and national prevalence of overweight and obesity in children and adults during 19802013: a systematic analysis for the Global Burden of Disease Study 2013. Lancet (London, England). 2014;384(9945):766-81.

3. Corte CD, Ferrari F, Villani A, Nobili V. Epidemiology and Natural History of NAFLD. Journal of medical biochemistry. 2015;34(1):13-7.

4. Skinner AC, Perrin EM, Moss LA, Skelton JA. Cardiometabolic Risks and Severity of Obesity in Children and Young Adults. The New England journal of medicine. 2015;373(14):130717.

5. Perrone J, Hollander JE, De Roos F. Cardiovascular risk factors and atherosclerosis in children and young adults. The New England journal of medicine. 1998;339(15):1083-4.

6. Corey KE, Kaplan LM. Obesity and liver disease: the epidemic of the twenty-first century. Clinics in liver disease. 2014;18(1):1-18.

7. Herouvi D, Karanasios E, Karayianni C, Karavanaki K. Cardiovascular disease in childhood: the role of obesity. Eur J Pediatr. 2013;172(6):721-32.

8. Juhola J, Magnussen CG, Viikari JS, Kahonen M, Hutri-Kahonen N, Jula A, et al. Tracking of serum lipid levels, blood pressure, and body mass index from childhood to adulthood: the Cardiovascular Risk in Young Finns Study. The Journal of pediatrics. 2011;159(4):584-90.

9. Lobstein T, Jackson-Leach R. Planning for the worst: estimates of obesity and comorbidities in school-age children in 2025. Pediatr Obes. 2016;11(5):321-5.

10. Flegal KM, Kit BK, Orpana H, Graubard BI. Association of all-cause mortality with overweight and obesity using standard body mass index categories: a systematic review and meta-analysis. JAMA. 2013;309(1):71-82.

11. Tsiros MD, Olds T, Buckley JD, Grimshaw P, Brennan L, Walkley J, et al. Health-related quality of life in obese children and adolescents. Int J Obes (Lond). 2009;33(4):387-400.

12. Hammond RA, Levine R. The economic impact of obesity in the United States. Diabetes, metabolic syndrome and obesity : targets and therapy. 2010;3:285-95.

13. Kamble PS, Hayden J, Collins J, Harvey RA, Suehs B, Renda A, et al. Association of obesity with healthcare resource utilization and costs in a commercial population. Current medical research and opinion. 2018:1-18.

14. Reinehr T, Wiegand S, Siegfried W, Keller KM, Widhalm K, I'Allemand D, et al. Comorbidities in overweight children and adolescents: do we treat them effectively? Int J Obes (Lond). 2013;37(4):493-9.

15. Knop C, Singer V, Uysal Y, Schaefer A, Wolters B, Reinehr T. Extremely obese children respond better than extremely obese adolescents to lifestyle interventions. Pediatr Obes. 2015;10(1):7-14.

16. Wiegand S, Keller KM, Lob-Corzilius T, Pott W, Reinehr T, Robl M, et al. Predicting weight loss and maintenance in overweight/obese pediatric patients. Horm Res Paediatr. 2014;82(6):380-7.

17. Chinapaw MJ, Yildirim M, Altenburg TM, Singh AS, Kovacs E, Molnar D, et al. Objective and self-rated sedentary time and indicators of metabolic health in Dutch and Hungarian 10-12 year olds: the ENERGY-Project. PloS one. 2012;7(5):e36657. 
18. Hiza HA, Casavale KO, Guenther PM, Davis CA. Diet quality of Americans differs by age, sex, race/ethnicity, income, and education level. Journal of the Academy of Nutrition and Dietetics. 2013;113(2):297-306.

19. Epstein LH, Paluch RA, Raynor HA. Sex differences in obese children and siblings in familybased obesity treatment. Obes Res. 2001;9(12):746-53.

20. Rijks JM, Plat J, Mensink RP, Dorenbos E, Buurman WA, Vreugdenhil AC. Children With Morbid Obesity Benefit Equally as Children With Overweight and Obesity From an Ongoing Care Program. The Journal of clinical endocrinology and metabolism. 2015;100(9):3572-80.

21. Cole TJ, Lobstein T. Extended international (IOTF) body mass index cut-offs for thinness, overweight and obesity. Pediatr Obes. 2012;7(4):284-94.

22. Fredriks AM, van Buuren S, Fekkes M, Verloove-Vanhorick SP, Wit JM. Are age references for waist circumference, hip circumference and waist-hip ratio in Dutch children useful in clinical practice? Eur J Pediatr. 2005;164(4):216-22.

23. Shashaj B, Luciano R, Contoli B, Morino GS, Spreghini MR, Rustico C, et al. Reference ranges of HOMA-IR in normal-weight and obese young Caucasians. Acta diabetologica. 2016;53(2):251-60.

24. Standards of medical care in diabetes--2014. Diabetes care. 2014;37 Suppl 1:S14-80.

25. Friedewald WT, Levy RI, Fredrickson DS. Estimation of the concentration of low-density lipoprotein cholesterol in plasma, without use of the preparative ultracentrifuge. Clin Chem. 1972;18(6):499-502.

26. Expert panel on integrated guidelines for cardiovascular health and risk reduction in children and adolescents: summary report. Pediatrics. 2011;128 Suppl 5:S213-56.

27. Wuhl E, Witte K, Soergel M, Mehls O, Schaefer F. Distribution of 24-h ambulatory blood pressure in children: normalized reference values and role of body dimensions. J Hypertens. 2002;20(10):1995-2007.

28. Zimmet P, Alberti KG, Kaufman F, Tajima N, Silink M, Arslanian S, et al. The metabolic syndrome in children and adolescents - an IDF consensus report. Pediatr Diabetes. 2007;8(5):299-306.

29. Schwimmer JB, Dunn W, Norman GJ, Pardee PE, Middleton MS, Kerkar N, et al. SAFETY study: alanine aminotransferase cutoff values are set too high for reliable detection of pediatric chronic liver disease. Gastroenterology. 2010;138(4):1357-64, 64 e1-2.

30. Schwartz GJ, Munoz A, Schneider MF, Mak RH, Kaskel F, Warady BA, et al. New equations to estimate GFR in children with CKD. J Am Soc Nephrol. 2009;20(3):629-37.

31. Pottel H. Measuring and estimating glomerular filtration rate in children. Pediatr Nephrol. 2017;32(2):249-63.

32. Correia-Costa L, Schaefer F, Afonso AC, Bustorff M, Guimaraes JT, Guerra A, et al. Normalization of glomerular filtration rate in obese children. Pediatric nephrology (Berlin, Germany). 2016;31(8):1321-8.

33. Haycock GB, Schwartz GJ, Wisotsky DH. Geometric method for measuring body surface area: a height-weight formula validated in infants, children, and adults. J Pediatr. 1978;93(1):62-6.

34. Piepsz A, Tondeur M, Ham H. Revisiting normal (51)Cr-ethylenediaminetetraacetic acid clearance values in children. Eur J Nucl Med Mol Imaging. 2006;33(12):1477-82.

35. Janicke DM, Steele RG, Gayes LA, Lim CS, Clifford LM, Schneider EM, et al. Systematic review and meta-analysis of comprehensive behavioral family lifestyle interventions addressing pediatric obesity. Journal of pediatric psychology. 2014;39(8):809-25.

36. I'Allemand D, Wiegand S, Reinehr T, Muller J, Wabitsch M, Widhalm K, et al. Cardiovascular risk in 26,008 European overweight children as established by a multicenter database. Obesity (Silver Spring). 2008;16(7):1672-9. 
37. Razak F, Anand S, Vuksan V, Davis B, Jacobs R, Teo KK, et al. Ethnic differences in the relationships between obesity and glucose-metabolic abnormalities: a cross-sectional population-based study. Int J Obes (Lond). 2005;29(6):656-67.

38. Hu FB. Globalization of diabetes: the role of diet, lifestyle, and genes. Diabetes care. 2011;34(6):1249-57.

39. Reinehr T, Kleber M, Lass N, Toschke AM. Body mass index patterns over $5 \mathrm{y}$ in obese children motivated to participate in a 1-y lifestyle intervention: age as a predictor of longterm success. Am J Clin Nutr. 2010;91(5):1165-71.

40. de Niet J, Timman R, Rokx C, Jongejan M, Passchier J, van Den Akker E. Somatic complaints and social competence predict success in childhood overweight treatment. Int J Pediatr Obes. 2011;6(2-2):e472-9.

41. Mead E, Brown T, Rees K, Azevedo LB, Whittaker V, Jones D, et al. Diet, physical activity and behavioural interventions for the treatment of overweight or obese children from the age of 6 to 11 years. The Cochrane database of systematic reviews. 2017;6:Cd012651.

42. Al-Khudairy L, Loveman E, Colquitt JL, Mead E, Johnson RE, Fraser H, et al. Diet, physical activity and behavioural interventions for the treatment of overweight or obese adolescents aged 12 to 17 years. The Cochrane database of systematic reviews. 2017;6:Cd012691. 
Chapter 2 


\section{Chapter 3}

Changes in free-living glycemic profiles after 12 months of lifestyle intervention in children with overweight and obesity

Kylie Karnebeek* Jesse M. Rijks*

Elke Dorenbos Willem-Jan M. Gerver Jogchum Plat Anita C.E. Vreugdenhil

* Contributed equally

Submitted 


\section{Abstract}

\section{Introduction}

Glycemic variability has been associated with endothelial dysfunction. Previous studies demonstrated that hyperglycemic glucose concentrations are frequently observed in children with overweight and obesity. The aim of this study is to evaluate the effect of 12 months lifestyle intervention on free-living glycemic profiles in children with overweight and obesity, and the association of alterations in these profiles with changes in cardiovascular risk parameters.

\section{Methods}

BMI z-score, free-living glycemic profiles, continuous overlapping net glycemic action (CONGA), and cardiovascular parameters were evaluated before and after multidisciplinary lifestyle intervention, in 33 non-diabetic children (39\% boys) with overweight and obesity.

\section{Results}

The median sensor glucose concentration was $5.0(3.2-7.3) \mathrm{mmol} / \mathrm{L}$ at baseline and did not change significantly after intervention. In the children with a decrease in BMI z-score after intervention, the duration of glucose concentrations above the high-normal threshold $(6.7 \mathrm{mmol} / \mathrm{L})$ and the glycemic variability decreased significantly. In these children, a decrease in median sensor glucose was associated with a decrease in LDLcholesterol, systolic and diastolic blood pressure z-score. A decrease in BMI z-score was associated with a decrease in CONGA1, 2, and 4.

\section{Conclusion}

Glycemic profiles in free-living conditions in children with overweight and obesity improved in children with a decrease in BMI z-score after lifestyle intervention. Changes in median sensor glucose concentrations were associated with changes in LDL-cholesterol and blood pressure z-scores, in children with a decrease in BMI z-score. These results suggest that lifestyle intervention can result in improvement of glucose homeostasis and cardiovascular health. 


\section{Introduction}

It is well acknowledged that children with overweight and (morbid) obesity are at risk for developing type 2 diabetes mellitus (T2DM) and cardiovascular diseases (CVD) $(1,2)$. There are strong suggestions that mild glycemic dysregulation, which precedes the actual onset of T2DM, contributes substantially to the development of endothelial dysfunction $(3,4)$. Several studies have shown aberrant cardiometabolic risk profiles already at a young age in children with overweight and (morbid) obesity $(5,6)$. In a recent study we demonstrated that besides the presence of an increased CVD risk, glucose homeostasis is already disturbed in these children (7). Hypoglycemic and hyperglycemic excursions (respectively in $73 \%$ and $27 \%$ of the children) were frequently observed in children with overweight and (morbid) obesity, using a continuous glucose monitor (CGM) sensor in free-living conditions (7). In that study, $91 \%$ of the glucose measurements were within the normoglycemic range $(\geq 3.9-<7.8 \mathrm{mmol} / \mathrm{L})$. Chan et al. also demonstrated hyperglycemic excursions in free-living conditions in adolescents with obesity and pre-diabetes (8). Another previous study showed that hypo- and hyperglycemia was less frequent in children with a normal weight, with hypoglycemic excursions in $24 \%$ of the children and $97 \%$ of the glucose measurements within the normoglycemic range (9). Altogether these findings suggest that the vascular system of children with overweight and (morbid) obesity is already exposed to glycemic dysregulation at an early age. This exposure is likely to be harmful, and indeed duration and magnitude of hyperglycemic glucose excursions was demonstrated to be associated with cardiovascular risk parameters such as triacylglycerol concentrations and waist circumference in children with overweight and (morbid) obesity (7). Moreover, it was shown in healthy adults and adults with T2DM that a high frequency and amplitude of glucose fluctuations during the day (high glycemic variability) initiated oxidative stress pathways and pro-inflammatory cytokine secretion, both having harmful effects on vascular function (10-13). Moreover, besides affecting the peripheral vasculature, a high glycemic variability was shown to have a negative effect on brain development in children with type 1 diabetes (14).

In adults with T2DM, glucose disturbances were shown to be reversible since lifestyle interventions improving dietary behaviour or physical activity both resulted in a significant improvement of glycemic variability in free-living conditions (15-18). Current studies investigating continuous glucose monitoring in free-living conditions in children focused mainly on the use of these measurements in individuals with diabetes (19-21). Studies investigating glycemic profiles in free-living conditions in non-diabetic children are scarce, are limited to cross-sectional evaluations and the effects of lifestyle improvement on glycemic profiles in free-living conditions are unknown $(7,8)$. Furthermore, it needs to be 
explored whether improvement of glucose homeostasis due to lifestyle changes translates in cardiovascular health benefits in children with overweight and (morbid) obesity. Therefore, the aim of the present study was to evaluate the effect of 12 months lifestyle intervention on glycemic profiles in children with overweight and (morbid) obesity in freeliving conditions, and to evaluate the association of alterations in these profiles with changes in cardiovascular risk parameters.

\section{Material and Methods}

\section{Setting}

This study was designed and conducted within the setting of the Centre for Overweight Adolescent and Children's Healthcare ( $\mathrm{COACH})$ at the Maastricht University Medical Centre (MUMC+). Within $\mathrm{COACH}$, the health status of children with overweight and (morbid) obesity and their families was evaluated, they were monitored and received lifestyle coaching as described previously (5). Briefly, partaking in the $\mathrm{COACH}$ program commenced with a comprehensive assessment aimed to exclude underlying syndromic or endocrine conditions of overweight, evaluate complications and risk factors, and obtain insight into behaviour and family functioning. The assessment included, amongst others, a CGM sensor measurement and an oral glucose tolerance test (OGTT). After the assessment, all children and their families were offered on-going, tailored and individual guidance with foci on lifestyle changes on a frequent basis at the outpatient clinic. Furthermore, participation in sports activities in groups and activities aimed at increasing nutritional knowledge were offered. A follow-up assessment including all the examinations performed during the initial assessment was offered annually to all children.

\section{Study participants}

All 43 children with complete CGM sensor data at baseline and who had additional CGM sensor measurement after 12 months intervention were considered for inclusion in this study. Children with incomplete CGM sensor data after 12 months intervention were excluded from this study $(n=10)$. Finally, 33 children were eligible for inclusion. The study was conducted according the guidelines administered by the Declaration of Helsinki and approved by the medical ethical committee of the MUMC+ and registered at ClinicalTrial.gov as NCT02091544. 


\section{Participant characteristics}

Anthropometric measurements were acquired while children were barefoot and wearing only underwear. Body weight was determined using a digital scale (Seca) and body length was measured using a digital stadiometer (De Grood Metaaltechniek). Body mass index (BMI) was calculated and BMI z-scores were obtained using a growth analyser (Growth Analyser VE) based upon reference charts of the Dutch nationwide growth study (22). Based on the International Obesity Task Force criteria children were classified as overweight, obese, or morbidly obese (23). Waist circumference was measured with a non-elastic tape at the end of a natural breath at midpoint between the top of the iliac crest and the lower margin of the last palpable rib. Waist circumference z-scores were calculated according to age references for Dutch children (24). Ethnicity was defined based on the definition of the Dutch Central Agency for Statistics (25).

\section{Glucose metabolism}

Fasting plasma glucose concentrations (Immulite-1000, Siemens Healthcare Diagnostics), serum insulin concentrations (Immulite-1000, Siemens Healthcare Diagnostics), and HbA1c concentrations (HPLC Variant II, Bio-Rad Laboratories) were determined. After obtaining the fasting blood sample an OGTT was performed. 1.75 grams of glucose per kilogram of bodyweight was dissolved into $200 \mathrm{~mL}$ water, with a maximum of 75 grams of glucose in total, and given orally. Plasma blood glucose concentrations were measured every thirty minutes during two hours. Impaired fasting glucose (IFG; fasting glucose $5.6-$ $6.9 \mathrm{mmol} / \mathrm{L}$ ), IGT (glucose $\geq 7.8$ - $<11.1 \mathrm{mmol} / \mathrm{L}$ after 2 -hours), T2DM (fasting glucose $\geq 7.0$ $\mathrm{mmol} / \mathrm{L}$ or glucose $\geq 11.1 \mathrm{mmol} / \mathrm{L}$ after 2-hours), and elevated HbA1c concentrations $(\geq 5.7 \%)$ were defined according to the American Diabetes Association (ADA) criteria (26). In this study, insulin resistance was estimated using the HOMA-IR (26). The following formula was applied: fasting glucose ( $\mathrm{mmol} / \mathrm{L}) \times$ fasting insulin $(\mu \mathrm{U} / \mathrm{L}) / 22.5$ (27). A cut-off point of 2.5 was used based on adult standards to determine the presence of insulin resistance (27).

Glucose concentrations in free-living conditions were measured using a CGM sensor (MiniMed, Metronic), as described previously (7). In short, sensor glucose concentrations were measured for 48-hours in free-living conditions and median, minimum, and maximum glucose concentrations were calculated. The duration of glucose concentrations in the hypoglycemic range (level 1: 3.9-3.0 mmol/L; level $2:<3.0 \mathrm{mmol} / \mathrm{L}$ ), the target range (3.9-10.0 mmol/L; secondary 3.9-7.8 mmol/L) and hyperglycemic range (level $1:>10.0$ $\mathrm{mmol} / \mathrm{L}$; level 2: $13.9 \mathrm{mmol} / \mathrm{L}$ ) were calculated according to the International Consensus 
on Use of Continuous Glucose Monitoring (28). Additionally, since the children that were included in this study did not have diabetes and were therefore unlikely to have glucose concentrations in the aforementioned hyperglycemic level 1 and 2 ranges, but could be expected to have high normal or mildly elevated glucose concentrations, the duration of glucose concentrations $\geq 6.7 \mathrm{mmol} / \mathrm{L}$ and $\geq 7.8 \mathrm{mmol} / \mathrm{L}(28,29)$ was also calculated. The sensor glucose AUC was calculated using the trapezoidal method. Overall glycemic variability during the whole 48 -hour period was assessed with the coefficient of variation (CV), which was calculated as: (standard deviation/mean)*100\%. The sensor glucose measurements were also stratified for daytime and nighttime glucose measurements (day: 7 AM - 10 PM; night 10 PM - 7 AM). Furthermore, the intra-day glycemic variability, which reflects acute glucose fluctuations throughout the day, was assessed by the continuous overlapping net glycemic action (CONGA). With this method, the difference between each glucose reading and the glucose reading certain hours previously is calculated (30). The CONGA is the standard deviation of the differences. In this study, CONGA1, CONGA2, and CONGA4 were used based on 1-, 2- and 4-hour time differences, respectively.

\section{Cardiovascular risk parameters}

Fasting lipid and lipoprotein profiles, including serum total cholesterol (TC), LDLcholesterol (LCL-C), HDL-cholesterol (HDL-C), and TAG concentrations, were measured (Cobas 8000 modular analyser, Roche). Daytime blood pressure (BP) was measured during a period of 1.5 hours for approximately 20 times with an interval of three minutes between each measurement using the Mobil-O-Graph (I.E.M. GmbH). Based on these 20 measurements the mean BP was calculated. The size of the cuff used corresponded with the circumference of the upper arm. Systolic- and diastolic BP z-scores were calculated according to reference values related to height and gender (31).

\section{Statistical analysis}

All statistical analyses were performed using SPSS 20.0 for Windows (SPSS Inc, Chicago, IL). BMI z-score, sensor glucose measurements, and cardiometabolic risk parameters at baseline and after 12 months lifestyle intervention were compared using the paired Student's T-test, the Wilcoxon signed-rank test, or the $\chi^{2}$ test, as appropriate. Correlations between variables were determined by Spearman's correlation analysis. Data are presented as means with standard deviations or as medians with the minimum and 
maximum. For all analysis, a p-value below 0.05 was considered to be statistically significant.

\section{Results}

\section{Participant characteristics at baseline and after 12 months intervention}

Thirty-three, predominantly Caucasian (85\%) children (13 boys, 20 girls) with a mean age of $12.5 \pm 3.2$ years old were enrolled in these analyses. At baseline, nine percent (\%) was overweight $(n=3), 42 \%(n=14)$ was obese, and $49 \%(n=16)$ morbidly obese. Despite the wide range in $\mathrm{BMI} z$-scores, all children had fasting glucose concentrations within the normal range $(<5.6 \mathrm{mmol} / \mathrm{L})$. The mean HOMA-IR was $3.31 \pm 1.61$. Based on these HOMAIR values, insulin resistance was present in $58 \%(n=19)$ of the children. Based on glucose concentrations $\geq 7.8 \mathrm{mmol} / \mathrm{L}$ two hours after the glucose load only one child was classified as having impaired glucose tolerance. $\mathrm{HbA1c}$ concentrations were elevated in $27 \%(n=9)$ of the children. After 12 months lifestyle intervention the BMI z-score did not improve significantly in the complete group $(p=0.206)$, whereas there was a significant improvement in weight status classification $(p<0.001)$, i.e. a shift from morbidly obese to obese, or obese to overweight (table 1). TC ( $p=0.018)$, LDL-C $(p=0.025)$, and HbA1c concentrations $(p<0.001)$ decreased significantly in the complete group after 12 months lifestyle intervention (table 1$)$, while insulin concentrations $(p=0.014)$ and HOMA-IR $(p=0.014)$ increased significantly. Characteristics of the children at baseline and after 12 months lifestyle intervention are presented in table 1.

Based on changes in BMI z-score after 12 months lifestyle intervention children were stratified in two groups: (1) children with a decrease in BMI z-score; (2) children with an increase in BMI z-score. Sixty-one percent $(n=20)$ of the children successfully improved their BMI z-score with a significant decrease of $-0.24 \pm 0.15$ units $(p<0.001)$. BMI z-score increased significantly with $0.21 \pm 0.17$ units $(p=0.001)$ in the remaining $39 \%(n=13)$ of the children. Children with a decrease in BMI z-score over time were significantly younger at baseline as compared with children that showed an increase in BMI z-score. There were no significant differences at baseline between these groups regarding anthropometric measurements and cardiovascular risk parameters (table 2). Significant improvements in TC and LDL-C were only demonstrated in children with a decrease in BMI z-score. HbA1C concentrations improved significantly in both groups (table 2). A significant increase in insulin concentrations and HOMA-IR were only found in the children with an increase in BMI z- score (table 2). 
Table 1. Characteristics of the study participants at baseline and after 12 months lifestyle intervention

\begin{tabular}{|c|c|c|}
\hline & Baseline & $\begin{array}{c}\text { After } 12 \text { months } \\
\text { intervention }\end{array}$ \\
\hline BMI z-score & $3.53 \pm 0.66$ & $3.46 \pm 0.67$ \\
\hline Overweight/ obesity/ morbid obesity, \% & $9 / 42 / 49$ & $15 / 39 / 46^{A}$ \\
\hline Waist circumference z-score & $6.85 \pm 2.43$ & $7.33 \pm 2.12^{\mathrm{A}}$ \\
\hline Median sensor glucose, $\mathrm{mmol} / \mathrm{L}$ & $5.0(3.2-7.3)$ & $5.1(3.6-6.9)$ \\
\hline Maximum sensor glucose, $\mathrm{mmol} / \mathrm{L}$ & $7.2(5.6-11.2)$ & $7.0(5.4-9.9)$ \\
\hline Minimum sensor glucose, $\mathrm{mmol} / \mathrm{L}$ & $3.4(2.2-4.4)$ & $3.4(2.2-4.9)$ \\
\hline Sensor glucose area under the curve & $14867 \pm 1447$ & $14746 \pm 1586$ \\
\hline CONGA1 & $0.57(0.39-1.31)$ & $0.50(0.30-1.08)^{A}$ \\
\hline CONGA2 & $0.72(0.46-1.61)$ & $0.69(0.30-1.58)$ \\
\hline CONGA4 & $0.88(0.45-2.02)$ & $0.87(0.39-1.94)$ \\
\hline$C V, \%$ & $15.7 \pm 5.5$ & $15.1 \pm 4.0$ \\
\hline Fasting glucose, $\mathrm{mmol} / \mathrm{L}$ & $4.0 \pm 0.5$ & $4.0 \pm 0.5$ \\
\hline Fasting insulin, $\mathrm{mU} / \mathrm{L}$ & $18.5 \pm 9.2$ & $25.6 \pm 13.7^{\mathrm{A}}$ \\
\hline HOMA-IR & $3.31 \pm 1.61$ & $4.29 \pm 2.30^{A}$ \\
\hline $\mathrm{HbA} 1 \mathrm{c}, \%$ & $5.4 \pm 0.3$ & $5.2 \pm 0.4^{A}$ \\
\hline Plasma glucose 2-hours after glucose load, $\mathrm{mmol} / \mathrm{L}$ & $5.5 \pm 1.2$ & $5.6 \pm 1.1$ \\
\hline Total cholesterol, $\mathrm{mmol} / \mathrm{L}$ & $4.8(3.5-6.6)$ & $4.5(3.5-6.9)^{A}$ \\
\hline LDL-cholesterol, mmol/L & $3.1(2.0-4.5)$ & $2.7(1.7-4.6)^{A}$ \\
\hline HDL-cholesterol, $\mathrm{mmol} / \mathrm{L}$ & $1.1(0.8-1.9)$ & $1.1(0.8-1.9)$ \\
\hline Triglycerides, $\mathrm{mmol} / \mathrm{L}$ & $1.21(0.39-4.48)$ & $1.13(0.51-3.77)$ \\
\hline Systolic blood pressure z-score & $0.19 \pm 1.26$ & $0.02 \pm 1.16$ \\
\hline Diastolic blood pressure z-score & $-0.37 \pm 0.88$ & $-0.66 \pm 1.17$ \\
\hline
\end{tabular}

Table 2. Characteristics of the study participants at baseline and after 12 months lifestyle intervention stratified for change in BMI z-score

\begin{tabular}{|c|c|c|c|c|}
\hline & \multicolumn{2}{|c|}{$\begin{array}{l}\text { Decrease in BMI z-score after } 12 \\
\text { months intervention } \\
\qquad(\mathrm{n}=20)\end{array}$} & \multicolumn{2}{|c|}{$\begin{array}{l}\text { Increase in BMI z-score after } 12 \\
\text { months intervention } \\
(\mathrm{n}=13)\end{array}$} \\
\hline & Baseline & $\begin{array}{l}12 \text { months } \\
\text { intervention }\end{array}$ & Baseline & $\begin{array}{l}12 \text { months } \\
\text { intervention }\end{array}$ \\
\hline BMI z-score & $3.54 \pm 0.62$ & $3.30 \pm 0.61^{\mathrm{A}}$ & $3.50 \pm 0.74$ & $3.71 \pm 0.71^{\mathrm{A}}$ \\
\hline Waist circumference z-score & $6.3 \pm 1.9$ & $6.9 \pm 1.6$ & $7.6 \pm 3.4$ & $8.2 \pm 3.1$ \\
\hline Fasting glucose, $\mathrm{mmol} / \mathrm{L}$ & $4.1 \pm 0.6$ & $4.3 \pm 0.4$ & $3.9 \pm 0.5$ & $4.1 \pm 0.6$ \\
\hline Fasting insulin, $\mathrm{mU} / \mathrm{L}$ & $18.1 \pm 7.0$ & $20.7 \pm 9.8$ & $20.2 \pm 12.4$ & $27.5 \pm 13.3^{\mathrm{A}}$ \\
\hline HOMA-IR & $3.21 \pm 1.40$ & $4.00 \pm 2.00$ & $3.20 \pm 1.84$ & $4.50 \pm 2.48$ \\
\hline $\mathrm{HbA} 1 \mathrm{c}, \%$ & $5.4 \pm 0.3$ & $5.2 \pm 0.4^{\mathrm{A}}$ & $5.5 \pm 0.2$ & $5.2 \pm 0.4^{\mathrm{A}}$ \\
\hline $\begin{array}{l}\text { Plasma glucose 2-hours after glucose } \\
\text { load, } \mathrm{mmol} / \mathrm{L}\end{array}$ & $5.6(3.9-7.5)$ & $5.6(4.2-6.7)$ & $4.8(2.9-6.3)$ & $5.2(4.2-8.5)$ \\
\hline Total cholesterol, mmol/L & $5.0 \pm 0.9$ & $4.4 \pm 0.6^{\mathrm{A}}$ & $4.9 \pm 0.7$ & $5.0 \pm 0.9$ \\
\hline LDL-cholesterol, mmol/L & $3.2 \pm 0.8$ & $2.7 \pm 0.6^{\mathrm{A}}$ & $2.9 \pm 0.7$ & $3.0 \pm 0.7$ \\
\hline HDL-cholesterol, mmol/L & $1.1 \pm 0.2$ & $1.2 \pm 0.2$ & $1.3 \pm 0.4$ & $1.2 \pm 0.3$ \\
\hline Triglycerides, mmol/L & $1.23(0.49-3.11)$ & $1.02(0.51-3.69)$ & $1.20(0.39-4.48)$ & $1.28(0.65-3.77)$ \\
\hline Systolic blood pressure z-score & $0.23 \pm 1.09$ & $-0.11 \pm 1.07$ & $0.11 \pm 1.63$ & $0.21 \pm 1.37$ \\
\hline Diastolic blood pressure z-score & $-0.55 \pm 0.65$ & $-0.89 \pm 1.23$ & $-0.17 \pm 1.15$ & $-0.16 \pm 0.95$ \\
\hline
\end{tabular}




\section{8-hour glycemic profile analysis at baseline and after 12 months intervention}

At baseline, the median sensor glucose concentration was $5.0(3.2-7.3) \mathrm{mmol} / \mathrm{L}$ and did not change significantly after 12 months lifestyle intervention ( $p=0.431$ ) (table 1$)$. CONGA1 decreased significantly after 12 months lifestyle intervention from $0.57(0.39-1.31)$ to $0.50(0.30-1.08)(p=0.048)$ (table 1$)$, but CONGA2 and CONGA4 did not change significantly ( $p=0.091 ; p=0.228$, respectively).

At baseline, sixty-four percent $(n=21)$ of the children reached high-normal sensor glucose concentrations defined as values $\geq 6.7 \mathrm{mmol} / \mathrm{L}$ for a number of minutes during the 48-hour measuring period. Of these children, 6 out of 21 did no longer reach these high-normal sensor glucose concentrations after 12 months lifestyle intervention. In addition, a significant decrease in duration of glucose concentrations $\geq 6.7 \mathrm{mmol} / \mathrm{L}(p=0.001)$ was demonstrated after 12 months intervention in the complete group (figure 1 ; table 3 ). At baseline, $36 \%(n=12)$ of the children showed sensor glucose concentrations $\geq 7.8$ $\mathrm{mmol} / \mathrm{L}$ for a number of minutes during the 48-hour measuring period. The duration of exceeding this threshold did not change significantly after 12 months lifestyle intervention $(p=0.408$ ) (figure 1 ; table 3 ). However, in 9 out of 12 of the children that exceeded this threshold at baseline, all sensor glucose concentrations remained below $7.8 \mathrm{mmol} / \mathrm{L}$ after 12 months intervention. In both children exceeding glucose concentrations of 10.0 $\mathrm{mmol} / \mathrm{L}(\mathrm{n}=2)$ at baseline, all sensor glucose concentrations were $<6.7 \mathrm{mmol} / \mathrm{L}$ after 12 months intervention. Furthermore, changes in the opposite direction were also observed, i.e. 8 out of 12 children with glucose concentrations $<6.7 \mathrm{mmol} / \mathrm{L}$ at baseline, exceeded this threshold after 12 months lifestyle intervention. Four of these children did not show a decrease in BMI z-score after the intervention.

Finally, at the low end of the plasma glucose spectrum, 73 percent $(n=24)$ of the children reached sensor glucose concentrations below $3.9 \mathrm{mmol} / \mathrm{L}$ at baseline. The total duration of hypoglycemic sensor glucose concentrations did not change significantly after 12 months intervention ( $p=0.323$ ) (figure 1 ; table 1 ). 


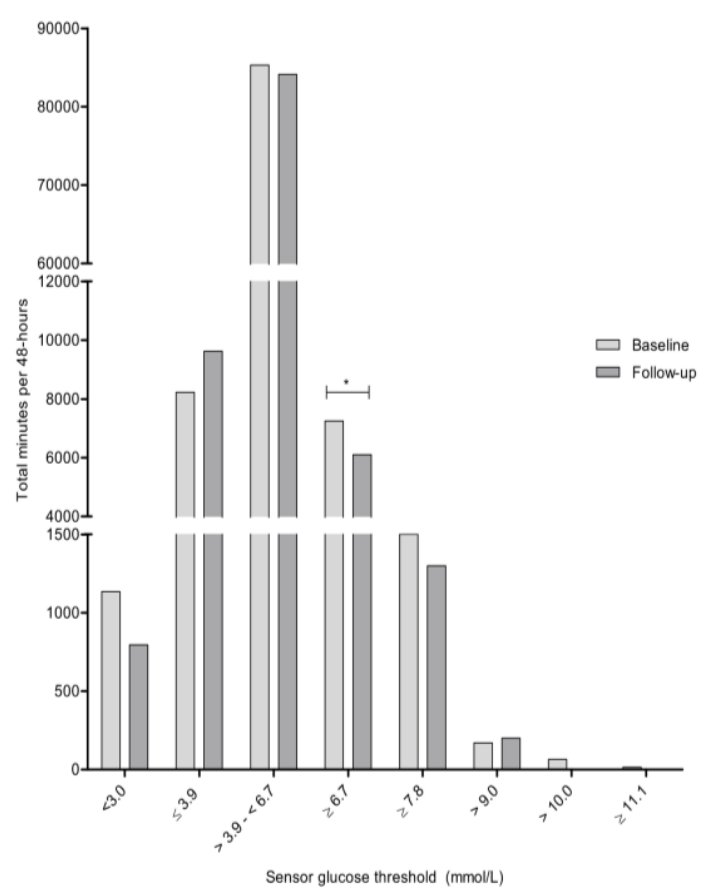

Figure 1. Total minutes per 48 hours that sensor glucose concentrations were within specific glucose thresholds at baseline and after 12 months lifestyle intervention.

Children were divided into two groups, based on whether or not there was a decrease of $\mathrm{BMI} z$-score after one year of lifestyle intervention. In the children who demonstrated a decrease in BMI z-score, the duration of sensor glucose concentrations $\geq 6.7 \mathrm{mmol} / \mathrm{L}$ decreased significantly $(\mathrm{p}=0.009)$, and in the children who demonstrated an increase in $\mathrm{BMI} z$ score there was a trend towards a shorter duration of glucose concentrations $\geq 6.7$ $\mathrm{mmol} / \mathrm{L}(\mathrm{p}=0.060$ ) (table 4). CONGA1 and CONGA2 decreased significantly in children who showed a decrease in BMI $z$-score ( $p=0.021 ; p=0.048$, respectively), whereas there were no significant changes found in children with an increase in BMI z-score $(p=0.552 ; p=0.650$, respectively) (table 4). Sensor glucose measurements stratified for daytime (i.e. 7 AM - 10 PM) and nighttime (i.e. 10 PM - 7 AM) are presented in supplemental table 1 . The median, minimum and maximum sensor glucose concentrations, CV and the duration of glucose concentrations within the different ranges during day- and nighttime, did not differ significantly at baseline compared to after 12 months of lifestyle intervention. The durations of glucose concentrations within the different ranges during day- and nighttime also did not change significantly after lifestyle intervention after stratification for children with and without a decrease in BMI z-score. 

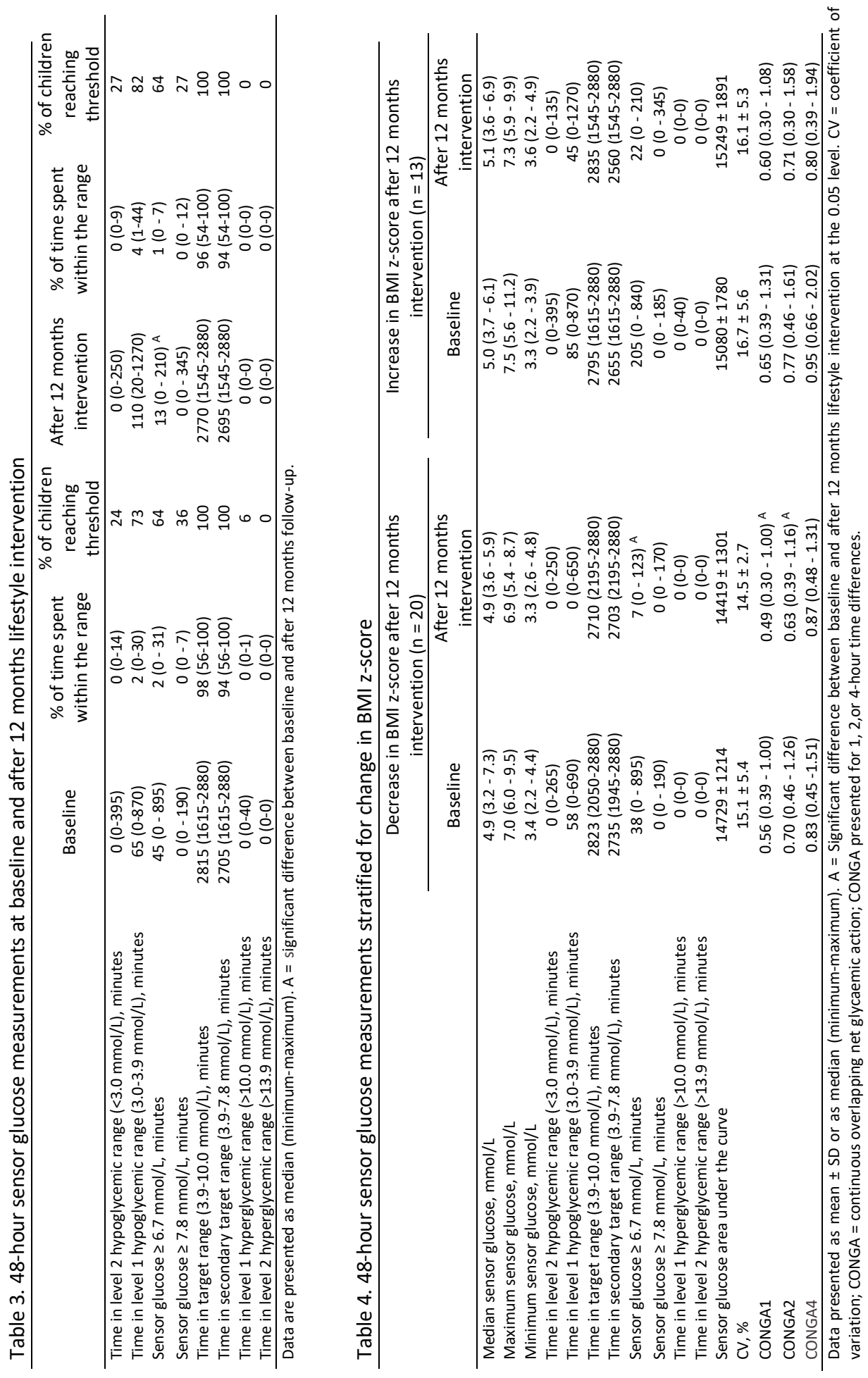


\section{Associations between changes in glucose metabolism and CVD risk after 12 months lifestyle intervention}

After 12 months lifestyle intervention, the delta of the median sensor glucose concentration showed a positive correlation with the delta SBP z-score $(R=0.405$, $p=0.024)$ and delta DBP $z$-score $(R=0.414, p=0.021)$ in the complete group. The delta of the median sensor glucose concentration was not associated with the delta of the anthropometric measurements or the other cardiovascular risk parameters. Delta CONGA1, delta CONGA2, delta CONGA4 and delta CV were not associated with alterations in any of the anthropometric measurements or cardiovascular risk parameters in the complete group. In those children showing a decrease in BMI zscore, there was a positive association between the delta BMI z-score and the delta CONGA1 ( $R=0.601, p=0.005)$, delta CONGA2 ( $R=0.643, p=0.002)$, delta CONGA4 $(R=0.686, p=0.001)$ and delta $C V(R=0.620, p=0.004)$. Moreover, in these children, the delta median sensor glucose concentration was positively associated with the delta LDL$C(R=0.472, p=0.036)$, delta SBP $z$-score $(R=0.598, p=0.005)$, and delta DBP $z$-score $(R=0.605, p=0.005)$. The delta CV was positively associated with delta total cholesterol concentrations $(R=0.453, p=0.045)$ and delta triglyceride concentration $(R=0.457, p=0.043)$. In children with an increase in BMI $z$-score, no associations were found between the delta BMI z-score and alterations in sensor glucose measurements. In these children, the delta maximum sensor glucose concentration showed inverse correlations with delta TC $(R=-0.581, p=0.047)$ and delta LDL-C ( $R=-0.580, p=0.048)$. Correlation coefficients stratified for change in BMI $z$-score are presented in table 5 and table 6 . 
Table 5. Correlation coefficients for changes in patient characteristics and sensor glucose measurements - subgroup analysis for the children with a decrease in BMI z-score

\begin{tabular}{|c|c|c|c|c|c|c|c|c|}
\hline & $\begin{array}{c}\Delta \\
\text { Median } \\
\text { sensor } \\
\text { glucose }\end{array}$ & $\begin{array}{c}\Delta \\
\text { Maximum } \\
\text { sensor } \\
\text { glucose }\end{array}$ & $\begin{array}{c}\Delta \\
\text { Minimum } \\
\text { sensor } \\
\text { glucose }\end{array}$ & $\begin{array}{c}\Delta \\
\text { CONGA1 }\end{array}$ & $\begin{array}{c}\Delta \\
\text { CONGA2 }\end{array}$ & $\begin{array}{c}\Delta \\
\text { CONGA4 }\end{array}$ & $\begin{array}{c}\Delta \\
\mathrm{CV}\end{array}$ & $\begin{array}{c}\Delta \\
\mathrm{AUC}\end{array}$ \\
\hline$\Delta \mathrm{BMI}$ z-score & -0.127 & $0.502^{A}$ & -0.356 & $0.601^{\mathrm{A}}$ & $0.643^{\mathrm{A}}$ & $0.686^{\mathrm{A}}$ & $0.620^{\mathrm{A}}$ & 0.214 \\
\hline$\Delta$ Fasting glucose & -0.067 & -0.241 & -0.342 & -0.095 & -0.076 & 0.03 & 0.350 & -0.416 \\
\hline$\Delta$ Fasting insulin & 0.150 & -0.183 & -0.05 & -0.084 & 0.082 & 0.11 & 0.000 & 0.075 \\
\hline$\triangle$ HOMA-IR & -0.072 & -0.034 & -0.023 & -0.179 & 0.216 & 0.191 & 0.197 & -0.038 \\
\hline$\Delta \mathrm{HbA} 1 \mathrm{c}$ & 0.340 & 0.153 & -0.175 & 0.340 & 0.319 & 0.239 & 0.032 & 0.229 \\
\hline$\Delta$ Glucose 2 -h after glucose load & 0.027 & 0.144 & -0.412 & 0.155 & 0.236 & 0.292 & 0.490 & -0.259 \\
\hline$\Delta$ Total cholesterol & 0.305 & 0.342 & -0.196 & 0.170 & 0.207 & 0.282 & $0.453^{A}$ & 0.162 \\
\hline$\Delta$ LDL-cholesterol & $0.472^{A}$ & 0.150 & 0.029 & 0.166 & 0.045 & 0.021 & 0.111 & 0.214 \\
\hline$\Delta \mathrm{HDL}$-cholesterol & 0.041 & 0.065 & -0.167 & -0.214 & -0.061 & 0.108 & 0.172 & 0.056 \\
\hline$\Delta$ Triglycerides & -0.203 & 0.404 & $-0.445^{A}$ & 0.228 & 0.433 & $0.520^{A}$ & $0.457^{\mathrm{A}}$ & -0.117 \\
\hline$\Delta$ Systolic blood pressure z-score & $0.598^{A}$ & 0.268 & 0.095 & -0.033 & 0.019 & 0.020 & -0.104 & $0.498^{A}$ \\
\hline$\Delta$ Diastolic blood pressure z-score & $0.605^{\mathrm{A}}$ & 0.148 & 0.140 & 0.052 & 0.010 & -0.093 & -0.335 & 0.366 \\
\hline
\end{tabular}

Correlations between variables were determined by Pearson's correlation coefficient or Spearman's correlation analysis, as appropriate. $\mathrm{A}=$ significant correlation. $\Delta=$ delta; HOMA-IR = homeostatic model assessment of insulin resistance; $C V=$ coefficient of variation; CONGA = continuous overlapping net glycaemic action; CONGA presented for 1, 2, or 4-hour time differences; $A U C=$ area under the curve.

Table 6. Correlation coefficients for changes in patient characteristics and sensor glucose measurements - subgroup analysis for the children with an increase in BMI z-score

\begin{tabular}{|c|c|c|c|c|c|c|c|c|}
\hline & $\begin{array}{c}\Delta \\
\text { Median } \\
\text { sensor } \\
\text { glucose }\end{array}$ & $\begin{array}{c}\Delta \\
\text { Maximum } \\
\text { sensor } \\
\text { glucose }\end{array}$ & $\begin{array}{c}\Delta \\
\text { Minimum } \\
\text { sensor } \\
\text { glucose }\end{array}$ & $\begin{array}{c}\Delta \\
\text { CONGA1 }\end{array}$ & $\begin{array}{c}\Delta \\
\text { CONGA2 }\end{array}$ & $\begin{array}{c}\Delta \\
\text { CONGA4 }\end{array}$ & $\begin{array}{c}\Delta \\
\mathrm{CV}\end{array}$ & $\begin{array}{c}\Delta \\
\mathrm{AUC}\end{array}$ \\
\hline$\Delta \mathrm{BMI}$ z-score & -0.302 & 0.195 & 0.045 & -0.172 & -0.050 & -0.044 & 0.003 & -0.177 \\
\hline$\Delta$ Fasting glucose & 0.181 & 0.579 & 0.326 & 0.419 & 0.264 & 0.158 & 0.321 & $0.598^{\mathrm{A}}$ \\
\hline$\Delta$ Fasting insulin & 0.068 & 0.073 & -0.284 & 0.188 & 0.145 & 0.186 & 0.139 & 0.290 \\
\hline$\triangle$ HOMA-IR & 0.025 & 0.182 & 0.036 & 0.441 & 0.357 & 0.324 & 0.343 & 0.388 \\
\hline$\triangle \mathrm{HbA} 1 \mathrm{c}$ & 0.252 & -0.320 & 0.243 & -0.284 & -0.404 & -0.399 & -0.439 & 0.354 \\
\hline$\Delta$ Glucose 2 -h after glucose load & -0.127 & 0.343 & 0.191 & 0.274 & 0.307 & 0.282 & 0.296 & 0.258 \\
\hline$\Delta$ Total cholesterol & -0.330 & $-0.581^{A}$ & -0.278 & -0.506 & -0.379 & -0.250 & -0.473 & -0.568 \\
\hline$\Delta \mathrm{LDL}$-cholesterol & -0.291 & $-0.580^{A}$ & -0.301 & -0.327 & -0.244 & -0.111 & -0.375 & -0.518 \\
\hline$\Delta \mathrm{HDL}$-cholesterol & -0.427 & -0.357 & -0.183 & -0.239 & -0.134 & -0.174 & -0.061 & -0.397 \\
\hline$\Delta$ Triglycerides & 0.195 & 0.273 & 0.116 & -0.213 & -0.163 & -0.171 & -0.101 & 0.183 \\
\hline$\Delta$ Systolic blood pressure z-score & 0.099 & -0.473 & 0.049 & -0.362 & -0.471 & $-0.614^{A}$ & -0.393 & 0.082 \\
\hline$\Delta$ Diastolic blood pressure z-score & 0.052 & $0.789^{A}$ & 0.302 & -0.552 & $-0.671^{A}$ & $-0.728^{A}$ & $-0.664^{A}$ & -0.104 \\
\hline
\end{tabular}

Correlations between variables were determined by Pearson's correlation coefficient or Spearman's correlation analysis, as appropriate. $\mathrm{A}=$ significant correlation. $\Delta=$ delta; HOMA-IR = homeostatic model assessment of insulin resistance; $\mathrm{CV}=$ coefficient of variation; CONGA = continuous overlapping net glycaemic action; CONGA presented for 1, 2, or 4-hour time differences; $A U C=$ area under the curve. 


\section{Discussion}

This is the first study investigating the effect of a long-term lifestyle intervention on glycemic profiles in free-living conditions in children with overweight and (morbid) obesity. Our results demonstrate that the number of minutes that glucose concentrations are high-normal and the glycemic variability calculated as CONGA1 decreased significantly after 12 months intervention. Furthermore, the delta of the median glucose concentrations in free-living conditions was positively associated with the deltas of the SBP and DBP z-score. These associations were only present in children with a decrease in BMI z-score. Our results suggest that an on-going, tailored, outpatient lifestyle intervention can result in improvement of glycemic profiles in free-living conditions and coincides with a decreased CVD risk in children with overweight and (morbid) obesity.

In a previous cross-sectional study in children with overweight and (morbid) obesity, we demonstrated that glycemic profiles in free-living conditions were aberrant (7). This was not just the consequence of excess body weight, since none of the sensor glucose measurements were associated with BMI z-score (7). We now show, not only that a lifestyle intervention improves glycemic profiles, but also that reduction of BMI z-score coincides with improvement of glycemic profiles. The duration of glucose concentrations in the high-normal range and the CONGA1 only improved in children with a decrease in BMI z-score. Notably, associations between the delta of the median sensor glucose concentrations and deltas of the SBP and DBP z-score were only found in the subgroup of children with a decreased BMI Z-score. It is tempting to suggest that a decrease of BMI zscore is the result of lifestyle improvements. Dietary composition and quality as well as physical activity were important aspects of the lifestyle intervention, and are well-known factors interacting with glucose homeostasis (32). As mentioned previously, in adults with T2DM it has been demonstrated that interventions targeting diet or physical activity both resulted in a significant improvement of glycemic variability (15-18). In contrast to these standardized interventions, our intervention at the outpatient clinic was aimed at gradual lifestyle improvements taking into account personal needs and opportunities of each family, resulting in a wide heterogeneity of dietary intake and physical activity. Since these factors were not assessed in detail in this study, we cannot differentiate whether the observed positive effects on glycemic profiles are the result of improvement in weight, improvement of lifestyle, or a combination of both. In future studies it needs to be elucidated which specific modifiable factors contribute to the improvements in glycemic profiles in children with overweight and (morbid) obesity in free-living conditions in order to facilitate a better definition of targets for future intervention strategies. 
Notwithstanding, these results underscore that an on-going, tailored, outpatient lifestyle intervention resulted in beneficial effects on glucose homeostasis and CVD risk in children with a decrease in BMI z-score.

In the current study glycemic variability was assessed using the CONGA and the coefficient of variation. In general, high glucose variability is thought to be harmful for peripheral vascular function (10-13), and brain development in children (14). Currently, reference ranges for glycemic variability as estimated with the CONGA1, 2 and 4 in healthy children with a normal weight are unknown. However, the CV in our study was comparable to the values that were described in a previous study in 57 healthy children (9). Interestingly, the significant improvement of CONGA as shown in our study illustrates that improvement of the glycemic variability is possible in children with overweight and (morbid) obesity via lifestyle adaptations. In addition to CONGA, glycemic control over a longer period of time was evaluated by assessing $\mathrm{HbA} 1 \mathrm{c}$ concentrations. Both, in children demonstrating a decrease and increase in BMI z-score, HbA1c concentrations improved significantly after 12 months lifestyle intervention. Previous studies in adults with T2DM demonstrated that control of postprandial hyperglycemia is a very important contributor to $\mathrm{HbA} 1 \mathrm{c}$ concentrations $(33,34)$. Taking into account the decreased length of time that glucose concentrations were high-normal, the improvement of CONGA1 and 2, and the improvement of $\mathrm{HbA1c}$ concentrations, we hypothesize that this improvement in glucose homeostasis might be due to a reduction in postprandial glucose excursions after 12 months lifestyle intervention.

The exact underlying mechanisms and sequence of events eventually resulting in glucose dysregulation are not fully understood, but there is strong evidence suggesting a link between glucose dysregulation and dyslipidaemia (35). In this study, the delta maximum sensor glucose concentration was inversely associated with delta TC and delta LDL-C in these children. Furthermore, it was shown that the delta of the median glucose concentration was associated with the deltas of the SBP and DBP z-score, only in children with a decrease in BMI z-score. Interestingly, these correlations between the delta of the median glucose concentrations and deltas SBP and DBP z-score were not found for deltas of the CV and CONGA1, CONGA2, or CONGA4. In children with an increase in BMI z-score HbA1c concentrations improved significantly, while cardiovascular risk parameters showed no significant improvements. These results suggest that changes in glucose homeostasis may, but do not necessarily, coincide with changes in cardiovascular risk parameters. 
Due to the long-term follow-up of our intervention we did not include a control group with random assignment of treatment, because it is our sincere opinion that it was ethically not justifiable to keep children in a control program for a prolonged period of time and withhold them from treatment. This can be considered as a limitation to this study. Furthermore, the cohort size of our study might seem small, and therefore affirmation of our findings in larger cohort studies is certainly recommendable. However, considering the current literature and the novelty of investigating sensor glucose measurements in children without having the diagnosis of T2DM, it can also be argued that we have a relevant study population size and results of this study might certainly create awareness that future research is warranted. It would also have been valuable if healthy children with a normal weight were included in this study as a reference population for normality of glycemic profiles, since the current evidence in this population is limited $(9,36)$. Additionally, it would be interesting to investigate which modifiable factors contribute to glycemic profiles in free-living conditions in children with overweight and (morbid) obesity, for example by objectively assessing physical activity using an accelerometer.

\section{Conclusion}

In children with a decrease in BMI z-score, glycemic profiles in free-living conditions improve after 12 months of lifestyle intervention, as demonstrated by a decrease in the duration of time that glucose concentrations are in the high normal range and by the decrease in CONGA1. Changes in median glucose concentrations are associated with changes in SBP and DBP z-score in children with overweight and (morbid) obesity, but only in those who showed a decrease in BMI z-score. These results suggest that a lifestyle intervention can result in improvement of glucose homeostasis and cardiovascular health. Next, long-term follow-up studies are necessary to evaluate whether improvement of glycemic profiles in free-living conditions during childhood results into long-term health benefits. 


\section{References}

1. D'Adamo E, Caprio S. Type 2 diabetes in youth: epidemiology and pathophysiology. Diabetes Care. 2011;34 Suppl 2:S161-5.

2. Freedman DS, Mei Z, Srinivasan SR, Berenson GS, Dietz WH. Cardiovascular risk factors and excess adiposity among overweight children and adolescents: the Bogalusa Heart Study. J Pediatr. 2007;150(1):12-7 e2.

3. Haffner SM, Stern MP, Hazuda HP, Mitchell BD, Patterson JK. Cardiovascular risk factors in confirmed prediabetic individuals. Does the clock for coronary heart disease start ticking before the onset of clinical diabetes? JAMA. 1990;263(21):2893-8.

4. Harris MI, Klein R, Welborn TA, Knuiman MW. Onset of NIDDM occurs at least 4-7 yr before clinical diagnosis. Diabetes Care. 1992;15(7):815-9.

5. Rijks JM, Plat J, Mensink RP, Dorenbos E, Buurman WA, Vreugdenhil A. Children with morbid obesity benefit equally as children with overweight and obesity from an on-going care program. J Clin Endocrinol Metab. 2015:jc20151444.

6. Skinner AC, Perrin EM, Moss LA, Skelton JA. Cardiometabolic Risks and Severity of Obesity in Children and Young Adults. N Engl J Med. 2015;373(14):1307-17.

7. Rijks J, Karnebeek K, van Dijk JW, Dorenbos E, Gerver WJ, Stouthart P, et al. Glycaemic Profiles of Children With Overweight and Obesity in Free-living Conditions in Association With Cardiometabolic Risk. Scientific reports. 2016;6:31892.

8. Chan CL, Pyle L, Newnes L, Nadeau KJ, Zeitler PS, Kelsey MM. Continuous glucose monitoring and its relationship to hemoglobin A1c and oral glucose tolerance testing in obese and prediabetic youth. J Clin Endocrinol Metab. 2015;100(3):902-10.

9. Shah VN, DuBose SN, Li Z, Beck RW, Petesrs AL, Weinstock RS, et al. Continuous Glucose Monitoring Profiles in Healthy Non-Diabetic Participants: A Multicenter Prospective Study. J Clin Endocrinol Metab. 2019.

10. Razavi Nematollahi L, Kitabchi AE, Stentz FB, Wan JY, Larijani BA, Tehrani MM, et al. Proinflammatory cytokines in response to insulin-induced hypoglycemic stress in healthy subjects. Metabolism. 2009;58(4):443-8.

11. Adler GK, Bonyhay I, Failing H, Waring E, Dotson S, Freeman R. Antecedent hypoglycemia impairs autonomic cardiovascular function: implications for rigorous glycemic control. Diabetes. 2009;58(2):360-6.

12. Ceriello A, Esposito K, Piconi L, Ihnat MA, Thorpe JE, Testa R, et al. Oscillating glucose is more deleterious to endothelial function and oxidative stress than mean glucose in normal and type 2 diabetic patients. Diabetes. 2008;57(5):1349-54.

13. Monnier L, Mas E, Ginet C, Michel F, Villon L, Cristol JP, et al. Activation of oxidative stress by acute glucose fluctuations compared with sustained chronic hyperglycemia in patients with type 2 diabetes. Jama. 2006;295(14):1681-7.

14. Mauras N, Mazaika P, Buckingham B, Weinzimer S, White NH, Tsalikian E, et al. Longitudinal assessment of neuroanatomical and cognitive differences in young children with type 1 diabetes: association with hyperglycemia. Diabetes. 2015;64(5):1770-9.

15. Tay J, Luscombe-Marsh ND, Thompson CH, Noakes M, Buckley JD, Wittert GA, et al. A very low-carbohydrate, low-saturated fat diet for type 2 diabetes management: a randomized trial. Diabetes Care. 2014;37(11):2909-18.

16. Mikus CR, Oberlin DJ, Libla J, Boyle LJ, Thyfault JP. Glycaemic control is improved by 7 days of aerobic exercise training in patients with type 2 diabetes. Diabetologia. 2012;55(5):1417-23. 
17. Van Dijk JW, Manders RJ, Canfora EE, Mechelen WV, Hartgens F, Stehouwer CD, et al. Exercise and 24-h glycemic control: equal effects for all type 2 diabetes patients? Med Sci Sports Exerc. 2013;45(4):628-35.

18. Figueira FR, Umpierre D, Casali KR, Tetelbom PS, Henn NT, Ribeiro JP, et al. Aerobic and combined exercise sessions reduce glucose variability in type 2 diabetes: crossover randomized trial. PLoS One. 2013;8(3):e57733.

19. Slover RH, Tryggestad JB, DiMeglio LA, Fox LA, Bode BW, Bailey TS, et al. Accuracy of a Fourth-Generation Continuous Glucose Monitoring System in Children and Adolescents with Type 1 Diabetes. Diabetes Technol Ther. 2018;20(9):576-84.

20. Dovc K, Cargnelutti K, Sturm A, Selb J, Bratina N, Battelino T. Continuous glucose monitoring use and glucose variability in pre-school children with type 1 diabetes. Diabetes Res Clin Pract. 2019;147:76-80.

21. Patton SR, Noser AE, Youngkin EM, Majidi S, Clements MA. Early Initiation of Diabetes Devices Relates to Improved Glycemic Control in Children with Recent-Onset Type 1 Diabetes Mellitus. Diabetes Technol Ther. 2019;21(7):379-84.

22. Schonbeck $Y$, Talma H, van Dommelen P, Bakker B, Buitendijk SE, Hirasing RA, et al. Increase in prevalence of overweight in Dutch children and adolescents: a comparison of nationwide growth studies in 1980, 1997 and 2009. PLoS One. 2011;6(11):e27608.

23. Cole TJ, Lobstein T. Extended international (IOTF) body mass index cut-offs for thinness, overweight and obesity. Pediatr Obes. 2012;7(4):284-94.

24. Fredriks AM, van Buuren S, Fekkes M, Verloove-Vanhorick SP, Wit JM. Are age references for waist circumference, hip circumference and waist-hip ratio in Dutch children useful in clinical practice? Eur J Pediatr. 2005;164(4):216-22.

25. C S. Etniciteit: Definitie en gegevens. In: Volksgezondheid Toekomst Verkenning. Nationaal Kompas Volksgezondheid Bilthoven: RIVM. 2012.

26. American Diabetes A. 2. Classification and Diagnosis of Diabetes. Diabetes Care. 2016;39 Suppl 1:S13-22.

27. Matthews DR, Hosker JP, Rudenski AS, Naylor BA, Treacher DF, Turner RC. Homeostasis model assessment: insulin resistance and beta-cell function from fasting plasma glucose and insulin concentrations in man. Diabetologia. 1985;28(7):412-9.

28. Danne T, Nimri R, Battelino T, Bergenstal RM, Close KL, DeVries JH, et al. International Consensus on Use of Continuous Glucose Monitoring. Diabetes Care. 2017;40(12):1631-40.

29. 2. Classification and Diagnosis of Diabetes: Standards of Medical Care in Diabetes-2018. Diabetes Care. 2018;41(Suppl 1):S13-s27.

30. McDonnell CM, Donath SM, Vidmar SI, Werther GA, Cameron FJ. A novel approach to continuous glucose analysis utilizing glycemic variation. Diabetes Technol Ther. 2005;7(2):253-63.

31. Wuhl E, Witte K, Soergel M, Mehls O, Schaefer F, German Working Group on Pediatric H. Distribution of 24-h ambulatory blood pressure in children: normalized reference values and role of body dimensions. J Hypertens. 2002;20(10):1995-2007.

32. Grimm JJ. Interaction of physical activity and diet: implications for insulin-glucose dynamics. Public Health Nutr. 1999;2(3A):363-8.

33. Monnier L, Lapinski $\mathrm{H}$, Colette $\mathrm{C}$. Contributions of fasting and postprandial plasma glucose increments to the overall diurnal hyperglycemia of type 2 diabetic patients: variations with increasing levels of $\mathrm{HbA}(1 \mathrm{c})$. Diabetes Care. 2003;26(3):881-5.

34. Woerle HJ, Neumann C, Zschau S, Tenner S, Irsigler A, Schirra J, et al. Impact of fasting and postprandial glycemia on overall glycemic control in type 2 diabetes Importance of postprandial glycemia to achieve target $\mathrm{HbA1c}$ levels. Diabetes Res Clin Pract. 2007;77(2):280-5. 
35. Avramoglu RK, Basciano H, Adeli K. Lipid and lipoprotein dysregulation in insulin resistant states. Clin Chim Acta. 2006;368(1-2):1-19.

36. Juvenile Diabetes Research Foundation Continuous Glucose Monitoring Study G, Fox LA, Beck RW, Xing D. Variation of interstitial glucose measurements assessed by continuous glucose monitors in healthy, nondiabetic individuals. Diabetes Care. 2010;33(6):1297-9. 


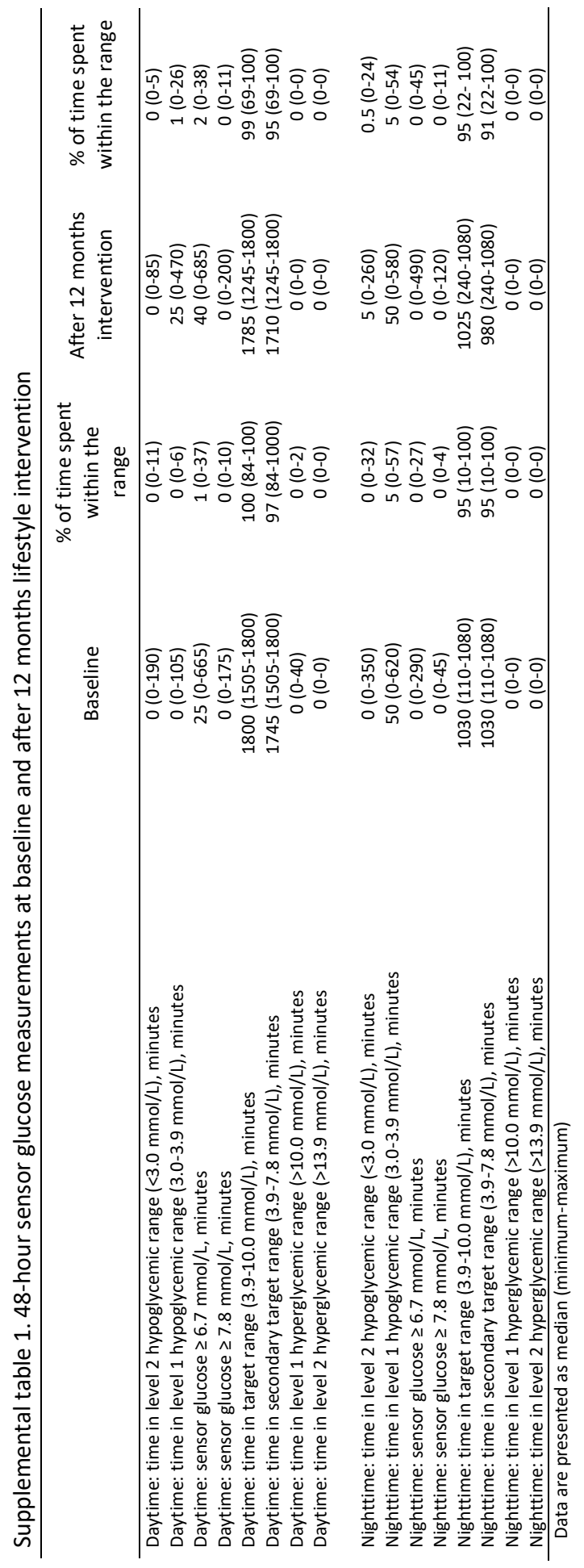




\section{Chapter 4}

\section{Predictors of arterial stiffness in children with overweight, obesity and morbid obesity are sex- specific}

Kylie Karnebeek Jogchum Plat Jesse M. Rijks Ronald P. Mensink Anita C.E. Vreugdenhil 


\section{Abstract}

\section{Background and aim}

Associations between pro-inflammatory markers and vascular cell adhesion molecules with (cardiovascular) comorbidities in children with obesity are unknown. Therefore, to identify predictors of vascular dysfunction in children with overweight, obesity and morbid obesity, we here evaluated relationships between arterial stiffness and cardiovascular risk parameters, including markers for inflammation and endothelial dysfunction.

\section{Methods}

Potential relationships between the augmentation index adjusted for a heart rate of 75 (Alx@75) and blood pressure, lipids, monocyte chemoattractant protein-1, E-selectin, Creactive protein, serum amyloid $A$, soluble intercellular adhesion molecule-1 (sICAM-1), soluble vascular cell adhesion molecule-1, interleukin-6, interleukin-8 and tumor necrosis factor- $\alpha$ (TNF- $\alpha$ ) concentrations were determined using multiple linear regression in 196 children with overweight, obesity and morbid obesity ( $45 \%$ boys).

\section{Results}

The Alx@75 was higher in girls than boys. Height and diastolic blood pressure z-score appeared predictive for Alx@75 in girls, compared to height, sICAM-1 and TNF- $\alpha$ in boys. BMI was not predictive for Alx@75, neither as an absolute value or expressed as deviation from the mean for age and sex (z-score).

\section{Conclusion}

In children with overweight and obesity, no association was found between BMI z-score and Alx@75. Interestingly, the Alx@75 was higher in girls than boys and predictors of Alx@75 were sex-specific. 


\section{Introduction}

The obesity epidemic is associated with a variety of comorbidities such as cardiovascular disease (CVD)(1), which is most likely explained by the presence of obesity-related metabolic disturbances, such as dyslipidaemia, hypertension and glucose metabolism abnormalities, but also by the presence of low-grade inflammation. Adipocyte-derived secretion of pro-inflammatory cytokines, such as tumour necrosis factor (TNF)- $\alpha$ and interleukin (IL)-6, and chemokines like interleukin-8 (IL-8) and monocyte chemoattractant protein (MCP)-1, is higher in individuals with obesity $(2,3)$, just as circulating concentrations of cell adhesion molecules, such as soluble intercellular adhesion molecule 1 (sICAM-1), soluble vascular cell adhesion molecule 1 (sVCAM-1) and E-selectin, which are markers of endothelial dysfunction and predictors of future CVD risk (3). Although cytokines, chemokines and soluble cell adhesion molecules show strong associations with the risk to develop these comorbidities, it is unknown if these parameters contribute to the variation in vascular function in a population of children with overweight, obesity and morbid obesity known to have an elevated CVD risk.

Vascular function can be assessed non-invasively by measuring the augmentation index (Alx), as a measure of arterial stiffness (4). The relationship between arterial stiffness in children and future CVD remains to be determined, but in adults these associations are evident(5). In adult men, but not women, a one SD increase (14\%) in Alx was associated with a more than two-fold increase in cardiovascular mortality after 15 years (6). Currently, it is not yet fully understood which (cardio)metabolic parameters and markers for inflammation and endothelial function contribute to the variation in Alx in children. Previous studies have shown an increased Alx in children with dyslipidaemia (7) and type 2 diabetes (8). However, findings on the association of the Alx with childhood obesity are inconsistent $(7,9,10)$. An elevated Alx has been associated with pro-inflammatory conditions, at least in adults (11). Studies evaluating the Alx in children in relation to inflammation and endothelial dysfunction, have shown an association with hs-CRP (12), but not MCP-1 (13) and were limited to diabetic children. A better understanding of which cardiometabolic and pro-inflammatory parameters contribute to differences in Alx is important, since it can help to identify children with an increased CVD risk and possibly identify targets for intervention. Therefore, we here evaluated the associations between arterial stiffness and markers for inflammation and endothelial dysfunction, in addition to the classical cardiovascular risk parameters in children with overweight, obesity and morbid obesity. 


\section{Materials and methods}

\section{Participants}

This study was conducted within the Centre for Overweight Adolescent and Children's Healthcare (COACH) at the Maastricht University Medical Centre. Children with overweight and obesity are guided in this centre as described previously (14). Before lifestyle intervention, children underwent a comprehensive clinical assessment to diagnose comorbidities. Children starting the intervention program between January 2011 and September 2014, with complete Alx measurement and blood samples for analysis, were included $(n=202)$. Children that reported signs of infection during the clinical assessment were excluded from this study $(n=6)$. The study was conducted in concordance with the Declaration of Helsinki and approved by the medical ethical committee of the Maastricht UMC+. Informed consent was obtained from a parent and/or legal guardian for study participation before the start of the measurements.

\section{Anthropometric measurements}

Weight and height were measured barefoot, in underwear, using a digital scale (Seca) and stadiometer (De Grood Metaaltechniek). Body Mass Index (BMI) z-scores were obtained using a growth analyser (Growth Analyser VE). Children were classified as having overweight, obesity or morbid obesity based on International Obesity Task Force criteria (15). Waist circumference z-scores were determined according to Dutch reference values (16). Tanner puberty stages were determined in 91 children. If possible, pubertal status was predicted based on age according to the results of Susman et al. (17) in the remaining children. In short, cut-off values for pre-pubertal age were set at the lower limit of the 95\% confidence interval for Tanner stage 2 (9.8 years for girls and 10.3 years for boys). Cut-off values for post-pubertal age were set at the upper limit of the $95 \%$ confidence interval for Tanner stage 5 (14.4 years for girls and 15.0 years for boys). With this method, pubertal status was predicted in 53 children. Since age of puberty onset is highly variable, puberty status was considered as missing in children with an age between these cut-off points $(n=52)$.

\section{Cardiovascular risk parameters and glucose metabolism}

Fasting concentrations of serum total cholesterol, HDL-cholesterol, triglycerides, free fatty acids (FFA) and plasma glucose were determined with the Cobas 8000 analyser (Roche) and serum insulin concentrations with the Immulite 1000 (Siemens). LDL cholesterol was calculated using the Friedewald equation (total cholesterol ( $\mathrm{mmol} / \mathrm{L})$ - HDL cholesterol 
$(\mathrm{mmol} / \mathrm{L})$ - triglycerides $(\mathrm{mmol} / \mathrm{L}) / 2)$. The homeostasis model assessment for insulin resistance (HOMA-IR) was calculated (HOMA-IR = fasting glucose $(\mathrm{mmol} / \mathrm{L}) \times$ fasting insulin $(\mu \mathrm{U} / \mathrm{L}) / 22.5)$. Blood pressure was measured approximately 20 times using the Mobil-OGraph NG (I.E.M. GmbH), after which the mean was calculated. Blood pressure z-scores were calculated according reference values for height and sex (18). The Alx was measured after an overnight fast using the Mobil-O-Graph NG. Since the Alx is affected by heart rate, values were standardized to a heart rate of 75 beats per minute (Alx@75).

\section{Markers for inflammation and endothelial dysfunction}

Plasma concentrations of inflammatory markers (II-6, IL-8, MCP-1, and TNF- $\alpha$ ), cellular adhesion molecules (soluble (s) E-selectin, sICAM-1, and SVCAM-1), and the acute phase proteins C-reactive protein (CRP) and serum amyloid $A(S A A)$ were measured with a commercially available Multi Spot ELISA kit (Meso Scale Discovery).

\section{Statistical analysis}

Statistical analyses were performed with IBM SPSS statistics 22.0. Data was tested for normality with the Shapiro-Wilk test and reported as mean \pm standard deviation or as median (interquartile range) depending on the distribution. Differences between sex were analyzed with an independent samples T-test or Mann-Whitney $U$ test, as appropriate. Correlations between variables were determined by Pearson's or Spearman's correlation coefficients, as appropriate. Forward multiple linear regression analysis was performed to evaluate which parameters contributed to the differences in Alx@75. A p-value $<0.05$ was considered statistically significant.

\section{Results}

One hundred and ninety-six children ( 88 boys) were included. Eighteen \% of all children had overweight, $47 \%$ had obesity and $35 \%$ had morbid obesity. Mean age was $12.6 \pm 3.1$ years and was comparable between children with overweight, obesity and morbid obesity. The mean BMI z-score was $3.35 \pm 0.68$. Additional baseline characteristics are shown in tables 1 and 2.

\section{Differences between boys and girls and weight status categories}

BMI z-score was significantly higher in boys $(3.51 \pm 0.67)$ than girls $(3.21 \pm 0.67)$. Despite the higher BMI z-score in boys, the Alx@75 was significantly higher in girls (23.0\% (IQR 
15.0;29.8)) than boys (13.5\% (IQR 5.0;22.0)(p<0.001). Also, CRP and IL-6 concentrations were higher in girls. Compared to the children with overweight, the children with obesity and morbid obesity showed a more disturbed metabolic and inflammatory profile, with lower serum HDL cholesterol concentrations and higher HOMA-IR, CRP, SAA and IL- 6 concentrations. This difference cannot be explained by sex, since all groups contained equal percentages boys and girls.

Table 1. Baseline characteristics of the study participants, stratified for sex

\begin{tabular}{|c|c|c|c|}
\hline & $\begin{array}{c}\text { Total } \\
(n=196)\end{array}$ & $\begin{array}{l}\text { Boys } \\
(n=88)\end{array}$ & $\begin{array}{c}\text { Girls } \\
(n=108)\end{array}$ \\
\hline Age & $12.6 \pm 3.1$ & $12.6 \pm 2.9$ & $12.6 \pm 3.2$ \\
\hline BMI z-score & $3.35 \pm 0.68$ & $3.51 \pm 0.67$ & $3.21 \pm 0.67^{A}$ \\
\hline Waist circumference z-score & $5.81 \pm 2.25$ & $5.83 \pm 2.28$ & $5.80 \pm 2.23$ \\
\hline Height, $\mathrm{cm}$ & $155.6 \pm 15.4$ & $158.0 \pm 16.3$ & $153.6 \pm 14.4$ \\
\hline Prepubertal/pubertal/postpubertal & $48 / 30 / 66$ & $24 / 8 / 21$ & $24 / 22 / 45$ \\
\hline Systolic blood pressure z-score & $0.41 \pm 1.06$ & $0.26 \pm 1.01$ & $0.53 \pm 1.10$ \\
\hline Diastolic blood pressure z-score & $-0.49 \pm 1.14$ & $-0.55 \pm 1.13$ & $-0.43 \pm 1.15$ \\
\hline Total cholesterol, $\mathrm{mmol} / \mathrm{L}$ & $4.49 \pm 0.82$ & $4.41 \pm 0.73$ & $4.56 \pm 0.73$ \\
\hline LDL-cholesterol, $\mathrm{mmol} / \mathrm{L}$ & $2.80(2.20 ; 3.20)$ & $2.60(2.10 ; 3.10)$ & $2.80(2.23 ; 3.20)$ \\
\hline HDL-cholesterol, $\mathrm{mmol} / \mathrm{L}$ & $1.20(1.00 ; 1.400)$ & $1.30(1.00 ; 1.50)$ & $1.20(1.00 ; 1.40)$ \\
\hline Triglycerides, $\mathrm{mmol} / \mathrm{L}$ & $1.06(0.74 ; 1.37)$ & $1.04(0.72 ; 1.34)$ & $1.08(0.78 ; 1.37)$ \\
\hline Free fatty acids, $\mathrm{mmol} / \mathrm{L}$ & $0.65(0.50-0.78)$ & $0.62(0.45 ; 0.80)$ & $0.66(0.52 ; 0.78)$ \\
\hline$H O M A-I R$ & $3.49 \pm 3.24$ & $3.49 \pm 3.70$ & $3.49 \pm 2.97$ \\
\hline Alx@75,\% & $19.5(9.0 ; 27.0)$ & $13.5(5.0 ; 22.0)$ & $23.0(15.0 ; 29.8)^{A}$ \\
\hline$M C P-1, p g / m L$ & $129.1(107.2 ; 163.3)$ & $134.1(109.6 ; 168.1)$ & $125.1(105.3 ; 157.9)$ \\
\hline E-selectin, $n g / m L$ & $14.4(10.0 ; 21.5)$ & $15.5(10.8 ; 22.1)$ & $15.5(10.8 ; 20.6)$ \\
\hline$C R P, \mu g / m L$ & $2.94(1.28 ; 6.54)$ & $2.27(1.01 ; 5.06)$ & $3.34(1.56 ; 8.21)^{A}$ \\
\hline$S A A, \mu g / m L$ & $2.78(1.41 ; 4.68)$ & $2.31(1.40 ; 4.42)$ & $2.93(1.67 ; 4.86)$ \\
\hline$s / C A M-1, n g / m L$ & $474(399 ; 563)$ & $469(396 ; 549)$ & $474(400 ; 593)$ \\
\hline$s V C A M-1, n g / m L$ & $722(612 ; 820)$ & $733(625 ; 826)$ & 705 (601.;819) \\
\hline$I L-6, p g / m L$ & $0.79(0.53 ; 1.17)$ & $0.67(0.49 ; 1.04)$ & $0.95(0.63 ; 1.29)^{A}$ \\
\hline $\mathrm{IL}-8, \mathrm{pg} / \mathrm{mL}$ & $2.83(2.11 ; 4.07)$ & $2.93(2.28 ; 4.19)$ & $2.66(2.02 ; 3.74)$ \\
\hline$T N F-\alpha, p g / m L$ & $2.46(2.14 ; 2.87)$ & $2.48(2.22 ; 2.93)$ & $2.43(2.03 ; 2.84)$ \\
\hline
\end{tabular}

Data presented as mean (SD) or median (Q1-Q3); A = Statistically different between boys and girls, $p<0.05 ; \mathrm{HOMA}-\mathrm{IR}=$ Homeostatic Model Assessment of Insulin Resistance; Alx@75 = Augmentation Index adjusted for a heart rate of 75; MCP-1 = monocyte chemoattractant protein $1 ; \mathrm{CRP}=\mathrm{C}$-reactive protein; SAA = serum amyloid A protein; sICAM-1 = soluble intracellular adhesion molecule-1, sVCAM-1 = soluble vascular cell adhesion molecule-1; IL-6 = interleukin 6; IL-8 = interleukin 8; TNF- $\alpha=$ tumor necrosis factor $\alpha$. 
Table 2. Characteristics of the study participants, stratified for weight status categories

\begin{tabular}{|c|c|c|c|}
\hline & $\begin{array}{l}\text { Overweight } \\
\qquad(n=35)\end{array}$ & $\begin{array}{l}\text { Obese } \\
(n=93)\end{array}$ & $\begin{array}{l}\text { Morbidly obese } \\
\qquad(n=68)\end{array}$ \\
\hline Age & $12.2 \pm 2.9$ & $12.3 \pm 2.8$ & $13.3 \pm 3.5$ \\
\hline Male/Female, $\%$ & $47 / 53 \%$ & $47 / 53 \%$ & $47 / 53 \%$ \\
\hline BMI z-score & $2.36 \pm 0.31$ & $3.21 \pm 0.27$ & $4.04 \pm 0.44^{A, B, C}$ \\
\hline Waist circumference z-score & $3.61 \pm 1.19$ & $5.40 \pm 1.68$ & $7.57 \pm 2.03^{A, B, C}$ \\
\hline Prepubertal/pubertal/postpubertal & $9 / 10 / 8$ & $25 / 14 / 25$ & $14 / 6 / 33$ \\
\hline Systolic blood pressure z-score & $0.01 \pm 0.93$ & $0.44 \pm 1.03$ & $0.56 \pm 1.4$ \\
\hline Diastolic blood pressure z-score & $-0.93 \pm 1.04$ & $-0.43 \pm 1.02$ & $-0.34 \pm 1.28$ \\
\hline Total cholesterol, $\mathrm{mmol} / \mathrm{L}$ & $4.38 \pm 0.88$ & $4.43 \pm 0.77$ & $4.64 \pm 0.83$ \\
\hline LDL-cholesterol, $\mathrm{mmol} / \mathrm{L}$ & $2.60(2.10 ; 3.00)$ & $2.60(2.10 ; 3.10)$ & $2.90(2.40 ; 3.38)$ \\
\hline HDL-cholesterol, $\mathrm{mmol} / \mathrm{L}$ & $1.40(1.20 ; 1.50)$ & $1.25(1.00 ; 1.40)$ & $1.10(1.00 ; 1.30)^{\mathrm{B}}$ \\
\hline Triglycerides, $\mathrm{mmol} / \mathrm{L}$ & $1.00(0.72 ; 1.22)$ & $1.04(0.73 ; 1.37)$ & $1.14(0.82 ; 1.51)$ \\
\hline Free fatty acids, $\mathrm{mmol} / \mathrm{L}$ & $0.71(0.54 ; 0.81)$ & $0.66(0.51 ; 0.81)$ & $0.61(0.46 ; 0.72)$ \\
\hline$H O M A-I R$ & $2.14 \pm 1.16$ & $3.14 \pm 2.58$ & $4.66 \pm 4.24^{\mathrm{B}, \mathrm{C}}$ \\
\hline Alx@75,\% & $21.0(5.0 ; 31.0)$ & $18.0(10.0 ; 27.0)$ & $19.5(8.5 ; 26.0)$ \\
\hline$M C P-1, p g / m L$ & $120.3(100.2 ; 155.6)$ & $130.7(107.1 ; 167.0)$ & $132.9(109.3 ; 166.9)$ \\
\hline E-selectin, $n g / m L$ & $14.0(9.7 ; 21.2)$ & $15.1(9.4 ; 20.6)$ & $14.4(10.3 ; 23.3)$ \\
\hline$C R P, \mu g / m L$ & $1.82(0.60 ; 4.52)$ & $2.24(1.03 ; 5.50)$ & $4.57(2.36 ; 9.82)^{B, C}$ \\
\hline$S A A, \mu g / m L$ & $2.00(1.24 ; 3.53)$ & $2.19(1.33 ; 4.58)$ & $3.71(2.03 ; 5.54)^{B, C}$ \\
\hline$s / C A M-1, \mu g / m L$ & $475(383 ; 587)$ & $488(427 ; 584)$ & $455(393 ; 544)$ \\
\hline$s V C A M-1, \mu g / m L$ & $698(627 ; 826)$ & $760(612 ; 831)$ & $677(597 ; 804)$ \\
\hline$I L-6, p g / m L$ & $0.72(0.46 ; 0.98)$ & $0.72(0.49 ; 1.14)$ & $0.98(0.63 ; 1.39)^{B, C}$ \\
\hline$I L-8, p g / m L$ & $3.06(2.36 ; 4.56)$ & $2.85(2.21 ; 4.28)$ & $2.49(1.97 ; 3.54)$ \\
\hline$T N F-\alpha, p g / m L$ & $2.42(2.24 ; 2.91)$ & $2.46(2.12 ; 2.88)$ & $2.53(2.14 ; 2.80)$ \\
\hline
\end{tabular}

Data presented as mean (SD) or median (Q1-Q3); A=Statistically different between overweight and obese, $p<0.0167 ; \mathrm{B}=$ Statistically different between overweight and morbidly obese, $p<0.0167 ; \mathrm{C}=$ Statistically different between obese and morbidly obese, $p<0.0167$; HOMA-IR = Homeostatic Model Assessment of Insulin Resistance; AIx@75 = Augmentation Index adjusted for a heart rate of 75; MCP-1 = monocyte chemoattractant protein 1; CRP = C-reactive protein; SAA = serum amyloid A protein; sICAM-1 = soluble intracellular adhesion molecule-1, sVCAM-1 = soluble vascular cell adhesion molecule-1; IL-6 = interleukin 6; IL-8 = interleukin 8; TNF- $\alpha$ $=$ tumor necrosis factor $\alpha$.

\section{Associations between arterial stiffness and cardiometabolic parameters, sex, puberty and inflammatory markers}

To identify parameters contributing to the differences in the Alx@75, forward multiple regression analyses were performed. An overview of the different regression models and the included parameters is shown in supplementary table 1. First, we evaluated which of the classical risk parameters (lipids and lipoproteins, blood pressure, HOMA-IR, BMI and waist circumference) contributed to variation in Alx@75. This first regression model identified height $(\mathrm{cm} ; \beta=-0.363 ; p<0.001)$, sex (male; $\beta=-6.047 ; p<0.001$ ) and diastolic blood pressure $(\beta=0.276 ; p=0.015)$ as significantpredictors (table 3 ) and explained $34 \%$ of the variance in Alx@75. 
Since normal values for BMI, waist-circumference and blood pressure in children are sex and age dependent, the same regression modeling procedure was performed with zscores for these parameters to assess their contribution expressed as deviation from the healthy mean. This second model identified height $(\mathrm{cm} ; \beta=-0.347 ; p<0.001)$, sex (male; $\beta=-7.196 ; p<0.001)$ and diastolic blood pressure $z$-score $(\beta=1.612 ; p=0.013)$ as significant predictors (table 4), explaining 37\% of the variance in Alx@75, and was highly comparable to model 1 . Further stratification for gender in this second model identified height $(\mathrm{cm} ; \beta=-0.313 ; p<0.001)$ and diastolic blood pressure $z$-score $(\beta=1.795 ; p=0.034)$ as significant predictors in girls, explaining $22 \%$ of the variance. In boys, height was the only significant predictor in this model $(\mathrm{cm} ; \beta=0.336 ; p<0.001)$, explaining $27 \%$ of the variance in the Alx@75.

Since it is possible that the differences in the Alx@75 between boys and girls are related to puberty stage, this was also examined. However, stratification for puberty status showed that the difference in Alx@75 between boys and girls was already present in prepubertal children (supplementary table 2). Moreover, the sex difference increased during and after puberty, i.e. the difference between boys and girls in the Alx@75 was 6.5\% in pre-pubertal children, $7.5 \%$ in pubertal and $18.0 \%$ for post-pubertal children, so we cannot fully exclude the role of puberty. However, a similar pattern was also seen for the sex difference in height, but this difference only reached significance in post-pubertal children. We further evaluated to what extent these differences in height could explain the difference in the Alx@75 between boys and girls. Regression analysis showed that the Alx@75 decreases with 0.35\% for each centimeter increase in height, regardless of sex (table 4). Since boys were taller than girls, this could partially explain the observed sex differences. Further elaboration showed that the height difference explained $1.9 \%(5.4 \mathrm{~cm}$ $x$ 0.35\%) of the 6.5\% difference in the Alx@75 between pre-pubertal boys and girls, 2.3\% $(6.6 \mathrm{~cm} \times 0.35 \%)$ of the $7.5 \%$ difference in pubertal children and $4.4 \%$ (12.8 $\mathrm{cm} \times 0.35 \%)$ of the $18.0 \%$ difference for post-pubertal children (supplementary table 2 ).

Next, we examined the influence of inflammatory status on variation in Alx@75. Since plasma CRP and IL- 6 concentrations were significantly higher in girls, we first compared the Alx@75 values between CRP and IL-6 tertiles. However, differences in the Alx@75 between these tertiles did not reach statistical significance for girls or boys (supplementary table 3). Next, correlations between the Alx@75 and all the markers for inflammation and endothelial function were analyzed. Overall, the Alx@75 correlated significantly with E-selectin, sICAM-1 and IL-6 (table 5). Therefore, all markers were also included in the regression analyses. In model 3 , height $(\mathrm{cm} ; \beta=-0.292 ; p<0.001)$, sex (male; $\beta=-6.645 ; p<0.001)$, diastolic blood pressure $(\beta=0.303 ; p=0.006)$, sICAM-1 
( $\mathrm{ng} / \mathrm{mL} ; \beta=0.020 ; p=0.009$ ) and $\mathrm{HDL}$ cholesterol ( $\mathrm{mmol} / \mathrm{L} ; \beta=5.736 ; p=0.031$ ) concentrations were identified as significant contributors, explaining 39\% of the variance in Alx@75 (table 3).

The same modeling procedure was performed using z-scores for BMI, waist-circumference and blood pressure. In this final model 4 (table 4$)$, height $(\mathrm{cm} ; \beta=-0.349 ; p<0.001)$, sex (male; $\beta=-6.109 ; p<0.001)$, diastolic blood pressure $z$-score $(\beta=1.645 ; p=0.008)$, sICAM-1 (ng/ $\mathrm{mL} ; \beta=0.025 ; \mathrm{p}=0.001)$, IL-6 (pg/mL; $\beta=3.12 ; \mathrm{p}=0.013)$ and TNF- $\alpha(\mathrm{pg} / \mathrm{mL} ; \beta=-3.884 ; p=0.003)$ contributed significantly to the Alx@75, together explaining 44\% of the variation in Alx@75. After stratification for sex, the contribution of inflammation and endothelial dysfunction was only significant in boys, identifying height $(\mathrm{cm} ; \beta=-0.294 ; p<0.001)$, sICAM-1 ( $\mathrm{ng} / \mathrm{mL} ; \beta=0.039 ; \mathrm{p}=0.001)$ and TNF- $\alpha(\mathrm{pg} / \mathrm{mL} ; \beta=-3.979 ; \mathrm{p}=0.049)$ as significant predictors. In girls, addition of the markers for inflammation and endothelial dysfunction to the regression model did not identify additional significant predictors for the Alx@75, besides the diastolic blood pressure $z$-score $(\beta=1.795 ; p=0.034)$ and height $(\mathrm{cm}$; $\beta=-0.313 ; p<0.001)$.

Table 3. Forward regression models for variables predicting Alx@75

\begin{tabular}{lcclcc}
\multicolumn{1}{c}{ Model 1 } & $\beta$ & $\begin{array}{c}p \text { - } \\
\text { value }\end{array}$ & & Model 3 & $p$-value \\
\hline $\mathrm{R}^{2}=0.337$ & & & $\mathrm{R}^{2}=0.385$ & \\
& & & & & \\
Height, $\mathrm{cm}$ & -0.363 & $<0.001$ & Height, $\mathrm{cm}$ & -0.292 & $<0.001$ \\
Sex, male & -6.047 & $<0.001$ & Sex, male & -6.645 & $<0.001$ \\
Diastolic blood pressure & 0.276 & 0.015 & Diastolic blood pressure & 0.303 & 0.006 \\
& & & sICAM-1, $\mathrm{ng} / \mathrm{mL}$ & 0.020 & 0.009 \\
& & & HDL cholesterol, mmol/L & 5.736 & 0.031 \\
\hline
\end{tabular}

Model 1 (only significant predictors are presented in table): BMI, height, sex, waist circumference, systolic blood pressure, diastolic blood pressure, total cholesterol, HDL cholesterol, LDL cholesterol, triglycerides, FFA, HOMAIR. Model 3 is model 1 + MCP-1, E-selectin, CRP, SAA, sICAM-1, sVCAM-1, IL-6, IL-8 TNF- $\alpha$. $\beta$ 's shown are unstandardised. 
Table 4. Forward regression models for variables predicting Alx@75

\begin{tabular}{|c|c|c|c|c|c|}
\hline Model 2 & $\beta$ & $\begin{array}{c}p- \\
\text { value }\end{array}$ & Model 4 & $\beta$ & $p$-value \\
\hline$R^{2}=0.367$ & & & $R^{2}=0.441$ & & \\
\hline Height, $\mathrm{cm}$ & -0.347 & $<0.001$ & Height, $\mathrm{cm}$ & -0.349 & $<0.001$ \\
\hline Sex, male & -7.196 & $<0.001$ & Sex, male & -6.109 & $<0.001$ \\
\hline \multirow[t]{4}{*}{ Diastolic blood pressure z-score } & 1.612 & 0.013 & Diastolic blood pressure z-score & 1.645 & 0.008 \\
\hline & & & sICAM-1, ng/mL & 0.025 & 0.001 \\
\hline & & & $I L-6, p g / m L$ & 3.120 & 0.013 \\
\hline & & & $T N F-\alpha, p g / m L$ & -3.884 & 0.003 \\
\hline
\end{tabular}

Model 2 (only significant predictors are presented in table): BMI z-score, height, sex, waist circumference zscore, systolic blood pressure z-score, diastolic blood pressure z-score, total cholesterol, HDL cholesterol, LDL cholesterol, triglycerides, FFA, HOMA-IR. Model 4 is model 2 + MCP-1, E-selectin, CRP, SAA, sICAM-1, sVCAM-1, IL-6, IL-8 TNF- $\alpha$. $\beta$ 's shown are unstandardised.

\section{Discussion}

It is generally accepted that a higher Alx@75 predicts future CVD risk. In this study, we showed that the Alx@75 is higher in girls with overweight and obesity as compared to boys. Moreover, the Alx@75 appeared comparable between children with overweight, obesity and morbid obesity. To the best of our knowledge, this is the first study to evaluate the additional value of including a wide panel of markers reflecting inflammation and vascular dysfunction to classical cardiovascular risk parameters to explain differences in arterial stiffness in boys and girls with overweight, obesity and morbid obesity. We here show that these markers as well as height were significantly related to the variation in Alx@75 in boys. In girls, only height and diastolic blood pressure z-score, but not the markers reflecting inflammation or vascular dysfunction, contributed significantly to the variation in Alx@75.

In our study, the markers for inflammation and endothelial function, as well as height, were significantly related to the variation in Alx@75 in boys. In girls, only height and diastolic blood pressure z-score contributed significantly to the variation in Alx@75. Since normal values for BMI, waist-circumference and blood pressure in children are dependent on sex and age, regression models to evaluate the predictors of variation in Alx@75 were performed with both the absolute values and values expressed as deviation from the normal mean for age and sex (e.g. z-scores). Our results show that BMI, waist circumference and systolic blood pressure were not identified as significant predictors for AIX@75, regardless of using the absolute values or z-scores. Diastolic blood pressure was identified as a significant predictor for the Alx@75, both as an absolute value and as zscore. Altogether, our results lead to the conclusion that BMI, waist circumference and 
systolic blood pressure are not important contributors to Alx@75 in children with obesity, both absolutely and as z-scores. Moreover, it shows that height matters in both boys and girls, whereas additional predictors of Alx@75 were sex-specific. A schematic representation of the different factors contributing to the Alx@75 is summarized in Figure 1.

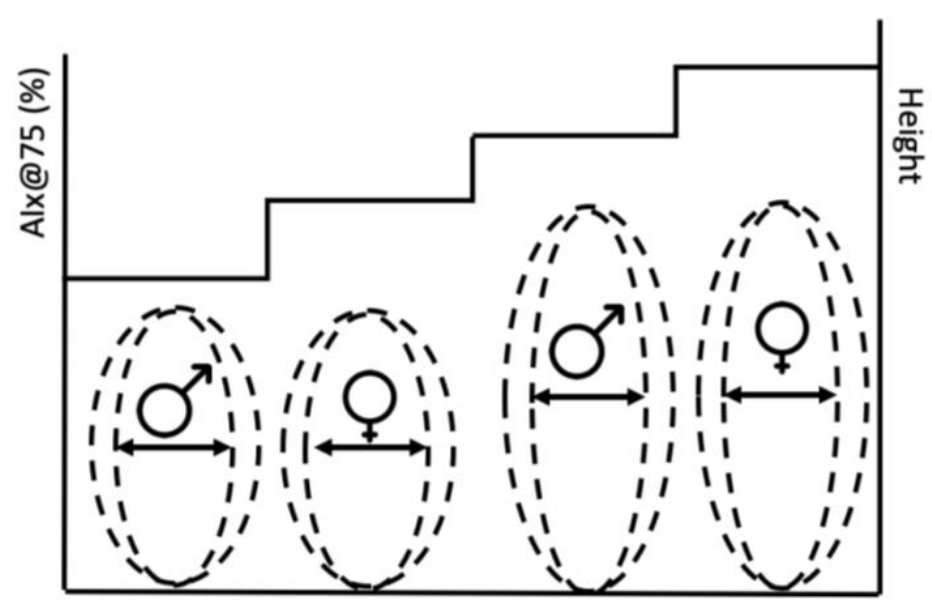

Figure 1. A schematic representation of the effect of BMI and height on the Alx@75, depending on sex. The Alx@75 is higher in taller individuals and in females, regardless of BMI (z-score), and is further influenced by (sexspecific) factors.

In our study, the Alx@75 did not differ between children with overweight, obesity or morbid obesity. A post-hoc power analysis identified that, with our sample size, a power of 0.90 , an SD of 11.7 and $\alpha$ of 0.01, we could have detected a difference in Alx@75 of at least 4.1 between children with overweight, obesity and morbid obesity. Previous studies have shown Alx@75 difference of 8.4 and 5.3 between healthy children and children with respectively diabetes type 1 or hypertension $(19,20)$. These studies suggest that clinically relevant differences in Alx@75 in children are quite large and that, if there was a true group difference to be found in our study, there was enough power to identify such a difference. A previous study by Hudson et al. also found no difference in the Alx between lean children and children with overweight or obesity (10). 
It is interesting to consider the reason why children with obesity do not show an increased Alx, especially since it is not questioned that these children have an elevated CVD risk profile (21). At least in adults, an increased Alx is predictive for (future) CVD (22). Devallance et al. (23) have postulated that it could be the result of dilatation of the arteries in people with obesity. This should not be interpreted as if measuring Alx in individuals with obesity has no value, as we and others clearly showed correlations between Alx and other CVD risk parameters like blood pressure and cholesterol levels (24).

Our study showed a pronounced difference in Alx@75 between boys and girls. A similar sex difference has previously been described in several studies, in both children and adults. This finding was attributed, at least partly, to height differences between men and women. In taller individuals it takes the reflected waves longer to return and therefore the Alx is lower. Height differences also contributed to variation in the Alx@75 in our population, but the sex difference in Alx@75 remained even after correcting for height. Previously, Ayer et al. (25) showed a significantly higher Alx@75 in 8-year old girls compared with boys with a similar height and age, as a result of earlier wave reflection. This excludes the possible explanation that differences might be caused by hormonal differences, since these are not yet evident in 8-year-olds. Moreover, we also found a difference in the Alx@75 between pre-pubertal boys and girls. However, Hayward and Kelly (26) showed a comparable Alx for children younger than ten years, with an increasing sex difference in the Alx with older age, becoming significant from 30 years onwards. The authors postulated that the increased Alx in women might contribute to the sex difference in the prevalence of hypertrophic heart failure. Torjesen et al. (27) described an important role for wave morphology to explain the sex difference in the Alx, where adjustment for wave form characteristics halved the effect of sex on the Alx. Gatzka et al. (28) suggested that smaller and stiffer vessels in women, indicated by an increased pulse pressure, explained the difference in the Alx. Kim et al. (29) and Shiva Kumar et al. (30) showed different contributing effects of several metabolic and inflammatory parameters on the Alx in men and women. Altogether these results suggest several possible co-existing mechanisms to explain the difference in the Alx between boys and girls beside the contribution of height, such as sex-specific characteristics of the cardiovascular system, further influenced by metabolic parameters.

We evaluated for the first time, the contribution of a wide panel of markers for inflammation and endothelial function to differences in the Alx@75 in children with overweight, obesity and morbid obesity. sICAM-1 and TNF- $\alpha$ contributed significantly to differences in the Alx@75 and explained an additional 7\% of the variance, but only in 
boys. Although an association between (hs-)CRP and the Alx has been reported $(11,12)$, studies on the association of such a wide panel of markers with the Alx are scarce and mainly focused on adults. A review by Dulai et al. reported conflicting results on the effect of treatment with TNF- $\alpha$ antagonists on arterial stiffness in arthritis patients, but overall the results suggested that treatment might have a beneficial effect (31). Additional studies are needed to replicate our findings and further explore explanations for an inverse association between the Alx@75 and TNF- $\alpha$.

Despite having higher CRP and IL-6 concentrations, these and other markers for inflammation and endothelial dysfunction did not contribute significantly to the Alx@75 in our population of girls. In addition to height, only diastolic blood pressure z-score contributed significantly to the Alx@75 in girls. On average, blood pressure was within the normotensive range for both sexes. These results show that the parameters contributing to variations in the Alx@75 differ between boys and girls. In girls, additional parameters such as (structural) characteristics of the cardiovascular system, may play a more important role in explaining differences in the Alx@75 than inflammation and endothelial dysfunction.

Additional studies are needed to explore the difference in the Alx@75 between boys and girls and the contribution of cardiometabolic parameters, inflammation and endothelial dysfunction as well as other sex-specific differences. Reference values from healthy lean children based on sex and height are necessary to determine to what extent the values from at-risk populations like the children in our $\mathrm{COACH}$ program are abnormal. Since factors related to the Alx@75 may differ in boys and girls, this might ask for sex-specific tailored intervention strategies. This is in line with the increasing awareness in adult populations that targets for interventions aiming to lower CVD risk are different in men and women (32).

\section{Conclusion}

In children with overweight and obesity, the Alx@75 was significantly higher in girls. BMI was not a predictor for Alx@75, neither as an absolute value or expressed as deviation from the normal mean for age and sex (z-score). Height and sex were clear predictors for the Alx@75, whereas additional predictors of the Alx@75 were sex-specific. 


\section{References}

1. Van Gaal LF, Mertens IL, De Block CE. Mechanisms linking obesity with cardiovascular disease. Nature. 2006;444(7121):875-80.

2. Makki K, Froguel P, Wolowczuk I. Adipose tissue in obesity-related inflammation and insulin resistance: cells, cytokines, and chemokines. ISRN Inflamm. 2013;2013:139239.

3. Adrielle Lima Vieira R, Nascimento de Freitas R, Volp AC. Adhesion molecules and chemokines; relation to anthropometric, body composition, biochemical and dietary variables. Nutr Hosp. 2014;30(2):223-36.

4. Jain S, Khera R, Corrales-Medina VF, Townsend RR, Chirinos JA. "Inflammation and arterial stiffness in humans". Atherosclerosis. 2014;237(2):381-90.

5. Vlachopoulos C, Aznaouridis K, O'Rourke MF, Safar ME, Baou K, Stefanadis C. Prediction of cardiovascular events and all-cause mortality with central haemodynamics: a systematic review and meta-analysis. European heart journal. 2010;31(15):1865-71.

6. Wang KL, Cheng HM, Sung SH, Chuang SY, Li CH, Spurgeon HA, et al. Wave reflection and arterial stiffness in the prediction of 15-year all-cause and cardiovascular mortalities: a community-based study. Hypertension. 2010;55(3):799-805.

7. Nunez F, Martinez-Costa C, Sanchez-Zahonero J, Morata J, Chorro FJ, Brines J. Carotid artery stiffness as an early marker of vascular lesions in children and adolescents with cardiovascular risk factors. Rev Esp Cardiol. 2010;63(11):1253-60.

8. Urbina EM, Kimball TR, Khoury PR, Daniels SR, Dolan LM. Increased arterial stiffness is found in adolescents with obesity or obesity-related type 2 diabetes mellitus. J Hypertens. 2010;28(8):1692-8.

9. Lurbe E, Torro I, Garcia-Vicent C, Alvarez J, Fernandez-Fornoso JA, Redon J. Blood pressure and obesity exert independent influences on pulse wave velocity in youth. Hypertension. 2012;60(2):550-5.

10. Hudson LD, Rapala A, Khan T, Williams B, Viner RM. Evidence for contemporary arterial stiffening in obese children and adolescents using pulse wave velocity: A systematic review and meta-analysis. Atherosclerosis. 2015;241(2):376-86.

11. Kampus P, Kals J, Ristimae T, Fischer K, Zilmer M, Teesalu R. High-sensitivity C-reactive protein affects central haemodynamics and augmentation index in apparently healthy persons. Journal of hypertension. 2004;22(6):1133-9.

12. Li X, Deng YP, Yang M, Wu YW, Sun SX, Sun JZ. Low-Grade Inflammation and Increased Arterial Stiffness in Chinese Youth and Adolescents with Newly-Diagnosed Type 2 Diabetes Mellitus. J Clin Res Pediatr Endocrinol. 2015;7(4):268-73.

13. Zineh I, Beitelshees AL, Silverstein JH, Haller MJ. Serum monocyte chemoattractant protein-1 concentrations associate with diabetes status but not arterial stiffness in children with type 1 diabetes. Diabetes care. 2009;32(3):465-7.

14. Rijks JM, Plat J, Mensink RP, Dorenbos E, Buurman WA, Vreugdenhil AC. Children With Morbid Obesity Benefit Equally as Children With Overweight and Obesity From an Ongoing Care Program. The Journal of clinical endocrinology and metabolism. 2015;100(9):3572-80.

15. Cole TJ, Lobstein T. Extended international (IOTF) body mass index cut-offs for thinness, overweight and obesity. Pediatr Obes. 2012;7(4):284-94.

16. Fredriks AM, van Buuren S, Fekkes M, Verloove-Vanhorick SP, Wit JM. Are age references for waist circumference, hip circumference and waist-hip ratio in Dutch children useful in clinical practice? Eur J Pediatr. 2005;164(4):216-22.

17. Susman EJ, Houts RM, Steinberg L, Belsky J, Cauffman E, Dehart G, et al. Longitudinal development of secondary sexual characteristics in girls and boys between ages $91 / 2$ and 151/2 years. Arch Pediatr Adolesc Med. 2010;164(2):166-73. 
18. Wuhl E, Witte K, Soergel M, Mehls O, Schaefer F. Distribution of 24-h ambulatory blood pressure in children: normalized reference values and role of body dimensions. Journal of hypertension. 2002;20(10):1995-2007.

19. Duarte SV, de Souza Rajao J, Pinho JF, Dos Santos LM, Alves-Neves CM, Magalhaes GS, et al. Changes in aortic pulse wave components, pulse pressure amplification, and hemodynamic parameters of children and adolescents with type 1 diabetes. Pediatric diabetes. 2019;20(2):202-9.

20. Tokgoz ST, Yilmaz D, Tokgoz Y, Celik B, Bulut Y. The evaluation of arterial stiffness of essential hypertension and white coat hypertension in children: a case-control study. Cardiology in the young. 2018;28(3):403-8.

21. Skinner AC, Perrin EM, Moss LA, Skelton JA. Cardiometabolic Risks and Severity of Obesity in Children and Young Adults. The New England journal of medicine. 2015;373(14):130717.

22. Hayashi T, Nakayama Y, Tsumura K, Yoshimaru K, Ueda H. Reflection in the arterial system and the risk of coronary heart disease. Am J Hypertens. 2002;15(5):405-9.

23. DeVallance E, Fournier SB, Donley DA, Bonner DE, Lee K, Frisbee JC, et al. Is obesity predictive of cardiovascular dysfunction independent of cardiovascular risk factors? Int J Obes (Lond). 2015;39(2):244-53.

24. Wilkinson IB, Prasad K, Hall IR, Thomas A, MacCallum H, Webb DJ, et al. Increased central pulse pressure and augmentation index in subjects with hypercholesterolemia. Journal of the American College of Cardiology. 2002;39(6):1005-11.

25. Ayer JG, Harmer JA, Marks GB, Avolio A, Celermajer DS. Central arterial pulse wave augmentation is greater in girls than boys, independent of height. Journal of hypertension. 2010;28(2):306-13.

26. Hayward CS, Kelly RP. Gender-related differences in the central arterial pressure waveform. Journal of the American College of Cardiology. 1997;30(7):1863-71.

27. Torjesen AA, Wang N, Larson MG, Hamburg NM, Vita JA, Levy D, et al. Forward and backward wave morphology and central pressure augmentation in men and women in the Framingham Heart Study. Hypertension (Dallas, Tex : 1979). 2014;64(2):259-65.

28. Gatzka CD, Kingwell BA, Cameron JD, Berry KL, Liang YL, Dewar EM, et al. Gender differences in the timing of arterial wave reflection beyond differences in body height. J Hypertens. 2001;19(12):2197-203.

29. Kim JY, Park JB, Kim DS, Kim KS, Jeong JW, Park JC, et al. Gender Difference in Arterial Stiffness in a Multicenter Cross-Sectional Study: The Korean Arterial Aging Study (KAAS). Pulse (Basel, Switzerland). 2014;2(1-4):11-7.

30. Shiva Kumar P, Medina-Lezama J, Morey-Vargas O, Zamani P, Bolanos-Salazar JF, Chirinos DA, et al. Prospective risk factors for increased central augmentation index in men and women. American journal of hypertension. 2015;28(1):121-6.

31. Dulai R, Perry M, Twycross-Lewis R, Morrissey D, Atzeni F, Greenwald S. The effect of tumor necrosis factor-alpha antagonists on arterial stiffness in rheumatoid arthritis: a literature review. Semin Arthritis Rheum. 2012;42(1):1-8.

32. Appelman $Y$, van Rijn BB, Ten Haaf ME, Boersma E, Peters SA. Sex differences in cardiovascular risk factors and disease prevention. Atherosclerosis. 2015;241(1):211-8. 


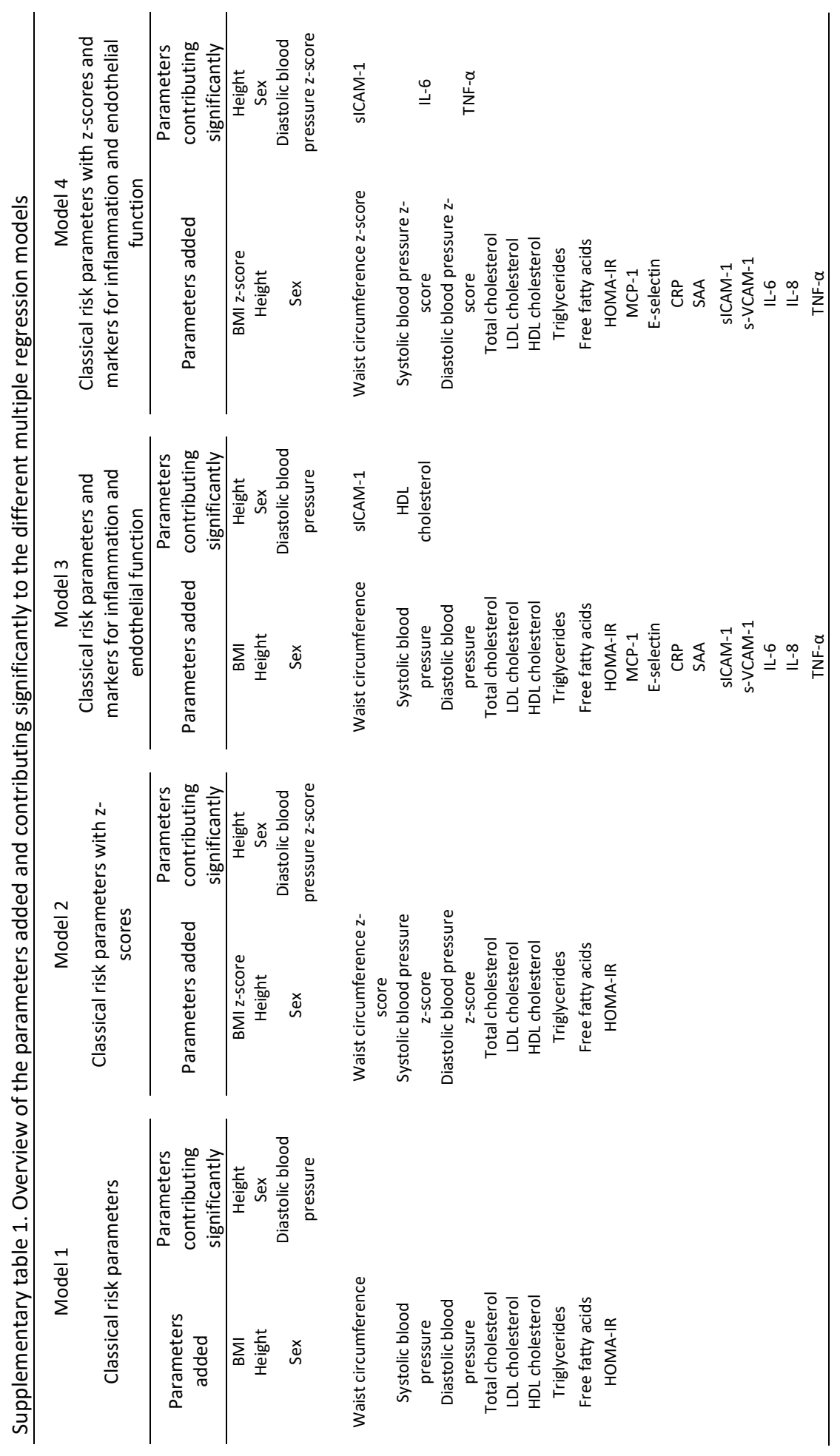


Supplementary table 2. Augmentation and height index across puberty status, stratified for sex

\begin{tabular}{|c|c|c|c|c|}
\hline & Pre-pubertal & Pubertal & Post-pubertal & $\mathrm{p}$-value \\
\hline$n(M / F)$ & $24 / 24$ & $8 / 22$ & $21 / 45$ & \\
\hline \multicolumn{5}{|l|}{ Alx@75,\% } \\
\hline Boys & $21.0(10.8 ; 28.8)$ & $16.5(7.3 ; 28.8)$ & $3.0(-2.5 ; 9.5)$ & $<0.001$ \\
\hline Girls & $27.5(23.3 ; 35.0)$ & $24.0(9.8 ; 32.5)$ & $21.0(13.0 ; 26.0)$ & 0.007 \\
\hline \multicolumn{5}{|l|}{ Height, $\mathrm{cm}$} \\
\hline Boys & $140.6 \pm 11.1$ & $160.3 \pm 9.2$ & $176.5 \pm 8.5$ & $<0.001$ \\
\hline Girls & $135.2 \pm 12.2$ & $153.7 \pm 9.2$ & $163.7 \pm 7.6$ & $<0.001$ \\
\hline
\end{tabular}

Data presented as median (Q1-Q3). $P$-value presented are for the difference in Alx between puberty groups.

Supplementary table 3. Alx@75x values across IL-6 and hs-CRP tertiles, stratified for sex

\begin{tabular}{rcccc}
\hline & $1^{\text {st }}$ tertile & $2^{\text {nd }}$ tertile & $3^{\text {rd }}$ tertile & $p$-value \\
\hline$I L-6, p g / m L$ & & & \\
Girls & $21.0(12.3 ; 27.0)$ & $23.0(15.0 ; 31.5)$ & $24.0(18.0 ; 29.0)$ & $\mathrm{p}=0.354$ \\
Boys & $13.0(4.8 ; 18.3)$ & $13.5(3.8 ; 22.5)$ & $16.5(6.3 ; 25.5)$ & $\mathrm{p}=0.691$ \\
Girls & $23.0(15.0 ; 28.3)$ & $24.0(15.5 ; 30.0)$ & $21.0(13.8 ; 32.0)$ & $\mathrm{p}=0.857$ \\
Boys & $13.0(3.00 ; 19.5)$ & $8.0(4.0 ; 21.0)$ & $19.0(10.0 ; 24.8)$ & $\mathrm{p}=0.238$ \\
\hline
\end{tabular}

Data presented as median (Q1-Q3). P-value presented are for the difference in Alx between the tertiles; IL-6 = interleukin 6; CRP = C-reactive protein. 
Chapter 4 


\section{Chapter 5}

A narrative review of factors associated with
development and progression of non-alcoholic
fatty liver disease

Annemiek De Nooijer Anita C.E. Vreugdenhil

Kylie Karnebeek Peter van Hasselt Sabine Fuchs 


\section{Abstract}

\section{Background}

With the obesity pandemic, non-alcoholic fatty liver disease (NAFLD) has become the most prevalent liver disease. NAFLD can progress to non-alcoholic steatohepatitis (NASH), a potential cause of liver failure. It remains difficult to identify patients at risk for NASH, despite evolving insights in contributing factors, including genetic variance, hormones, adipokines, diet and body-fat distribution. We aimed to present a broad perspective on these risk factors associated with NAFLD development and progression with a focus on their contribution in different age groups and susceptible high-risk populations, hereby giving insight in the pathophysiology of NAFLD.

\section{Methods}

Literature was searched for relevant articles on the pathophysiology of NAFLD in different age-groups.

\section{Results}

Our review underscores large contributions of diet, with particularly fructose promoting NASH development; and sex hormones, with oestrogens exerting protective effects and androgens negatively influencing NAFLD development. Genetic variation in corresponding pathways might further determine NAFLD progression.

\section{Conclusions}

Changes throughout the transition from childhood to adulthood show that variations in diet, hormone levels and metabolism are related to NAFLD progression. The human body uses different strategies to handle excessive nutrients, but each comes at a price. When corresponding pathways are strained by hormonal or genetic factors, NASH or other symptoms of the metabolic syndrome ensue. Potentially, stratification based on sex, body-fat distribution, diet, lifestyle, microbiome, adipokines, sex hormones, blood concentrations of liver enzymes, liver histology and genetic predisposition might help to identify patients at increased risk of NASH. 


\section{Highlights}

1. Non-alcoholic fatty liver disease (NAFLD) is the most prevalent liver disease, but factors contributing to progression to severe non-alcoholic steatohepatitis (NASH) are insufficiently known for targeted prevention or intervention strategies.

2. Exploration of NAFLD development in various age groups, from childhood to late adulthood gives insight in specific disease contributing factors.

3. Hormonal factors (androgens opposed to oestrogens) and dietary fructose appear most harmful in promoting NAFLD progression and the latter represents an easy target for intervention.

4. The human body has different ways to handle excessive nutrients, but eventually each way leads to a burden such as NAFLD or cardiovascular disease.

5. Stratification based on sex, body-fat distribution, diet, lifestyle, microbiome, adipokines, sex hormones, blood concentrations of liver enzymes, liver histology and genetic predisposition might help to identify patients at increased risk to develop NASH, but current genetic screening possibilities might reveal novel or more specific targets for prevention or intervention strategies. 


\section{Introduction}

Non-alcoholic fatty liver disease (NAFLD) is the most common liver disorder in the developed world (1). The disease has a varying severity, ranging from reversible hepatic steatosis, characterized by excessive accumulation of triglycerides (TG) in the liver $(2,3)$, to non-alcoholic steatohepatitis (NASH), consisting of macrovesicular steatosis, lobular inflammation and hepatocyte injury $(3,4)$. NASH can progress to fibrosis, cirrhosis, liver failure and hepatocellular carcinoma. Globally, $25 \%$ of adults have hepatic steatosis. Obesity and type 2 diabetes mellitus significantly raise this risk; NAFLD rates of $70 \%$ and higher have been reported in these populations. NASH occurs in 1,5 to $6,45 \%$ of adults (5). Furthermore, $8 \%$ of children in the developed world and $34 \%$ of obese children suffer from hepatic steatosis, which has become the most common liver abnormality in children (6). Approximately $23 \%$ of children with steatosis have NASH (7).

The heritability of NAFLD approaches $40 \%(3,8)$ and mutations in certain genes associated with lipid metabolism are known to cause NAFLD in patients with and without obesity (3). However, the determinants and mechanisms driving progression of simple steatosis to more severe forms of NASH, sometimes resulting in liver fibrosis already in childhood, are incompletely understood.

To make the next step in clinical care for patients with NAFLD, a deep understanding of the pathogenesis of NAFLD and modifiers determining progression of liver disease is crucial. We set out to deepen this understanding by studying patients with severe forms of NAFLD and search for specific disease modifying factors. We considered children with NASH of particular interest since they have an early disease onset and are subject to large biological fluctuations in hormones, metabolism, insulin resistance (IR) and lifestyle. Likewise, severe phenotypes of NAFLD are seen with specific genetic variants providing insight in the contribution of affected pathways to NASH development (9). Understanding the factors that predispose to rapid disease progression can help to identify patients at increased risk for developing severe liver pathology and develop novel prevention and treatment strategies. In this narrative review we will discuss the pathogenesis of NAFLD, elaborating on the relationship with insulin resistance (IR) and lipid metabolism. Subsequently, we will analyse important factors in progression to NASH such as dysbiosis and TLR4 activation, the role of adipokines and how lipotoxicity is translated in oxidative and endoplasmic reticulum stress, leading to apoptosis. We will elaborate on risk factors that are subject to change during the transition from childhood to adulthood and on genetic variants that predispose to NAFLD and NASH. 


\section{Methods}

PubMed electronic database from 2003 to 2018 was searched for relevant publications using the following terms: "NAFLD" OR "Non-alcoholic fatty liver disease" OR "NASH" OR "Non-alcoholic steatohepatitis" AND "pathogenesis" OR "mechanism" OR "pathology". This review focuses on risk factors in the transition from childhood to adulthood, for which we repeated the previous search combined with the following terms: AND "Child" OR "Childhood" OR "Children" Or "Adolescent" OR "Puberty" OR "Pubertal" OR "Paediatric" OR "Pediatric". The inclusion criteria were (i) Peer-reviewed academic journals published in English (ii) research that focused on NAFLD development and factors associated with NAFLD development (iii) articles with accessible abstracts and full text. Articles were read and assessed for relevance. Hand searches of the references of retrieved literature was done. Backward snowballing was used to complement the database search.

\section{Pathogenesis of steatosis and insulin resistance}

Central to hepatic steatosis are TG accumulation and IR. Hepatocytes accumulate TGs through fatty acid (FA) acquisition from dietary fat, adipose tissue lipolysis, and hepatic lipogenesis. They clear TGs through FA $\beta$-oxidation and TG export as very low-density lipoprotein (VLDL) via the endoplasmic reticulum (ER) (Figure $1 A)(3,10,11)$. Insulin influences these processes by inducing lipogenesis and antagonizing tissue lipolysis.

\section{Obesity-induced systemic IR and FA release}

Obese patients become less responsive to insulin, which is often attributed to chronic inflammation of adipose tissue. In obesity, storage of excessive TGs leads to hypertrophic adipose tissue, which enhances production of pro-inflammatory cytokines TNF- $\alpha$, IL-1, IL6 , and monocyte chemoattractant (MCP-1) (11). These pro-inflammatory factors antagonize the lipogenic effects of insulin, thereby contributing to systemic IR, which increases adipose tissue lipolysis. Together, this leads to increased hepatic FA concentrations $^{11}$. Furthermore, MCP-1 attracts macrophages, creating low-grade inflammation. These macrophages and other resident cells subsequently produce even higher levels of inflammatory cytokines, which promote leptin production, and reduce adiponectin production $(10,11)$ and affect IR (12).

Adipose tissue inflammation, IR and increased FA influx in the liver stimulate each other, creating a positive feedback loop. More than half of hepatic fat is derived from lipolysis 
from adipose tissue (3) and this correlates with total fat mass in humans, resulting in increased FA release in obese patients. Normally, insulin inhibits adipose tissue lipolysis, but this is impaired in patients with systemic IR, resulting in elevated efflux of FAs from adipose tissue $(11,13)$. In the liver, the hyperinsulinemia associated with IR inhibits $\beta$ oxidation and causes an upregulation of transcription factor SREBP-1c leading to enhanced lipogenesis $(3,11,13)$. These combined effects lead to hepatic steatosis $(11,13)$. Within this positive feedback loop, it is unclear whether IR precedes or follows steatosis (3).

Body fat distribution is important, as visceral adipose tissue produces more proinflammatory cytokines, pro-hyperglycaemic factors and less adiponectin than subcutaneous adipose tissue (15). Hence, IR and NAFLD have a stronger correlation with visceral adiposity than with BMI (14). Also, IR seems to decrease with higher skeletal muscle mass, which can be attributed to insulin-mediated glucose utilization. A 7-year follow-up of patients with NAFLD showed a correlation between increased skeletal muscle mass and improvement or prevention of NAFLD, which might contribute to a stratification for at-risk disease-susceptible individuals (15).

\section{FA influx in the liver and hepatic IR}

In the liver, FA are metabolized through $\beta$-oxidation, exported as VLDL or stored as TGs (Figure 1A). Elevated FA concentrations lead to accumulation of intermediate products such as diacylglycerol (DAG). Cytosolic DAG enhances translocation of protein kinase $C \varepsilon$ $(\mathrm{PKC} \varepsilon)$ to the cell membrane, where it inhibits insulin signalling via reduced phosphorylation of the insulin receptor substrate 2 (IRS-2)(16). This in turn suppresses glycogen synthase activity, leading to decreased glycogen synthesis. In parallel, reduced IRS-2 activity causes translocation of Forkhead box protein $\mathrm{O} 1$ to the nucleus, where it enhances expression of various gluconeogenic enzymes (16). Cytosolic DAG thus inhibits IRS-2, which leads to hepatic IR and enhanced gluconeogenesis and inhibited glycogen synthesis (17).

\section{Pathogenesis of NASH}

Elevated hepatic FA concentrations increase the susceptibility of the liver to injury and are seen as the first hit. Multiple hits including IR, nutritional factors, adipokines, gut microbiota and genetic factors are now thought to cause the liver to progress from steatosis to NASH (Figure 1B)(10,13,18). 

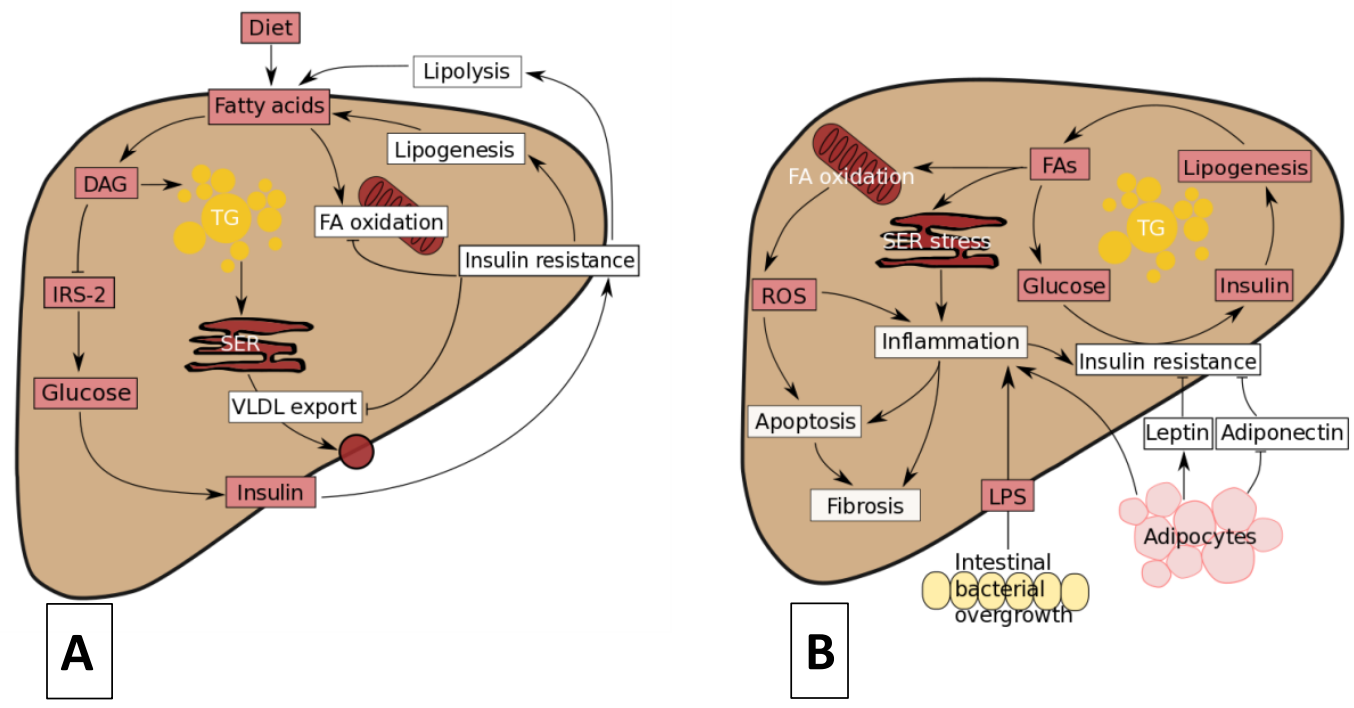

Figure 1: Interpretation of the current literature on the pathogenesis of steatosis (A) and factors contributing to the progression to NASH (B)

$F A=$ fatty acid; $S E R=$ smooth endoplasmic reticulum; $V L D L=$ very low-density lipoprotein; $D A G=$ diacylglycerol; $T G$ = triglyceride; $I R S-2$ = insulin receptor substrate $2, R O S=$ Reactive oxygen species, LPS = lipopolysaccharides.

A. Increased FA influx from lipolysis in adipose tissue, diet, and lipogenesis causes increased IR via DAG and decreased phosphorylation of IRS-2, which leads to elevated glucose via enhanced gluconeogenesis and reduced glycogen synthesis. Elevated glucose levels trigger more insulin release. Insulin resistance stimulates lipolysis of adipose tissue and de novo lipogenesis, while it decreases VLDL export, further contributing to increased FA concentrations and steatosis.

B. Elevated $\beta$-oxidation of FAs generates ROS. FAs are incorporated in the ER membrane, leading to the unfolded protein response. Both pathways cause hepatocyte apoptosis and contribute to inflammation. Inflammation is further triggered by intestinal bacterial overgrowth and adipose tissue inflammation. Inflammatory cytokines increase IR. Adipose tissue ameliorates insulin sensitivity via increased production of leptin and adiponectin. Adiponectin production is decreased in NASH. Although leptin production is increased in NAFLD, this does not lead to lower insulin concentrations, suggesting leptin resistance. With this resistance, the protective effect of leptin against hepatic steatosis is lost.

\section{Inflammation}

Pro-inflammatory cytokines play a crucial role in the progression from steatosis to NASH (3). First, it has been demonstrated in rodents that specific cytokines can elicit the same response in liver tissue as seen in $\mathrm{NASH}$, including neutrophil chemotaxis, hepatocyte apoptosis/necrosis, Mallory body formation and stellate cell activation (11). Second, enhanced expression of TNF- $\alpha$ and IL- 6 in adipose tissue was detected in obese subjects before liver inflammation was present (19), suggesting that adipose tissue mediated cytokine release precedes, and might lead to liver inflammation. Third, inhibition of TNF- $\alpha$ led to amelioration of IR and histological parameters of NASH in an obese mouse model on a high-fat diet (20). 
Cytokines lead to NASH development through activation of the nuclear factor $\mathrm{\kappa B}$ (NF-kB) and $\mathrm{C}$-Jun $\mathrm{NH}_{2}$-terminal kinase (JNK) pathway. FAs, particularly saturated FA, were found to activate the toll-like receptor 4 (TLR4), activating both the NF-KB and JNK pathway $(21,22)$. In the liver TLR4 is expressed by hepatocytes, Kupffer cells and stellate cells. Upon phagocytosis of cholesterol, Kupffer cells are activated and upregulate TLR4 expression (23). Activated Kupffer cells produce transforming growth factor $\beta$ (TGF- $\beta$ ), which triggers the fibrogenic state of stellate cells (24).

The importance of TLR4 signalling in the pathogenesis of NASH is illustrated by TLR4 mutant mice, that neither develop extreme adiposity and IR in response to a high saturated fat diet nor NASH in response to a methionine/choline-deficient diet $(25,26)$. Thus, inflammation through TLR4 receptor activation appears to be a major factor in murine models in the progression from steatosis to NASH. In humans these mechanisms still require confirmation.

\section{Adipokines}

In addition to being a major source of FAs and cytokines (IL- 6 and TNF- $\alpha$ ), adipose tissue releases adipokines, such as leptin and adiponectin (10). Leptin and adiponectin enhance hepatic insulin sensitivity (12) and appear to be important factors in the pathogenesis of NASH.

Under non-obese conditions, leptin protects against NAFLD. Leptin acts centrally to decrease food intake and has anti-hyperglycaemic effects (27). In response to lower glucose concentrations, insulin concentrations decrease, reducing hepatic lipogenesis and increasing lipolysis. Similarly, in the liver leptin stimulates FA $\beta$-oxidation and suppresses lipogenesis in vitro, decreasing fat accumulation and lipoapoptosis $(10,27)$. Leptin is markedly increased in obesity and hepatic steatosis, and even more in NASH (27). High leptin levels do not decrease insulin concentrations or IR in obese humans. Potentially, the protective effects of leptin are lost in obesity due to excessive leptin exposure and leptin resistance. This might be caused by suppressor of cytokine 3 (SOCS-3) which is activated by leptin and inhibits leptin signalling when overexpressed. SOCS-3 expression is also stimulated by insulin and SOCS-3 overexpression leads to IR, whereas inhibition of SOCS-3 ameliorates insulin sensitivity and hepatic steatosis (27). In obesity, leptin enhances proinflammatory signalling cascades via activation of NF-KB (28). Moreover, prolonged hyperleptinemia may lead to fibrosis via activated stellate cells (27). Activated stellate cells, Kupffer cells and sinusoidal cells all express the leptin receptor during fibrosis development $(27,28)$. Leptin facilitates stellate cell proliferation. Once activated, stellate cells contribute to leptin expression, creating a positive feedback loop (Figure 2). Hence, in 
normal non-obese conditions, leptin protects against hepatic steatosis by decreasing lipid intake, carbohydrate intake and synthesis, and increasing lipid metabolism. When these signals are ignored, leptin is excessively secreted, putatively leading to leptin resistance and hepatic inflammation and fibrosis (Figure 2).

Adiponectin promotes insulin sensitivity and reduces inflammation, thereby protecting against NAFLD progression. Total body fat is inversely correlated with adiponectin, and adiponectin blood concentrations are lower in patients with NASH than with steatosis $(19,28)$. Adiponectin increases FA $\beta$-oxidation and decreases lipogenesis, thereby reducing FA concentrations $(18,28)$. Adiponectin upregulates IRS-2 in an obese mouse model, thereby promoting insulin sensitivity (29). Insulin sensitivity is further increased by adiponectin via antagonizing TNF- $\alpha$ and IL-6 effects, and enhancing secretion of antiinflammatory cytokines IL-10 and IL-1 receptor antagonists in human leukocytes in vitro (30). Adiponectin downregulates TLR4-induced NF-KB activation and leads to reduced TNF$\alpha$ production in rat Kupffer cells in vitro and in mice upon exposure to lipopolysaccharides $(\text { LPS })^{31}$. Finally, adiponectin has been shown to inhibit stellate cell proliferation in vitro, possibly via inhibition of ROS production (30)(Figure 2). Summarizing, adiponectin promotes insulin sensitivity, protects from liver inflammation and limits fibrosis (Figure 2). Decreased adiponectin levels might predispose NAFLD patients to progress to NASH. 


\section{A}

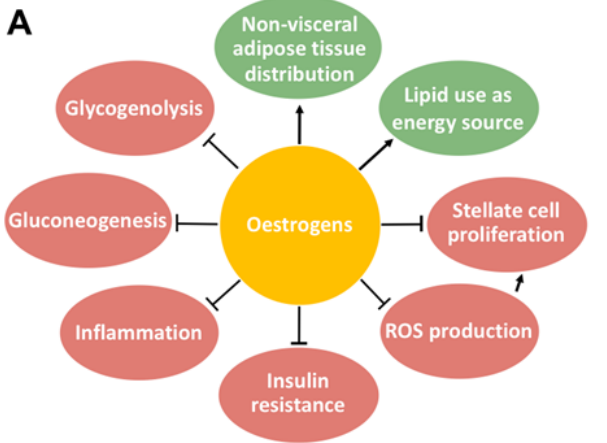

C

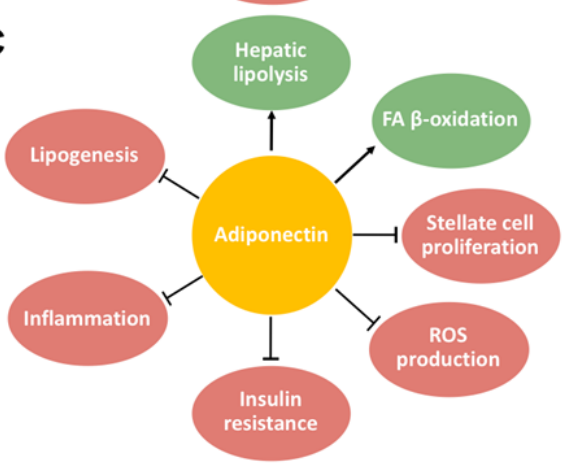

B

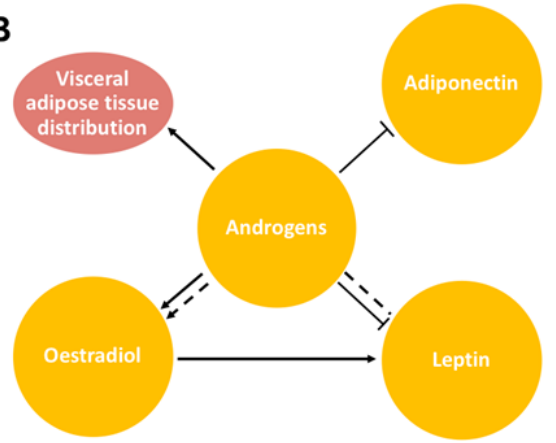

D

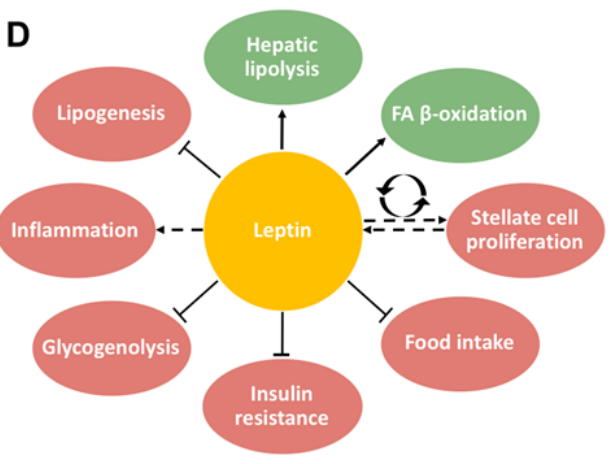

Figure 2: Schematic representation of the effects related to NAFLD/NASH of oestrogens, androgens, adiponectin and leptin

A. Combined effects of oestrogens reduce the chance of progression to NASH. B. Androgens promote visceral adipose tissue distribution and inhibit production of adipokines, thereby increasing the risk of progression to NASH. In obesity, androgen conversion to oestrogen is increased, but inhibition of leptin production by androgens is lost. C. Adiponectin inhibits several risk factors for progression to NASH and promotes hepatic lipolysis and FA $\beta$-oxidation. D. Leptin has similar effects to adiponectin, but these are partly lost in obesity, putatively related to leptin resistance. Moreover, high leptin levels trigger stellate cell activation and stimulate stellate cell proliferation, instigating a positive feedback loop with activated stellate cells producing leptin, thereby contributing to progression to NASH.

-: effects under normal conditions; ---: pronounced or changed effects in obesity; T: stimulation; $\perp$ : inhibition

\section{Dysbiosis}

The microbiome composition of patients with obesity or NAFLD is different from controls $(23,38)$ and small intestinal overgrowth is more common in NAFLD (39). A different microbiome composition may elevate absorption of calories and increased FA uptake in adipocytes and hepatocytes, contributing to $\operatorname{NAFLD}(38,40,41)$. Small intestinal bacterial overgrowth correlates with severity of steatosis (39). Bacterial overgrowth and the NAFLD microbiome composition are associated with increased gut permeability, caused by 
disrupted intercellular tight junctions (23). This leads to leakage of LPS, derived from gram-negative bacteria in the gut into the circulation. LPS have pro-inflammatory effects on hepatocytes, Kupffer cells, and stellate cells. Activation of TLR4 by LPS triggers proinflammatory gene expression, formation of inflammasomes, and generation of ROS via NADPH oxidase 2 , which lead to hepatic injury $(22,23,37)$. Adults with a high-fat intake (42) and children with NAFLD (37) both showed elevated levels of circulating LPS. Highfructose intake has been linked to increased gut permeability in mice via translocation of LPS and activation of TLR4 (43). Additionally, NAFLD patients have higher serum ethanol levels, which is attributed to more fermenting bacteria in their microbiome (44). This increases translocation of endotoxins, TLR4 activation, generation of ROS and TNF- $\alpha$ (44). Concurrently, probiotic treatment of paediatric NAFLD leads to an improved lipid profile and decreased hepatic steatosis (45-47). These data suggest that high-fat and highfructose diets contribute to altered gut microbiome and translocation of LPS into the circulation, which stimulates hepatic inflammation via TLR4.

\section{Paediatric NAFLD}

NAFLD already occurs in early childhood (14), but is more common in adolescents (8). Insight in paediatric NASH can be considered of specific relevance to identify factors affecting fast progression of NAFLD. In addition, paediatric patients offer the opportunity to evaluate effects of lifestyle, growth, anthropometry and hormonal factors on the pathogenesis of NAFLD, since these factors all highly fluctuate during the transition from childhood to adulthood.

\section{Histology}

Paediatric NASH differs in histological characteristics from adult NASH. Whereas adult NASH is characterized by perisinusoidal changes including steatosis, lobular inflammation, hepatocellular ballooning and fibrosis in the absence of portal changes (defined as type 1) (4), paediatric NASH predominantly includes portal steatosis, inflammation, and fibrosis (defined as type 2 ) $(48,49)$, or an overlap between both types. The portal pathology is most striking in boys, severe metabolic syndrome and severe obesity $(1,7,50,51)$. The portal area is exposed to blood first, receiving highest concentrations of nutrients, oxygen, hormones and inflammatory or toxic substances, and harbours the highest concentration of Kupffer cells. Portal hepatocytes are specialized in oxidative liver functions such as gluconeogenesis, FA $\beta$-oxidation and cholesterol synthesis (52). One might thus 
hypothesize that increased oxidative stress or high concentrations of harmful nutrients such as fructose result in portal injury and might accelerate progression from steatosis to $\mathrm{NASH}$ in children. This is supported by the finding of increased steatosis in the portal area in rats fed with both sucrose and fructose-enriched diets Paediatric NASH differs in histological characteristics from adult NASH. Whereas adult NASH is characterized by perisinusoidal changes including steatosis, lobular inflammation, hepatocellular ballooning and fibrosis in the absence of portal changes (defined as type 1)(4), paediatric NASH predominantly includes portal steatosis, inflammation, and fibrosis (defined as type $2)(48,49)$, or an overlap between both types. The portal pathology is most striking in boys, severe metabolic syndrome and severe obesity $(1,7,50,51)$. The portal area is exposed to blood first, receiving highest concentrations of nutrients, oxygen, hormones and inflammatory or toxic substances, and harbours the highest concentration of Kupffer cells. Portal hepatocytes are specialized in oxidative liver functions such as gluconeogenesis, FA $\beta$-oxidation and cholesterol synthesis (52). One might thus hypothesize that increased oxidative stress or high concentrations of harmful nutrients such as fructose result in portal injury and might accelerate progression from steatosis to NASH in children. Similarly, Nobili et al. recently showed that high-fructose intake in children correlated with increased portal steatosis, inflammation and hypothesis (53).

\section{Lifestyle and diet}

During childhood children change their lifestyle habits, which influence NAFLD progression in a gender dependent manner. During adolescence physical activity decreases, while sugar and fat intake increase (54). Girls are less active than boys and already have reduced levels of physical activity before the onset of puberty $(55,56)$, which does not correspond with the observation of lower NASH prevalence in girls. The high NASH prevalence in boys correlates with the high-carbohydrate intake in boys (56). High-carbohydrate intake in humans has been linked to increased IR and portal FA concentrations, upregulation of lipogenesis and inhibition of $\beta$-oxidation, all contributing to increased FA load and steatosis (57). More specifically, fructose intake has emerged as a determinant in NAFLD (58). The average yearly consumption of fructose has significantly increased over the years (59). Fructose consumption is higher in children from nine to eighteen years compared to adults (60) and twice to threefold higher in NAFLD patients compared to controls $(58,61,62)$. While glucose mainly serves as a substrate for glycogenesis, fructose mainly serves as a substrate for lipogenesis (63). High fructose instead of glucose intake was found to increase dyslipidaemia, intrahepatic TG accumulation, IR, hepatic inflammation and fibrosis in humans $(10,64,65)$. High-fructose diet elicited inflammatory changes in the 
JNK pathway (66) and enhanced SOCS-3 expression inducing leptin resistance in rats (59). High-fructose diet was further found to induce low-grade endotoxemia via enhanced intestinal permeability, thereby predisposing to $\operatorname{NASH}(43,61)$. Overall, the effects of fructose on NAFLD are clearly illustrated by the decrease in liver transaminases induced by a low fructose and low glycaemic index diet without caloric restriction in children with NAFLD (67). Similarly, obese children showed reduced hepatic fat, visceral fat, lipogenesis and IR already after nine days of fructose restriction (63).

\section{Growth (anabolism)}

Insulin sensitivity fluctuates with sex and age $(66,68)$ (Figure 3 ). At the beginning of puberty, IR is similar in both sexes and gradually rises during puberty $(66,68)$. Growth hormone (GH) secretion at night leads to increased IR (69). To compensate for IR, insulin secretion increases two to threefold during puberty (69).

$\mathrm{GH}$ and its downstream primary mediator insulin-like growth factor 1 (IGF-1) play an important role in the regulation of lipid and glucose metabolism. GH stimulates adipose tissue lipolysis, increasing the hepatic influx of FAs. GH also enhances hepatic lipogenesis through activation of SREBP-1c (70). GH signalling stimulates hepatic TG secretion via increased VLDL export and enhances FA oxidation (70,71). Overall, GH lowers fasting free FAs (69). GH stimulates hepatic glucose production, and triggers IR specifically in adipose tissue and muscle, leading to hyperinsulinemia. Hyperinsulinemia synergizes with $\mathrm{GH}$ and IGF-1 to stimulate protein anabolism supporting growth.

The increase in GH, IGF-1 and insulin concentrations during adolescence ensure anabolism and thereby protect against steatosis. Circulating GH and IGF-1 levels are lower in NAFLD patients than in controls $(72,73)$. Patients with $\mathrm{GH}$ deficiency have an increased NAFLD prevalence (72). Moreover, GH secretion is reduced in obesity (74), without a proportional decrease in IGF-1 concentrations (70), maintaining a negative feedback loop for GH. This consequently enhances NAFLD progression. GH replacement therapy in a patient with $\mathrm{GH}$ deficiency reversed NASH $(70,74)$. Furthermore, treatment with $\mathrm{GH}$ reduced visceral adiposity and liver fat (75). All these studies support the anabolic role for GH and IGF-1, protecting against IR and hepatic steatosis during puberty $(69,70)$.

\section{Hormonal sex differences during puberty}

The role of hormonal influences in the development of NASH is implied by studies showing that NAFLD is more common in boys than girls (7), nearly reaching a ratio of 2:1 in obese children (6) and further diverges during adolescence, increasing in boys and decreasing in 
girls (76). In obese children, liver steatosis increases from $40 \%$ to $51 \%$ during puberty in boys, while it decreases from $17 \%$ to $12 \%$ in girls (reaching $25 \%$ mid-puberty; Figure 4)(76). During puberty, oestrogen secretion increases steadily in girls and only slightly in boys (Figure 3). Oestrogens reduce gluconeogenesis and glycogenolysis (77). Consequently, human subjects with deficient oestrogen synthesis exhibit increased IR, which can be resolved by oestrogen supplementation (77). Oestrogens have antioxidant properties, as they inhibited ROS generation, lipid peroxide levels, hepatic inflammation, stellate cell proliferation and activation, apoptosis and fibrosis in rats (28)(Figure 2). Oestrogens stimulate the use of lipids as energy source and promote non-visceral adipose tissue distribution (78)(Figure 2).

Leptin levels also show a sex-dependent pattern: in girls, leptin concentrations increase steadily during puberty, while decreasing from midpuberty onwards in boys (Figure 3 ). Androgens inhibit leptin production, but this association is lost in obesity as leptin levels increase (79)(Figure 2 and Figure 4). This might be explained by enhanced conversion of androgens to oestrogens in obesity (77). Moreover, oestrogens are thought to increase the sensitivity to central leptin (78). In obese boys and girls, leptin concentrations are high throughout puberty, with a tendency to increase in puberty in girls and decrease in boys (Figure 4), potentially resulting in leptin resistance via increased SOCS-3 expression (27). Leptin appears as an independent determinant of pubertal IR (68).

At the beginning of puberty, adiponectin levels are similar in girls and boys and decrease significantly during puberty (68)(Figure 3). In girls, the decrease in adiponectin levels corresponds with the pubertal increase in adipose tissue. The more severe decrease of adiponectin levels in boys can be explained by increasing androgen levels since adiponectin concentrations are inversely correlated with androgen concentrations $(68,80)$. In overweight or obese children, adiponectin levels correlate inversely with IR (68)(Figure 4).

The prevalence of hepatic steatosis is twofold higher in obese boys than in obese girls. This cannot be explained by IR differences (Figure 4). The higher leptin concentrations and sensitivity, and slightly higher adiponectin concentrations (68) in girls from midpuberty onwards might contribute to this difference. These differences can be enhanced by the sex hormones and exogenous factors such as diet. Female sex hormones contribute to a protective fat distribution for NAFLD progression, increased leptin and reduced inflammation and oxidative stress. In men, androgens predispose to accumulation of visceral adipose tissue, low leptin and adiponectin concentrations and no direct NAFLD protective effects. 

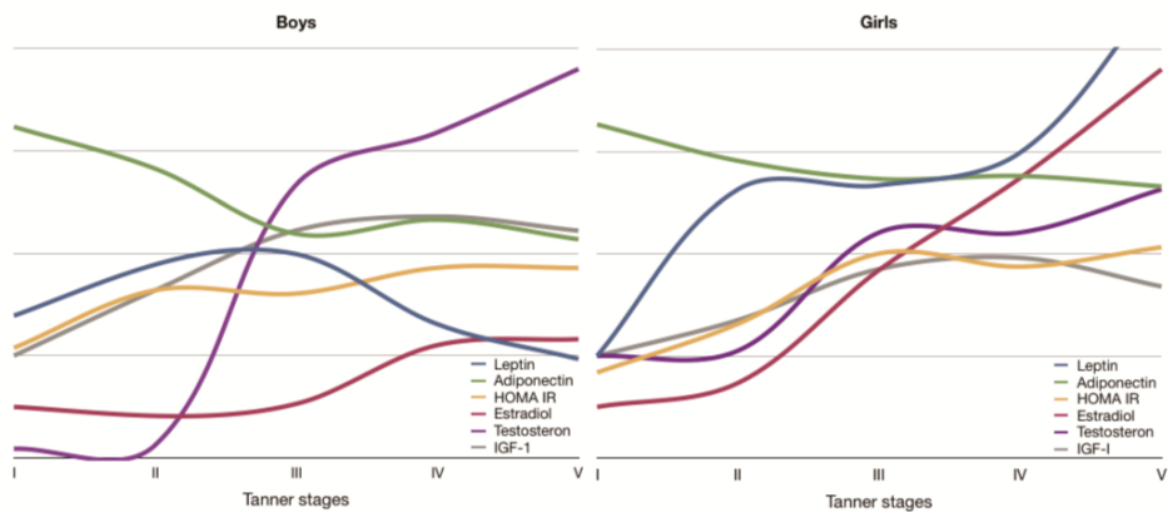

Figure 3. Fluctuations of potentially relevant factors in NAFLD pathogenesis in boys and girls during puberty.

All trends are based on the general population. Leptin trends are derived from Xu et al., Horlick et al., and Ellis et al. $(68,99,100)$; adiponectin from Xu et al., Martos-Moreno et al., Böttner et al. and Andersen et al. $(68,80,101,102)$; insulin resistance (HOMA IR) from Xu et al.(68) and calculated by homeostasis model assessment of insulin resistance; oestradiol from Sehested et al. (103), Konforte et al. (104) and LabCorp; testosterone from Konforte et al. (104); and IGF-1 from reference values from Mayo medical laboratories. Distributions were linearly transformed to display relative differences during pubertal stages for individual sexes.

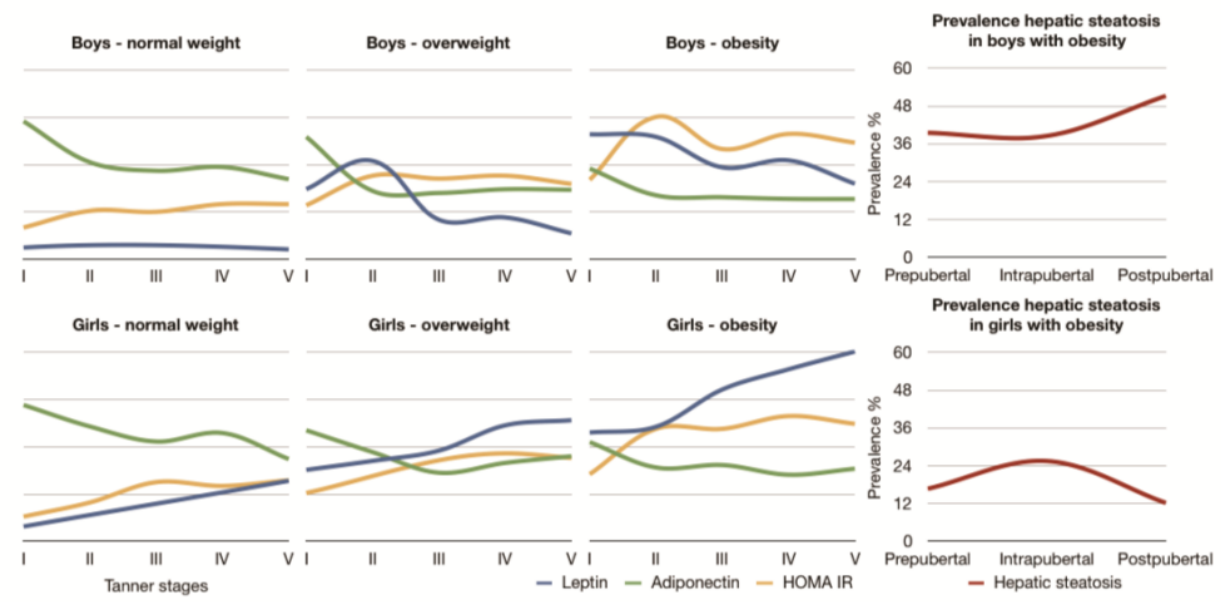

Figure 4. Leptin, adiponectin and insulin resistance trends in boys and girls according to weight status and prevalence of hepatic steatosis in boys and girls with obesity.

Leptin, adiponectin and insulin resistance trends are derived from Xu et al. (68), with HOMA IR calculated by homeostasis model assessment of insulin resistance. Prevalence of hepatic steatosis, determined by ultrasound examination, is derived from Denzer et al. (76). Distributions were linearly transformed to display relative differences during pubertal stages for individual sexes. 


\section{Genetic variants causing NAFLD/NASH}

NAFLD development and progression is thought to result of a complex interplay between environmental, metabolic and genetic risk factors. Genome-wide-association studies (GWAS) have revealed several overlapping genetic risk loci for NAFLD development in adults, children and animal models (81). The I148M-variant (rs738409) in the patatin-like phospholipase domain containing 3 (PNPLA3) gene, is a common single nucleotide polymorphism (SNP) that is associated with an increased prevalence of hepatic steatosis, increased ALT concentrations, severe inflammation and fibrosis (82). The PNPLA3 protein has lipase activity towards TGs and is highly expressed in hepatocytes and stellate cells (83). Impaired TG hydrolysis in hepatocytes affects lipid droplet size (84) and leads to accumulation of hepatic TGs (85) in people carrying the I148M variant without affecting IR (86). Increased liver fat content was found in homozygote carriers of the PNPLA3 variant already under the age of ten years (87). Rapid NAFLD progression in patients with PNPLA3 SNPs underscores the importance of abnormal lipolysis in hepatocytes in NAFLD progression. In hepatic stellate cells the I148M variant leads to reduced hydrolysis of retinol esters, leading to lower free retinol levels and elevated intracellular retinol retention in stellate cells (83). This activates hepatic stellate cells and eventually predisposes to fibrosis, but pathway connecting the $1148 \mathrm{M}$ variant to inflammation and fibrogenesis remain unclear. Additionally, the PNPLA3 I148M variant increases susceptibility for hepatocellular carcinoma (HCC)(88).

Another strong genetic predictor for NAFLD in children and adults is the glucokinase regulator (GCKR) SNP (rs1260326), that inhibits GCKR's response to fructose-6-phosphate and associates with liver fat content in $\operatorname{NAFLD}(89,90)$.

Another common SNP that is associated with NAFLD is the transmembrane 6 superfamily member 2 (TM6SF2) E167K variant (rs58542926). This variant decreases TM6SF2 function, leading to reduced VLDL secretion (91). Consequently, hepatic TG content increases and circulating lipid concentrations decrease, leading to an improved overall cardiovascular risk profile, but increased risk of NASH $(92,93)$. The association between increased hepatic steatosis in patients with the TM6SF2 SNP and advanced liver disease has been found both in adults and children (94). The association of this gene with NAFLD suggests a significant role for VLDL export in preventing NASH.

Other candidate genes associated with NAFLD have been proposed, including a variant (rs641738) in the membrane bound O-acyltransferase domain containing 7transmembrane channel-like 4 (MBOAT7) gene, which is associated with NAFLD in adults. Children carrying the minor allele of the MBOAT7 gene have higher ALT plasma levels compared to non- carriers (95). 
Genetic modifiers of oxidative stress, inflammatory response, IR, lipid metabolism, fibrogenesis and satiety all have been suggested to influence the pathology of NAFLD. The role of most of these genes is less established and requires further elucidation particularly in paediatric NAFLD. So far, potential NAFLD modifying genes were not reported to differ between children and adults. Moreover, it is likely that the risk of NAFLD progression is determined by the combined effect of risk genes (96).

\section{Conclusion}

We live in an era in which many persons have an unhealthy lifestyle including excess of food and insufficient physical activity. The harmful impact of this unhealthy lifestyle on disease development is becoming more and more apparent. Research on the contributing factors and mechanisms enabling identification of people at a particularly high risk for development of NAFLD is urgently needed. From the current literature it can be concluded that diet, lifestyle, fat distribution and microbiome are partially influenceable factors that can predispose to NASH, whereas gender, sex hormones and genetic predisposition are also risk factors, but cannot be influenced. All these factors, together with blood concentrations of liver enzymes and liver histology could potentially be used to stratify individuals with NAFLD into a low or high risk of progression to NASH.

Many of these factors that determine NAFLD progression, including lifestyle, body-fat distribution, IR, adipokines and hormone concentrations are subject to enormous changes during the transition from childhood to adulthood. From studies on paediatric NAFLD, we deduce that oestrogens exert protective effects through a favourable fat composition. With rising oestrogen levels during puberty, leptin concentrations also increase, while androgens inhibit leptin secretion. The prepubertal gender differences in NAFLD underscore the involvement of other factors as well. Dietary intake seems an important contributing factor. The specific portal histological abnormalities in children suggest an important role for oxidative stress and toxic substances, which appear related to highfructose intake. Awareness of the damaging effects of fructose is necessary to prevent further increase in NAFLD incidence and progression, especially because this might guide dietary advice in avoiding certain products that are generally considered healthy.

Moreover, genetic profiles will help to identify NAFLD patients prone to rapid NASH progression. Various genetic variants influencing the susceptibility to NAFLD and NASH development in both childhood and adulthood have now been identified. With the feasibility of large genetic screens, new insights can be expected from whole exome 
sequencing, for example in confirmed cases of NASH and their matched, healthy controls of same ethnicity, and also in animal models of $\operatorname{NAFLD}(97,98)$.

Taken together, factors associated with NAFLD development and progression become increasingly clear from the large number of studies focusing on NAFLD as an important health threat worldwide for now and future generations. Based on these studies, we conclude that the human body has different ways to handle excess nutrients in order to minimize damage. Whereas excessive glucose and sucrose lead to carbohydrate burden which lead to diabetes mellitus, carbohydrates like fructose result in lipid burden. FAs are stored in the liver and adipose tissue as TGs, lowering toxic FA intermediates. However, TG storage leads to adipose tissue inflammation, which increases the risk of developing the metabolic syndrome. Accordingly, elevated FA $\beta$-oxidation reduces FA concentrations, but increases oxidative damage, accelerating progression to NASH. Alternatively, FAs can be exported from the liver as VLDL, decreasing the risk of progression to NASH, but worsening the cardiovascular risk profile. Strain on specific pathways will determine the symptoms in individual patients. Because each strategy to handle excess nutrients comes at a price, optimizing the balance between energy intake and expenditure still seems the most effective therapeutic option. 


\section{References}

1. Loomba R, Sirlin CB, Schwimmer JB, Lavine JE. Advances in pediatric nonalcoholic fatty liver disease. Hepatology. 2009;50(4):1282-1293. doi:10.1002/hep.23119

2. Tiniakos DG, Vos MB, Brunt EM. Nonalcoholic Fatty Liver Disease: Pathology and Pathogenesis. Annu Rev Pathol Mech Dis. 2010;5(1):145-171. doi:10.1146/annurev-pathol121808-102132

3. Cohen JC, Horton JD, Hobbs HH. Human Fatty Liver Disease: Old Questions and New Insights. Science (80- ). 2011;332(6037):1519-1523. doi:10.1126/science.1204265

4. Brunt E. Histological assessment of nonalcoholic fatty liver disease in adults and children. Clin Liver Dis. 2012;1(4):108-111. doi:10.1002/cld.31

5. Younossi ZM, Koenig AB, Abdelatif D, Fazel Y, Henry L, Wymer M. Global epidemiology of nonalcoholic fatty liver disease-Meta-analytic assessment of prevalence, incidence, and outcomes. Hepatology. 2016;64(1):73-84. doi:10.1002/hep.28431

6. Anderson EL, Howe LD, Jones HE, Higgins JPT, Lawlor DA, Fraser A. The Prevalence of NonAlcoholic Fatty Liver Disease in Children and Adolescents: A Systematic Review and MetaAnalysis. PLoS One. 2015;10. doi:10.1371/journal.pone.0140908

7. Schwimmer JB, Deutsch R, Kahen T, Lavine JE, Stanley C, Behling C. Prevalence of fatty liver in children and adolescents. Pediatrics. 2006;118(4):1388-1393. doi:10.1542/peds.20061212

8. Giorgio V, Prono F, Graziano F, Nobili V. Pediatric non alcoholic fatty liver disease: old and new concepts on development, progression, metabolic insight and potential treatment targets. BMC Pediatr. 2013;13(1):40. doi:10.1186/1471-2431-13-40

9. Speliotes EK, Yerges-Armstrong LM, Wu J, et al. Genome-wide association analysis identifies variants associated with nonalcoholic fatty liver disease that have distinct effects on metabolic traits. PLoS Genet. 2011;7(3). doi:10.1371/journal.pgen.1001324

10. Tilg $\mathrm{H}$, Moschen AR. Evolution of inflammation in nonalcoholic fatty liver disease: The multiple parallel hits hypothesis. Hepatology. 2010;52(5):1836-1846. doi:10.1002/hep.24001

11. Dowman JK, Tomlinson JW, Newsome PN. Pathogenesis of non-alcoholic fatty liver disease. Q J Med. 2010;103(2):71-83. doi:10.1093/qjmed/hcp158

12. Li Y, Ding L, Hassan W, Abdelkader D, Shang J. Adipokines and hepatic insulin resistance. J Diabetes Res. 2013;2013:170532. doi:10.1155/2013/170532

13. Yilmaz Y. Review article: is non-alcoholic fatty liver disease a spectrum, or are steatosis and non-alcoholic steatohepatitis distinct conditions? Aliment Pharmacol Ther. 2012;36(9):815823. doi:10.1111/apt.12046

14. Dhaliwal J, Chavhan GB, Lurz E, et al. Hepatic steatosis is highly prevalent across the paediatric age spectrum, including in pre-school age children. Aliment Pharmacol Ther. 2018;48(5):556-563. doi:10.1111/apt.14900

15. Pandey S. Association of Nonalcoholic Fatty Liver Disease With Relative Skeletal Muscle Mass: APublic Health Perspective. Hepatology. 2018;68(4):1657-1657. doi:10.1002/hep.30107

16. Perry RJ, Samuel VT, Petersen KF, Shulman GI. The role of hepatic lipids in hepatic insulin resistance and type 2 diabetes. Nature. 2014;510(7503):84-91. doi:10.1038/nature13478

17. Cantley JL, Yoshimura T, Camporez JPG, et al. CGI-58 knockdown sequesters diacylglycerols in lipid droplets/ER-preventing diacylglycerol-mediated hepatic insulin resistance. Proc Natl Acad Sci. 2013;110(5):1869-1874. doi:10.1073/pnas.1219456110

18. Duarte N, Coelho IC, Patarrão RS, Almeida JI, Penha-Gonçalves C, Macedo MP. How Inflammation Impinges on NAFLD: A Role for Kupffer Cells. Biomed Res Int. 2015;2015:1-11. 
doi:10.1155/2015/984578

19. Moschen AR, Molnar C, Geiger S, et al. Anti-inflammatory effects of excessive weight loss: potent suppression of adipose interleukin 6 and tumour necrosis factor expression. Gut. 2010;59(9):1259-1264. doi:10.1136/gut.2010.214577

20. Li Z, Yang S, Lin H, et al. Probiotics and antibodies to TNF inhibit inflammatory activity and improve nonalcoholic fatty liver disease. Hepatology. 2003;37(2):343-350. doi:10.1053/jhep.2003.50048

21. Nicholas DA, Zhang $\mathrm{K}$, Hung $\mathrm{C}$, et al. Palmitic acid is a toll-like receptor 4 ligand that induces human dendritic cell secretion of IL-1 $\beta$. PLoS One. 2017;12(5):1-24. doi:10.1371/journal.pone.0176793

22. Pandey S, Agrawal DK. Immunobiology of Toll-like receptors: Emerging trends. Immunol Cell Biol. 2006;84(4):333-341. doi:10.1111/j.1440-1711.2006.01444.x

23. Sharma M, Mitnala S, Vishnubhotla RK, Mukherjee R, Reddy DN, Rao PN. The Riddle of Nonalcoholic Fatty Liver Disease: Progression From Nonalcoholic Fatty Liver to Nonalcoholic Steatohepatitis. J Clin Exp Hepatol. 2015;5(2):147-158. doi:10.1016/j.jceh.2015.02.002

24. Takaki A, Kawai D, Yamamoto K. Multiple hits, including oxidative stress, as pathogenesis and treatment target in non-alcoholic steatohepatitis (NASH). Int J Mol Sci. 2013;14(10):20704-20728. doi:10.3390/ijms141020704

25. Tsukumo DMLD, Carvalho-Filho MA, Carvalheira JJBC, et al. Loss-of-function mutation in Toll-like receptor 4 prevents diet-induced obesity and insulin resistance. Diabetes. 2007;56(August):1986-1998. doi:10.2337/db06-1595.CLS

26. Csak T, Velayudham A, Hritz I, et al. Deficiency in myeloid differentiation factor-2 and tolllike receptor 4 expression attenuates nonalcoholic steatohepatitis and fibrosis in mice. Am J Physiol Liver Physiol. 2011. doi:10.1152/ajpgi.00163.2009

27. Polyzos SA, Kountouras J, Mantzoros CS. Leptin in nonalcoholic fatty liver disease: A narrative review. Metabolism. 2015;64(1):60-78. doi:10.1016/j.metabol.2014.10.012

28. Barshop NJ, Sirlin CB, Schwimmer JB, Lavine JE. Review article: Epidemiology, pathogenesis and potential treatments of paediatric non-alcoholic fatty liver disease. Aliment Pharmacol Ther. 2008;28(1):13-24. doi:10.1111/j.1365-2036.2008.03703.x

29. Awazawa $M$, Ueki $K$, Inabe $K$, et al. Adiponectin enhances insulin sensitivity by increasing hepatic IRS-2 expression via a macrophage-derived IL-6-dependent pathway. Cell Metab. 2011;13(4):401-412. doi:10.1016/j.cmet.2011.02.010

30. Moschen AR, Wieser V, Tilg H. Adiponectin: key player in the adipose tissue-liver crosstalk. Curr Med Chem. 2012;19(32):5467-5473. doi:CMC-EPUB-20120809-7 [pii]

31. Mandal P, Park PH, McMullen MR, Pratt BT, Nagy LE. The anti-inflammatory effects of adiponectin are mediated via a heme oxygenase-1-dependent pathway in rat kupffer cells. Hepatology. 2010;51(4):1420-1429. doi:10.1002/hep.23427

32. Yamaguchi K, Yang L, McCall S, et al. Inhibiting triglyceride synthesis improves hepatic steatosis but exacerbates liver damage and fibrosis in obese mice with nonalcoholic steatohepatitis. Hepatology. 2007;45(6):1366-1374. doi:10.1002/hep.21655

33. Li ZZ, Berk M, McIntyre TM, Feldstein AE. Hepatic lipid partitioning and liver damage in nonalcoholic fatty liver disease: Role of stearoyl-Coa desaturase. J Biol Chem. 2009;284(9):5637-5644. doi:10.1074/jbc.M807616200

34. Akazawa Y, Nakao K. To die or not to die: death signaling in nonalcoholic fatty liver disease. J Gastroenterol. 2018;53(8):893-906. doi:10.1007/s00535-018-1451-5

35. Win S, Than TA, Le BHA, García-Ruiz C, Fernandez-Checa JC, Kaplowitz N. Sab (Sh3bp5) dependence of JNK mediated inhibition of mitochondrial respiration in palmitic acid induced hepatocyte lipotoxicity. J Hepatol. 2015;62(6):1367-1374. doi:10.1016/j.jhep.2015.01.032

36. Paik Y-H, Kim J, Aoyama T, De Minicis S, Bataller R, Brenner DA. Role of NADPH Oxidases in 
Liver Fibrosis. Antioxid Redox Signal. 2014;20(17):2854-2872. doi:10.1089/ars.2013.5619

37. Loffredo L, Zicari AM, Perri L, et al. Does Nox2 Overactivate in Children with Nonalcoholic Fatty Liver Disease? Antioxid Redox Signal. 2018;00(00):ars.2018.7596. doi:10.1089/ars.2018.7596

38. Putignani L, Mosca A, Chierico F Del, et al. Bifidobacteria and lactobacilli in the gut microbiome of children with non-alcoholic fatty liver disease: which strains act as health players? Arch Med Sci. 2018;14(1):81-87. doi:10.5114/aoms.2016.62150

39. Miele L, Valenza V, La Torre G, et al. Increased intestinal permeability and tight junction alterations in nonalcoholic fatty liver disease. Hepatology. 2009;49(6):1877-1887. doi:10.1002/hep.22848

40. Poeta M, Pierri L, Vajro P. Gut-Liver Axis Derangement in Non-Alcoholic Fatty Liver Disease. Children. 2017;4(8):66. doi:10.3390/children4080066

41. Michail $\mathrm{S}$, Lin $\mathrm{M}$, Frey MR, et al. Altered gut microbial energy and metabolism in children with non-alcoholic fatty liver disease. FEMS Microbiol Ecol. 2015;91(2):1-9. doi:10.1093/femsec/fiu002

42. Amar J, Burcelin R, Ruidavets JB, et al. Energy intake is associated with endotoxemia in apparently healthy men. Am J Clin Nutr. 2008;87(5):1219-1223. doi:87/5/1219 [pii]

43. Spruss A, Kanuri G, Wagnerberger S, Haub S, Bischoff SC, Bergheim I. Toll-like receptor 4 is involved in the development of fructose-induced hepatic steatosis in mice. Hepatology. 2009;50(4):1094-1104. doi:10.1002/hep.23122

44. Engstler AJ, Aumiller T, Degen C, et al. Insulin resistance alters hepatic ethanol metabolism: studies in mice and children with non-alcoholic fatty liver disease. Gut. 2016;65(9):15641571. doi:10.1136/gutjnl-2014-308379

45. Famouri F, Shariat Z, Hashemipour M, Keikha M, Kelishadi R. Effects of probiotics on nonalcoholic fatty liver disease in obese children and adolescents. J Pediatr Gastroenterol Nutr. 2017;64(3):413-417. doi:10.1097/MPG.0000000000001422

46. Alisi A, Bedogni G, Baviera G, et al. Randomised clinical trial: The beneficial effects of VSL\#3 in obese children with non-alcoholic steatohepatitis. Aliment Pharmacol Ther. 2014;39(11):1276-1285. doi:10.1111/apt.12758

47. Hatton G, Alterio T, Nobili V, Mann JP. Unmet needs in pediatric NAFLD research: what do we need to prioritize for the future? Expert Rev Gastroenterol Hepatol. 2018;0(0):1-7. doi:10.1080/17474124.2018.1512853

48. Molleston JP, Schwimmer JB, Yates KP, et al. Histological abnormalities in children with nonalcoholic fatty liver disease and normal or mildly elevated alanine aminotransferase levels. J Pediatr. 2014;164(4):707-713.e3. doi:10.1016/j.jpeds.2013.10.071

49. Takahashi Y, Inui A, Fujisawa T, Takikawa H, Fukusato T. Histopathological characteristics of non-alcoholic fatty liver disease in children: Comparison with adult cases. Hepatol Res. 2011;41:1066-1074. doi:10.1111/j.1872-034X.2011.00855.x

50. Takahashi Y, Fukusato T. Pediatric nonalcoholic fatty liver disease: Overview with emphasis on histology. World J Gastroenterol. 2010;16(42):5280. doi:10.3748/wjg.v16.i42.5280

51. Mann JP, De Vito R, Mosca A, et al. Portal inflammation is independently associated with fibrosis and metabolic syndrome in pediatric nonalcoholic fatty liver disease. Hepatology. 2016;63(3):745-753. doi:10.1002/hep.28374

52. Thoolen B, Maronpot RR, Harada T, et al. Proliferative and nonproliferative lesions of the rat and mouse hepatobiliary system. Toxicol Pathol. 2010;38(7 Suppl):5S-81S. doi:10.1177/0192623310386499

53. Nobili V, Mosca A, De Vito R, Raponi M, Scorletti E, Byrne CD. Liver zonation in children with non-alcoholic fatty liver disease: Associations with dietary fructose and uric acid concentrations. Liver Int. 2018;38(6):1102-1109. doi:10.1111/liv.13661

54. Lake AA, Mathers JC, Rugg-Gunn AJ, Adamson AJ. Longitudinal change in food habits 
between adolescence (11-12 years) and adulthood (32-33 years): The ASH30 study. J Public Health (Bangkok). 2006;28(1):10-16. doi:10.1093/pubmed/fdi082

55. Goran MI, Gover BA, Nagy TR, Johnson RK. Developmental changes in energy expenditure and physical activity in children: Evidence for a decline in physical activity in girls before puberty. Pediatrics. 1998;101(5):887-891. doi:10.1542/peds.101.5.887

56. Leech RM, McNaughton $S$ a, Timperio A. The clustering of diet, physical activity and sedentary behavior in children and adolescents: a review. Int J Behav Nutr Phys Act. 2014;11:4. doi:10.1186/1479-5868-11-4

57. Valtueña S, Pellegrini N, Ardigò D, et al. Dietary glycemic index and liver steatosis. Am J Clin Nutr. 2006;84(1):136-142. doi:10.1093/ajcn/84.1.136

58. Mosca A, Nobili V, De Vito $R$, et al. Serum uric acid concentrations and fructose consumption are independently associated with NASH in children and adolescents. J Hepatol. 2017;66(5):1031-1036. doi:10.1016/j.jhep.2016.12.025

59. Tappy L, Le K-A. Metabolic Effects of Fructose and the Worldwide Increase in Obesity. Physiol Rev. 2010;90(1):23-46. doi:10.1152/physrev.00019.2009

60. Sluik D, Engelen Al, Feskens EJ. Fructose consumption in the Netherlands: the Dutch national food consumption survey 2007 - 2010. Eur J Clin Nutr. 2015;69(4):475-481. doi:10.1038/ejcn.2014.267

61. Mosca A, Della C, Maria C, et al. Beverage consumption and paediatric NAFLD. Eat Weight Disord - Stud Anorexia, Bulim Obes. 2016;21(4):581-588. doi:10.1007/s40519-016-0315-3

62. Ouyang $X$, Cirillo $P$, Sautin $Y$, et al. Fructose consumption as a risk factor for non-alcoholic fatty liver disease. J Hepatol. 2008;48(6):993-999. doi:10.1016/j.jhep.2008.02.011

63. Schwarz J, Noworolski SM, Erkin-Cakmak A, et al. Effects of Dietary Fructose Restriction on Liver Fat, De Novo Lipogenesis, and Insulin Kinetics in Children With Obesity. Gastroenterology. 2017;153(3):743-752. doi:10.1053/j.gastro.2017.05.043

64. Stanhope KL, Havel PJ. Fructose consumption: Recent results and their potential implications. Ann N Y Acad Sci. 2010;1190:15-24. doi:10.1111/j.1749-6632.2009.05266.x

65. Stanhope KL, Schwarz JM, Keim NL, et al. Consuming fructose-sweetened, not glucosesweetened, beverages increases visceral adiposity and lipids and decreases insulin sensitivity in overweight/obese humans. J Clin Invest. 2009;119(5):1322-1334. doi:10.1172/JCl37385

66. Kelly LA, Lane CJ, Weigensberg MJ, Toledo-Corral CM, Goran MI. Pubertal Changes of Insulin Sensitivity, Acute Insulin Response, and $\beta$-Cell Function in Overweight Latino Youth. J Pediatr. 2011;158(3):442-446. doi:10.1016/j.jpeds.2010.08.046

67. Mager DR, Patterson C, So S, Rogenstein CD, Wykes $L$, Roberts E a. Dietary and physical activity patterns in children with fatty liver. Eur J Clin Nutr. 2010;64(6):628-635. doi:10.1038/ejcn.2010.35

68. Xu L, Li M, Yin J, et al. Change of Body Composition and Adipokines and Their Relationship with Insulin Resistance across Pubertal Development in Obese and Nonobese Chinese Children: The BCAMS Study. Int J Endocrinol. 2012;2012:1-10. doi:10.1155/2012/389108

69. Sperling MA. Traditional and novel aspects of the metabolic actions of growth hormone. Growth Horm IGF Res. 2016;28:69-75. doi:10.1016/j.ghir.2015.06.005

70. Vijayakumar A, Yakar S, LeRoith D. The Intricate Role of Growth Hormone in Metabolism. Front Endocrinol (Lausanne). 2011;2(sep):1-11. doi:10.3389/fendo.2011.00032

71. Takahashi Y. The role of growth hormone and insulin-like growth factor-I in the liver. Int J Mol Sci. 2017;18(7). doi:10.3390/ijms18071447

72. $\mathrm{Xu} \mathrm{L,} \mathrm{Xu} \mathrm{C,} \mathrm{Yu} \mathrm{C,} \mathrm{et} \mathrm{al.} \mathrm{Association} \mathrm{between} \mathrm{Serum} \mathrm{Growth} \mathrm{Hormone} \mathrm{Levels} \mathrm{and}$ Nonalcoholic Fatty Liver Disease: A Cross-Sectional Study. PLoS One. 2012;7(8). doi:10.1371/journal.pone.0044136

73. Dichtel LE, Corey KE, Misdraji J, et al. The association between igf-1 levels and the histologic 
severity of nonalcoholic fatty liver disease. Clin Transl Gastroenterol. 2017;8(1):1-9. doi:10.1038/ctg.2016.72

74. Takahashi Y. Essential roles of growth hormone (GH) and insulin-like growth factor-I (IGF-I) in the liver. Endocr J. 2012;59(11):955-962. doi:10.1507/endocrj.EJ12-0322

75. Lewitt M, Dent M, Hall K. The Insulin-Like Growth Factor System in Obesity, Insulin Resistance and Type 2 Diabetes Mellitus. J Clin Med. 2014;3(4):1561-1574. doi:10.3390/jcm3041561

76. Denzer C, Thiere D, Muche R, et al. Gender-specific prevalences of fatty liver in obese children and adolescents: roles of body fat distribution, sex steroids, and insulin resistance. J Clin Endocrinol Metab. 2009;94(10):3872-3881. doi:10.1210/jc.2009-1125

77. Meyer MR, Clegg DJ, Prossnitz ER, Barton M. Obesity, insulin resistance and diabetes: sex differences and role of oestrogen receptors. Acta Physiol. 2011;203(1):259-269. doi:10.1111/j.1748-1716.2010.02237.x

78. Brown L, Clegg D. Central Effects of Estradiol in the Regulation of Adiposity. J Steroid Biochem Mol Biol. 2010;122((1-3)):65-73. doi:10.1016/j.jsbmb.2009.12.005.Central

79. Söderberg $\mathrm{S}$, Olsson $\mathrm{T}$, Eliasson $\mathrm{M}$, et al. A strong association between biologically active testosterone and leptin in non-obese men and women is lost with increasing (central) adiposity. Int J Obes Relat Metab Disord. 2001;25(1):98-105. doi:10.1038/sj.ijo.0801467

80. Böttner A, Kratzsch J, Müller G, et al. Gender differences of adiponectin levels develop during the progression of puberty and are related to serum androgen levels. J Clin Endocrinol Metab. 2004;89(8):4053-4061. doi:10.1210/jc.2004-0303

81. Hui ST, Kurt Z, Tuominen I, et al. The Genetic Architecture of Diet-Induced Hepatic Fibrosis in Mice. Hepatology. 2018;68(6):2182-2196. doi:10.1002/hep.30113

82. Sookoian S, Pirola CJ. Meta-analysis of the influence of I148M variant of patatin-like phospholipase domain containing 3 gene (PNPLA3) on the susceptibility and histological severity of nonalcoholic fatty liver disease. Hepatology. 2011;53(6):1883-1894. doi:10.1002/hep.24283

83. Trépo E, Romeo S, Zucman-Rossi J, Nahon P. PNPLA3 gene in liver diseases. J Hepatol. 2016;65(2):399-412. doi:10.1016/j.jhep.2016.03.011

84. Krawczyk M, Portincasa P, Lammert F. PNPLA3 -associated steatohepatitis: Toward a genebased classification of fatty liver disease. Semin Liver Dis. 2013;33(4):369-379. doi:10.1055/s-0033-1358525

85. Smagris E, Basuray S, Li J, et al. Pnpla3I148M knockin mice accumulate PNPLA3 on lipid droplets and develop hepatic steatosis. Hepatology. 2015;61(1):108-118. doi:10.1002/hep.27242

86. He S, McPhaul C, Li JZ, et al. A sequence variation (I148M) in PNPLA3 associated with nonalcoholic fatty liver disease disrupts triglyceride hydrolysis. J Biol Chem. 2010;285(9):6706-6715. doi:10.1074/jbc.M109.064501

87. Goran MI, Walker R, Le KA, et al. Effects of PNPLA3 on liver fat and metabolic profile in hispanic children and adolescents. Diabetes. 2010;59(12):3127-3130. doi:10.2337/db100554

88. Liu YL, Patman GL, Leathart JBS, et al. Carriage of the PNPLA3 rs738409 C >g polymorphism confers an increased risk of non-alcoholic fatty liver disease associated hepatocellular carcinoma. J Hepatol. 2014. doi:10.1016/j.jhep.2014.02.030

89. Beer NL, Tribble ND, McCulloch $\mathrm{L}$, et al. The P446L variant in GCKR associated with fasting plasma glucose and triglyceride levels exerts its effect through increased glucokinase activity in liver. Hum Mol Genet. 2009. doi:10.1093/hmg/ddp357

90. Santoro $\mathrm{N}$, Zhang $\mathrm{CK}$, Zhao H, et al. Variant in the glucokinase regulatory protein (GCKR) gene is associated with fatty liver in obese children and adolescents. Hepatology. 2012. doi:10.1002/hep.24806 
91. Chen L-Z, Hua-Xiang Xia H, Xin Y-N, Lin Z-H, Xuan S-Y. TM6SF2 E167K Variant, a Novel Genetic Susceptibility Variant, Contributing to Nonalcoholic Fatty Liver Disease. J Clin TransI Hepatol. 2015;3(4):265-270. doi:10.14218/JCTH.2015.00023

92. Goffredo M, Caprio S, Feldstein AE, et al. Role of TM6SF2 rs58542926 in the pathogenesis of nonalcoholic pediatric fatty liver disease: A multiethnic study. Hepatology. 2016;63(1):117125. doi:10.1002/hep.28283

93. Pirola CJ, Sookoian S. The dual and opposite role of the TM6SF2-rs58542926 variant in protecting against cardiovascular disease and conferring risk for nonalcoholic fatty liver: A meta-analysis. Hepatology. 2015;62(6):1742-1756. doi:10.1002/hep.28142

94. Grandone A, Cozzolino D, Marzuillo P, et al. TM6SF2 Glu167Lys polymorphism is associated with low levels of LDL-cholesterol and increased liver injury in obese children. Pediatr Obes. 2016;11(2):115-119. doi:10.1111/ijpo.12032

95. Viitasalo A, Eloranta AM, Atalay M, Romeo S, Pihlajamäki J, Lakka TA. Association of MBOAT7 gene variant with plasma ALT levels in children: The PANIC study. Pediatr Res. 2016. doi:10.1038/pr.2016.139

96. Di Costanzo A, Belardinilli F, Bailetti D, et al. Evaluation of Polygenic Determinants of NonAlcoholic Fatty Liver Disease (NAFLD) By a Candidate Genes Resequencing Strategy. Sci Rep. 2018. doi:10.1038/s41598-018-21939-0

97. Pandey S. The Genetic Architecture of Diet-Induced Hepatic Fibrosis in Mice: A Public Health Perspective. Hepatology. 2018;68(6):2441-2442. doi:10.1002/hep.30207

98. Pandey S. Healthy controls in nonalcoholic fatty liver disease management: A biomedical research perspective. Hepatology. 2017;66(3):1006-1007. doi:10.1002/hep.29334

99. Horlick $M B$, Rosenbaum $M$, Nicholson $M$, et al. Effect of Puberty on the Relationship between Circulating Leptin and Body Composition. J Clin Endocrinol Metab. 2000;85(7):2509-2518. doi:10.1210/jcem.85.7.6689

100. Ellis KJ, Nicolson M. Leptin Levels and Body Fatness in Children: Effects of Gender, Ethnicity, and Sexual Development1. Pediatr Res. 1997;42(4):484-488. doi:10.1203/00006450199710000-00010

101. Martos-Moreno GÁ, Barrios V, Argente J. Normative data for adiponectin, resistin, interleukin 6 and leptin/receptor ratio in a healthy Spanish pediatric population: Relationship with sex steroids. Eur J Endocrinol. 2006;155(3):429-434. doi:10.1530/eje.1.02227

102. Andersen KK, Frystyk J, Wolthers OD, Heuck C, Flyvbjerg A. Gender differences of oligomers and total adiponectin during puberty: A cross-sectional study of 859 Danish School Children. J Clin Endocrinol Metab. 2007;92(5):1857-1862. doi:10.1210/jc.2006-2310

103. Sehested A, Juul A, Andersson AM, et al. Serum inhibin A and inhibin B in healthy prepubertal, pubertal, and adolescent girls and adult women: Relation to age, stage of puberty, menstrual cycle, follicle-stimulating hormone, luteinizing hormone, and estradiol levels. J Clin Endocrinol Metab. 2000;85(4):1634-1640. doi:10.1210/jc.85.4.1634

104. Konforte D, Shea JL, Kyriakopoulou L, et al. Complex biological pattern of fertility hormones in children and adolescents: A study of healthy children from the CALIPER cohort and establishment of pediatric reference intervals. Clin Chem. 2013;59(8):1215-1227. doi:10.1373/clinchem.2013.204123 


\section{Chapter 6}

Factors associated with increasing degrees of hepatic steatosis and progression to steatohepatitis in children with overweight or obesity

Kylie Karnebeek Jogchum Plat

Simon Robben Anita C.E. Vreugdenhil 


\section{Abstract}

\section{Background and aims}

Nonalcoholic fatty liver disease (NAFLD) encompasses a spectrum of liver disorders, which is assumed to start with hepatic steatosis, and progresses to steatohepatitis, liver fibrosis and even liver cirrhosis in a selection of patients. Factors associated with this progression to the next stages in the NAFLD spectrum in children and adults have not been well elucidated. The aim of this study was to identify determinants that associate with increasing degrees of hepatic steatosis, as estimated with the ultrasonographic hepatorenal index (HRI), in children with overweight, obesity and morbid obesity, and to identify which factors contribute to further progression from steatosis to steatohepatitis, as measured with alanine aminotransferase (ALT) concentrations.

\section{Methods}

Two hundred and fifty-eight children (49\% male) with overweight, obesity and morbid obesity from the Centre for Overweight Adolescent and Children's Healthcare were included. The hepatorenal index was calculated as the ratio between ultrasonographic hepatic and renal echogenicity. Anthropometric, metabolic, cardiovascular and liverrelated parameters were measured.

\section{Results}

Multiple linear regression analysis $\left(R^{2}=0.263\right)$ identified ALT concentrations $(\beta=0.003$, $p<0.001)$, age $(\beta=-0.007, p=0.001)$, fasting glucose concentrations $(\beta=0.025, p=0.039)$ and CRP concentrations $(\beta=-0.002, p=0.042)$ as factors associated with logHRI. There was a trend towards a higher percentage of boys with a high HRI, compared to boys with a low HRI.

In the whole group, HRI $(\beta=0.165, p<0.001)$, male sex $(\beta=0.068, p=0.015)$, BMI $z$-score $(\beta=0.059, p=0.004)$, serum triglyceride concentrations $(\beta=0.073, p=0.006)$ and ferritin concentrations $(\beta=0.001, p=0.036)$ were identified as factors associated with $\log A L T$ concentrations. In the children with severe hepatic steatosis, HRI concentrations $(\beta=0.188$, $p<0.001)$, fasting insulin concentrations $(\beta=-0.002, p=0.026)$, triglyceride concentrations $(\beta=0.087, p=0.037)$ and ferritin concentrations $(\beta=0.003, p<0.001)$ were significantly associated with logALT concentrations.

\section{Conclusion}

Age, glucose metabolism and inflammatory status are factors associated with an increasing degree of hepatic steatosis, whereas the amount of fat accumulation in the 
liver, sex, severity of overweight, triglyceride concentrations and ferritin concentrations are important factors associated with progression to steatohepatitis in children with overweight and obesity. This data provides important insight into the factors that are associated with the consecutive NAFLD stages in children with overweight and obesity and underline that it is important to study determinants associated with the different stage of disease severity separately. 


\section{Introduction}

Nonalcoholic fatty liver disease (NAFLD) is a common liver disease in children. The term NAFLD is used to describe a range of liver disorders with increasing severity. Hepatic steatosis is often regarded as the first stage of the spectrum, but the disease progresses to steatohepatitis (NASH), liver fibrosis and even liver cirrhosis in a subgroup of patients. Progression to the severe end of the disease spectrum occurs more rapidly in some patients compared to others (1). This progressive nature of NAFLD and the fact that it has been associated with an increased risk of developing hepatocellular carcinoma (2), diabetes type II (3) and cardiovascular disease (4), makes it an important health threat.

The underlying pathophysiological mechanisms of NAFLD development and progression are still incompletely understood, but it is clear that NAFLD is the consequence of a complex interplay of multifactorial processes. Obesity is assumed to be a driving factor behind the increasing prevalence of NAFLD, as NAFLD is more common in obese children (34\%) than in children from the general population (8\%)(5). Additionally, metabolic aberrations like insulin resistance and dyslipidemia $(6,7)$, genetic predisposition $(8,9)$, gut barrier dysfunction (10), dietary habits (11, 12), low-grade inflammation (13), sex (14) and ethnicity (15) have been associated with NAFLD development and progression. However, it is not yet fully understood which of these factors are associated with the different stages of disease severity and the pace at which someone advances through the stages.

In order to better understand the course of disease and its underlying mechanisms, valid non-invasive methods to determine and quantify the severity of the different disease stages are necessary. Currently, the gold standard for diagnosing and staging nonalcoholic fatty liver disease is liver biopsy, which is an invasive procedure with a small risk of severe complications making it unsuitable for screening purposes or research in children. ${ }^{1} \mathrm{H}$ magnetic resonance spectroscopy $\left({ }^{1} \mathrm{H}-\mathrm{MRS}\right)$ is a technique that was shown to have a high sensitivity to quantitatively measure hepatic fat content (16) and is used in clinical and research settings, however measurement can be demanding for children. The ultrasonographic hepatorenal index ( $\mathrm{HRI})$ is an easy and non-invasive measurement to quantitatively assess hepatic steatosis $(17,18)$, that was shown to have a high agreement with liver fat content estimated with liver biopsy and ${ }^{1} \mathrm{H}-\mathrm{MRS}(17,19-21)$. The fact that ultrasonography is less of a burden for children to undergo, is an advantage of the HRI compared to ${ }^{1} \mathrm{H}$-MRS when studying large groups of children.

Since ${ }^{1} \mathrm{H}-\mathrm{MRS}$ and HRI measure liver fat content, but not hepatic inflammation, these methods are not appropriate for assessing steatohepatitis. Therefore, surrogate markers 
for steatohepatitis such as alanine aminotransferase (ALT) concentrations are commonly used in clinical and research settings. ALT is released as a consequence of hepatocellular injury (22) and elevated concentrations are therefore not limited to NASH. Excluding other causes of chronic liver disease is recommended in children with persistently elevated ALT concentrations (23). Therefore, in the absence of other chronic liver disease and in the presence of overweight or obesity, ALT concentrations can be used as a marker for NASH. A recent meta-analysis of adult studies reported a pooled sensitivity of $65 \%$ and pooled specificity of $75 \%$ for discriminating NASH from hepatic steatosis (24).

As progression from steatosis to more severe NAFLD disease stages starts with steatohepatitis, gaining more insight in the factors associated with steatohepatitis might also help in a better understanding of the factors that are associated with progression along the entire disease spectrum. Therefore, in this study we aimed to identify factors associated with increasing degrees of hepatic steatosis, as measured with the ultrasonographic hepatorenal index, in children with overweight, obesity and morbid obesity, and to identify which factors are associated with progression to steatohepatitis, as assessed by alanine aminotransferase concentrations. Elaborate anthropometric and cardiometabolic parameters were analyzed and taken into account.

\section{Methods}

\section{Participants}

This study was performed within the Centre for Overweight Adolescent and Children's Healthcare $(\mathrm{COACH})$ at the Maastricht University Medical Centre. In this obesity expertise centre, children and adolescents with overweight, obesity and morbid obesity are evaluated, monitored and guided as described previously in detail (25). The study was conducted according to the guidelines of the Declaration of Helsinki and approved by the medical ethical committee of the MUMC+.

\section{Liver ultrasonography}

Ultrasonography was performed using the iU22 ultrasound system (Philips Healthcare, Eindhoven, The Netherlands) using a broad-band C5-1 curved-array transducer. All images were reviewed by a pediatric radiologist with 30 years of experience in pediatric ultrasonography. 
To determine the hepatorenal index, the probe was placed in a right subcostal or intercostal position to obtain three sagittal images of both the liver and renal parenchyma. In each image, a region of interest of the liver and renal parenchyma, excluding bile ducts and blood vessels, was determined. In these regions of interest, the mean gray-scale intensity of the liver and renal parenchyma was determined and the average of three measurements was used to calculate the hepatorenal index by the following formula:

$$
H R I=\frac{\text { echogenicity of the liver }}{\text { echogenicity of the kidney }}
$$

Currently, there are no references for normal or elevated HRI values in children. Therefore, the degree of liver fat content in children was estimated by dividing the children into three groups: children with none to mild hepatic steatosis $(0.86-1.30$, i.e. the lowest HRI tertile), children with moderate hepatic steatosis (1.31-1.60, i.e. the middle HRI tertile) and children with severe hepatic steatosis (1.61-4.40, i.e. the highest HRI tertile).

\section{Anthropometric measurements}

Anthropometric measurements were performed while children were barefoot, wearing only underwear. Weight was measured on a digital scale (Seca) and height was measured using a digital stadiometer (De Grood Metaaltechniek). Subsequently, Body Mass Index (BMI) was calculated and BMI z-scores were obtained using a growth analyser (Growth Analyser VE).

\section{Parameters reflecting liver health}

Serum alanine transaminase (ALT), aspartate transaminase (AST), gamma-glutamyl transferase (GGT), alkaline phosphatase (ALP) and serum ferritin concentrations were determined with the Cobas 8000 modular analyser (Roche). Based on a previous study evaluating normal ranges of ALT concentrations in children and the recommendations of the North American Society of Pediatric Gastroenterology, Hepatology and Nutrition (NASPGHAN) guideline for diagnosing NAFLD in children (23), the upper limit of normal (ULN) used for ALT was $22 \mathrm{U} / \mathrm{L}$ for girls and $26 \mathrm{U} / \mathrm{L}$ for boys (26).

\section{Metabolic and cardiovascular parameters}

Fasting serum total cholesterol, HDL-cholesterol, triglycerides, free fatty acids (FFA) and blood glucose concentrations were determined with the Cobas 8000 modular analyser and 
serum insulin concentrations with the Immulite 1000 (Siemens). LDL cholesterol concentrations were calculated using the Friedewald equation (27). After obtaining the fasting blood sample an oral glucose tolerance test (OGTT) was performed. For this test, children consumed 1.75 grams of glucose per kilogram of bodyweight (with a maximum of 75 grams of glucose), dissolved into $200 \mathrm{~mL}$ water. Plasma glucose concentrations measured 120 minutes after consuming the glucose solution were used for further calculations. Moreover, insulin resistance was estimated by calculating the homeostasis model assessment for insulin resistance (HOMA-IR) (HOMA-IR = fasting glucose ( $\mathrm{mmol} / \mathrm{L}) \mathrm{x}$ fasting insulin $(\mu \mathrm{U} / \mathrm{L}) / 22.5)$. Blood pressure was measured approximately 20 times using the Mobil-O-Graph (I.E.M. GmbH), after which the mean blood pressure was calculated. Blood pressure z-scores were calculated according to reference values for height and sex (28).

\section{Statistical analysis}

Statistical analyses were performed with IBM SPSS statistics 25.0. Data was tested for normality with the Shapiro-Wilk test and reported as mean \pm standard deviation or median (interquartile range), as appropriate. Differences in parameters between groups were analyzed with an Independent Samples T-test, Mann-Whitney $U$ test, ANOVA or Kruskal-Wallis test, as appropriate.

Multivariate regression analysis was performed to identify determinants of HRI and ALT concentrations. Possible contributors were identified from correlation analysis and differences between the three liver fat content groups (i.e. significant correlations or groups differences, or trends). For the regression analyses, HRI and ALT concentrations were log-transformed to correct for a skewed distribution.

A p-value $<0.05$ was considered statistically significant and was corrected for multiple testing if applicable.

\section{Results}

Two-hundred and fifty-eight children (49\% boys) with a mean BMI z-score of $3.21 \pm 0.71$ and a median age of $11.9(9.5 ; 14.7)$ years were included in the analysis. The median hepatorenal index was $1.46(1.27 ; 1.70)$ and median ALT concentrations were $23(18 ; 31)$. Fifty-three $\%$ of the children had ALT concentrations within the normal range (i.e. below $26 \mathrm{U} / \mathrm{L}$ for boys and below $22 \mathrm{U} / \mathrm{L}$ for girls), $38 \%$ of the children had ALT concentrations above the upper limit of normal but below twice the upper limit (i.e. 26-52 U/L for boys 
and 22-44 U/L for girls) and $8 \%$ of the children had ALT concentrations above two times the upper limit (table 1).

\section{Differences between children with none-mild, moderate and severe hepatic steatosis}

Children with moderate or severe hepatic steatosis were significantly younger than children with none-mild hepatic steatosis. Children with severe hepatic steatosis had significantly higher ALT concentrations $(24(20 ; 38))$ compared to children with none-mild steatosis $(22(17 ; 28))(p=0.008)$. There was a trend towards higher fasting glucose concentrations in children with a higher degree of hepatic steatosis $(p=0.051)$. Finally, there was a trend towards a different sex distribution between the three hepatic steatosis severity groups, with a higher percentage of girls with none-mild steatosis and a higher percentage of boys with severe hepatic steatosis $(p=0.064)$. The other cardiometabolic parameters that were evaluated did not differ significantly between children with different degrees of hepatic steatosis severities. All characteristics of children with nonemild, moderate or severe hepatic steatosis are shown in table 1.

Table 1. Baseline characteristics for the total group of participants and stratified based on liver fat content

\begin{tabular}{|c|c|c|c|c|}
\hline & $\begin{array}{c}\text { Total } \\
\text { population }\end{array}$ & $\begin{array}{c}\text { None-mild hepatic } \\
\text { steatosis }\end{array}$ & $\begin{array}{c}\text { Moderate hepatic } \\
\text { steatosis }\end{array}$ & $\begin{array}{c}\text { Severe hepatic } \\
\text { steatosis }\end{array}$ \\
\hline HRI & $1.46(1.27 ; 1.70)$ & $1.20(1.08 ; 1.26)$ & $1.46(1.38 ; 1.54)^{\mathrm{A}}$ & $1.89(1.70 ; 2.16)^{B, C}$ \\
\hline$N($ male/female $)$ & $258(127 / 131)$ & $83(32 / 51)$ & $91(48 / 43)$ & $84(47 / 37)$ \\
\hline Age, years & $11.9(9.5 ; 14.7)$ & $13.6(11.4 ; 15.8)$ & $11.2(8.9 ; 13.9)^{\mathrm{A}}$ & $11.5(9.1 ; 14.2)^{\mathrm{B}}$ \\
\hline BMI z-score & $3.21 \pm 0.71$ & $3.18 \pm 0.66$ & $3.20 \pm 0.72$ & $3.27 \pm 0.74$ \\
\hline$A L T, U / L$ & $23(18 ; 31)$ & $22(17 ; 28)$ & $22(17 ; 29)$ & $24(20 ; 38)^{B}$ \\
\hline$A L T<U L N, \%$ & 52.7 & 57.7 & 56.5 & 43.4 \\
\hline ALT 1-2xULN, \% & 38.1 & 38.5 & 36.5 & 39.5 \\
\hline$A L T>2 x \cup L N, \%$ & 9.2 & 3.8 & 7.1 & 17.1 \\
\hline$A S T, U / L$ & $27(22 ; 32)$ & $25.0(21.0 ; 31.0)$ & $26.0(21.2 ; 31.8)$ & $28.0(23.5 ; 34.0)$ \\
\hline$G G T, U / L$ & $16(13 ; 20)$ & $15.0(11.5 ; 21.0)$ & $16.0(14.0 ; 19.0)$ & $15.0(13.0 ; 21.0)$ \\
\hline Alkaline phosphatase, $U / L$ & $233(145 ; 306)$ & $196.5(98.3 ; 262.5)$ & $272.0(207.0 ; 331.3)^{\mathrm{A}}$ & $246.0(160.5 ; 306.0)$ \\
\hline Ferritine, $\mu g / L$ & $47(32 ; 69)$ & $47(31.5 ; 71)$ & $43(32 ; 64)$ & $49(31 ; 74)$ \\
\hline Total cholesterol, $\mathrm{mmol} / \mathrm{L}$ & $4.0(3.6 ; 4.6)$ & $4.0(3.5 ; 4.7)$ & $4.0(3.6 ; 4.5)$ & $4.3(3.6 ; 4.8)$ \\
\hline LDL cholesterol, $\mathrm{mmol} / \mathrm{L}$ & $2.37 \pm 0.68$ & $2.36 \pm 0.69$ & $2.34 \pm 0.62$ & $2.39 \pm 0.76$ \\
\hline HDL cholesterol, $\mathrm{mmol} / \mathrm{L}$ & $1.30(1.10 ; 1.45)$ & $1.20(1.02 ; 1.40)$ & $1.30(1.10 ; 1.50)$ & $1.20(1.10 ; 1.45)$ \\
\hline Triglycerides, $\mathrm{mmol} / \mathrm{L}$ & $0.92(0.66 ; 1.22)$ & $0.94(0.72 ; 1.27)$ & $0.92(0.64 ; 1.16)$ & $0.88(0.64 ; 1.36)$ \\
\hline Free fatty acids, $\mathrm{mmol} / \mathrm{L}$ & $0.74 \pm 0.23$ & $0.75 \pm 0.25$ & $0.74 \pm 0.22$ & $0.71 \pm 0.22$ \\
\hline Fasting glucose, $\mathrm{mmol} / \mathrm{L}$ & $4.5(4.1 ; 4.9)$ & $4.38 \pm 0.53$ & $4.52 \pm 0.56$ & $4.60 \pm 0.67$ \\
\hline 2-hour glucose, $\mathrm{mmol} / \mathrm{L}$ & $5.76 \pm 1.43$ & $5.79 \pm 1.45$ & $5.64 \pm 1.13$ & $5.86 \pm 1.69$ \\
\hline Fasting insulin, $\mu U / L$ & $13.5(8.8 ; 22.0)$ & $13.6(9.4 ; 21.2)$ & $12.8(7.6 ; 20.2)$ & $15.3(8.5 ; 24.9)$ \\
\hline HOMA-IR & $2.71(1.66 ; 4.42)$ & $2.74(1.78 ; 4.13)$ & $2.57(1.46 ; 4.10)$ & $3.11(1.61 ; 5.43)$ \\
\hline Systolic bloodpressure z-score & $0.05(-0.70 ; 0.82)$ & $0.15(-0.62 ; 1.13)$ & $0.04(-0.58 ; 0.67)$ & $0.05(-1.04 ; 0.95)$ \\
\hline Diastolic bloodpressure z-score & $-0.83(-1.62 ;-0.08)$ & $-0.84(-1.41 ; 0.01)$ & $-0.78(-1.63 ;-0.14)$ & $-1.00(-2.00 ;-0.02)$ \\
\hline$C R P, m g / L$ & $2.0(1.0 ; 5.0)$ & $3.0(2.0 ; 7.0)$ & $2.0(1.0 ; 3.0)^{A}$ & $2.0(1.0 ; 4.0)^{\mathrm{B}}$ \\
\hline
\end{tabular}

$A=$ significant difference between children with none-mild hepatic steatosis and children with moderate hepatic steatosis; $B=$ significant difference between children with none-mild hepatic steatosis and children with severe steatosis; $C=$ significant difference between children with moderate hepatic steatosis and children with severe hepatic steatosis. 


\section{Determinants of hepatic steatosis}

In the total group of children, the HRI was positively correlated with ALT and AST concentrations (respectively $R=0.229, p<0.001$ and $R=0.159, p=0.014$ ) and fasting glucose concentrations $(R=0.135, p=0.037)$ and inversely correlated with age $(R=-0.203, p=0.001)$ and CRP ( $R=-0.193, p=0.007)$ (table 2 ). In children with none-mild hepatic steatosis, there were no significant correlations between the HRI and BMI z-score or cardiometabolic parameters. In children with moderate hepatic steatosis, the HRI was positively correlated with $B M I z$-score $(R=0.234, p=0.025)$ and ferritin concentrations $(R=0.212, p=0.043)$. Finally, the HRI was significantly correlated with ALT concentrations $(R=0.543, p<0.001)$, AST concentrations ( $R=0.441, p<0.001)$, GGT concentrations $(R=0.359, p=0.001)$ and ferritin concentrations $(R=0.240, p=0.034)$ in the children with severe hepatic steatosis.

Multiple linear regression analysis identified that $A L T$ concentrations $(\beta=0.003, p<0.001)$, age $(\beta=-0.007, p=0.001)$, fasting glucose concentrations $(\beta=0.025, p=0.039)$ and CRP concentrations $(\beta=-0.002, p=0.042)$ were significantly associated with logHRI $\left(R^{2}=0.263\right)$ (table 3 ). Further elaboration of the inverse association between the logHRI and age showed that this association was driven by an inverse association in girls $(R=-0.327$, $\mathrm{p}<0.001)$, but not boys $(\mathrm{R}=-0.042, \mathrm{p}=0.789)$.

Regression analysis in the children with severe hepatic steatosis, showed that only ALT concentrations $(\beta=0.002, p=0.001)$ were significantly associated with logHRI $\left(R^{2}=0.320\right)$. Finally, in this group with severe hepatic steatosis, there was a trend towards an inverse association between logHRI and CRP concentrations ( $\beta=-0.008, p=0.067$ )(table 3 ). Regression analysis in the children with none-mild hepatic steatosis, performed with variables that were significantly different (or a trend) compared with the other groups did not result in a significant model explaining logHRI. 
Table 2. Correlation coefficients between the hepatorenal index and health parameters, in the total population and stratified based on liver fat content

\begin{tabular}{|c|c|c|c|c|}
\hline & $\begin{array}{c}\text { Total } \\
\text { population }\end{array}$ & $\begin{array}{l}\text { None-mild hepatic } \\
\text { steatosis }\end{array}$ & $\begin{array}{c}\text { Moderate hepatic } \\
\text { steatosis }\end{array}$ & $\begin{array}{c}\text { Severe hepatic } \\
\text { steatosis }\end{array}$ \\
\hline Age, years & $R=-0.203^{A}$ & $R=-0.009$ & $R=-0.166$ & $R=-0.067$ \\
\hline BMI z-score & $R=0.064$ & $R=-0.033$ & $R=0.234^{A}$ & $R=0.057$ \\
\hline$A L T, U / L$ & $R=0.229^{A}$ & $R=0.110$ & $R=0.063$ & $R=0.543^{A}$ \\
\hline$A S T, U / L$ & $R=0.159^{A}$ & $R=-0.034$ & $R=0.085$ & $\mathrm{R}=0,441^{\mathrm{A}}$ \\
\hline$G G T, U / L$ & $R=0.093$ & $R=-0.075$ & $\mathrm{R}=0.011$ & $R=0.359^{A}$ \\
\hline Alkaline phosphatase, $U / L$ & $R=0.044$ & $R=0.022$ & $\mathrm{R}=0.121$ & $R=-0.091$ \\
\hline Ferritin, $\mu g / L$ & $R=0.051$ & $R=-0.090$ & $\mathrm{R}=0.212^{\mathrm{A}}$ & $\mathrm{R}=0.240^{\mathrm{A}}$ \\
\hline Total cholesterol, $\mathrm{mmol} / \mathrm{L}$ & $R=0.039$ & $R=-0.136$ & $R=-0.049$ & $R=0.153$ \\
\hline LDL cholesterol, $\mathrm{mmol} / \mathrm{L}$ & $R=0.027$ & $R=-0.069$ & $R=0.007$ & $R=-0.045$ \\
\hline HDL cholesterol, $\mathrm{mmol} / \mathrm{L}$ & $R=0.038$ & $R=-0.129$ & $R=-0.041$ & $R=0.050$ \\
\hline Triglycerides, $\mathrm{mmol} / \mathrm{L}$ & $R=-0.038$ & $R=-0.179$ & $R=-0.140$ & $\mathrm{R}=0.217$ \\
\hline Free fatty acids, $\mathrm{mmol} / \mathrm{L}$ & $R=-0.104$ & $R=-0.162$ & $R=-0.029$ & $R=-0.186$ \\
\hline Fasting glucose, $\mathrm{mmol} / \mathrm{L}$ & $\mathrm{R}=0.135^{\mathrm{A}}$ & $R=-0.089$ & $R=0.095$ & $R=-0.044$ \\
\hline 2-hour glucose, $\mathrm{mmol} / \mathrm{L}$ & $R=-0.021$ & $R=-0.049$ & $R=0.026$ & $R=-0.098$ \\
\hline Fasting insulin, $\mu U / L$ & $R=0.037$ & $\mathrm{R}=0.110$ & $R=-0.132$ & $R=0.082$ \\
\hline HOMA-IR & $R=0.045$ & $\mathrm{R}=0.052$ & $R=-0.097$ & $R=0.052$ \\
\hline Systolic bloodpressure z-score & $R=-0.097$ & $R=-0.091$ & $R=0.019$ & $R=-0.187$ \\
\hline Diastolic bloodpressure z-score & $R=-0.082$ & $R=-0.036$ & $R=-0.116$ & $R=-0.166$ \\
\hline $\mathrm{CRP}, \mathrm{mg} / \mathrm{L}$ & $R=-0.193^{A}$ & $R=0.027$ & $R=0.072$ & $R=-0.198$ \\
\hline
\end{tabular}

$A=$ significant correlation between the hepatorenal index and other health parameters

Table 3. Regressionmodel for predictors of logHRI

\begin{tabular}{|c|c|c|c|c|}
\hline & \multicolumn{2}{|c|}{ Total population $(n=258)$} & \multicolumn{2}{|c|}{ Children with severe hepatic steatosis $(n=84)$} \\
\hline & $\beta$ & p-value & $\beta$ & p-value \\
\hline & $R^{2}=0.263$ & & $\mathrm{R}^{2}=0.320$ & \\
\hline$A L T, U / L$ & 0.003 & $<0.001$ & 0.002 & 0.001 \\
\hline Sex, male & 0.020 & 0.197 & 0.016 & 0.491 \\
\hline BMI z-score & -0.018 & 0.106 & -0.006 & 0.692 \\
\hline Age, years & -0.007 & 0.001 & -0.002 & 0.484 \\
\hline$C R P, \mathrm{mg} / \mathrm{L}$ & -0.002 & 0.042 & -0.008 & 0.067 \\
\hline Fasting glucose, $\mathrm{mmol} / \mathrm{L}$ & 0.025 & 0.039 & 0.010 & 0.535 \\
\hline Ferritin, $\mu \mathrm{g} / \mathrm{L}$ & - & - & 0.000 & 0.699 \\
\hline
\end{tabular}

B's shown are unstandardised.

Differences between children with ALT concentrations below and above the upper limit of normal

Children with ALT concentrations above the ULN had a significantly higher HRI than children with ALT concentrations below the ULN (respectively, $1.49(1.29 ; 1.88)$ vs. 1.45 $(1.25 ; 1.63) ; p=0.021)$. They also had higher ferritin concentrations $(52.0(33.0 ; 82.3)$ vs. $42.0(31.0 ; 58.8) ; p=0.005)$, lower HDL cholesterol concentrations $(1.20(1.10 ; 1.40)$ vs 1.30 $(1.10 ; 1.53) ; p=0.010)$, higher triglyceride concentrations $(0.97(0.72 ; 1.47)$ vs. 0.83 
(0.61;1.08); $p=0.001)$, lower fasting glucose concentrations ( $4.39 \pm 0.55$ vs. $4.60 \pm 0.61$; $\mathrm{p}=0.008)$, higher fasting insulin concentrations $(16.83(9.21 ; 26.63)$ vs. 12.53 (7.71;18.42); $\mathrm{p}=0.008)$ and a higher HOMA-IR $(3.16(1.73 ; 5.11)$ vs. $2.52(1.47 ; 3.86) ; \mathrm{p}=0.027)$ (table 4).

\section{Determinants of steatohepatitis}

In the whole group, ALT concentrations correlated with BMI z-score ( $R=0.290), H R I$ $(R=0.225)$, AST concentrations $(R=0.589), \quad G G T$ concentrations $(R=0.513)$, alkaline phosphatase concentrations ( $R=0.128)$, ferritin concentrations $(R=0.282)$, HDL cholesterol concentrations $(R=-0.165)$, triglyceride concentrations $(R=0.192)$, fasting insulin concentrations ( $R=0.196)$ and HOMA-IR $(R=0.185)$ (table 5).

Regression analysis identified HRI $(\beta=0.165, p<0.001)$ as a significant determinant of $\log A L T$ concentrations. Additionally, sex $(\beta=0.068, p=0.015)$, BMI z-score $(\beta=0.059$; $p=0.004)$, triglyceride concentrations $(\beta=0.073, p=0.006)$ and ferritin concentrations $(\beta=0.001, p=0.036)$ were significantly associated with $\log A L T$ concentrations $\left(R^{2}=0.324\right)$. There was a trend towards a contribution of age $(\beta=0.008, p=0.051)$ (table 6$)$.

In the children with severe hepatic steatosis, HRI $(\beta=0.188, p<0.001)$, fasting insulin concentrations $(\beta=-0.002, p=0.026)$, triglyceride concentrations $(\beta=0.087, p=0.037)$ and ferritin concentrations $(\beta=0.003, p<0.001)$ were significantly associated with $\operatorname{logALT}$ concentrations $\left(R^{2}=0.563\right)$, as a measure of steatohepatitis (table 6$)$. Within this subgroup, 13 children had ALT concentrations higher than twice the upper limit of normal, of which 12 were boys. 


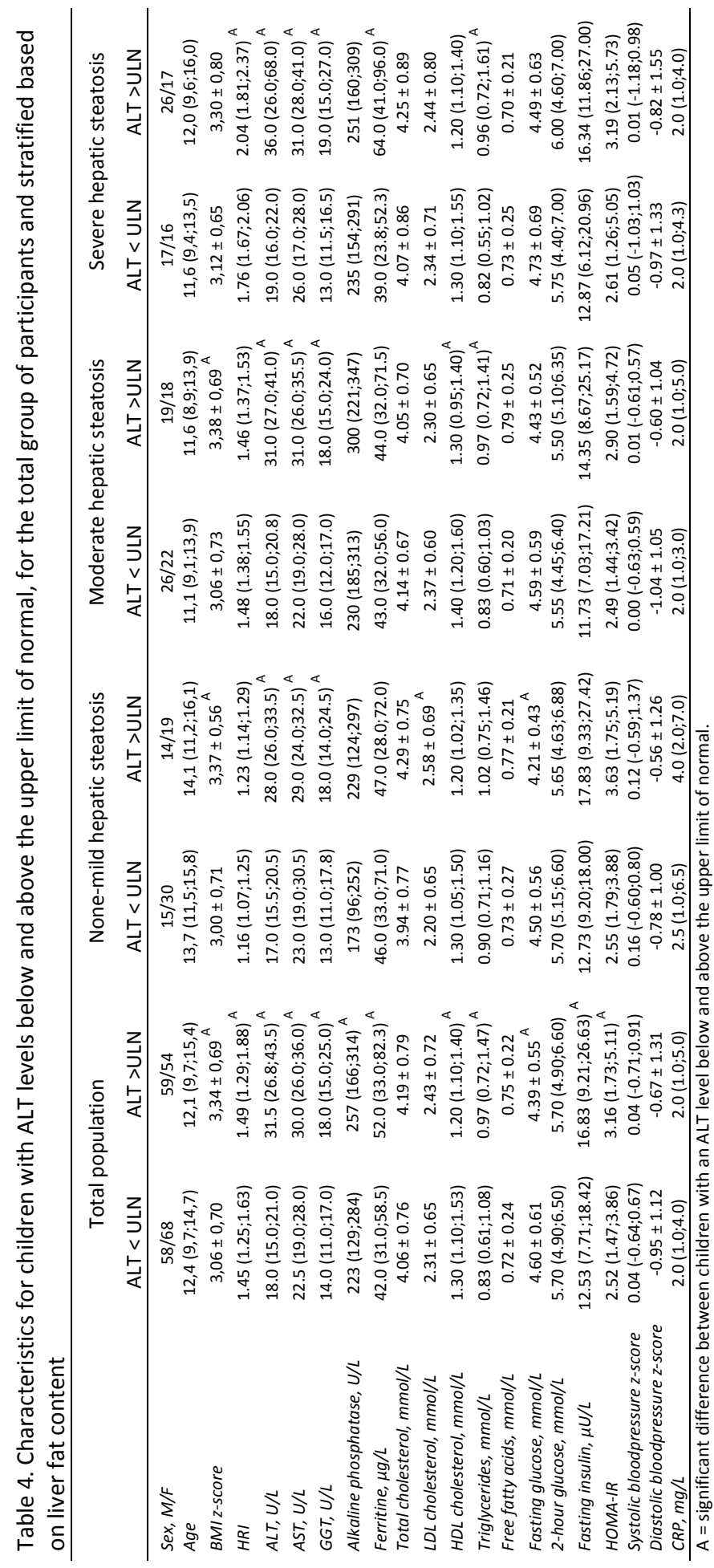


Table 5. Correlation coefficients between ALT and health parameters, in the total population and stratified based on liver fat content

\begin{tabular}{|c|c|c|c|c|}
\hline & $\begin{array}{c}\text { Total } \\
\text { population }\end{array}$ & $\begin{array}{l}\text { None-mild hepatic } \\
\text { steatosis }\end{array}$ & $\begin{array}{l}\text { Moderate hepatic } \\
\text { steatosis }\end{array}$ & $\begin{array}{l}\text { Severe hepatic } \\
\text { steatosis }\end{array}$ \\
\hline$H R I$ & $R=0.225^{A}$ & $R=0.110$ & $R=0.063$ & $R=0.543^{A}$ \\
\hline Age, years & $R=0.034$ & $R=-0.016$ & $R=0.059$ & $R=0.117$ \\
\hline BMI z-score & $\mathrm{R}=0.290^{\mathrm{A}}$ & $R=0.396^{A}$ & $R=0.302^{A}$ & $R=0.216$ \\
\hline$A S T, U / L$ & $R=0.589^{A}$ & $R=0.426^{A}$ & $R=0.599^{A}$ & $\mathrm{R}=0.701^{\mathrm{A}}$ \\
\hline$G G T, U / L$ & $R=0.513^{A}$ & $R=0.507^{A}$ & $\mathrm{R}=0.354^{\mathrm{A}}$ & $\mathrm{R}=0.675^{\mathrm{A}}$ \\
\hline Alkaline phosphatase, $U / L$ & $R=0.128^{A}$ & $R=0.166$ & $R=0.139$ & $R=0.044$ \\
\hline Ferritin, $\mu g / L$ & $\mathrm{R}=0.282^{\mathrm{A}}$ & $R=0.104$ & $R=0.210$ & $\mathrm{R}=0.546^{\mathrm{A}}$ \\
\hline Total cholesterol, $\mathrm{mmol} / \mathrm{L}$ & $R=0.096$ & $R=0.213$ & $R=-0.027$ & $R=0.115$ \\
\hline LDL cholesterol, $\mathrm{mmol} / \mathrm{L}$ & $R=0.092$ & $\mathrm{R}=0.270^{\mathrm{A}}$ & $R=-0.026$ & $R=0.060$ \\
\hline HDL cholesterol, $\mathrm{mmol} / \mathrm{L}$ & $R=-0.165^{A}$ & $R=-0.147$ & $R=-0.230^{A}$ & $R=-0.132$ \\
\hline Triglycerides, $\mathrm{mmol} / \mathrm{L}$ & $\mathrm{R}=0.192^{\mathrm{A}}$ & $R=0.142$ & $R=0.239^{A}$ & $R=0.294^{A}$ \\
\hline Free fatty acids, $\mathrm{mmol} / \mathrm{L}$ & $R=0.016$ & $R=0.038$ & $R=0.090$ & $R=-0.053$ \\
\hline Fasting glucose, $\mathrm{mmol} / \mathrm{L}$ & $R=-0.050$ & $R=-0.151$ & $R=-0.024$ & $R=-0.056$ \\
\hline 2-hour glucose, $\mathrm{mmol} / \mathrm{L}$ & $R=0.047$ & $R=-0.001$ & $\mathrm{R}=0.018$ & $R=0.048$ \\
\hline Fasting insulin, $\mu U / L$ & $R=0.196^{A}$ & $R=0.148$ & $\mathrm{R}=0.251^{\mathrm{A}}$ & $R=0.219$ \\
\hline HOMA-IR & $\mathrm{R}=0.185^{\mathrm{A}}$ & $\mathrm{R}=0.138$ & $R=0.263^{A}$ & $\mathrm{R}=0.194$ \\
\hline Systolic bloodpressure z-score & $R=-0.019$ & $R=0.105$ & $R=0.066$ & $R=-0.191$ \\
\hline Diastolic bloodpressure z-score & $R=0.050$ & $R=0.011$ & $\mathrm{R}=0.234$ & $R=-0.055$ \\
\hline $\mathrm{CRP}, \mathrm{mg} / \mathrm{L}$ & $R=0.052$ & $R=0.154$ & $R=0.067$ & $\mathrm{R}=0.028$ \\
\hline
\end{tabular}

$A=$ significant correlation between ALT concentrations and other health parameters

Table 6. Regressionmodel for predictors of $\log A L T$

\begin{tabular}{|c|c|c|c|c|}
\hline & \multicolumn{2}{|c|}{ Total population $(n=258)$} & \multicolumn{2}{|c|}{$\begin{array}{c}\text { Children with severe hepatic } \\
\text { steatosis }(n=84)\end{array}$} \\
\hline & $\beta$ & p-value & $\beta$ & $p$-value \\
\hline & $\mathrm{R}^{2}=0.32$ & & $R^{2}=0.563$ & \\
\hline$H R I$ & 0.165 & $<0.001$ & 0.188 & $<0.001$ \\
\hline Sex, male & 0.068 & 0.015 & 0.082 & 0.124 \\
\hline Age, years & 0.008 & 0.051 & 0.001 & 0.885 \\
\hline BMI z-score & 0.059 & 0.004 & 0.044 & 0.220 \\
\hline Triglycerides, $\mathrm{mmol} / \mathrm{L}$ & 0.073 & 0.006 & 0.087 & 0.037 \\
\hline Ferritin, $\mu g / L$ & 0.001 & 0.036 & 0.003 & $<0.001$ \\
\hline HDL cholesterol, $\mathrm{mmol} / \mathrm{L}$ & -0.001 & 0.978 & - & - \\
\hline HOMA & -0.004 & 0.234 & - & - \\
\hline Fasting insulin, $\mu U / L$ & - & - & -0.002 & 0.026 \\
\hline
\end{tabular}




\section{Discussion}

In this study we identified various factors associated with increasing degrees of hepatic steatosis, as measured with the ultrasonographic hepatorenal index, and subsequently identified factors associated with progression to steatohepatitis, as assessed by alanine aminotransferase level, in children with overweight, obesity or morbid obesity. The results of this study demonstrated that the factors related to development of steatosis in children are not the same as the factors associated with progression to steatohepatitis in children. A summary of these factors is shown in Figure 1.

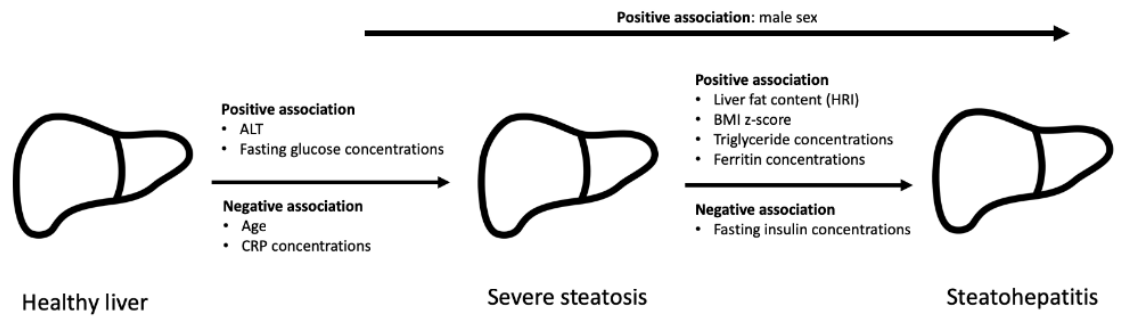

Figure 1. A summary of the factors that were associated with hepatic steatosis and steatohepatitis in this study

Fasting glucose concentrations, age and CRP concentrations were associated with an increasing liver fat content. The association between the HRI and fasting glucose concentrations is in line with a previous study in adults evaluating the association between metabolic parameters and HRI. Other studies in children and adults have also described higher fasting glucose concentrations in patients with hepatic steatosis diagnosed with qualitative ultrasonography $(29,30)$.

We found an inverse association between HRI and age, reflecting a higher amount of liver fat in younger children in our cohort. Separate correlation analysis for sex showed that the inverse association between the HRI and age in the total group of children was driven by an inverse association in girls, but not boys. Previously, Denzer et al. also found that the prevalence of NAFLD decreased in girls from puberty onwards (31). Furthermore, it is possible that there was some degree of selection bias in our cohort, since the children that were referred to our obesity expertise center at a young age, might have developed (morbid) obesity earlier than children that are referred at a later age. These children that develop obesity already at a very young age might also have a different (genetic) risk profile, possibly also contributing to an increased risk of NAFLD. 
Remarkably, our results also showed a negative association between the HRI and CRP. Multiple studies have shown higher CRP concentrations in children and adults with NAFLD $(32,33)$. However, a large adult study has described an association with NAFLD development in adults with a high CRP (i.e. the upper quartile), but also in adults with a low CRP (i.e. the lowest quartile), suggesting that a certain concentration of CRP might even be protective against NAFLD (34). Additional studies are needed to replicate these findings and further elucidate how different CRP concentrations are associated with NAFLD.

Interestingly, we did not find an association between the hepatorenal index and BMI zscore or metabolic aberrations, apart from the association with fasting glucose concentrations. Studies evaluating associations between cardiometabolic and anthropometric parameters with liver fat content as estimated with the HRI are scarce, even in adults, and have to the best of our knowledge not been described in children so far. In a previous study in adults, Xia et al. found a significant correlation between quantitative ultrasound measurement of fat content and BMI, waist-circumference, triglyceride concentrations, HDL cholesterol concentrations, fasting glucose concentrations and OGTT 2-hour glucose concentrations (35). It is important to note that in that study, hepatic fat content was estimated by a combined score of the hepatorenal index and the hepatic attenuation rate, rather than only the HRI. However, the authors also showed that the addition of the hepatic attenuation rate made a very minimal difference in the prediction of fat content by HRI compared to ${ }^{1} \mathrm{H}-\mathrm{MRS}$. Similarly, Zhang et al. found a correlation between triglyceride concentrations and HDL cholesterol concentrations and a combined score of the hepatorenal ratio and hepatic attenuation rate (19). Both studies were performed in a Chinese population, that was not limited to people with overweight and obesity and included people from a wide age range (16-75 years). Since ethnicity and age have both been associated with NAFLD, these population differences might contribute to differences in results between these studies and our study. Since not all participants had overweight or obesity in these adult studies, in contrast to our study, a positive correlation between BMI and liver fat content could also suggest that overweight or obesity contributes to the presence of hepatic steatosis, but a higher degree of overweight does not necessarily lead to a higher liver fat content.

The absence of a correlation between the HRI and metabolic parameters might also be a reflection of the fact that the direction of the relation between NAFLD and metabolic aberrations remains to be further clarified. Even though NAFLD is often associated with (components of) the metabolic syndrome, it is not yet clear if NAFLD precedes the development of metabolic aberrations or vice versa, or if the association is bidirectional 
(36). In the scenario that hepatic steatosis is the starting point, it is possible that the development of metabolic aberrations is still limited in our pediatric population. Additional studies are needed to further clarify this.

Finally, the fact that we were able to predict $26 \%$ of HRI variance with the parameters in our regression model demonstrates that other parameters are important additional predictors of liver fat content. In recent years, genetic studies have shown a strong contribution of several single nucleotide polymorphisms (SNPs), to the development of NAFLD. Of these SNPs, the PNPLA3 I148M and TM6SF2 E167K variants are the most wellknown at this time, however several more SNPs that contribute to NAFLD development and progression have been identified. A previous study in adult twins showed that, after adjustment for age, sex and ethnicity, the heritability of hepatic steatosis was 0.52 (37), showing that genetic predisposition plays a very important role. Additionally, dietary habits might also play role in the development of fatty liver disease. For example, fructose intake has been shown to increase de novo lipogenesis (38), thereby contributing to the development of hepatic steatosis $(38,39)$. Additionally, a low vitamin $E$ intake has been associated with steatosis severity in children (40). In this study, we did not take diet into account.

Our results further showed that a higher degree of hepatic steatosis was an important determinant of ALT concentrations. Additionally, male sex, higher BMI z-score, and higher triglyceride and ferritin concentrations were all associated with elevated ALT concentrations. Besides ferritin and triglyceride concentrations, lower fasting insulin concentrations were associated with higher ALT concentrations, in the children with severe hepatic steatosis. In this subgroup, the associated factors explained in total $56 \%$ of ALT variance.

In accordance with our study, previous studies have also shown that steatohepatitis is more prevalent in males $(41,42)$. This sex difference is suggested to be, at least partly, due to a protective effect of estrogens, as well as differences in body fat distribution, metabolic processes in the liver and in immune responses (14).

Contrary to our findings regarding the hepatorenal index, we did find an association between BMI z-score and ALT concentrations. However, this association was not seen in the children with a high liver fat content, suggesting that increasing concentrations of obesity are less important in predicting a worse outcome in children that already have a fatty liver.

The association between serum triglyceride concentrations and steatohepatitis in our study is in agreement with previous studies in children and adults $(43,44)$. Kong et al. also 
found higher triglyceride concentrations in children with ALT concentrations above the $75^{\text {th }}$ percentile (43). Estimates of liver fat content were not taken into account in that study. Kashyap et al. found slightly elevated triglyceride concentrations in morbidly obese adults with biopsy-proven hepatic steatosis compared to adults without steatosis, but a strong elevation of triglyceride concentrations in patients with NASH (44). Similarly, Junior et al. found that serum triglyceride concentrations were not elevated in morbidly obese women with steatosis, but only in women with $\mathrm{NASH}$, compared to women without NAFLD (45). Combined with our results, these findings might suggest that the association between triglyceride concentrations and NAFLD becomes relevant during the progression to steatohepatitis, but not yet in the development of hepatic steatosis.

Serum ferritin concentrations were also significantly associated with ALT concentrations in our study. In a meta-analysis of 14 studies, Du et al. also showed that serum ferritin concentrations were elevated in patients with NAFLD, compared to controls. Moreover, ferritin concentrations were higher in patients with steatohepatitis compared to patients with steatosis (46), which is in line with our findings. The association between NAFLD and serum ferritin levels is not yet fully elucidated. Previous studies have suggested that elevated serum ferritin levels may reflect mild iron deposition in the liver (47), since ferritin is the main storage protein of iron in the liver. Hepatic iron concentrations have been associated with oxidative stress, production of pro-inflammatory cytokines (48), and insulin resistance (47). Moreover, ferritin itself has also been shown to act as a proinflammatory cytokine, contributing to activation of hepatic stellate cells (47).

Finally, we found an inverse association between fasting insulin concentrations and ALT concentrations in the children with a high amount of liver fat. This is a remarkable finding, especially since previous studies have shown a positive association between insulin resistance and NAFLD (6). In our study, the inverse association was limited to the regression analysis in children with severe hepatic steatosis. However, in the correlation analysis, there was a positive association between ALT concentrations and fasting insulin concentrations in the whole group and a trend towards a positive association in the children with a high liver fat content. It could be that the inverse association that we found in the regression analysis is a consequence of an association with other covariates in the model. For instance, previous studies and our own data showed a correlation between insulin and ferritin concentrations (even though we did not find multicollinearity in our model). A similar regression model (data not shown) without ferritin concentrations, did not show a significant association between ALT and fasting insulin concentrations. Although not fully understood, this observation might suggest that there 
is a connection between ferritin and insulin concentrations in explaining variation in ALT concentrations in children with high HRI values.

Currently, the factors associated with (fast) progression through the different stages of NAFLD disease severity are incompletely understood. The findings of our study demonstrate that the NAFLD spectrum cannot be seen as one continuum in regard to underlying pathophysiological processes, as we have shown that the determinants of hepatic steatosis in children with overweight and obesity are not the same as the factors that are associated with the progression from steatosis to steatohepatitis. High ALT concentrations were especially common in boys with a high liver fat content, a high BMI zscore, higher serum triglyceride concentrations and higher serum ferritin concentrations. Further studies are needed to determine reference values for the hepatorenal index in children and to determine how other factors, like genetic predisposition and dietary composition, contribute to the factors that were already identified in this study. As we have only studied factors that are associated with hepatic steatosis and steatohepatitis in cross-sectional data, longitudinal studies are helpful to further validate and extend these findings. Long-term follow-up studies will give more insight into the pace of progression through the NAFLD disease spectrum in children and how this might be influenced by the associated factors.

\section{Conclusion}

In children with overweight, obesity and morbid obesity, age, fasting glucose and CRP are associated with an increasing degree of hepatic steatosis. A higher degree of hepatic steatosis is associated with progression to steatohepatitis, just like the severity of overweight, male sex, serum triglyceride concentrations and serum ferritin concentrations. These results provide important insight into the factors that are associated with the consecutive NAFLD stages in children with overweight and obesity and demonstrate that it is important to separately study the different stages of NAFLD and the associated pathophysiological factors, in order to gain a better understanding of the progression along the disease spectrum. 


\section{References}

1. Marengo A, Jouness RI, Bugianesi E. Progression and Natural History of Nonalcoholic Fatty Liver Disease in Adults. Clinics in liver disease. 2016;20(2):313-24.

2. White DL, Kanwal F, El-Serag HB. Association between nonalcoholic fatty liver disease and risk for hepatocellular cancer, based on systematic review. Clinical gastroenterology and hepatology : the official clinical practice journal of the American Gastroenterological Association. 2012;10(12):1342-59.e2.

3. Valenti L, Bugianesi E, Pajvani U, Targher G. Nonalcoholic fatty liver disease: cause or consequence of type 2 diabetes? Liver international : official journal of the International Association for the Study of the Liver. 2016;36(11):1563-79.

4. Sookoian S, Pirola CJ. Non-alcoholic fatty liver disease is strongly associated with carotid atherosclerosis: a systematic review. Journal of hepatology. 2008;49(4):600-7.

5. Anderson EL, Howe LD, Jones HE, Higgins JP, Lawlor DA, Fraser A. The Prevalence of NonAlcoholic Fatty Liver Disease in Children and Adolescents: A Systematic Review and MetaAnalysis. PloS one. 2015;10(10):e0140908.

6. Prokopowicz Z, Malecka-Tendera E, Matusik P. Predictive Value of Adiposity Level, Metabolic Syndrome, and Insulin Resistance for the Risk of Nonalcoholic Fatty Liver Disease Diagnosis in Obese Children. Canadian journal of gastroenterology \& hepatology. 2018;2018:9465784.

7. Kanwar P, Kowdley KV. The Metabolic Syndrome and Its Influence on Nonalcoholic Steatohepatitis. Clinics in liver disease. 2016;20(2):225-43.

8. Romeo S, Kozlitina J, Xing C, Pertsemlidis A, Cox D, Pennacchio LA, et al. Genetic variation in PNPLA3 confers susceptibility to nonalcoholic fatty liver disease. Nature genetics. 2008;40(12):1461-5.

9. Chen LZ, Xia HH, Xin YN, Lin ZH, Xuan SY. TM6SF2 E167K Variant, a Novel Genetic Susceptibility Variant, Contributing to Nonalcoholic Fatty Liver Disease. Journal of clinical and translational hepatology. 2015;3(4):265-70.

10. Dai X, Wang B. Role of gut barrier function in the pathogenesis of nonalcoholic Fatty liver disease. Gastroenterology research and practice. 2015;2015:287348.

11. Mosca A, Nobili V, De Vito R, Crudele A, Scorletti E, Villani A, et al. Serum uric acid concentrations and fructose consumption are independently associated with NASH in children and adolescents. Journal of hepatology. 2017;66(5):1031-6.

12. Jensen T, Abdelmalek MF, Sullivan S, Nadeau KJ, Green M, Roncal C, et al. Fructose and sugar: A major mediator of non-alcoholic fatty liver disease. Journal of hepatology. 2018;68(5):1063-75.

13. Nati M, Haddad D, Birkenfeld AL, Koch CA, Chavakis T, Chatzigeorgiou A. The role of immune cells in metabolism-related liver inflammation and development of non-alcoholic steatohepatitis (NASH). Reviews in endocrine \& metabolic disorders. 2016;17(1):29-39.

14. Lonardo A, Nascimbeni F, Ballestri S, Fairweather D, Win S, Than TA, et al. Sex Differences in NAFLD: State of the Art and Identification of Research Gaps. Hepatology (Baltimore, Md). 2019.

15. Younossi ZM, Koenig AB, Abdelatif D, Fazel Y, Henry L, Wymer M. Global epidemiology of nonalcoholic fatty liver disease-Meta-analytic assessment of prevalence, incidence, and outcomes. Hepatology (Baltimore, Md). 2016;64(1):73-84.

16. Di Martino M, Pacifico L, Bezzi M, Di Miscio R, Sacconi B, Chiesa C, et al. Comparison of magnetic resonance spectroscopy, proton density fat fraction and histological analysis in the quantification of liver steatosis in children and adolescents. World journal of gastroenterology. 2016;22(39):8812-9. 
17. Webb M, Yeshua H, Zelber-Sagi S, Santo E, Brazowski E, Halpern Z, et al. Diagnostic value of a computerized hepatorenal index for sonographic quantification of liver steatosis. AJR American journal of roentgenology. 2009;192(4):909-14.

18. Marshall RH, Eissa M, Bluth El, Gulotta PM, Davis NK. Hepatorenal index as an accurate, simple, and effective tool in screening for steatosis. AJR American journal of roentgenology. 2012;199(5):997-1002.

19. Zhang B, Ding F, Chen T, Xia LH, Qian J, Lv GY. Ultrasound hepatic/renal ratio and hepatic attenuation rate for quantifying liver fat content. World journal of gastroenterology. 2014;20(47):17985-92.

20. Borges VF, Diniz AL, Cotrim HP, Rocha HL, Andrade NB. Sonographic hepatorenal ratio: a noninvasive method to diagnose nonalcoholic steatosis. Journal of clinical ultrasound : JCU. 2013;41(1):18-25.

21. Chauhan A, Sultan LR, Furth EE, Jones LP, Khungar V, Sehgal CM. Diagnostic accuracy of hepatorenal index in the detection and grading of hepatic steatosis. Journal of clinical ultrasound : JCU. 2016;44(9):580-6.

22. Senior JR. Alanine aminotransferase: a clinical and regulatory tool for detecting liver injurypast, present, and future. Clinical pharmacology and therapeutics. 2012;92(3):332-9.

23. Vos MB, Abrams SH, Barlow SE, Caprio S, Daniels SR, Kohli R, et al. NASPGHAN Clinical Practice Guideline for the Diagnosis and Treatment of Nonalcoholic Fatty Liver Disease in Children: Recommendations from the Expert Committee on NAFLD (ECON) and the North American Society of Pediatric Gastroenterology, Hepatology and Nutrition (NASPGHAN). Journal of pediatric gastroenterology and nutrition. 2017;64(2):319-34.

24. Verhaegh P, Bavalia R, Winkens B, Masclee A, Jonkers D, Koek G. Noninvasive Tests Do Not Accurately Nonalcoholic Steatohepatitis From Simple Steatosis: A Systematic Review and Meta-analysis. Clinical gastroenterology and hepatology : the official clinical practice journal of the American Gastroenterological Association. 2018;16(6):837-61.

25. Rijks JM, Plat J, Mensink RP, Dorenbos E, Buurman WA, Vreugdenhil AC. Children With Morbid Obesity Benefit Equally as Children With Overweight and Obesity From an Ongoing Care Program. The Journal of clinical endocrinology and metabolism. 2015;100(9):3572-80.

26. Schwimmer JB, Dunn W, Norman GJ, Pardee PE, Middleton MS, Kerkar N, et al. SAFETY study: alanine aminotransferase cutoff values are set too high for reliable detection of pediatric chronic liver disease. Gastroenterology. 2010;138(4):1357-64, 64.e1-2.

27. Friedewald WT, Levy RI, Fredrickson DS. Estimation of the concentration of low-density lipoprotein cholesterol in plasma, without use of the preparative ultracentrifuge. Clinical chemistry. 1972;18(6):499-502.

28. Wuhl E, Witte K, Soergel M, Mehls O, Schaefer F. Distribution of 24-h ambulatory blood pressure in children: normalized reference values and role of body dimensions. Journal of hypertension. 2002;20(10):1995-2007.

29. Bedogni G, Gastaldelli A, Manco M, De Col A, Agosti F, Tiribelli C, et al. Relationship between fatty liver and glucose metabolism: a cross-sectional study in 571 obese children. Nutrition, metabolism, and cardiovascular diseases : NMCD. 2012;22(2):120-6.

30. Lu ZY, Shao Z, Li YL, Wulasihan M, Chen XH. Prevalence of and risk factors for non-alcoholic fatty liver disease in a Chinese population: An 8-year follow-up study. World journal of gastroenterology. 2016;22(13):3663-9.

31. Denzer C, Thiere D, Muche R, Koenig W, Mayer H, Kratzer W, et al. Gender-specific prevalences of fatty liver in obese children and adolescents: roles of body fat distribution, sex steroids, and insulin resistance. The Journal of clinical endocrinology and metabolism. 2009;94(10):3872-81.

32. Kitsios K, Papadopoulou M, Kosta K, Kadoglou N, Papagianni M, Tsiroukidou K. Highsensitivity C-reactive protein levels and metabolic disorders in obese and overweight 
children and adolescents. Journal of clinical research in pediatric endocrinology. 2013;5(1):44-9.

33. Zimmermann E, Anty R, Tordjman J, Verrijken A, Gual P, Tran A, et al. C-reactive protein levels in relation to various features of non-alcoholic fatty liver disease among obese patients. Journal of hepatology. 2011;55(3):660-5.

34. Wang LR, Liu WY, Wu SJ, Zhu GQ, Lin YQ, Braddock M, et al. Parabolic relationship between sex-specific serum high sensitive $C$ reactive protein and non-alcoholic fatty liver disease in Chinese adults: a large population-based study. Oncotarget. 2016;7(12):14241-50.

35. Xia MF, Yan HM, He WY, Li XM, Li CL, Yao XZ, et al. Standardized ultrasound hepatic/renal ratio and hepatic attenuation rate to quantify liver fat content: an improvement method. Obesity (Silver Spring, Md). 2012;20(2):444-52.

36. Wainwright $P$, Byrne CD. Bidirectional Relationships and Disconnects between NAFLD and Features of the Metabolic Syndrome. International journal of molecular sciences. 2016;17(3):367.

37. Loomba R, Schork N, Chen CH, Bettencourt R, Bhatt A, Ang B, et al. Heritability of Hepatic Fibrosis and Steatosis Based on a Prospective Twin Study. Gastroenterology. 2015;149(7):1784-93.

38. Schwarz JM, Noworolski SM, Wen MJ, Dyachenko A, Prior JL, Weinberg ME, et al. Effect of a High-Fructose Weight-Maintaining Diet on Lipogenesis and Liver Fat. The Journal of clinical endocrinology and metabolism. 2015;100(6):2434-42.

39. Volynets V, Machann J, Kuper MA, Maier IB, Spruss A, Konigsrainer A, et al. A moderate weight reduction through dietary intervention decreases hepatic fat content in patients with non-alcoholic fatty liver disease (NAFLD): a pilot study. European journal of nutrition. 2013;52(2):527-35.

40. Vos MB, Colvin R, Belt P, Molleston JP, Murray KF, Rosenthal P, et al. Correlation of vitamin $E$, uric acid, and diet composition with histologic features of pediatric NAFLD. Journal of pediatric gastroenterology and nutrition. 2012;54(1):90-6.

41. Wang Z, Xu M, Hu Z, Hultstrom M, Lai E. Sex-specific prevalence of fatty liver disease and associated metabolic factors in Wuhan, south central China. European journal of gastroenterology \& hepatology. 2014;26(9):1015-21.

42. Long MT, Pedley A, Massaro JM, Hoffmann U, Ma J, Loomba R, et al. A simple clinical model predicts incident hepatic steatosis in a community-based cohort: The Framingham Heart Study. Liver international : official journal of the International Association for the Study of the Liver. 2018;38(8):1495-503.

43. Kong AP, Choi KC, Cockram CS, Ho CS, Chan MH, Ozaki R, et al. Independent associations of alanine aminotransferase (ALT) levels with cardiovascular risk factor clustering in Chinese adolescents. Journal of hepatology. 2008;49(1):115-22.

44. Kashyap SR, Diab DL, Baker AR, Yerian L, Bajaj H, Gray-McGuire C, et al. Triglyceride levels and not adipokine concentrations are closely related to severity of nonalcoholic fatty liver disease in an obesity surgery cohort. Obesity (Silver Spring, Md). 2009;17(9):1696-701.

45. Junior WS, Nonino-Borges CB. Clinical predictors of different grades of nonalcoholic fatty liver disease. Obesity surgery. 2012;22(2):248-52.

46. Du SX, Lu LL, Geng N, Victor DW, Chen LZ, Wang C, et al. Association of serum ferritin with non-alcoholic fatty liver disease: a meta-analysis. Lipids in health and disease. 2017;16(1):228.

47. Lombardi R, Pisano G, Fargion S. Role of Serum Uric Acid and Ferritin in the Development and Progression of NAFLD. International journal of molecular sciences. 2016;17(4):548.

48. Britton LJ, Subramaniam VN, Crawford DH. Iron and non-alcoholic fatty liver disease. World journal of gastroenterology. 2016;22(36):8112-22. 


\section{Chapter 7}

Genotype-specific determinants of alanine aminotransferase levels in children with overweight and obesity

Kylie Karnebeek

Jogchum Plat

Anita C.E. Vreugdenhil

Submitted 


\section{Abstract}

\section{Importance}

Non-alcoholic fatty liver disease (NAFLD) is a health threat in children with obesity. Several factors associated with development of NAFLD have been identified, including obesity, metabolic aberrations and sex. Also, SNPs in several genes predispose to the development of NAFLD. Since it concerns variants in genes encoding proteins involved in different processes of NAFLD development, it can be hypothesized that factors associated with NAFLD might be genotype and SNP specific.

\section{Objective}

To identify determinants of alanine aminotransferase (ALT), as a marker for NAFLD, in children with overweight and obesity, and to evaluate if these determinants differ depending on genetic variants.

\section{Setting}

This study was conducted within the Centre for Overweight Adolescent and Children's Healthcare $(\mathrm{COACH})$ at the Maastricht University Medical Centre.

\section{Participants}

One hundred and seventy-four children (49\% boys) with overweight, obesity or morbid obesity.

\section{Main outcome and measures}

PNPLA3 I148M, TM6SF2 E167K and GPR120 R270H genotypes, as well as liver-related, anthropometric and cardiometabolic parameters were determined.

\section{Results}

In the whole group, sex, total cholesterol levels, triglyceride (TG) levels, HOMA-IR and PNPLA3 and TM6SF2 risk alleles were associated with logALT levels. Stratification for genotypes showed that sex and HOMA-IR were associated significantly with logALT levels in children with and without the PNPLA3 risk allele. Interestingly, triglyceride levels were only associated with logALT levels in carriers of the PNPLA3 risk allele. The percentage of children with ALT levels above normal was significantly higher in PNPLA3 risk allele carriers compared to non-carriers, while the percentage of children with triglyceride levels above normal did not differ. 
Sex, total cholesterol levels, triglyceride levels and HOMA-IR were associated with logALT in TM6SF2 non-carriers, but not in carriers of the TM6SF2 risk allele.

\section{Conclusion and relevance}

Determinants of ALT levels are SNP and genotype-specific in children with overweight. Furthermore, high ALT levels can be found in children with overweight and a genetic predisposition, even when genotype-specific determinants of ALT levels such as TG levels in PNPLA3 risk allele carriers, are within the normal range. 


\section{Introduction}

Non-alcoholic fatty liver disease (NAFLD) has developed to be one of the most common chronic liver diseases, most likely as a consequence of the obesity epidemic (1). Given the increasing prevalence of obesity during childhood, the development of obesity-related liver disease at a young age has become an urgent topic. Especially since fatty liver disease can progress to liver cirrhosis and increases the risk of developing hepatocellular carcinoma at a later age (2), but also since NAFLD has been associated with the presence of several comorbidities, including diabetes type II (3) and atherosclerosis (4).

Previous studies have identified several predictors for the development of NAFLD, in addition to obesity, such as sex (5) and ethnicity (6). Also, NAFLD has been assumed to be the hepatic manifestation of the metabolic syndrome and was associated with insulin resistance and elevated triglyceride levels $(7,8)$. Recently, studies have also shown an important role for genetics in the development and progression of NAFLD (9). Genomewide association studies in adults have identified several single nucleotide polymorphisms (SNPS) that are associated with NAFLD. Interestingly, these genetic variants associated with NAFLD susceptibility are located in different genes and encode proteins involved in various processes. Firstly, the I148M-variant of the patatin-like phospholipase domain containing 3 (PNPLA3, rs738409 C/G) gene was identified as an important genetic determinant for the presence and severity of steatosis (10-12) and fibrosis (12). Increased liver fat content in PNPLA3 G allele carriers was already seen in children under the age of 10 years (13). The PNPLA3 gene encodes for the adiponutrin enzyme, which is primarily expressed in hepatocytes (14). It has been suggested that the I148M variant of PNPLA3 affects lipid droplet size (15) and leads to accumulation of hepatic triglycerides (16). More recently, additional genetic variants of genes that are associated with NAFLD have been identified, i.e. the E167K variant of the transmembrane 6 superfamily member 2 (TM6SF2, rs58542926 C/T). The presence of this minor allele reduces TM6SF2 function, leading to decreased very-low-density-lipoprotein secretion (17) and thereby increasing hepatic triglyceride content (HTC), but also improving the overall cardiovascular risk profile $(18,19)$.

A third SNP that has been associated with NAFLD is the R270H variant of the G-proteincoupled receptor 120 (GPR120, rs116454156 G/A). GPR120 is a receptor for polyunsaturated fatty acids (PUFAs) and normally modulates the anti-inflammatory effects of these PUFAs (20). However, these anti-inflammatory effects are impaired in the $\mathrm{R} 270 \mathrm{H}$ variant (21). Marzuillo et al. showed that this GPR120 variant is mainly associated with causing inflammation in livers that have already developed steatosis (21).

Previous studies aiming at finding factors that are involved in the development of hepatic steatosis or steatohepatitis have mainly focused on whole populations. With the new 
insight of the important contribution of specific SNPs in NAFLD susceptibility the question rises whether factors involved in NAFLD development depend on these genetic variants. Mechanisms underlying the development of NAFLD are likely to differ between these genetic variants, depending on the gene involved and the effects on expression and function of the respective protein. Therefore, the aim of this study is to identify determinants of ALT levels, as a marker for NAFLD, in a population of children with overweight and obesity, and to evaluate if these determinants differ depending on genetic variants.

\section{Methods}

\section{Participants}

This study was conducted within the Centre for Overweight Adolescent and Children's Healthcare $(\mathrm{COACH})$ at the Maastricht University Medical Centre. Children and adolescents with overweight, obesity and morbid obesity are evaluated, monitored and guided in this expertise centre as described previously (22). The study was conducted according to the guidelines of the Declaration of Helsinki and approved by the medical ethical committee of the MUMC+. Informed consent was obtained from the children and/or their parents.

\section{Analysis of polymorphisms}

Subjects were genotyped for single nucleotide polymorphisms in PNPLA3 rs738409, TM6SF2 rs58542926 and GPR120 rs116454156 using specific Taqman assays (Thermo Fisher Scientific).

\section{Anthropometric measurements}

Anthropometric measurements were performed while children were barefoot and wearing only underwear. Weight was measured on a digital scale (Seca) and height was measured using a digital stadiometer (De Grood Metaaltechniek). Body Mass Index (BMI) was calculated and BMI z-scores were obtained using a growth analyser (Growth Analyser VE). All children were classified as overweight, obese or morbidly obese based on International Obesity Task Force criteria (23). Waist circumference standard deviation scores were determined according to Dutch reference values (24). 


\section{Parameters reflecting liver health}

Serum alanine transaminase (ALT), aspartate transaminase (AST), gamma-glutamyl transferase, alkaline phosphatase and serum ferritin concentrations were determined with the Cobas 8000 modular analyser (Roche). For analyses in this study, the upper limit of normal (ULN) used for ALT was 22,1 U/L for girls and 25,8 U/L for boys based on a previous study examining the healthy range of ALT in children and adolescents (25). Possible causes of liver disease (infectious, autoimmune, toxic, endocrine and anatomic) were excluded in children with ALT values above $80 \mathrm{U} / \mathrm{L}$, excluding these diseases as possible confounding factors.

\section{Metabolic and cardiovascular parameters}

Fasting serum total cholesterol, high-density-lipoprotein (HDL) cholesterol, triglycerides (TG), free fatty acids and blood glucose were determined with the Cobas 8000 modular analyser and serum insulin levels with the Immulite 1000 (Siemens). Low-density lipoprotein (LDL) cholesterol was calculated using the Friedewald equation (26). Insulin resistance was evaluated by calculating the homeostasis model assessment for insulin resistance (HOMA-IR) (HOMA-IR = fasting glucose $(\mathrm{mmol} / \mathrm{L}) \times$ fasting insulin $(\mu \mathrm{U} / \mathrm{L}) / 22.5)$. Daytime blood pressure was measured approximately 20 times using the Mobil-O-Graph (I.E.M. GmbH). Blood pressure z-scores were calculated according to reference values for height and sex (27).

\section{Statistical analysis}

Statistical analyses were performed with IBM SPSS statistics 22.0. A chi-squared test was used to assess if genotypes were in Hardy-Weinberg equilibrium. Data was tested for normality with the Shapiro-Wilk test and reported as mean \pm standard deviation or median (interquartile range), as appropriate. Differences in parameters between genotype groups were analyzed with a Mann-Whitney $U$ test, ANOVA or Kruskal-Wallis test, as appropriate.

Multivariable regression analysis was performed to identify contributors to ALT levels. Possible contributors were identified from correlation analysis (significant correlations or trends). Due to multicollinearity HOMA-IR and fasting insulin levels were not both added to the regression analysis. The models were corrected for age and BMI z-score, since these variables correlated significantly with HOMA-IR. For the regression analyses, ALT levels were log-transformed to correct for a skewed distribution. 
A p-value $<0.05$ was considered statistically significant and was corrected for multiple testing if applicable.

\section{Results}

\section{Baseline characteristics}

One hundred and seventy-four children (49\% boys) were included. Forty \% of the children was heterozygote for the PNPLA3 G allele and 9\% was homozygote (GG). Fourteen \% was carrier of the TM6SF2 minor T allele, of which one child was homozygote TT. According to the dominant model, this child with the TT genotype was pooled with the heterozygotes for statistical analysis. Only three children were carrier of one GPR120 minor A allele (table 1). Therefore, further statistical analysis of GPR120 genotypes was limited. The frequency of the different genotypes was in Hardy-Weinberg equilibrium for all SNPS $(p>0,05)$.

\section{Associations between ALT levels and cardiometabolic parameters}

Correlation analysis showed that in the total group of participants ALT concentrations were positively associated with total cholesterol $(R=0.183, p=0.017)$, triglyceride levels $(R=0.213, p=0.005)$ and fasting insulin levels $(R=0.164, p=0.047)$ (table 2). There was a trend towards a significant correlation between ALT levels and HOMAIR $(R=0.153, p=0.065)$. Stratification for genotype showed that these three parameters did not correlate significantly with ALT in the PNPLA3 CC group, but that triglyceride levels, fasting insulin levels and HOMA-IR did correlate significantly with ALT in the CG group ( $R=0.317, r=0.275$ and $R=0.283$ respectively) and $G G$ group ( $R=0.750$, $R=0.674$ and $R=0.730$ respectively). ALT levels were positively correlated with total cholesterol and triglyceride levels in the TM6SF2 non-carriers $(R=0.206$ and $R=0.250$, respectively), but not in T allele carriers.

\section{Determinants of ALT levels}

Multivariable regression analysis identified $\operatorname{sex}(\beta=0.133, p<0.001)$, total cholesterol levels $(\beta=0.061, p=0.004)$, triglycerides levels $(\beta=0.064, p=0.008)$, HOMA-IR $(\beta=0.025, p=0.001)$ and both PNPLA3 (CG: $\beta=0.101, p=0.002 ; G G: \beta=0.203, p<0.001$ ) and TM6SF2 (CT\&TT: $\beta=0.124, p=0.008$ ) genotypes as significant predictors of logALT levels in the whole group (table 3). Stratification for genotype showed that in PNPLA3 risk allelecarriers (either CG 
or GG genotype), sex $(\beta=0.127, p=0.003)$, triglyceride levels $(\beta=0.108, p=0.009)$ and HOMA-IR ( $\beta=0.027, p=0.018$ ) were predictors of logALT levels (table 4). Also, there was a trend for a contribution of total cholesterol levels $(\beta=0.049, p=0.087)$. Interestingly, in non-carriers, only sex $(\beta=0.140, p=0.005)$ and HOMA-IR $(\beta=0.025, p=0.011)$, but not triglyceride levels, contributed significantly to the variation in $\log A L T$ levels.

An interaction term was added to the regression model (for the whole group), for either 'triglyceride levels * PNPLA3 CG or GG genotype', 'HOMA-IR * PNPLA3 CG or GG genotype' or 'total cholesterol levels * PNPLA3 CG or GG genotype' to evaluate whether the regression coefficients for these parameters differed significantly between carriers and non-carriers of the PNPLA3 G risk allele (heterozygotes and homozygotes combined). This analysis showed that the contribution of triglyceride levels to ALT levels was significantly higher in $G$ allele carriers compared to non-carriers ( $p=0.045)$, with a regression coefficient of $\beta=0.128$ compared to $\beta=0.040$ (supplemental table 1 ). Separate regression analysis for all three PNPLA3 genotypes was not added to the main article, due to limited sample size of children with GG genotype, but can be found in (supplemental table 2).

Stratified analysis for TM6SF2 genotype identified $\operatorname{sex}(\beta=0.127, p<0.001)$, total cholesterol levels $(\beta=0.053, p=0.022)$, triglycerides levels $(\beta=0.055, p=0.036)$ and HOMA-IR $(\beta=0.023$, $\mathrm{p}=0.023$ ) as predictors of logALT in non-carriers (table 5 ). In a similar regression model, no significant predictors of (log)ALT levels were identified in carriers of the TM6SF2 T risk allele ( $p$-value of the F-test of the overall significance of the regression model was $>0.05$ ). Because of the relatively small sample size of children carrying the TM6SF2 risk allele, a limited regression analysis was performed (without the possible confounders BMI z-score and age), which was also not a significant model. 


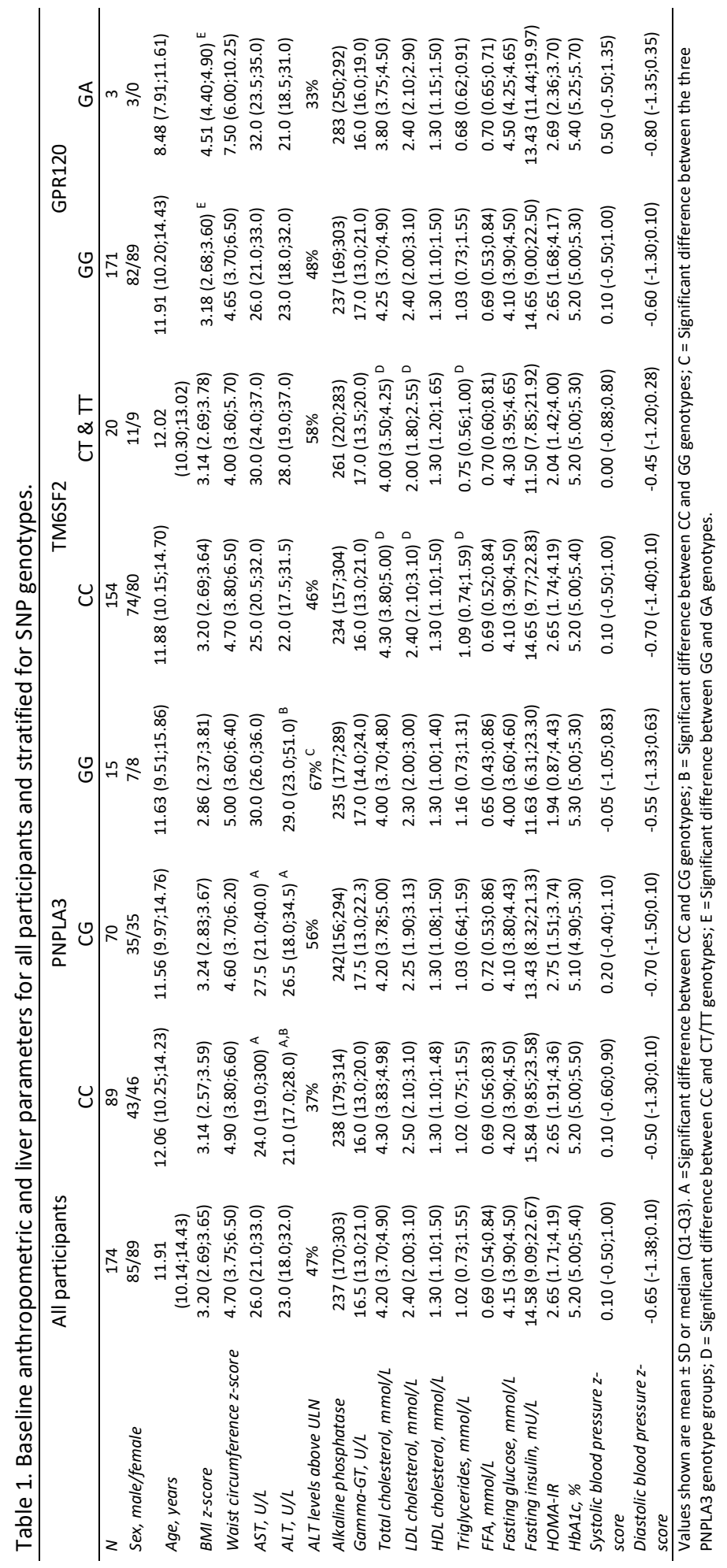


Table 2. Correlation coefficients for cardiometabolic parameters and ALT levels, for all participants and stratified for SNP genotypes

\begin{tabular}{|c|c|c|c|c|c|c|c|}
\hline & \multirow{2}{*}{\multicolumn{2}{|c|}{$\begin{array}{l}\text { All } \\
\text { participants } \\
\mathrm{N}\end{array}$}} & \multicolumn{3}{|c|}{ PNPLA3 } & \multicolumn{2}{|c|}{ TM6SF2 } \\
\hline & & & $\mathrm{CC}$ & CG & GG & $\mathrm{CC}$ & $\mathrm{CT} \& \mathrm{TT}$ \\
\hline$N$ & & 171 & 86 & 70 & 15 & 152 & 19 \\
\hline BMI z-score & 171 & 0.107 & 0.04 & 0.135 & 0.255 & 0.102 & 0.169 \\
\hline Total cholesterol, $\mathrm{mmol} / \mathrm{L}$ & 171 & $0.183^{A}$ & $0.232^{A}$ & 0.191 & 0.429 & $0.206^{A}$ & 0.235 \\
\hline LDL cholesterol, $\mathrm{mmol} / \mathrm{L}$ & 171 & 0.136 & 0.139 & 0.187 & 0.177 & 0.149 & 0.232 \\
\hline HDL cholesterol, $\mathrm{mmol} / \mathrm{L}$ & 171 & -0.062 & 0.128 & -0.208 & -0.118 & -0.069 & -0.110 \\
\hline Triglycerides, $\mathrm{mmol} / \mathrm{L}$ & 171 & $0.213^{A}$ & 0.06 & $0.317^{\mathrm{A}}$ & $0.750^{\mathrm{A}}$ & $0.250^{A}$ & 0.132 \\
\hline$F F A, m m o l / L$ & 166 & -0.023 & 0.046 & -0.073 & -0.002 & 0.004 & -0.297 \\
\hline Fasting glucose, $\mathrm{mmol} / \mathrm{L}$ & 169 & 0.045 & 0.044 & 0.085 & -0.023 & 0.037 & 0.01 \\
\hline Fasting insulin, $m U / L$ & 148 & $0.164^{\mathrm{A}}$ & 0.011 & $0.275^{A}$ & $0.674^{A}$ & 0.147 & 0.378 \\
\hline HOMA-IR & 146 & 0.153 & 0.003 & $0.283^{A}$ & $0.730^{A}$ & 0.130 & 0.376 \\
\hline$H b A 1 c, \%$ & 165 & 0.091 & 0.127 & 0.213 & -0.192 & 0.055 & 0.384 \\
\hline Systolic blood pressure z-score & 166 & -0.021 & 0.038 & -0.073 & -0.034 & -0.012 & 0.008 \\
\hline Diastolic blood pressure z-score & 166 & 0.054 & 0.195 & -0.102 & 0.187 & 0.065 & -0.002 \\
\hline
\end{tabular}

Table 3. Determinants of logALT levels in the whole group of participants

\begin{tabular}{lcc}
\hline & $B$ & P-value \\
\hline & $\mathrm{R}^{2}=0.354$ & \\
Sex, male & $\mathrm{F}=7.400(\mathrm{p}<0.001)$ & $<0.001$ \\
Age, years & 0.133 & 0.585 \\
BMI z-score & -0.003 & 0.985 \\
Total cholesterol, mmol/L & 0.000 & 0.004 \\
Triglycerides, mmol/L & 0.061 & 0.008 \\
HOMA-IR & 0.064 & 0.001 \\
PNPLA CG & 0.025 & 0.002 \\
PNPLA GG & 0.101 & $<0.001$ \\
TM6SF2 CT\&TT & 0.203 & 0.008 \\
GPR120 & 0.124 & 0.610 \\
\hline B'SSTHZ & -0.058 & \\
\hline
\end{tabular}

B's shown are unstandardized.

Table 4. Determinants of logALT levels, stratified for PNPLA3 genotypes

\begin{tabular}{|c|c|c|c|c|}
\hline & \multicolumn{2}{|c|}{ PNPLA3 CC } & \multicolumn{2}{|c|}{ PNPLA3 CG \& GG } \\
\hline & B & P-value & B & P-value \\
\hline & $R^{2}=0.204$ & & $R^{2}=0.406$ & \\
\hline & $F=2.858(p=0.015)$ & & $F=7.399(p<0.001)$ & \\
\hline Sex, male & 0.140 & 0.005 & 0.127 & 0.003 \\
\hline Age, years & -0.012 & 0.158 & 0.001 & 0.879 \\
\hline BMI z-score & -0.021 & 0.488 & 0.012 & 0.717 \\
\hline Total cholesterol, $\mathrm{mmol} / \mathrm{L}$ & 0.044 & 0.183 & 0.049 & 0.087 \\
\hline Triglycerides, $\mathrm{mmol} / \mathrm{L}$ & 0.035 & 0.262 & 0.108 & 0.009 \\
\hline$H O M A-I R$ & 0.025 & 0.011 & 0.027 & 0.018 \\
\hline
\end{tabular}

B's shown are unstandardized. 
Table 5. Determinants for logALT levels for TM6SF2 CC genotype

\begin{tabular}{lcc}
\hline & \multicolumn{2}{c}{ TM6SF2 CC } \\
\cline { 2 - 3 } & $\mathrm{R}^{2}=0.231$ & P-value \\
\hline Sex, male & $\mathrm{F}=6.016(\mathrm{p}<0.001)$ & \\
Age, years & 0.127 & $<0.001$ \\
BMl z-score & -0.004 & 0.537 \\
Total cholesterol, $\mathrm{mmol} / \mathrm{L}$ & -0.007 & 0.787 \\
Triglycerides, $\mathrm{mmol} / \mathrm{L}$ & 0.053 & 0.022 \\
HOMA-IR & 0.055 & 0.036 \\
\hline
\end{tabular}

B's shown are unstandardized.

\section{Health parameters in the different genotypes}

ALT levels were significantly higher in PNPLA3 CG and GG allele carriers compared to CC genotype (table 1). ALT and AST levels were also slightly increased among carriers of the TM6SF2 minor allele compared to non-carriers, however this difference did not reach significance. Interestingly, children with one or two PNPLA3 minor risk alleles did not show a deteriorated metabolic profile (table 1). Even though triglyceride and HOMA-IR levels were identified as a significant predictor of ALT levels in children carrying a PNPLA3 G allele and these children had higher ALT levels, the levels of triglyceride or HOMA-IR were not higher as compared to children without a PNPLA3 risk allele (table $1 \&$ figure 1 ). Also, while the percentage of children with ALT levels above the upper limit of normal was $58 \%$ in carriers of the PNPLA3 G allele compared to $37 \%$ in non-carriers $(p=0.009)$, the percentage of children with triglyceride levels above the upper limit of normal was $26 \%$ in both groups $(p=1.000)$.

Carriers of at least one TM6SF2 T risk allele showed a more beneficial cardiovascular risk profile, with significantly lower serum total cholesterol, LDL cholesterol and triglyceride concentrations. There was no significant difference in age, sex or BMI z-score between the different PNPLA3 or TM6SF2 genotypes (table 1). 

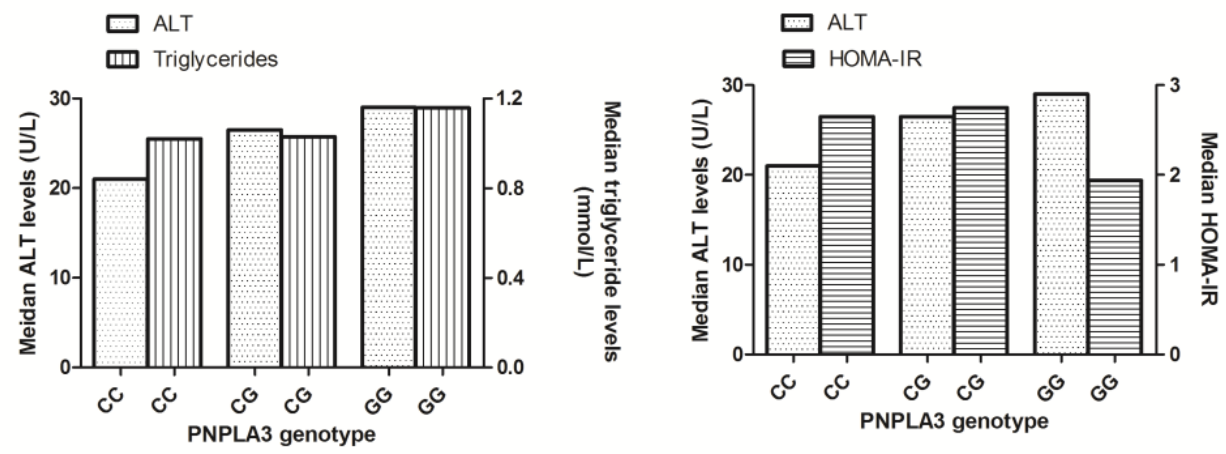

Figure 1. Median ALT levels per PNPLA3 genotype, compared to median triglyceride and HOMA-IR levels.

\section{Discussion}

In this study, we demonstrated that determinants of ALT levels, as a marker for NAFLD, are not the same in all children, but that they are SNP and genotype-specific. Further, high ALT levels can be found in children with overweight or obesity and a genetic susceptibility to NAFLD even when additional (metabolic) risk factors specific for that genetic variant are within the normal range. This suggests that carriers of risk SNPs such as the PNPLA3 G allele are sensitive to TG concentrations already at a lower range.

Our results showed that carrying the PNPLA3 risk allele is associated with increased ALT and AST levels, reflecting liver injury (28) and suggestive of steatohepatitis, in children with overweight, obesity and morbid obesity. These results are fully in line with findings of previous studies, which also found elevated ALT levels in children carrying the PNPLA3 risk variant $(29,30)$. In our study, the TM6SF2 T allele showed a less pronounced association with liver injury, i.e. only mildly elevated AST and ALT concentrations in carriers of the E167K variant. Previously, Eslam et al. found an association between the TM6SF2 T allele and the risk of developing liver steatosis and fibrosis, but not with ALT, AST levels or histological inflammation in a group of adults with NAFLD (31). However, other studies did find an association between the TM6SF2 polymorphism and increased ALT levels in (obese) children (32) and adults (33).

Regression analysis showed that determinants of ALT levels were not the same in the different subgroups, but differed depending on genetic variants. We found that in the whole group of children, male sex, total cholesterol levels, triglyceride levels and HOMA-IR were important determinants of ALT levels, in addition to PNPLA3 and TM6SF2 genotype. Previously, Purcell et al. also identified male sex, overweight/obesity, presence of the 
metabolic syndrome and a high HOMA-IR as risk factors for elevated ALT levels in a large population of Mexican children (34). However, genetic predisposition and separate metabolic parameters, rather than the presence or absence of the metabolic syndrome, were not taken into account in that study.

To the best of our knowledge, genotype-specific determinants of ALT levels in children were not studied previously. Interestingly, stratified analyses showed that the determinants of ALT levels were specific for the different genetic variants. In addition to sex and HOMA-IR, triglyceride levels were identified as a significant determinant of ALT levels in PNPLA3 G risk allele carriers, but not in non-carriers. Sex, total cholesterol levels, triglyceride levels and HOMA-IR were significant determinants of logALT in TM6SF2 noncarriers, but not in carriers of the TM6SF2 risk allele. These results show that it is important to consider that determinants of ALT levels cannot be generalized across populations, but that they differ between different subgroups, for instance based on genetic predisposition. This difference between genotypes might also affect therapeutic strategies and should therefore be taken into account in studies towards novel treatment options.

Interestingly, even though triglyceride and HOMA-IR levels were identified as significant predictors of ALT levels in children carrying a PNPLA3 G allele, these children did not have elevated triglyceride levels or HOMA-IR, compared to children without a PNPLA3 risk allele.

As NAFLD is often assumed to be the hepatic component of the metabolic syndrome (35), we were therefore surprised to find that carriers of the PNPLA3 G risk allele did not show a more disturbed cardiometabolic profile compared to CC homozygotes despite higher ALT levels. Previous studies have provided conflicting results on the association between PNPLA3 genotypes and metabolic abnormalities. Goran et al. showed no association between PNPLA3 genotype and lipid and glucose metabolism, except for lower HDL in children with the GG genotype (13), whereas Mangge et al. did find an association between the PNPLA3 G allele and the presence of the metabolic syndrome across all ages (29).

It is interesting to consider why, despite this strong association between triglyceride levels and HOMA-IR and ALT levels in G allele carriers, we did find increased ALT levels in these children, but not increased triglyceride or HOMA-IR levels. These results could suggest different scenarios, for instance that there is a difference in sensitivity for the possible harmful effects in the liver of PNPLA3 G allele carriers, compared to non-carriers. Although studies towards further elucidating the exact underlying mechanism by which the PNPLA3 risk variant promotes the development of NAFLD are still ongoing, the current idea is that 
the risk variant leads to TG accumulation in the liver because the PNPLA3-148M protein accumulates on lipid droplets, thereby impairing TG mobilization from these droplets (36). Additionally, the fact that we did find increased ALT levels, without increased triglyceride or HOMA-IR levels, suggests that besides the parameters in our models, other factors must play an important role in explaining the variation in the increase of ALT levels in risk allele carriers.

Further studies are needed to gain more insight in the underlying mechanisms of the development of NAFLD in genetically predisposed patients. Our results show that determinants of liver enzyme levels are genotype-specific and that, in risk allele carriers, increased ALT levels can be present with metabolic parameters within the normal range. These results demonstrate that we cannot simply generalize factors involved in the development of NAFLD and suggest that underlying mechanisms might differ between children with and without a genetic predisposition. Since the genetic risk variants are quite common, it is important to take these findings into consideration both from a scientific as a clinical point of view.

\section{Conclusion}

Determinants of ALT levels cannot be generalized across all children, but are SNP and genotype-specific. Furthermore, this study shows that high ALT levels can be found in children with overweight and a genetic predisposition, even when genotype-specific determinants of ALT levels such as TG levels in PNPLA3 risk allele carriers, are within the normal range. 


\section{References}

1. Cohen JC, Horton JD, Hobbs HH. Human fatty liver disease: old questions and new insights. Science. 2011;332(6037):1519-1523.

2. White DL, Kanwal F, El-Serag HB. Association between nonalcoholic fatty liver disease and risk for hepatocellular cancer, based on systematic review. Clinical gastroenterology and hepatology : the official clinical practice journal of the American Gastroenterological Association. 2012;10(12):1342-1359.e1342.

3. Valenti L, Bugianesi E, Pajvani U, Targher G. Nonalcoholic fatty liver disease: cause or consequence of type 2 diabetes? Liver international : official journal of the International Association for the Study of the Liver. 2016;36(11):1563-1579.

4. Sookoian S, Pirola CJ. Non-alcoholic fatty liver disease is strongly associated with carotid atherosclerosis: a systematic review. Journal of hepatology. 2008;49(4):600-607.

5. Suomela $\mathrm{E}$, Oikonen $\mathrm{M}$, Pitkanen $\mathrm{N}$, et al. Childhood predictors of adult fatty liver. The Cardiovascular Risk in Young Finns Study. Journal of hepatology. 2016;65(4):784-790.

6. Rich NE, Oji S, Mufti AR, et al. Racial and Ethnic Disparities in Nonalcoholic Fatty Liver Disease Prevalence, Severity, and Outcomes in the United States: A Systematic Review and Meta-analysis. Clinical gastroenterology and hepatology : the official clinical practice journal of the American Gastroenterological Association. 2018;16(2):198-210.e192.

7. Prokopowicz Z, Malecka-Tendera E, Matusik P. Predictive Value of Adiposity Level, Metabolic Syndrome, and Insulin Resistance for the Risk of Nonalcoholic Fatty Liver Disease Diagnosis in Obese Children. Canadian journal of gastroenterology \& hepatology. 2018;2018:9465784.

8. Papandreou D, Karavetian M, Karabouta Z, Andreou E. Obese Children with Metabolic Syndrome Have 3 Times Higher Risk to Have Nonalcoholic Fatty Liver Disease Compared with Those without Metabolic Syndrome. International journal of endocrinology. 2017;2017:2671692.

9. Speliotes EK, Yerges-Armstrong LM, Wu J, et al. Genome-wide association analysis identifies variants associated with nonalcoholic fatty liver disease that have distinct effects on metabolic traits. PLoS Genet. 2011;7(3):e1001324.

10. Romeo S, Kozlitina J, Xing C, et al. Genetic variation in PNPLA3 confers susceptibility to nonalcoholic fatty liver disease. Nature genetics. 2008;40(12):1461-1465.

11. Sookoian S, Pirola CJ. Meta-analysis of the influence of $1148 \mathrm{M}$ variant of patatin-like phospholipase domain containing 3 gene (PNPLA3) on the susceptibility and histological severity of nonalcoholic fatty liver disease. Hepatology (Baltimore, Md). 2011;53(6):18831894.

12. Rotman Y, Koh C, Zmuda JM, Kleiner DE, Liang TJ. The association of genetic variability in patatin-like phospholipase domain-containing protein 3 (PNPLA3) with histological severity of nonalcoholic fatty liver disease. Hepatology (Baltimore, Md). 2010;52(3):894-903.

13. Goran MI, Walker R, Le KA, et al. Effects of PNPLA3 on liver fat and metabolic profile in Hispanic children and adolescents. Diabetes. 2010;59(12):3127-3130.

14. Huang $\mathrm{Y}, \mathrm{He} \mathrm{S}$, Li JZ, et al. A feed-forward loop amplifies nutritional regulation of PNPLA3. Proc Natl Acad Sci U S A. 2010;107(17):7892-7897. 
15. Krawczyk M, Portincasa P, Lammert F. PNPLA3-associated steatohepatitis: toward a genebased classification of fatty liver disease. Seminars in liver disease. 2013;33(4):369-379.

16. Smagris E, BasuRay S, Li J, et al. Pnpla3I148M knockin mice accumulate PNPLA3 on lipid droplets and develop hepatic steatosis. Hepatology (Baltimore, Md). 2015;61(1):108-118.

17. Chen LZ, Xia HHX, Xin YN, Lin ZH, Xuan SY. TM6SF2 E167K Variant, a Novel Genetic Susceptibility Variant, Contributing to Nonalcoholic Fatty Liver Disease. Journal of Clinical and Translational Hepatology. 2015;3(4):265-270.

18. Pirola CJ, Sookoian S. The dual and opposite role of the TM6SF2-rs58542926 variant in protecting against cardiovascular disease and conferring risk for nonalcoholic fatty liver: A meta-analysis. Hepatology (Baltimore, Md). 2015;62(6):1742-1756.

19. Goffredo M, Caprio S, Feldstein AE, et al. Role of TM6SF2 rs58542926 in the pathogenesis of nonalcoholic pediatric fatty liver disease: A multiethnic study. Hepatology (Baltimore, Md). 2016;63(1):117-125.

20. Oh DY, Talukdar S, Bae EJ, et al. GPR120 is an omega-3 fatty acid receptor mediating potent anti-inflammatory and insulin-sensitizing effects. Cell. 2010;142(5):687-698.

21. Marzuillo $P$, Grandone $A$, Conte $M$, et al. Novel association between a nonsynonymous variant $(\mathrm{R} 27 \mathrm{OH})$ of the G-protein-coupled receptor 120 and liver injury in children and adolescents with obesity. Journal of pediatric gastroenterology and nutrition. 2014;59(4):472-475.

22. Rijks JM, Plat J, Mensink RP, Dorenbos E, Buurman WA, Vreugdenhil AC. Children With Morbid Obesity Benefit Equally as Children With Overweight and Obesity From an Ongoing Care Program. J Clin Endocrinol Metab. 2015;100(9):3572-3580.

23. Cole TJ, Lobstein T. Extended international (IOTF) body mass index cut-offs for thinness, overweight and obesity. Pediatr Obes. 2012;7(4):284-294.

24. Fredriks AM, van Buuren S, Fekkes M, Verloove-Vanhorick SP, Wit JM. Are age references for waist circumference, hip circumference and waist-hip ratio in Dutch children useful in clinical practice? Eur J Pediatr. 2005;164(4):216-222.

25. Schwimmer JB, Dunn W, Norman GJ, et al. SAFETY study: alanine aminotransferase cutoff values are set too high for reliable detection of pediatric chronic liver disease. Gastroenterology. 2010;138(4):1357-1364, 1364 e1351-1352.

26. Friedewald WT, Levy RI, Fredrickson DS. Estimation of the concentration of low-density lipoprotein cholesterol in plasma, without use of the preparative ultracentrifuge. Clin Chem. 1972;18(6):499-502.

27. Wuhl E, Witte K, Soergel M, Mehls O, Schaefer F. Distribution of 24-h ambulatory blood pressure in children: normalized reference values and role of body dimensions. J Hypertens. 2002;20(10):1995-2007.

28. Senior JR. Alanine aminotransferase: a clinical and regulatory tool for detecting liver injurypast, present, and future. Clinical pharmacology and therapeutics. 2012;92(3):332-339.

29. Mangge H, Baumgartner BG, Zelzer S, et al. Patatin-like phospholipase 3 (rs738409) gene polymorphism is associated with increased liver enzymes in obese adolescents and metabolic syndrome in all ages. Aliment Pharmacol Ther. 2015;42(1):99-105. 
30. Krawczyk M, Liebe R, Maier IB, Engstler AJ, Lammert F, Bergheim I. The Frequent Adiponutrin (PNPLA3) Variant p.lle148Met Is Associated with Early Liver Injury: Analysis of a German Pediatric Cohort. Gastroenterol Res Pract. 2015;2015:205079.

31. Eslam M, Mangia A, Berg T, et al. Diverse impacts of the rs58542926 E167K variant in TM6SF2 on viral and metabolic liver disease phenotypes. Hepatology (Baltimore, Md). 2016.

32. Grandone A, Cozzolino D, Marzuillo P, et al. TM6SF2 Glu167lys polymorphism is associated with low levels of LDL-cholesterol and increased liver injury in obese children. Pediatric obesity. 2016;11(2):115-119.

33. Dongiovanni P, Petta S, Maglio $C$, et al. Transmembrane 6 superfamily member 2 gene variant disentangles nonalcoholic steatohepatitis from cardiovascular disease. Hepatology (Baltimore, Md). 2015;61(2):506-514.

34. Purcell M, Flores YN, Zhang ZF, Denova-Gutierrez E, Salmeron J. Prevalence and predictors of alanine aminotransferase elevation among normal weight, overweight and obese youth in Mexico. Journal of digestive diseases. 2013;14(9):491-499.

35. Tarantino $\mathrm{G}$, Finelli $\mathrm{C}$. What about non-alcoholic fatty liver disease as a new criterion to define metabolic syndrome? World J Gastroenterol. 2013;19(22):3375-3384.

36. BasuRay S, Smagris E, Cohen JC, Hobbs HH. The PNPLA3 variant associated with fatty liver disease (I148M) accumulates on lipid droplets by evading ubiquitylation. Hepatology (Baltimore, Md). 2017;66(4):1111-1124. 
Supplemental table 1. Determinants of logALT levels in the whole group of participants including interaction term for PNPLA3 genotype and triglyceride levels

\begin{tabular}{lcc}
\hline & $B$ & P-value \\
\hline & $\mathrm{R}^{2}=0.356$ & \\
Sex, male & $\mathrm{F}=8.341(\mathrm{p}<0.001)$ & $<0.001$ \\
Age, years & 0.128 & 0.459 \\
BMI z-score & -0.004 & 0.781 \\
Total cholesterol, mmol/L & -0.006 & 0.013 \\
Triglycerides, $m$ mol/L & 0.052 & 0.137 \\
HOMA-IR & 0.040 & 0.001 \\
PNPLA CG $+G G$ & 0.024 & 0.802 \\
PNPLA CG $+G G{ }^{*}$ triglycerides & 0.015 & 0.045 \\
TM6SF2 CT\&TT & 0.088 & 0.009 \\
\hline B'S Shown are unstandardized. & 0.120 &
\end{tabular}

Supplemental table 2. Determinants for logALT levels, stratified for all three PNPLA3 genotypes

\begin{tabular}{|c|c|c|c|c|c|c|}
\hline & \multicolumn{2}{|c|}{ PNPLA3 CC genotype } & \multicolumn{2}{|c|}{ PNPLA3 CG genotype } & \multicolumn{2}{|c|}{ PNPLA3 GG genotype } \\
\hline & B & P-value & B & $\mathrm{P}$-value & B & P-value \\
\hline & \multicolumn{2}{|c|}{$R^{2}=0.204$} & \multicolumn{2}{|c|}{$R^{2}=0.402$} & \multicolumn{2}{|c|}{$R^{2}=0.884$} \\
\hline & \multicolumn{2}{|c|}{$F=2.858 \quad(p=0.015)$} & \multicolumn{2}{|c|}{$F=5.831(p<0.001)$} & \multicolumn{2}{|c|}{$F=7.647(p=0.013)$} \\
\hline Sex, male & 0.140 & 0.005 & 0.157 & 0.002 & 0.155 & 0.069 \\
\hline Age, years & -0.012 & 0.158 & 0.001 & 0.946 & 0.008 & 0.466 \\
\hline BMI z-score & -0.021 & 0.488 & -0.007 & 0.851 & 0.049 & 0.235 \\
\hline Total cholesterol, $\mathrm{mmol} / \mathrm{L}$ & 0.044 & 0.183 & 0.051 & 0.108 & 0.179 & 0.042 \\
\hline Triglycerides, $\mathrm{mmol} / \mathrm{L}$ & 0.035 & 0.262 & 0.082 & 0.082 & 0.198 & 0.012 \\
\hline$H O M A-I R$ & 0.025 & 0.011 & 0.033 & 0.020 & -0.002 & 0.883 \\
\hline
\end{tabular}

B's shown are unstandardized. 


\section{Chapter 8}

Low levels of antibodies against oxidation specific epitopes on oxLDL are associated with parameters reflecting NAFLD and metabolic health in children

Kylie Karnebeek Anita C.E. Vreugdenhil

Tim Hendrikx Jogchum Plat Yvonne Oligschlaeger Ronit Shiri-Sverdlov

Submitted 


\section{Abstract}

\section{Background}

Oxidized low-density lipoproteins (oxLDL) play a role in the development of non-alcoholic fatty liver disease (NAFLD). Previously, we demonstrated that increased circulatory IgM antibodies targeting oxidation specific epitopes (OSE) on oxLDL are protective against steatohepatitis in rodent models and adult NAFLD patients. This protective effect may be decreased in obesity, due to an impaired immune function. However, the role of oxLDL and IgM antibodies against OSE in the development of NAFLD in children remains unknown. Here, we aimed to evaluate levels of OSE-specific IgM antibodies in children of different weight classifications, to assess associations between these antibody levels and parameters reflecting NAFLD and to assess if these associations were liver-specific.

\section{Methods}

Two-hundred fifty-two children with overweight or (morbid) obesity and 31 lean children were included. IgM titers against copper-oxidized (Cu-ox)-LDL, malondialdehyde (MDA)LDL and the P1-mimitope and total IgM were measured, as well as liver enzyme and cytokeratin-18 levels, serum lipids, blood pressure, retinal microvasculature, cell-adhesion molecules, glucose, insulin, HOMA-IR, HbA1c, cytokines, chemokines and hsCRP.

\section{Results}

IgM levels against Cu-oxLDL were significantly lower in morbidly obese children, compared to lean children. Low titers of P1-IgM and Cu-oxLDL-IgM were associated with higher liver enzyme levels. Lower P1-IgM was a determinant for elevated logALT levels, and there was a trend for Cu-oxLDL-IgM and MDA-IgM. For logGGT levels low MDA-IgM levels were a significant determinant and there was a trend for P1-IgM. Low MDA-IgM and P1-IgM were determinants of higher fasting glucose levels, HBA1c and logHOMA-IR. Low MDA-IgM levels were associated with higher $\operatorname{logSAA}$ and $\log$ IL-6 levels, indicative of systemic inflammation.

\section{Conclusion}

Low titers of antibodies against OSE on oxLDL are associated with higher liver enzyme levels in obese children. These data suggest that low OSE-specific IgM antibodies might play a role in the etiology of disturbed liver function, however whether it is liver-specific remains to be elucidated since low OSE-specific antibodies were also related to systemic inflammation and an unfavorable glucose metabolism. 


\section{Introduction}

The current obesity epidemic has been associated with an increased risk of disease development, including diabetes type II, cardiovascular disease (CVD) and non-alcoholic fatty liver disease (NAFLD). Elevated serum low-density lipoprotein (LDL) concentrations as well as oxidative stress are characteristics observed in all three diseases (1-5). LDL particles can become oxidized under the influence of oxidative stress. These oxidized LDL (oxLDL) particles get internalized by scavenger cells of the innate immune system, like macrophages, leading to the formation of pro-inflammatory foam cells in the vascular wall. Subsequent inflammatory responses contribute to the process of atherosclerosis and cardiovascular disease (CVD) development $(6,7)$. Besides these well-recognized effects, previous studies in adults have also shown a role for oxLDL in the development of NAFLD (8-10). Similarly, it has been shown that children with obesity (11) and a higher cardiometabolic risk (12) have higher circulating levels of oxLDL. However, the associations between oxLDL and NAFLD development in children are not yet known.

During the oxidation of LDL particles, oxidation-specific epitopes (OSEs) are formed on the surface of the oxLDL particles. These OSEs are important targets of natural immunoglobulin M (IgM) antibodies, that can bind to newly formed OSEs on oxLDL. The antibodies neutralize the pathological effects of OSEs by blocking the subsequent formation of foam cells (7), thereby preventing an inflammatory response (13). Indeed, using hyperlipidemic mice, we and others have shown that OSE-specific IgM antibodies are protective against the development of non-alcoholic steatohepatitis (NASH)(10). Moreover, we have previously shown in adults that OSE-specific IgM levels were lower in patients with NAFLD compared to healthy controls (14). Since obesity in adults has been associated with a general impaired immune responsiveness (15), the formation and consequent protective effect of circulating OSE-specific IgM antibodies might be decreased in people with obesity. Therefore, a remaining question is whether the observed lower OSE-specific IgM levels in the NAFLD population were independent from their apparent obesity or not.

In contrast to the aforementioned associations between OSE-specific antibodies and NAFLD in adults, the associations between antibodies against oxLDL and NAFLD in children are not yet known. Therefore, the aim of this study was to assess whether OSE-specific IgM antibodies levels are also associated with elevated liver parameters in children with different levels of overweight and (morbid) obesity. Additionally, since the development of NAFLD has been associated with elaborate cardiometabolic and inflammatory aberrations, we aimed to evaluate whether associations between OSE-specific antibodies were liver-specific or may be related to the full metabolic imbalance spectrum. 


\section{Methods}

\section{Participants}

In this study, 252 children with overweight, obesity and morbid obesity from the Centre for Overweight Adolescent and Children's Healthcare (COACH) at the Maastricht University Medical Centre were included. Children and adolescents with overweight, obesity and morbid obesity are evaluated, monitored and guided in this childhood obesity expertise centre as described previously (16). All data described in this study were collected prior to participation in a lifestyle intervention.

Additionally, 31 lean children that visited the pediatric outpatient clinic in the Maastricht University Medical Centre with functional abdominal pain and without signs of underlying disease, were included. Of these children, age, sex and BMI were known and blood samples were available for measuring IgM antibody levels. The study was conducted according to the guidelines of the Declaration of Helsinki and approved by the medical ethical committee of the MUMC+.

\section{IgM antibody measurements}

Chemiluminescent ELISA was performed as described in more detail previously $(14,17)$. In short, anti-human IgM, MDA-LDL antigens and Cu-oxLDL antigens were added to a white 96-well microtitration plate in $5 \mu \mathrm{g} / \mathrm{ml}$ concentrations in $50 \mu \mathrm{g} / \mathrm{ml}$ phosphate-buffered saline-EDTA (Microfluorll roundbottom; Thermo, Rochester, New York, USA). P1 peptide (Peptide 2.0 Inc.) was coated in $10 \mu \mathrm{g} / \mathrm{ml}$ concentrations in $\mathrm{NaHCO}_{3}$. All plates were incubated overnight at $4^{\circ} \mathrm{C}$. After washing and blocking, the plates were incubated with the plasma samples in $1 \%$ bovine serum albumin in Tris-buffered saline with EDTA. Alkaline-phophatase-labeled goat anti-human IgM ( $\mu$-chain specific; Sigma-Aldrich, Vienna, Austria) was used for detection.

\section{Anthropometric measurements}

Weight was measured on a digital scale (Seca) and height was measured using a digital stadiometer (De Grood Metaaltechniek), while children were barefoot and in underwear. Body Mass Index (BMI) was calculated and BMI z-scores were obtained using a growth analyser (Growth Analyser VE). Children were classified as overweight, obese or morbidly obese based on International Obesity Task Force criteria (18). 


\section{Parameters reflecting liver health}

Serum alanine transaminase (ALT), aspartate transaminase (AST) and gamma-glutamyl transferase (GGT) were measured with the Cobas 8000 modular analyser (Roche). For analyses in this study, the upper limit of normal (ULN) used for ALT was $22.1 \mathrm{U} / \mathrm{L}$ for girls and $25.8 \mathrm{U} / \mathrm{L}$ for boys (19). Cytokeratin 18 was measured with the M30-apoptosense ELISA kit (PEVIVA), in a subgroup of 84 children.

\section{Parameters reflecting systemic inflammation}

Plasma concentrations of systemic inflammatory markers (Interleukin (IL)-6, IL-8 and tumor necrosis factor (TNF)- $\alpha$ ), as well as the acute phase proteins C-reactive protein (CRP) and serum amyloid A (SAA) were measured with a commercially available Multi Spot ELISA kit (Meso Scale Discovery, Rockville, USA).

\section{Parameters reflecting glucose metabolism}

Fasting blood glucose was measured with the Cobas 8000 modular analyser and fasting serum insulin levels were measured with the Immulite 1000 (Siemens). Glycosylated hemoglobin (HbA1c) was measured with the HPLC Variant II (Bio-Rad Laboratories). Insulin resistance was evaluated by calculating the homeostasis model assessment for insulin resistance (HOMA-IR) (HOMA-IR = fasting glucose $(\mathrm{mmol} / \mathrm{L}) \times$ fasting insulin $(\mu \mathrm{U} / \mathrm{L}) / 22.5)$.

\section{Parameters reflecting cardiovascular health}

Fasting serum total cholesterol, high-density lipoprotein (HDL)-cholesterol, triglycerides and free fatty acid (FFA) levels were measured with the Cobas 8000 modular analyser. LDL cholesterol was calculated using the Friedewald equation (20). Blood pressure was measured approximately 20 times using the Mobil-O-Graph (I.E.M. GmbH) and blood pressure z-scores were calculated according to reference values for height and sex, based on the average of all measurements (21). To assess arterial stiffness, the augmentation index (Alx) was measured (Mobil-O-Graph). Alx values were adjusted to a standardized heart rate of 75 beats per minute. Retinal vascular images of the right eye were made (TRC-NW300; Topcon Co) to assess microvascular health. The central retinal arteriolar equivalent (CRAE), the central retinal venular equivalent (CRVE), as well as their ratio were calculated as described previously (22). Circulating concentrations of shed cellular adhesion molecules (soluble (s) E-selectin, sICAM-1, and SVCAM-1) were measured with a 
commercially available Multi Spot ELISA kit (Meso Scale Discovery, Rockville, USA) as a measure of endothelial function.

\section{Statistical analysis}

Statistical analyses were performed with IBM SPSS statistics 22.0. Data was tested for normality with the Shapiro-Wilk test and were accordingly reported as mean \pm standard deviation or median (interquartile range). Statistical differences between groups were analyzed with an independent samples $t$-test, Mann-Whitney $U$ test, ANOVA or KruskalWallis test, depending on the distribution. Pearson or Spearman's correlation analyses were performed, depending on the distribution. Linear regression was performed to assess the contribution of the different IgM antibodies to parameters associated with liver and cardiovascular health, systemic inflammation and glucose metabolism. Regression models were corrected for age and/or BMI z-score, if there was a significant correlation between these variables and either the IgM antibody or dependent variable in the model. The models were corrected for sex if IgM antibody levels or dependent variable was significantly different between boys and girls. In the regression analysis, some parameters were log-transformed to achieve a normal distribution of the residuals of the models. Only significant outcomes and trends of the regression models are shown in the tables. For all analyses, a p-value $<0.05$ was considered statistically significant. For the ANOVA and Kruskal-Wallis tests, P-values were corrected for multiple testing.

\section{Results}

\section{Characteristics of the study population}

Two-hundred fifty-two children (104 boys, 148 girls; mean age $11.55 \pm 3.16$ years) with overweight $(n=42)$, obesity $(n=122)$ or morbid obesity $(n=88)$ and 31 lean children (19 boys, 12 girls; mean age $9.7 \pm 5.4$ ) were included in this study (table 1 ).

IgM titers against $\mathrm{Cu}$-oxLDL were significantly lower $(\mathrm{p}=0.037)$ in children with morbid obesity (35667 $\pm 15487 \mathrm{RLU} / 100 \mathrm{~ms}$ ) compared to the lean children (44998 \pm 20427 $\mathrm{RLU} / 100 \mathrm{~ms})$ and there was a trend ( $p=0.06)$ towards lower anti-Cu-oxLDL IgM levels in children with obesity (36550 $\pm 14262 \mathrm{RLU} / 100 \mathrm{~ms}$ ) compared to lean children (44998 \pm 20427 RLU/100ms). The concentrations of other OSE-specific IgM antibodies and total IgM did not differ between children with overweight or obesity and lean children. Only total IgM levels correlated inversely with BMI z-score ( $R=-0.164 ; p=0.027)$. 
Both the IgM titers against MDA-LDL and the IgM levels towards the specific P1 mimotope were significantly lower in boys compared to girls (MDA IgM: $45814 \pm 9976$ vs. $48592 \pm$ $10775 \mathrm{RLU} / 100 \mathrm{~ms}$ ( $p=0.041$ ); and P1-IgM $18043 \pm 10511$ vs. $21591 \pm 11595 \mathrm{RLU} / 100 \mathrm{~ms}$ $(p=0.014)$ in boys vs. girls respectively). Characteristics of the study population are presented in table 1.

\section{Lower titers of OSE-specific antibodies are associated with higher liver enzyme levels}

In order to evaluate possible associations between OSE-specific IgM antibodies and liver enzyme levels, correlation analyses were performed. Both Cu-oxLDL-IgM and P1-IgM levels were inversely correlated with ALT levels (respectively, $R=-0.152, p=0.019 ; R=$ -0.142, $p=0.028$ ) and GGT levels (respectively, $R=-0.152, p=0.019 ; R=-0.169, p=0.008$ ) in the total group of 252 children with overweight or obesity. AST levels were inversely correlated with total IgM levels $(R=-0.146, p=0.024)$, but not with the other OSE-specific antibodies. Stratification into subgroups based on ALT levels showed that CU-oxLDL-IgM and P1-IgM concentrations were significantly lower in children with ALT levels higher than two times the upper limit of normal (i.e. higher than $44 \mathrm{U} / \mathrm{L}$ in girls or $52 \mathrm{U} / \mathrm{L}$ in boys)( $\mathrm{n}=19)$, suggestive of liver inflammation, compared to children with normal ALT levels (i.e. values below the ULN; $n=136$ )(respectively, CuoxLDL-IgM: $27226 \pm 9755$ vs. $36925 \pm 14598$ RLU/100ms ( $p=0.006)$; and P1-IgM: $15297 \pm 8680$ vs. $20914 \pm 11267 \mathrm{RLU} / 100 \mathrm{~ms}$ ( $p=0.039$ ) respectively). The difference in MDA-IgM and total IgM levels between children with and without elevated ALT levels did not reach statistical significance (table 2). 


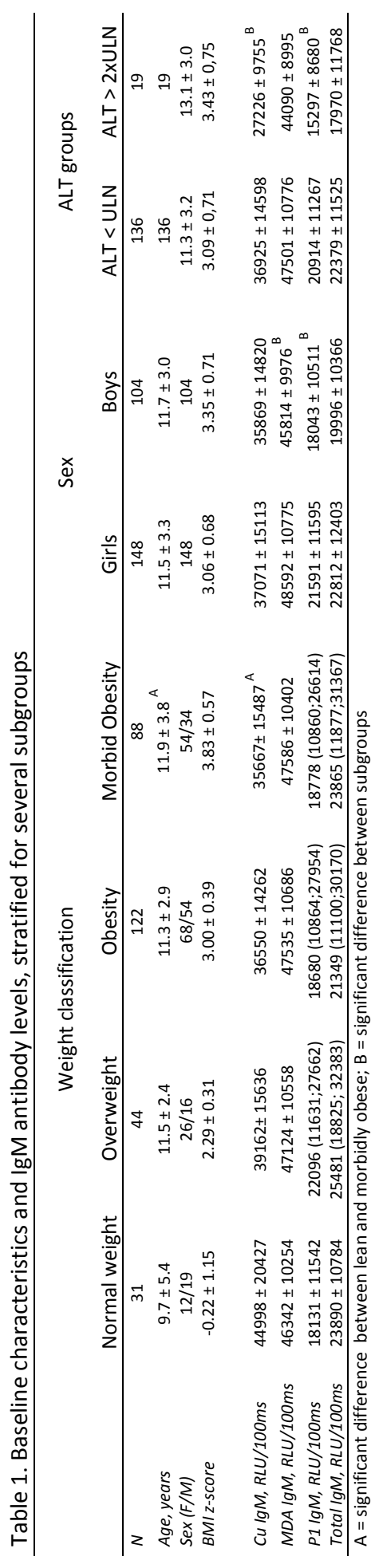




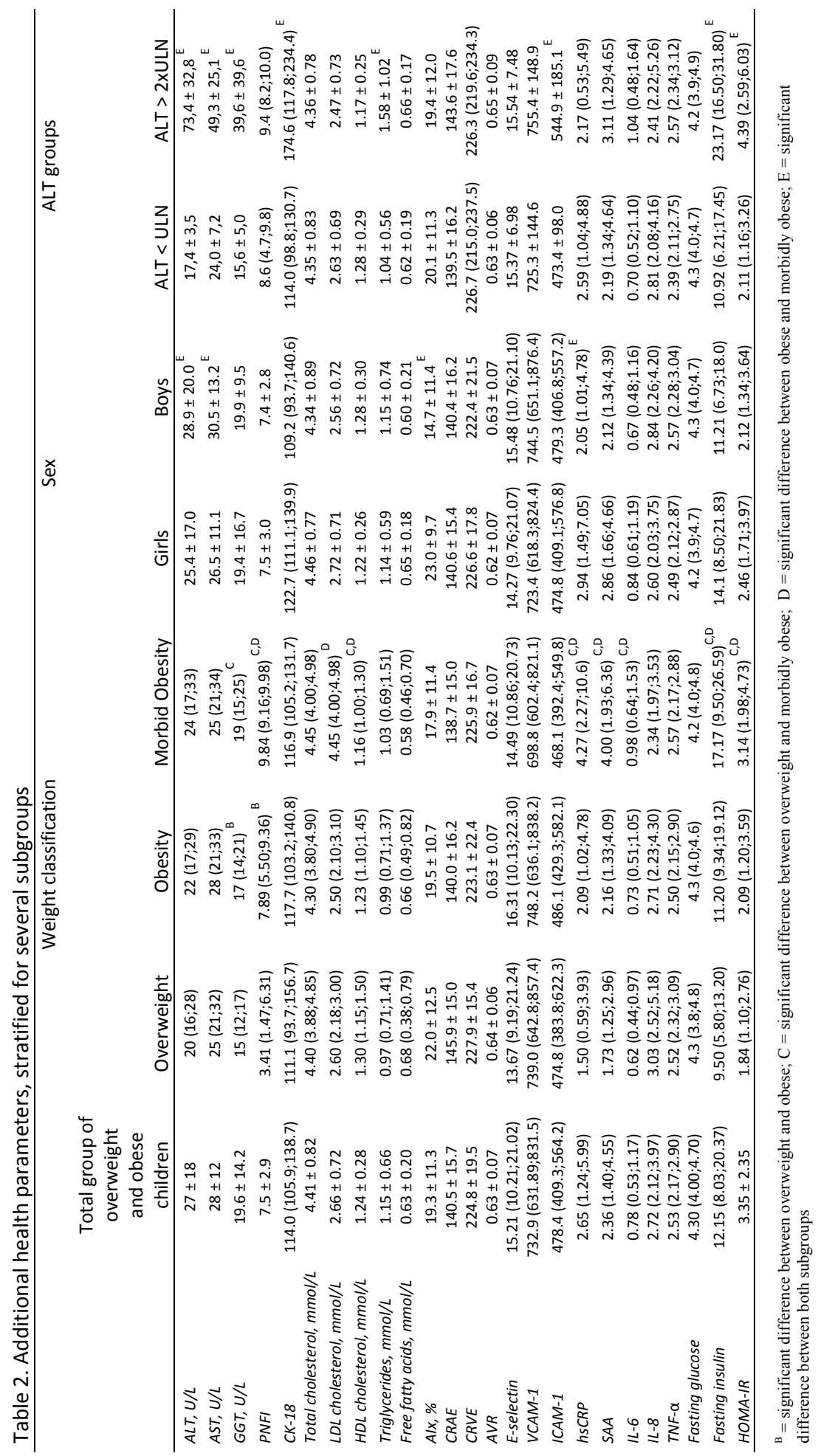


Next, regression analysis was performed to evaluate if the different OSE-specific IgM antibodies and total IgM were associated with liver parameters. P1-IgM levels were significantly associated with $\log A L T(\beta=-2.59 E-6 ; p=0.040)$. This was a negative association indicating that lower PI-IgM levels predicted higher logALT values. For both Cu-oxLDL-IgM levels $(\beta=-1.71 E-6 ; p=0.081)$ and MDA-IgM levels $(\beta=-2.34 E-6 ; p=0.076)$ there was a trend towards an inverse association with logALT levels (table 3). Additionally, MDA-IgM was identified as a significant inverse determinant of logGGT levels $(\beta=-2.83 E-6 ; p=0.017)$, and for P1-IgM levels there was a trend towards an inverse association $(\beta=-2.08 \mathrm{E}-6$; $\mathrm{p}=0.065$ ).

For $\log$ AST levels, only total IgM was identified as a significant inverse determinant $(\beta=-$ 1.92E-6; $p=0.030$ )(table 3). Altogether, these data suggest that lower concentrations of circulating antibodies against oxLDL are associated with higher liver enzymes and may potentially be involved in the early development of NAFLD in children.

Table 3. Associations between IgM antibody levels and liver parameters, identified with regression analysis

\begin{tabular}{|c|c|c|c|c|c|c|}
\hline & \multicolumn{2}{|c|}{$\log A L T$} & \multicolumn{2}{|c|}{$\log G G T$} & \multicolumn{2}{|c|}{$\log A S T$} \\
\hline & $\beta$ & $p$-value & $\beta$ & $p$-value & $\beta$ & $p$-value \\
\hline Cu IgM & $-1.71^{-6}$ & 0.081 & \multicolumn{2}{|c|}{ NS } & \multicolumn{2}{|c|}{ NS } \\
\hline P1 IgM & $-2.59^{-6}$ & 0.040 & $-2.08^{-6}$ & 0.065 & \multicolumn{2}{|c|}{ NS } \\
\hline MDA IgM & $-2.34^{-6}$ & 0.076 & $-2.83^{-6}$ & 0.017 & \multicolumn{2}{|c|}{ NS } \\
\hline Total IgM & $-1.86^{-6}$ & 0.105 & \multicolumn{2}{|c|}{ NS } & $-1.92^{-6}$ & 0.030 \\
\hline
\end{tabular}

\section{Anti-MDA IgM is a significant determinant of low-grade inflammation}

To determine whether the IgM antibodies were not only markers for liver health but also determinants of markers reflecting low-grade inflammation, regression analysis was performed. MDA-IgM was identified as a significant inverse determinant of logIL-6 ( $\beta=-$ 5.395E-6; $p=0.002)$ and $\operatorname{logSAA}(\beta=-6.352 E-6 ; p=0.023)$ (table 4). Also, there was a trend towards an association between $\mathrm{P} 1-\lg \mathrm{M}$ levels and $\log \mathrm{IL}-6(\beta=-3.130 \mathrm{E}-6 ; p=0.053)$. No associations between antibody titers and the other markers for inflammation were found. Altogether, these observations suggest that IgM antibodies against OSE-specific epitopes on oxLDL are also associated with systemic inflammation. Just as found for liver health, the direction illustrates that low antibody titers associate with increased low-grade inflammation. 


\section{MDA IgM, P1 IgM and total IgM are significant determinants of glucose metabolism}

Next, regression analysis was performed to determine whether OSE-specific antibodies were also associated with glucose, insulin and parameters reflecting insulin sensitivity. Here, MDA-IgM and P1-IgM levels were inversely associated with fasting glucose levels (respectively, $\beta=-1.22 \mathrm{E}-5 ; \mathrm{p}=0.005$ and $\beta=-1.095 \mathrm{E}-6 ; \mathrm{p}=0.009$ )(table 4). Moreover, $\mathrm{P} 1-\lg \mathrm{g}$ titers were significant determinants of $\operatorname{logHOMA}-\mathrm{IR}$ levels $(\beta=-4.38 \mathrm{E}-6 ; p=0.043)$. For HbA1c, both MDA-IgM levels $(\beta=-4.84 E-6 ; p=0.043)$ and total IgM levels $(\beta=-5.508 E-5$; $p=0.011$ ) were significant determinants. These data indicate that antibodies against OxLDL might also be involved in early aberrations in glucose metabolism in children with overweight and obesity.

OSE-specific antibodies are not associated with parameters reflecting cardiovascular health

Lastly, to determine whether the IgM antibodies were also determinants of parameters reflecting cardiovascular health in children, similar regression analyses were performed.

There was an inverse association between MDA-IgM and ICAM levels ( $\beta=-15.573$; $p<0.001)$. For diastolic blood pressure $z$-score, $P 1-\lg M$ levels $(\beta=-2.02 E-5 ; p=0.022)$ were identified as significant inverse determinants (table 4). For all other cardiovascular parameters, the IgM antibodies were not significantly associated, suggesting that antibodies against OSE on oxLDL are not involved in the early cardiovascular changes in children with overweight and obesity. 


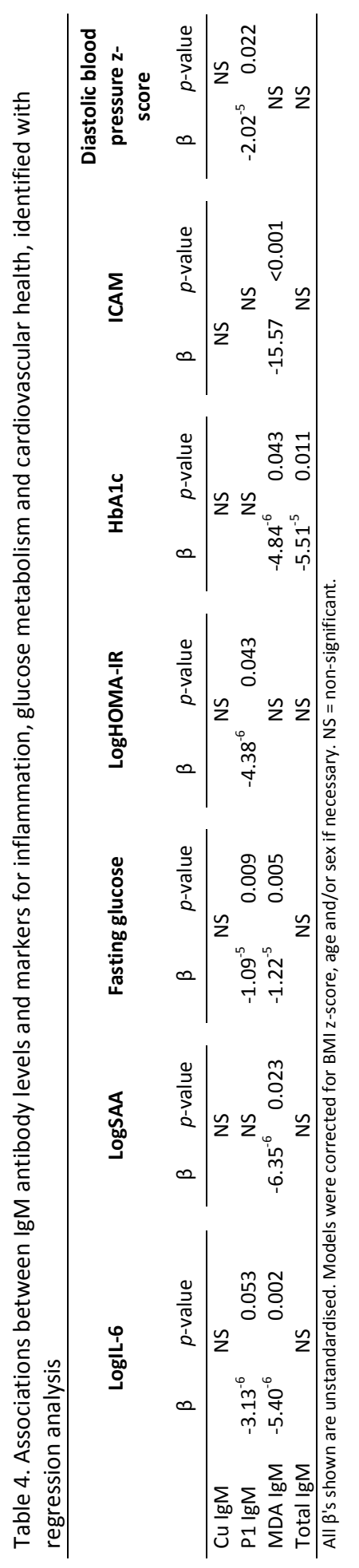




\section{Discussion}

In adults, lower levels of IgM antibodies against oxidized LDL have been associated with the development of non-alcoholic fatty liver disease (14). However, the role of these antibodies against oxidation specific epitopes on oxLDL particles in the development of NAFLD in children is not yet known. Since the pathophysiology and progression of NAFLD is still incompletely understood and previous studies have suggested that underlying processes might differ between children and adults, it is important to also study the potential associations between antibodies against OSE and liver parameters in children.

Our results showed that lower antibody titers against oxLDL were determinants of higher ALT and GGT levels. However, we also showed that the association between OSE-specific antibodies and health parameters was not liver-specific, since there were also associations between lower oxLDL antibodies and markers of low-grade inflammation and parameters reflecting disturbances in glucose metabolism. Interestingly, there were no associations between OSE-specific antibodies and cardiovascular risk parameters.

Previous studies have shown disturbed immune responses to infections and vaccinations (i.e. lower immune cell levels and impaired cell activity) in obese adults in general (15). Furthermore, a recent systematic review of pediatric studies has suggested this is already evident in healthy obese children and children with obesity and asthma (23). Here, we show that IgM levels targeting Cu-oxLDL tended to be lower in obese children and were significantly lower in morbidly obese children, as compared to lean children. Previously, Garoufi et al. did not find an association between total combined IgM and IgG anti-oxLDL antibodies and BMI in children. However, in that study levels of OSE-specific antibodies were not measured and only $17 \%$ of children had overweight or obesity (24). Total IgM levels were not different between lean children and children with overweight or obesity in our study, however there was a correlation between total IgM levels and BMI zscore. Previous studies examining total IgM levels in lean children and children with overweight and obesity have shown conflicting results. Bassols et al. did not find a difference in total IgM levels between lean children and children with overweight or obesity (25). However, a study by Okamatsu et al. did find lower total IgM concentrations in children with overweight or obesity compared to lean children (26). Additional studies in pediatric cohorts are needed the further elucidate the association between (morbid) obesity and OSE-specific antibody levels in children.

The main finding from this study is that we found an association between circulating concentrations of OSE-specific antibodies and parameters of liver health. More specific, Cu-oxLDL and P1-IgM levels in children with ALT levels above twice the upper limit of 
normal were lower as compared to children with normal ALT levels and regression models identified a contribution of these lower antibody levels against oxLDL to both elevated ALT and GGT levels. These findings are in line with our previous results showing decreased levels of several OSE-specific IgM antibodies, in particular P1-IgM levels, in adults with NAFLD compared with healthy controls (14). The underlying mechanism of lower IgM antibodies against OSE in NAFLD patients remains to be clarified. Possibly, IgM antibody levels could be lower because of genetic variation or obesity-related factors like changes in the gut microbiome, which can affect B-cell function (27), and thereby contribute to the development of NAFLD. Otherwise, it is possible that lower IgM antibody titers in NAFLD are a consequence of increased antibody consumption because of increased levels of OSE (14). A previous study by our group in mice showed that NASH severity was reduced after immunization against Streptococcus pneumoniae, which led to increased titers of antibodies against oxLDL based on molecular mimicry (10). That study suggests that antibodies against oxLDL could indeed play a role in the development of NAFLD/NASH. Therefore, based on the available evidence, it is tempting to speculate that lower antibody titers against oxLDL specific epitopes are a cause rather than a consequence of NAFLD in these overweight and obese children. This would then also imply that improving the immune response in overweight or obese populations would lower the risk of developing NAFLD, maybe via an improved IgM immune response towards danger signals like oxLDL. Previously, it was shown in adults that weight loss can normalize aspects of immune function that were aberrant in obesity (28).

Our findings that both MDA and P1-IgM levels were significantly lower in boys compared to girls, combined with the inverse relation between OSE-specific antibody levels and liver enzyme levels, might suggest that boys are at an increased risk of developing NAFLD. This is line with previous studies showing that NAFLD is more prevalent in males $(29,30)$. Previous studies have shown lower combined IgM and IgG anti-oxLDL antibodies (24), as well as lower total IgM concentrations (31) in boys compared to girls, however OSEspecific antibody levels were not measured in those studies. It was previously shown that the production of IgM and IgG antibodies is increased under the influence of estrogens and decreased under the influence of testosterone (32), contributing to this sex difference in antibody levels. Additional studies are needed to replicate and further elucidate these findings, which can help in understanding etiological differences in the development of NAFLD and metabolic aberrations between boys and girls.

In order to investigate if associations between OSE-specific antibodies and health outcomes were liver-specific, we also analyzed elaborate parameters reflecting glucose 
metabolism, systemic inflammation and cardiovascular health. These analyses identified MDA-IgM and P1-IgM as determinants of fasting glucose levels, HOMA-IR and HbA1c. A previous study found lower MDA-IgG antibody titers, and a trend towards lower MDA-IgM antibody titers, in adults with impaired glucose tolerance or diabetes (33). In our population of overweight and obese children MDA-IgM antibody titers were also identified as significant determinants of logSAA levels and logIL-6 levels. Also, there was a trend towards an association between P1-IgM titers and log IL-6 levels. Previously, we also found an inverse relation between P1-IgM titers and IL-6 levels in adults (14). So, our current observations regarding the association between low OSE-specific antibodies and a disturbed glucose metabolism and low-grade inflammation in children, confirm previous associations that were shown in adults.

Regarding cardiovascular risk parameters, regression analysis identified only MDA-IgM as a significant determinant of sICAM-1 levels and P1-IgM as a determinant of diastolic blood pressure z-score, but similar patterns were not seen for the other antibodies or cardiovascular health parameters. It is interesting to consider why we did not find a clear association between IgM antibodies against oxLDL and parameters for cardiovascular health. The association between oxidized LDL cholesterol and cardiovascular disease is well-established and previous studies have shown that cardiovascular health parameters can already be aberrant in children with obesity $(34,35)$. The lack of associations could be explained by the fact that the vascular aberrations seen in our pediatric population are too early in the CVD development trajectory to detect associations with antibodies against oxLDL and as such explain the discordance with observations in adults. Further studies are needed to validate these findings regarding the association between antibodies against oxLDL and cardiovascular health in children.

\section{Conclusion}

Low titers of IgM antibodies against OSE on oxLDL are associated with higher liver enzyme levels in obese children, suggesting that low OSE-specific antibodies might play a role in the etiology of disturbed liver function. However, further studies are needed to elucidate whether the associations with IgM antibodies against OSE are liver-specific, since low OSEspecific antibodies were also related to the presence of systemic inflammation and an unfavorable glucose metabolism, but not with cardiovascular parameters. 
Chapter 8

\section{Acknowledgements}

We kindly thank Dr. Christoph Binder for supplying the materials that were used for performing the antibody measurements. 


\section{References}

1. Ashraf, N.U. and T.A. Sheikh, Endoplasmic reticulum stress and Oxidative stress in the pathogenesis of Non-alcoholic fatty liver disease. Free Radic Res, 2015. 49(12): p. 1405-18.

2. Rehman, K. and M.S.H. Akash, Mechanism of Generation of Oxidative Stress and Pathophysiology of Type 2 Diabetes Mellitus: How Are They Interlinked? J Cell Biochem, 2017. 118(11): p. 3577-3585.

3. Kattoor, A.J., et al., Oxidative Stress in Atherosclerosis. Curr Atheroscler Rep, 2017. 19(11): p. 42.

4. Du, T., X. Sun, and X. Yu, Non-HDL cholesterol and LDL cholesterol in the dyslipidemic classification in patients with nonalcoholic fatty liver disease. Lipids Health Dis, 2017. 16(1): p. 229.

5. Fernandez-Friera, L., et al., Normal LDL-Cholesterol Levels Are Associated With Subclinical Atherosclerosis in the Absence of Risk Factors. J Am Coll Cardiol, 2017. 70(24): p. 29792991.

6. Steinberg, D. and J.L. Witztum, Oxidized low-density lipoprotein and atherosclerosis. Arterioscler Thromb Vasc Biol, 2010. 30(12): p. 2311-6.

7. Binder, C.J., et al., The role of natural antibodies in atherogenesis. J Lipid Res, 2005. 46(7): p. 1353-63.

8. Yimin, et al., A novel murine model for non-alcoholic steatohepatitis developed by combination of a high-fat diet and oxidized low-density lipoprotein. Lab Invest, 2012. 92(2): p. 265-81.

9. Bieghs, V., et al., Trapping of oxidized LDL in lysosomes of Kupffer cells is a trigger for hepatic inflammation. Liver Int, 2013. 33(7): p. 1056-61.

10. Bieghs, V., et al., Specific immunization strategies against oxidized low-density lipoprotein: a novel way to reduce nonalcoholic steatohepatitis in mice. Hepatology, 2012. 56(3): p. 894-903.

11. Calcaterra, V., et al., Relation between circulating oxidized-LDL and metabolic syndrome in children with obesity: the role of hypertriglyceridemic waist phenotype. J Pediatr Endocrinol Metab, 2017. 30(12): p. 1257-1263.

12. Morell-Azanza, L., et al., Serum oxidized low-density lipoprotein levels are related to cardiometabolic risk and decreased after a weight loss treatment in obese children and adolescents. Pediatr Diabetes, 2017. 18(5): p. 392-398.

13. Binder, C.J., Natural IgM antibodies against oxidation-specific epitopes. J Clin Immunol, 2010. 30 Suppl 1: p. S56-60.

14. Hendrikx, T., et al., Low levels of IgM antibodies recognizing oxidation-specific epitopes are associated with human non-alcoholic fatty liver disease. BMC Med, 2016. 14(1): p. 107.

15. Milner, J.J. and M.A. Beck, The impact of obesity on the immune response to infection. Proc Nutr Soc, 2012. 71(2): p. 298-306.

16. Rijks, J.M., et al., Children With Morbid Obesity Benefit Equally as Children With Overweight and Obesity From an Ongoing Care Program. J Clin Endocrinol Metab, 2015. 100(9): p. 3572-80.

17. Amir, S., et al., Peptide mimotopes of malondialdehyde epitopes for clinical applications in cardiovascular disease. J Lipid Res, 2012. 53(7): p. 1316-26.

18. Cole, T.J. and T. Lobstein, Extended international (IOTF) body mass index cut-offs for thinness, overweight and obesity. Pediatr Obes, 2012. 7(4): p. 284-94.

19. Schwimmer, J.B., et al., SAFETY study: alanine aminotransferase cutoff values are set too high for reliable detection of pediatric chronic liver disease. Gastroenterology, 2010. 138(4): p. 1357-64, 1364.e1-2. 
20. Friedewald, W.T., R.I. Levy, and D.S. Fredrickson, Estimation of the concentration of lowdensity lipoprotein cholesterol in plasma, without use of the preparative ultracentrifuge. Clin Chem, 1972. 18(6): p. 499-502.

21. Wuhl, E., et al., Distribution of 24-h ambulatory blood pressure in children: normalized reference values and role of body dimensions. J Hypertens, 2002. 20(10): p. 1995-2007.

22. Joris, P.J., et al., Diet-induced weight loss improves not only cardiometabolic risk markers but also markers of vascular function: a randomized controlled trial in abdominally obese men. Am J Clin Nutr, 2017. 105(1): p. 23-31.

23. Kelishadi, R., et al., Association of Childhood Obesity and the Immune System: A Systematic Review of Reviews. Child Obes, 2017. 13(4): p. 332-346.

24. Garoufi, A., et al., Influence of hypercholesterolemia on serum antibodies against oxidized LDL in children and adolescents. Pediatr Int, 2018. 60(3): p. 287-293.

25. Bassols, J., et al., Increased serum IgG and IgA in overweight children relate to a less favourable metabolic phenotype. Pediatr Obes, 2014. 9(3): p. 232-8.

26. Okamatsu, Y., et al., Ghrelin and leptin modulate immunity and liver function in overweight children. Pediatr Int, 2009. 51(1): p. 9-13.

27. Petta, I., et al., Interrelation of Diet, Gut Microbiome, and Autoantibody Production. Front Immunol, 2018. 9: p. 439.

28. Fontana, L., et al., Impaired mononuclear cell immune function in extreme obesity is corrected by weight loss. Rejuvenation Res, 2007. 10(1): p. 41-6.

29. Anderson, E.L., et al., The Prevalence of Non-Alcoholic Fatty Liver Disease in Children and Adolescents: A Systematic Review and Meta-Analysis. PLoS One, 2015. 10(10): p. e0140908.

30. Wang, Z., et al., Sex-specific prevalence of fatty liver disease and associated metabolic factors in Wuhan, south central China. Eur J Gastroenterol Hepatol, 2014. 26(9): p. 101521.

31. Stoop, J.W., et al., Serum immunoglobulin levels in healthy children and adults. Clin Exp Immunol, 1969. 4(1): p. 101-12.

32. Oertelt-Prigione, S., The influence of sex and gender on the immune response. Autoimmun Rev, 2012. 11(6-7): p. A479-85.

33. Garrido-Sanchez, L., et al., Reduced levels of anti-MDA LDL antibodies in patients with carbohydrate metabolism disorders. Clin Lab, 2011. 57(11-12): p. 901-7.

34. Rijks, J., et al., Characteristics of the retinal microvasculature in association with cardiovascular risk markers in children with overweight, obesity and morbid obesity. Sci Rep, 2018. 8(1): p. 16952.

35. Skinner, A.C., et al., Cardiometabolic Risks and Severity of Obesity in Children and Young Adults. N Engl J Med, 2015. 373(14): p. 1307-17. 


\section{Chapter 9}

General discussion 


\section{Overview}

The Global Burden of Disease study estimated that in 2015, 108 million children and 604 million adults worldwide had obesity (1). This study also showed that the prevalence of obesity has doubled since 1980 in 73 countries worldwide and that the rate of increase in obesity in children was even higher than in adults (1). In the Netherlands, the prevalence of overweight in children aged 4 to 17 years in 2018 was $8.9 \%$ in boys and $9.1 \%$ in girls, while the prevalence of obesity was $2.5 \%$ in boys and $3.0 \%$ in girls (2). Lobstein et al. have predicted that in 2025 , unless we can have a larger impact on childhood obesity in the years until then, around half of the children with obesity will experience one or more comorbidities, like type 2 diabetes, hypertension or non-alcoholic fatty liver disease (3).

In the studies in this dissertation we have focused on the comorbidities of overweight and obesity in children in general, with a special focus on nonalcoholic fatty liver disease (NAFLD). The term NAFLD encompasses a spectrum of liver disorders ranging from hepatic steatosis, to steatohepatitis and liver fibrosis and cirrhosis. NAFLD is a common chronic liver disease in children and adults with obesity $(4,5)$. Currently, the underlying pathophysiological mechanisms of NAFLD development and progression in children are still incompletely understood.

The aims of the studies in this dissertation were 1) to assess the health consequences of overweight and obesity in children, 2) to gain more insight into determinants and risk factors of hepatic steatosis and steatohepatitis in children with overweight and obesity, and 3) to assess the effects of one year of the $\mathrm{COACH}$ lifestyle intervention on body mass index (BMI) z-score and comorbidities. This chapter summarizes the major findings of the studies presented in this thesis, discusses these results in a broader perspective and offers future perspectives.

\section{Consequences of childhood overweight and obesity}

Obesity during childhood is an important risk factor for the development of various comorbidities including dyslipidemia, hypertension, diabetes, sleep apnea, kidney disease, non-alcoholic fatty liver disease, musculoskeletal problems and psychological problems (612). In chapter $\mathbf{2}$, we have shown that comorbidities of obesity were already common at primary school age. For instance, insulin resistance was present in $37 \%$, dyslipidemia in 
$48 \%$, hypertension in $7 \%$ and elevated liver transaminase levels in $54 \%$ of primary school children. Interestingly, these comorbidities were mostly already as common in primary school children compared to secondary school children. The prevalence of comorbidities that we found were comparable to those described in a large European pediatric population (13). Noteworthy differences between our findings and other studies mainly concern differences in glucose metabolism abnormalities. Whereas we found a very low prevalence of impaired fasting glucose (1\%) and type II diabetes $(0.4 \%)$, a large study in teenagers from the United States found an impaired fasting glucose prevalence of $23 \%$ (6). These differences could be the consequence of ethnical differences between the study populations (14) or differences in the degree of obesity. A direct comparison of the degree of obesity is impaired by the use of different BMI classifications between studies. Further, differences in dietary and physical activity habits might also contribute to the difference in the prevalence of glucose metabolism abnormalities, since it was previously shown that diet quality and lack of physical activity were associated with an increased risk of developing diabetes even after adjusting for $\operatorname{BMI}(15,16)$.

In addition to the general cardiovascular parameters that were studied in chapter 2, we have focused on the augmentation index (AIX), as a marker for arterial stiffness in chapter 4. In adults, increased arterial stiffness has been clearly associated with future cardiovascular disease risk (17). Previous studies in children have also shown an association between the Alx and dyslipidemia (18) and type 2 diabetes (19), however the predictive value of the Alx for future cardiovascular disease in children remains to be determined. As it is not yet fully known what the determinants of the Alx in children are, we evaluated the associations between the augmentation index and cardiometabolic risk parameters and markers reflecting inflammation and endothelial dysfunction. In our study, BMI z-score was not associated with the Alx in children with overweight or (morbid) obesity. Lean children were not included in this study. Previous studies have shown conflicting results regarding the association between the Alx and overweight or obesity. A meta-analysis by Hudson et al. did not find a difference in the Alx between children with obesity compared to non-obese children after pooling of the available data (20). Lentferink et al. also did not find a higher Alx in obese adolescents compared to lean adolescents (21). It was previously suggested that these findings could be the result of dilatation of the arteries in people with obesity (22).

Interestingly, the Alx was higher in girls than in boys, which is in line with previous studies in both children and adults that also found a higher Alx in women (23-27). Since height is an important predictor of the Alx, the height difference between boys and girls partially explained the higher Alx in girls that we found in chapter 4. However, a sex difference was 
still present after correcting for height, which is also in line with a previous study that found a difference in the Alx in boys and girls that were matched for height (23). In that study, a higher Alx was already seen in girls before puberty, just like in our study, demonstrating that hormonal differences probably do not play an important role in the sex difference in Alx. Previous studies in adults have suggested that differences in pulse wave morphology and smaller and stiffer blood vessels in women compared to men, play a role in the sex difference in the $\operatorname{Alx}(25,28)$.

In addition to the sex difference in Alx, we also found that the determinants of the Alx differed between boys and girls with overweight and obesity. In boys, height and the markers for inflammation and endothelial dysfunction were identified as determinants of the Alx, compared to height and diastolic blood pressure in girls. Previously, studies in adults also described a different contribution of metabolic and inflammatory parameters to the Alx in men compared to women $(26,27)$. Additional studies are needed to further explore the factors that underlie the sex difference in arterial stiffness that we and others found and how these findings might influence intervention strategies.

\section{Non-alcoholic fatty liver disease}

Non-alcoholic fatty liver disease is the most common chronic liver disease in the developed world (29). The global prevalence of hepatic steatosis in the general adult population was estimated to be $25 \%$ (30). The prevalence of steatohepatitis was estimated to be $1.5-6.5 \%$, depending on the indication for liver biopsy (30). Even at pediatric age, hepatic steatosis is prevalent in $8 \%$ of the general population and in $34 \%$ of children treated in obesity clinics (4). NAFLD can have severe consequences for the liver itself, as the disease can progress to liver cirrhosis and possibly necessitate a liver transplant (31). In the United States, NAFLD-related liver cirrhosis is the most rapidly increasing cause of a liver transplant (32). Also, NAFLD patients have an increased risk of developing hepatocellular carcinoma (33). Progression to the severe end of the disease spectrum is much more rapid in some patients compared to others. In addition to these possible consequences for the liver, NAFLD also has been associated with increased risk for developing diabetes type II $(34,35)$, chronic kidney disease (35), decreased bone mineral density (35) and cardiovascular disease (36). However, additional studies are needed to examine if these associations are independent from other risk factors for these diseases, like obesity, age and smoking.

It is important that the possible severity of NAFLD is not underestimated. However, a previous Australian study showed that hospital specialists tend to underestimate the 
expected prevalence of NAFLD in their patients and that only $30 \%$ of the specialists would refer a patient suspected to have NAFLD to a hepatologist (37). Similarly, a British study found that NAFLD prevalence and severity was likely to be underestimated by diabetes specialists (38).

Previous studies have shown histological differences in characteristics of NAFLD in children compared to adults, suggesting that underlying pathophysiological processes might also differ between children and adults. Therefore, it is important to study NAFLD development and progression not only in adults, but also in children. A more elaborate understanding of the determinants of progressive NAFLD in children can contribute to a better clinical recognition of children that are at-risk for progressive liver disease and can help in identifying new targets for the treatment of NAFLD.

\section{Determinants of (progressive) NAFLD}

In 1998, Day et al. proposed the 'two-hit'-hypothesis as an explanation for the pathogenesis of NAFLD/NASH (39). According to this hypothesis, fat accumulation in the liver is the 'first hit', causing hepatic steatosis. Progression to steatohepatitis was thought to subsequently occur as a consequence of a 'second hit', like oxidative stress or an increased secretion of pro-inflammatory cytokines $(39,40)$. However, ongoing studies towards NAFLD pathophysiology showed that the underlying processes are more complex, leading to the formulation of the 'multiple-hit'-hypothesis. According to this hypothesis, multiple factors including genetic predisposition, insulin resistance, oxidative stress and gut microbiota, act synergistically in the development and progression of NAFLD (41).

In chapter 5, we have reviewed the current literature on factors that contribute to the development and progression of NAFLD in children and adults. Figure $1 \mathrm{~A}$ in chapter 5 gives an overview of processes underlying steatosis development based on current literature. Fatty acids, derived from dietary intake, adipose tissue lipolysis and de novo lipogenesis in the liver, can accumulate in the liver, being stored as triglycerides in the hepatocytes. Increased insulin levels contribute to hepatic steatosis by increasing lipogenesis.

In chapter 6, we evaluated determinants of hepatic fat content, as measured with the ultrasonographic hepatorenal index (HRI), in children with overweight, obesity and morbid obesity. In the study described in this chapter, ALT levels, fasting glucose levels and CRP levels were associated with liver fat content. Additionally, we found a trend towards a 
higher percentage of girls in the children with a low HRI and a higher percentage of boys in children with a high HRI. Interestingly, we did not find a correlation between the hepatorenal index and metabolic aberrations or BMI z-score. Comparison with previous data is difficult, since our study was the first to study associations between the HRI and anthropometric and cardiometabolic parameters in children. A previous study in children that measured liver fat content with ${ }^{1} \mathrm{H}$-MRS found an association between liver fat content and components of the metabolic syndrome in adolescent girls with obesity, but not in younger girls or in boys (42). These findings suggest that other parameters besides the ones evaluated in this study are important determinants of liver fat content, especially since our results showed that we can only predict around $26 \%$ of HRI variance with age, sex and anthropometric and metabolic parameters. It is known from previous studies that genetic predisposition plays an important role in the development and progression of NAFLD (43-46), which will be elaborated further later on in this discussion. Diet quality might also be a determinant of hepatic steatosis. As was also discussed in chapter $\mathbf{5}$, a high carbohydrate intake, in particular a high fructose intake, has been linked to increased insulin resistance, increased lipogenesis and decreased ?-oxidation of fatty acids, contributing to an increased fatty acid influx into the liver and thereby steatosis. Moreover, higher concentrations of vitamin $\mathrm{E}$ have also been associated with decreased hepatic steatosis (47). However, further research regarding specific dietary components and hepatic steatosis is needed, since previous studies have shown conflicting results.

Even though studies during the past decades have provided increased insight into the factors that contribute to the progression along the disease spectrum, it is still not completely understood which patients are at risk for a fast progression to steatohepatitis and liver fibrosis. A review of current literature in chapter $\mathbf{5}$ has summarized several factors that can increase the risk of progression to NASH, including lifestyle and dietary habits, sex, gut microbiome, adipokines, oxidative stress and genetic predisposition. These factors are summarized in figure 1B in chapter 5.

In chapter 6 we showed that the factors that are associated with progression to steatohepatitis, as assessed with ALT concentrations, are not the same as the factors that are associated with the development of hepatic steatosis (summarized in figure 1 from chapter 6). Children with more severe hepatic steatosis also had higher ALT concentrations. Additionally, a higher BMI z-score, higher triglyceride levels, higher ferritin levels and male sex were all associated with progression to steatohepatitis. Moreover, in chapter 7 we found that children with a genetic predisposition (i.e. carrying a PNPLA3 and/or TM6SF2 risk allele), higher total cholesterol levels and a higher degree of insulin resistance have higher ALT concentrations. 


\section{Genetic determinants of NAFLD}

In the past decade, it has become increasingly clear that genetic predisposition plays an important role in the development and progression of NAFLD. Of these genetic variants, the I148M-variant of the patatin-like phospholipase domain containing 3 (PNPLA3) has repeatedly been shown to be a very important determinant for the presence and severity of steatosis $(43,44,48)$, but also fibrosis (48). An increased liver fat content in PNPLA3 G allele carriers was already seen in children under the age of 10 years (49). A meta-analysis of studies mostly performed in adults estimated an odds ratio (OR) of 3.41 for the development of NAFLD and an OR of 4.44 for development of NASH in carriers of the PNPLA3 variant (50). Moreover, PNPLA3 GG homozygotes have a five-fold increased risk of developing hepatocellular carcinoma compared to non-risk carriers (51).

The PNPLA3 gene encodes for the adiponutrin enzyme, which is primarily expressed in hepatocytes (52). The current hypothesis is that the risk variant leads to triglyceride accumulation in the liver because the PNPLA3-148M protein accumulates on lipid droplets and thereby impairs the mobilization of triglycerides from these droplets (53).

Another genetic variant that has been associated with an increased risk of developing NAFLD is the E167K variant of the transmembrane 6 superfamily member 2 (TM6SF2). A meta-analysis of studies in adults estimated that carrying this risk variant leads to an OR of 2.13 for the development of steatosis (46). Dongiovanni et al. found an OR of 1.84 for the development of NASH and an OR of 2.08 for development of fibrosis grade 3 or 4 in a cohort of adults in which liver biopsies were performed (54). The presence of the risk allele reduces TM6SF2 function, leading to decreased secretion of very-low-densitylipoproteins (VLDL) from the liver (55), thereby increasing hepatic triglyceride content. Adults carrying the TM6SF2 variant were shown to have lower serum total cholesterol, LDL cholesterol and triglyceride concentrations (46) and had a decreased risk of developing atherosclerosis, with an OR of 0.48 (54).

Previous studies towards the association between these genetic variants have focused mainly on evaluating these SNPs as risk factors for NAFLD in various populations (e.g. adults, children or different ethnicities). Since the proteins that these genes encode for are involved in processes related to the development and progression of NAFLD, it can be hypothesized that the determinants of NAFLD differ between genetic variants. In chapter 7, we have shown that this was indeed the case. Carrying the PNPLA3 and/or the TM6SF2 risk allele was a significant determinant of NAFLD in the total group of children, in addition to male sex and metabolic aberrations. Stratification for genotype showed that triglyceride levels were only associated with ALT levels in children carrying at least one 
PNPLA3 risk allele. Interestingly, even though triglyceride levels and HOMA-IR were identified as significant predictors of ALT levels in children carrying a PNPLA3 G allele, these children did not have elevated triglyceride levels or HOMA-IR, compared to children without a PNPLA3 risk allele. These results could suggest that there is a difference in sensitivity for the possible harmful effects in the liver of PNPLA3 G allele carriers. In TM6SF2 non-risk carriers, male sex, total cholesterol levels, triglyceride levels and HOMAIR were associated with higher ALT levels, but not in carriers of the TM6SF2 risk allele.

These results demonstrate that the factors involved in the development of NAFLD can differ between people with a different genetic background and cannot simply be generalized across populations. These differences are important to take into consideration from a clinical point of view, as we have demonstrated that high ALT levels can already be present with normal metabolic determinants in at-risk populations. It is also important to take these results into account in further scientific studies towards NAFLD, as the results might also not be generalized across populations.

The role of genetic predisposition in the development of NAFLD is a very active field of research at this time. Recently, additional SNPs associated with NAFLD besides the one mentioned previously in this dissertation have been identified, including variants in the glucokinase regulator (GCKR) (56) and in the membrane bound O-acyltransferase domaincontaining 7 gene (MBOAT7) gene $(57,58)$. The GCKR variant contributes to hepatic steatosis by increasing malonyl-CoA concentrations, which then serve a substrate for lipogenesis and impair fatty acid oxidation (59). The MBOAT7 variant decreased protein activity and leads to the accumulation of lipid droplets in the liver (60). Last year, AbulHusn et al. described a variant in hydroxysteroid 17-beta dehydrogenase 13 (HSD17B13) that was associated with a lower risk of steatohepatitis, fibrosis and hepatocellular carcinoma (61), thereby protecting against progressive liver disease. The authors found an interaction between this variant and the PNPLA3 variant, in which the unfavorable effect of the PNPLA3 gene was attenuated by the HSD17B13 (61). The function of HSD17B13 remains to be fully elucidated. Further studies are needed to validate these recently discovered SNPs in pediatric cohorts. Since we have seen in chapter 7 that determinants of ALT levels can differ between genotypes, it is important to assess if this is also true for the GCKR, MBOAT7 and HSD17B13 variants.

Previous studies have shown important gene-gene and gene-environment interactions between the aforementioned variants and other NAFLD risk factors. For instance, a study by Koo et al. found that the PNPLA3 and TM6SF2 risk alleles additively increase the risk of NASH (62). Moreover, Stender et al. reported that the increasing effect of the PNPLA3, 
TM6SF2 and GCKR on hepatic triglyceride content was mainly present in adults with (severe) obesity and to a lesser extent in adults with a normal weight (63). A combination of these genetic variants and other determinants of NAFLD, might be used to create a risk score for early identification of people with a high risk of progressive liver disease. Further studies are needed to examine which combination of parameters would have the best predictive value and validation in different populations would be necessary.

Moreover, the genetic variants might also be used to personalize intervention strategies. A study by $\mathrm{Ma}$ et al. has shown that increasing diet quality was especially important for decreasing liver fat content in adults with a genetic NAFLD risk score (64). Another study found an interaction between the PNPLA3 polymorphism and physical activity and sedentary behavior in children (65). Finally, further insight into the underlying mechanisms by which these genetic variants contribute to hepatic steatosis, NASH and fibrosis, can help in identifying new targets for medical treatment options.

\section{Oxidative stress as a determinant of NAFLD}

Oxidative stress has been associated with the pathophysiology of NASH. Lipid accumulation in the liver leads to $\beta$-oxidation of fatty acids in mitochondria. Continuous excessive influx of fatty acids into the liver can exceed the capacity of the mitochondria, which leads to the formation of reactive oxygen species (66). Oxidative stress in the liver can contribute to hepatocellular injury and stellate cell activation (chapter 5).

Oxidative stress can also lead to the formation of oxidized low-density lipoproteins (oxLDL). These oxLDL particles get internalized by scavenger cells of the immune system, including Kuppfer cells leading to the formation of pro-inflammatory foam cells. OxLDL particles have been clearly associated with an increased risk of cardiovascular disease in adults $(67,68)$, but have also been suggested to contribute to NAFLD (69-71). A possible association between oxLDL and the development and/or progression of NAFLD in children remains to be studied.

Natural immunoglobulin M (IgM) antibodies can bind to oxidation-specific epitopes that are present on oxLDL particles and protect against the harmful effects by blocking the formation of pro-inflammatory foam cells $(68,72)$. In chapter 8 , we evaluated for the first time in children, associations between antibodies against oxidation specific epitopes on oxLDL and parameters reflecting NAFLD. In line with previous findings in adults (73), we found that low titers of antibodies against OSE on oxLDL were associated with higher liver enzyme levels in obese children, suggesting that low OSE-specific IgM antibodies might play a role in the etiology of disturbed liver health. In adults, low levels of IgM antibodies against oxLDL have been associated with a higher risk of atherosclerosis $(74,75)$, but 
associations between the OSE-specific antibodies and cardiovascular parameters were not present in our pediatric populations. As this was the first study towards the association between IgM antibodies against OSE and NAFLD in children, additional studies are needed to replicate and further elucidate these findings. In the future there might also be a therapeutic use for these antibodies against oxLDL, as animal studies have shown that an increase in antibodies after pneumococcal vaccination can improve atherosclerosis and NASH $(71,76)$. Moreover, observational studies in adults have also suggested a protective effect of pneumococcal vaccination against acute coronary syndrome (77) and a randomized controlled trial is ongoing (78).

\section{Sex as a determinant of NAFLD}

Between pubertal and menopausal age, NAFLD is more common in males than in females (79). Denzer et al. found a prevalence of hepatic steatosis $51 \%$ in postpubertal boys, compared to $12 \%$ in postpubertal girls (80). Our results from chapters 6 and 7, also identified male sex as a determinant of ALT levels. A review of current literature in chapter $\mathbf{5}$ of this thesis suggests that hormones might play an important role in this sex difference. Estrogens were shown to modulate lipid metabolism and have antiinflammatory effects, thereby protecting against NASH (81). Higher testosterone levels were associated with insulin resistance in adolescent boys with obesity (82). Studies examining associations between sex hormones and NAFLD in children before, during and after puberty are needed to further elucidate these findings. Additionally, a previous study in adults has shown that women seem protected against the negative effects of fructose intake (83), which is considered an important risk factor for NAFLD (84). Finally, levels of IgM antibodies against OSE on oxidized LDL were also lower in boys than in girls (chapter 8). The production of IgM antibodies is increased under the influence of estrogen and decreased under the influence of testosterone (85), contributing to the sex difference in antibody levels. Since the antibodies against OSE are suggested to be protective against NAFLD, the lower concentrations that we found in boys might be one of the factors contributing to a higher NAFLD prevalence in boys.

\section{Interventions in children with overweight or obesity}

The clinical practice guideline for the treatment of NAFLD in children of the North American Society of Pediatric Gastroenterology, Hepatology and Nutrition recommends lifestyle improvements as the primary treatment option, including increasing physical 
activity and avoidance of sugar-sweetened beverage to decrease obesity (86). Medication or nutritional supplements are not recommended as part of NAFLD treatment according to this guideline, because none have been proven to benefit a majority of children with NAFLD. The authors of this guidelines also conclude that from the available data, evidence is limited to advice a certain amount of weight or BMI improvement that is necessary to decrease NAFLD/NASH in children. A study in adults showed resolution of NASH in $90 \%$ of the patients that lost $10 \%$ of their body weight or more. Liver fat content decreased in all patients that lost at least $10 \%$ of their body weight (87). Relevantly, several reviews, in both children and adults, have shown that physical activity (88) or combined lifestyle interventions (89) can have an beneficial effect on NAFLD, even without significant weight loss.

However, in current society, living a healthier lifestyle and losing weight can be a difficult goal to achieve, especially for children. Unhealthy products and services promoting an unhealthy lifestyle are widely available and sometimes more easily accessible than healthy options. Changing this environment to a situation in which healthy options are accessible and affordable for everyone, will require a combined effort from stakeholders across several levels of society, for instance health care professionals and (local) governments, but also schools, sports clubs and supermarkets. For children that are already overweight or obese, there is a need for lifestyle interventions that help them and their families in making small, realistic changes in their lifestyle that can be maintained on the long-term. These interventions also require an interdisciplinary approach that can be adapted based on the needs of the children and their family.

In chapter 2, we evaluated the effect of one year of the $\mathrm{COACH}$ interdisciplinary lifestyle intervention on elaborate health outcomes. We found a significant decrease in BMI zscore after intervention, that was greater in primary school children compared to secondary school children, which is in line with previous studies that also found a larger effect on BMI z-score in younger children (90-92). Additionally, we found a greater improvement of LDL cholesterol concentrations and systolic blood pressure z-score in primary school children, compared to secondary school children. Since previous studies towards differences in the effects of lifestyle interventions between young children and adolescents have focused mainly on the effect on BMI (z-score), comparison of our results regarding effects on health aberrations between age groups is limited. Our findings underline the need for preventive initiatives to decrease the development of obesity and the accompanying health effects and the need to initiate interventions as soon as possible in children that already have obesity.

A distinction between boys and girls in multidisciplinary lifestyle intervention studies is hardly made, but previous studies have described sex differences in general physical 
activity levels (93) in children and the ability to improve several aspects of physical activity at the same time (94). After one year of $\mathrm{COACH}$ lifestyle intervention, we found a significant decrease in BMI z-score in boys, whereas BMI z-score stabilized in girls. However, the changes in health outcomes were comparable between boys and girls. It is possible that the amount of BMI z-score decrease that is associated with clinically relevant changes in health outcomes differs between boys and girls. Differences between the effectiveness of (different components of) lifestyle interventions between boys and girls should be taken into account in future studies, as this might also affect focus points that are addressed in lifestyle interventions.

In chapter 3, we have focused in particular on the effect of one year of the COACH lifestyle intervention on glycaemic profiles measured in free-living conditions in a small group of children with overweight and obesity. It was previously shown in a study in the $\mathrm{COACH}$ cohort that hypo- and hyperglycaemic excursions were already prevalent in these children (95). Also, previous studies in adults have shown that a high frequency and amplitude of glucose fluctuations (i.e. a high glycaemic variability) can have negative effects on vascular function, by contributing to oxidative stress and the secretion of pro-inflammatory cytokines $(96,97)$.

In the study described in chapter $\mathbf{3}$, glucose concentrations were measured during 48 hours, while children were in free-living conditions. Measuring glucose concentrations for a longer period of time, with short intervals, makes it possible to detect subtle changes in glucose metabolism that might be missed with clinical measurements like an oral glucose tolerance test. Glycaemic variability was estimated with the continuous overlapping net glycaemic action (CONGA). The results of this study showed that median sensor glucose concentrations did not change significantly after one year of lifestyle intervention. However, the duration of high-normal glucose concentrations (i.e. glucose $\geq 6.7 \mathrm{mmol} / \mathrm{L}$ ) and glycaemic variability as assessed with CONGA1 decreased significantly during the intervention. Stratification for a decrease or increase in BMI z-score after one year of lifestyle intervention showed that a decrease in BMI z-score was associated with a decrease in glycaemic variability. In these children, a decrease in median sensor glucose was associated with a decrease in LDL-cholesterol concentrations, systolic blood pressure z-score and diastolic blood pressure z-score. Even though the sample size in this study was rather small, the results provide insight in subtle change in free-living glycaemic profiles after lifestyle intervention and the association with changes in cardiovascular risk parameters. Studies towards free-living glycaemic profiles in children without diabetes and the effect of an intervention are limited, impairing a comparison with other populations. Further studies with a larger sample size are needed to gain more insight into the effect of lifestyle intervention on glycaemic profiles and the associations with other 
health outcomes. Also, the specific components of combined lifestyle interventions, such as dietary habits and physical activity, that contribute to changes in glycaemic profiles remain to be further elucidated.

\section{Overall conclusion and future directions}

In summary, we have shown that the comorbidities of obesity are already common in children from a young age. We have also shown that BMI z-score and comorbidities in children with obesity improve during one year of interdisciplinary lifestyle intervention, with a larger effect in primary school children than in secondary school children.

Moreover, we studied determinants of NAFLD, based on a review of current literature and in clinical studies. We have seen that the determinants of steatosis and steatohepatitis in children with overweight or obesity are not the same, and that determinants of steatohepatitis differ between children with different genetic variants. Furthermore, high ALT levels can be found in children with overweight and a genetic predisposition, even when genotype-specific determinants of ALT levels, are within the normal range.

A more elaborate understanding of the determinants of progressive NAFLD in children remains an important research topic for the future, in order to achieve a better clinical recognition of children that are at-risk for progressive liver disease, or already have developed NAFLD. Moreover, a better understanding of the pathophysiology is needed to help identify new targets for effective treatment of NAFLD, since treatment options are currently limited.

Further studies are needed to evaluate SNPs that have been associated with NAFLD but were not yet studied in children in chapter $\mathbf{7}$ of this dissertation. Since we have shown that the determinants of ALT levels may differ between genetic variants, it is important to evaluate if this is also the case for the other genetic variants. Moreover, additional studies are needed to evaluate if a combined risk score of carrying certain genetic variants and other risk determinants of NAFLD, might be useful in early identification of people at risk for developing progressive liver disease. Additionally, as previous studies have suggested interactions between these genetic variants and the effect of dietary and physical activity interventions, adapting interventions strategies based on the genetic risk scores might also be a future perspective in clinical practice. Finally, further insights in the underlying mechanism by which SNPs contribute to the development and progression of NAFLD can help in identifying new targets for additional 
treatment options, especially necessary for patients with severe disease that do not succeed in making and maintaining (sufficient) lifestyle improvements.

In regard to screening and diagnosis of NAFLD, the hepatorenal index studied in chapter 6 of this dissertation has shown high correlations with liver fat content in adults (98-101), but was not yet studied in children. Reference values for children remain to be determined.

Over the past years, promising results have been shown for the controlled attenuation parameter (CAP) and liver stiffness measurement (LSM) performed with the Fibroscan device in non-invasively diagnosing liver steatosis and fibrosis in adults $(102,103)$. Until now, pediatric studies that examined the LSM and CAP mainly compared these measurements to NAFLD diagnosed with ultrasonography, or correlated CAP and LSM outcomes to biomarkers (104-106). Therefore, before this technique can be used in children in a clinical setting, studies are needed to determine reference values in larger pediatric populations in which these measurements are compared with for instance ${ }^{1} \mathrm{H}$ MRS. A previous study in healthy lean children has suggested that LSM values increase during childhood age (107). Based on this finding, and the known sex difference in NAFLD, it should be investigated if the reference values are age- and/or sex-dependent.

Since imaging modalities often lack the ability to diagnose liver inflammation, other sensitive biomarkers for NASH are still necessary. In the future, lipidomics and metabolomics approaches might help in the non-invasive diagnosis of the different NAFLD disease stages, as previous studies have shown to be able to successfully discriminate NAFLD from normal livers (108), but also discriminate between steatosis and NASH (108110). However, additional studies in both children and adults are necessary before these techniques can be translated to widespread clinical settings. Additionally, these techniques might provide more knowledge on the mechanisms that contribute to NAFLD progression and possible targets for treatment.

An ongoing research initiative that will help in further elucidating causal factors, diagnostic options and the natural history of NAFLD in children is the 'European Paediatric NonAlcoholic Fatty Liver Disease (EU-PNAFLD) registry (111). In this multi-center European collaboration up to 2000 children with NAFLD will be included and elaborate health outcomes and biosamples will be collected. Possible long-term metabolic and hepatic complications will be followed-up for up to 30 years. The ability to study a large population of children across the whole disease spectrum and follow the natural history, will provide an unique insight in NAFLD in children. Another important goal of this registry 
is to facilitate trials towards interventions for NASH in children, in order to improve the care for these children. 


\section{References}

1. Afshin A, Forouzanfar MH, Reitsma MB, Sur P, Estep K, Lee A, et al. Health Effects of Overweight and Obesity in 195 Countries over 25 Years. The New England journal of medicine. 2017;377(1):13-27.

2. National Institute of Public Health and the Environment (Rijksinstituut voor Volksgezondheid en Milieu; RIVM). Website: https://www.volksgezondheidenzorg.info/onderwerp/overgewicht/cijferscontext/huidige-situatie\#!node-overgewicht-kinderen. Accessed: 05/07/2018.

3. Lobstein T, Jackson-Leach R. Planning for the worst: estimates of obesity and comorbidities in school-age children in 2025. Pediatr Obes. 2016;11(5):321-5.

4. Anderson EL, Howe LD, Jones HE, Higgins JP, Lawlor DA, Fraser A. The Prevalence of NonAlcoholic Fatty Liver Disease in Children and Adolescents: A Systematic Review and MetaAnalysis. PloS one. 2015;10(10):e0140908.

5. Le MH, Devaki P, Ha NB, Jun DW, Te HS, Cheung RC, et al. Prevalence of non-alcoholic fatty liver disease and risk factors for advanced fibrosis and mortality in the United States. PloS one. 2017;12(3):e0173499.

6. Skinner AC, Perrin EM, Moss LA, Skelton JA. Cardiometabolic Risks and Severity of Obesity in Children and Young Adults. The New England journal of medicine. 2015;373(14):130717.

7. Perrone J, Hollander JE, De Roos F. Cardiovascular risk factors and atherosclerosis in children and young adults. The New England journal of medicine. 1998;339(15):1083-4.

8. Corey KE, Kaplan LM. Obesity and liver disease: the epidemic of the twenty-first century. Clinics in liver disease. 2014;18(1):1-18.

9. Herouvi D, Karanasios E, Karayianni C, Karavanaki K. Cardiovascular disease in childhood: the role of obesity. Eur J Pediatr. 2013;172(6):721-32.

10. Krul M, van der Wouden JC, Schellevis FG, van Suijlekom-Smit LW, Koes BW. Musculoskeletal problems in overweight and obese children. Annals of family medicine. 2009;7(4):352-6.

11. Andersen IG, Holm JC, Homoe P. Obstructive sleep apnea in children and adolescents with and without obesity. European archives of oto-rhino-laryngology : official journal of the European Federation of Oto-Rhino-Laryngological Societies (EUFOS) : affiliated with the German Society for Oto-Rhino-Laryngology - Head and Neck Surgery. 2019;276(3):871-8.

12. Rankin J, Matthews L, Cobley S, Han A, Sanders R, Wiltshire HD, et al. Psychological consequences of childhood obesity: psychiatric comorbidity and prevention. Adolescent health, medicine and therapeutics. 2016;7:125-46.

13. Reinehr T, Wiegand S, Siegfried W, Keller KM, Widhalm K, I'Allemand D, et al. Comorbidities in overweight children and adolescents: do we treat them effectively? Int J Obes (Lond). 2013;37(4):493-9.

14. Razak F, Anand S, Vuksan V, Davis B, Jacobs R, Teo KK, et al. Ethnic differences in the relationships between obesity and glucose-metabolic abnormalities: a cross-sectional population-based study. Int J Obes (Lond). 2005;29(6):656-67.

15. Hu FB, Manson JE, Stampfer MJ, Colditz G, Liu S, Solomon CG, et al. Diet, lifestyle, and the risk of type 2 diabetes mellitus in women. The New England journal of medicine. 2001;345(11):790-7.

16. de Munter JS, Hu FB, Spiegelman D, Franz M, van Dam RM. Whole grain, bran, and germ intake and risk of type 2 diabetes: a prospective cohort study and systematic review. PLoS medicine. 2007;4(8):e261. 
17. Vlachopoulos C, Aznaouridis K, O'Rourke MF, Safar ME, Baou K, Stefanadis C. Prediction of cardiovascular events and all-cause mortality with central haemodynamics: a systematic review and meta-analysis. European heart journal. 2010;31(15):1865-71.

18. Riggio S, Mandraffino G, Sardo MA, ludicello R, Camarda N, Imbalzano E, et al. Pulse wave velocity and augmentation index, but not intima-media thickness, are early indicators of vascular damage in hypercholesterolemic children. European journal of clinical investigation. 2010;40(3):250-7.

19. Urbina EM, Kimball TR, Khoury PR, Daniels SR, Dolan LM. Increased arterial stiffness is found in adolescents with obesity or obesity-related type 2 diabetes mellitus. J Hypertens. 2010;28(8):1692-8.

20. Hudson LD, Rapala A, Khan T, Williams B, Viner RM. Evidence for contemporary arterial stiffening in obese children and adolescents using pulse wave velocity: A systematic review and meta-analysis. Atherosclerosis. 2015;241(2):376-86.

21. Lentferink YE, Kromwijk LAJ, van der Aa MP, Knibbe CAJ, van der Vorst MMJ. Increased Arterial Stiffness in Adolescents With Obesity. Glob Pediatr Health. 2019;6:2333794x19831297.

22. DeVallance E, Fournier SB, Donley DA, Bonner DE, Lee K, Frisbee JC, et al. Is obesity predictive of cardiovascular dysfunction independent of cardiovascular risk factors? Int J Obes (Lond). 2015;39(2):244-53.

23. Ayer JG, Harmer JA, Marks GB, Avolio A, Celermajer DS. Central arterial pulse wave augmentation is greater in girls than boys, independent of height. J Hypertens. 2010;28(2):306-13.

24. Hayward CS, Kelly RP. Gender-related differences in the central arterial pressure waveform. Journal of the American College of Cardiology. 1997;30(7):1863-71.

25. Torjesen AA, Wang N, Larson MG, Hamburg NM, Vita JA, Levy D, et al. Forward and backward wave morphology and central pressure augmentation in men and women in the Framingham Heart Study. Hypertension (Dallas, Tex : 1979). 2014;64(2):259-65.

26. Kim JY, Park JB, Kim DS, Kim KS, Jeong JW, Park JC, et al. Gender Difference in Arterial Stiffness in a Multicenter Cross-Sectional Study: The Korean Arterial Aging Study (KAAS). Pulse (Basel, Switzerland). 2014;2(1-4):11-7.

27. Shiva Kumar P, Medina-Lezama J, Morey-Vargas O, Zamani P, Bolanos-Salazar JF, Chirinos $D A$, et al. Prospective risk factors for increased central augmentation index in men and women. American journal of hypertension. 2015;28(1):121-6.

28. Gatzka CD, Kingwell BA, Cameron JD, Berry KL, Liang YL, Dewar EM, et al. Gender differences in the timing of arterial wave reflection beyond differences in body height. J Hypertens. 2001;19(12):2197-203.

29. Loomba R, Sirlin CB, Schwimmer JB, Lavine JE. Advances in pediatric nonalcoholic fatty liver disease. Hepatology (Baltimore, Md). 2009;50(4):1282-93.

30. Younossi ZM, Koenig AB, Abdelatif D, Fazel Y, Henry L, Wymer M. Global epidemiology of nonalcoholic fatty liver disease-Meta-analytic assessment of prevalence, incidence, and outcomes. Hepatology (Baltimore, Md). 2016;64(1):73-84.

31. Mikolasevic I, Filipec-Kanizaj T, Mijic M, Jakopcic I, Milic S, Hrstic I, et al. Nonalcoholic fatty liver disease and liver transplantation - Where do we stand? World journal of gastroenterology. 2018;24(14):1491-506.

32. Charlton MR, Burns JM, Pedersen RA, Watt KD, Heimbach JK, Dierkhising RA. Frequency and outcomes of liver transplantation for nonalcoholic steatohepatitis in the United States. Gastroenterology. 2011;141(4):1249-53.

33. White DL, Kanwal F, El-Serag HB. Association between nonalcoholic fatty liver disease and risk for hepatocellular cancer, based on systematic review. Clinical gastroenterology and 
hepatology : the official clinical practice journal of the American Gastroenterological Association. 2012;10(12):1342-59.e2.

34. Valenti L, Bugianesi E, Pajvani U, Targher G. Nonalcoholic fatty liver disease: cause or consequence of type 2 diabetes? Liver international : official journal of the International Association for the Study of the Liver. 2016;36(11):1563-79.

35. Adams LA, Anstee QM, Tilg H, Targher G. Non-alcoholic fatty liver disease and its relationship with cardiovascular disease and other extrahepatic diseases. Gut. 2017;66(6):1138-53.

36. Sookoian S, Pirola CJ. Non-alcoholic fatty liver disease is strongly associated with carotid atherosclerosis: a systematic review. Journal of hepatology. 2008;49(4):600-7.

37. Bergqvist CJ, Skoien R, Horsfall L, Clouston AD, Jonsson JR, Powell EE. Awareness and opinions of non-alcoholic fatty liver disease by hospital specialists. Internal medicine journal. 2013;43(3):247-53.

38. Marjot T, Sbardella E, Moolla A, Hazlehurst JM, Tan GD, Ainsworth M, et al. Prevalence and severity of non-alcoholic fatty liver disease are underestimated in clinical practice: impact of a dedicated screening approach at a large university teaching hospital. Diabetic medicine : a journal of the British Diabetic Association. 2018;35(1):89-98.

39. Day CP, James OF. Steatohepatitis: a tale of two "hits"? Gastroenterology. 1998;114(4):842-5.

40. Takahashi Y, Fukusato T. Pediatric nonalcoholic fatty liver disease: overview with emphasis on histology. World journal of gastroenterology. 2010;16(42):5280-5.

41. Buzzetti E, Pinzani M, Tsochatzis EA. The multiple-hit pathogenesis of non-alcoholic fatty liver disease (NAFLD). Metabolism: clinical and experimental. 2016;65(8):1038-48.

42. Nissen A, Fonvig CE, Chabanova E, Bojsoe C, Trier C, Pedersen O, et al. (1)H-MRS measured ectopic fat in liver and muscle is associated with the metabolic syndrome in Danish girls but not in boys with overweight and obesity. Obesity science \& practice. 2016;2(4):376-84.

43. Romeo S, Kozlitina J, Xing C, Pertsemlidis A, Cox D, Pennacchio LA, et al. Genetic variation in PNPLA3 confers susceptibility to nonalcoholic fatty liver disease. Nature genetics. 2008;40(12):1461-5.

44. Sookoian S, Pirola CJ. Meta-analysis of the influence of I148M variant of patatin-like phospholipase domain containing 3 gene (PNPLA3) on the susceptibility and histological severity of nonalcoholic fatty liver disease. Hepatology (Baltimore, Md). 2011;53(6):188394.

45. Sookoian S, Castano GO, Scian R, Mallardi P, Fernandez Gianotti T, Burgueno AL, et al. Genetic variation in transmembrane 6 superfamily member 2 and the risk of nonalcoholic fatty liver disease and histological disease severity. Hepatology (Baltimore, Md). 2015;61(2):515-25.

46. Pirola CJ, Sookoian S. The dual and opposite role of the TM6SF2-rs58542926 variant in protecting against cardiovascular disease and conferring risk for nonalcoholic fatty liver: $A$ meta-analysis. Hepatology (Baltimore, Md). 2015;62(6):1742-56.

47. Sato K, Gosho M, Yamamoto T, Kobayashi Y, Ishii N, Ohashi T, et al. Vitamin E has a beneficial effect on nonalcoholic fatty liver disease: a meta-analysis of randomized controlled trials. Nutrition (Burbank, Los Angeles County, Calif). 2015;31(7-8):923-30.

48. Rotman $\mathrm{Y}$, Koh C, Zmuda JM, Kleiner DE, Liang TJ. The association of genetic variability in patatin-like phospholipase domain-containing protein 3 (PNPLA3) with histological severity of nonalcoholic fatty liver disease. Hepatology (Baltimore, Md). 2010;52(3):894-903.

49. Goran MI, Walker R, Le KA, Mahurkar S, Vikman S, Davis JN, et al. Effects of PNPLA3 on liver fat and metabolic profile in Hispanic children and adolescents. Diabetes. 2010;59(12):3127-30. 
50. Xu R, Tao A, Zhang S, Deng Y, Chen G. Association between patatin-like phospholipase domain containing 3 gene (PNPLA3) polymorphisms and nonalcoholic fatty liver disease: a HuGE review and meta-analysis. Scientific reports. 2015;5:9284.

51. Liu YL, Patman GL, Leathart JB, Piguet AC, Burt AD, Dufour JF, et al. Carriage of the PNPLA3 rs738409 C > G polymorphism confers an increased risk of non-alcoholic fatty liver disease associated hepatocellular carcinoma. Journal of hepatology. 2014;61(1):75-81.

52. Huang Y, He S, Li JZ, Seo YK, Osborne TF, Cohen JC, et al. A feed-forward loop amplifies nutritional regulation of PNPLA3. Proc Natl Acad Sci U S A. 2010;107(17):7892-7.

53. BasuRay S, Smagris E, Cohen JC, Hobbs HH. The PNPLA3 variant associated with fatty liver disease (I148M) accumulates on lipid droplets by evading ubiquitylation. Hepatology (Baltimore, Md). 2017;66(4):1111-24.

54. Dongiovanni P, Petta S, Maglio C, Fracanzani AL, Pipitone R, Mozzi E, et al. Transmembrane 6 superfamily member 2 gene variant disentangles nonalcoholic steatohepatitis from cardiovascular disease. Hepatology (Baltimore, Md). 2015;61(2):506-14.

55. Chen LZ, Xia HH, Xin YN, Lin ZH, Xuan SY. TM6SF2 E167K Variant, a Novel Genetic Susceptibility Variant, Contributing to Nonalcoholic Fatty Liver Disease. Journal of clinical and translational hepatology. 2015;3(4):265-70.

56. Zain SM, Mohamed Z, Mohamed R. Common variant in the glucokinase regulatory gene rs780094 and risk of nonalcoholic fatty liver disease: a meta-analysis. Journal of gastroenterology and hepatology. 2015;30(1):21-7.

57. Viitasalo A, Eloranta AM, Atalay M, Romeo S, Pihlajamaki J, Lakka TA. Association of MBOAT7 gene variant with plasma ALT levels in children: the PANIC study. Pediatric research. 2016;80(5):651-5.

58. Mancina RM, Dongiovanni P, Petta S, Pingitore P, Meroni M, Rametta R, et al. The MBOAT7-TMC4 Variant rs641738 Increases Risk of Nonalcoholic Fatty Liver Disease in Individuals of European Descent. Gastroenterology. 2016;150(5):1219-30.e6.

59. Valenti L, Alisi A, Nobili V. Unraveling the genetics of fatty liver in obese children: additive effect of P446L GCKR and I148M PNPLA3 polymorphisms. Hepatology (Baltimore, Md). 2012;55(3):661-3.

60. Xia Y, Huang CX, Li GY, Chen KH, Han L, Tang L, et al. Meta-analysis of the association between MBOAT7 rs641738, TM6SF2 rs58542926 and nonalcoholic fatty liver disease susceptibility. Clin Res Hepatol Gastroenterol. 2019.

61. Abul-Husn NS, Cheng $X$, Li AH, Xin Y, Schurmann C, Stevis P, et al. A Protein-Truncating HSD17B13 Variant and Protection from Chronic Liver Disease. The New England journal of medicine. 2018;378(12):1096-106.

62. Koo BK, Joo SK, Kim D, Bae JM, Park JH, Kim JH, et al. Additive effects of PNPLA3 and TM6SF2 on the histological severity of non-alcoholic fatty liver disease. Journal of gastroenterology and hepatology. 2018;33(6):1277-85.

63. Stender S, Kozlitina J, Nordestgaard BG, Tybjaerg-Hansen A, Hobbs HH, Cohen JC. Adiposity amplifies the genetic risk of fatty liver disease conferred by multiple loci. Nature genetics. 2017;49(6):842-7.

64. Ma J, Hennein R, Liu C, Long MT, Hoffmann U, Jacques PF, et al. Improved Diet Quality Associates With Reduction in Liver Fat, Particularly in Individuals With High Genetic Risk Scores for Nonalcoholic Fatty Liver Disease. Gastroenterology. 2018;155(1):107-17.

65. Wang S, Song J, Shang X, Chawla N, Yang Y, Meng X, et al. Physical activity and sedentary behavior can modulate the effect of the PNPLA3 variant on childhood NAFLD: a casecontrol study in a Chinese population. BMC medical genetics. 2016;17(1):90.

66. Ashraf NU, Sheikh TA. Endoplasmic reticulum stress and Oxidative stress in the pathogenesis of Non-alcoholic fatty liver disease. Free radical research. 2015;49(12):140518. 
67. Steinberg D, Witztum JL. Oxidized low-density lipoprotein and atherosclerosis. Arteriosclerosis, thrombosis, and vascular biology. 2010;30(12):2311-6.

68. Binder CJ, Shaw PX, Chang MK, Boullier A, Hartvigsen K, Horkko S, et al. The role of natural antibodies in atherogenesis. Journal of lipid research. 2005;46(7):1353-63.

69. Yimin, Furumaki $H$, Matsuoka $S$, Sakurai $T$, Kohanawa $M$, Zhao $S$, et al. A novel murine model for non-alcoholic steatohepatitis developed by combination of a high-fat diet and oxidized low-density lipoprotein. Laboratory investigation; a journal of technical methods and pathology. 2012;92(2):265-81.

70. Walenbergh SM, Koek GH, Bieghs V, Shiri-Sverdlov R. Non-alcoholic steatohepatitis: the role of oxidized low-density lipoproteins. Journal of hepatology. 2013;58(4):801-10.

71. Bieghs V, van Gorp PJ, Walenbergh SM, Gijbels MJ, Verheyen F, Buurman WA, et al. Specific immunization strategies against oxidized low-density lipoprotein: a novel way to reduce nonalcoholic steatohepatitis in mice. Hepatology (Baltimore, Md). 2012;56(3):894903.

72. Binder CJ. Natural IgM antibodies against oxidation-specific epitopes. Journal of clinical immunology. 2010;30 Suppl 1:S56-60.

73. Hendrikx T, Watzenbock ML, Walenbergh SM, Amir S, Gruber S, Kozma MO, et al. Low levels of IgM antibodies recognizing oxidation-specific epitopes are associated with human non-alcoholic fatty liver disease. BMC medicine. 2016;14(1):107.

74. Tsimikas S, Brilakis ES, Lennon RJ, Miller ER, Witztum JL, McConnell JP, et al. Relationship of IgG and IgM autoantibodies to oxidized low density lipoprotein with coronary artery disease and cardiovascular events. Journal of lipid research. 2007;48(2):425-33.

75. Karvonen J, Paivansalo M, Kesaniemi YA, Horkko S. Immunoglobulin M type of autoantibodies to oxidized low-density lipoprotein has an inverse relation to carotid artery atherosclerosis. Circulation. 2003;108(17):2107-12.

76. Binder CJ, Horkko S, Dewan A, Chang MK, Kieu EP, Goodyear CS, et al. Pneumococcal vaccination decreases atherosclerotic lesion formation: molecular mimicry between Streptococcus pneumoniae and oxidized LDL. Nature medicine. 2003;9(6):736-43.

77. Ren S, Newby D, Li SC, Walkom E, Miller P, Hure A, et al. Effect of the adult pneumococcal polysaccharide vaccine on cardiovascular disease: a systematic review and meta-analysis. Open heart. 2015;2(1):e000247.

78. Ren S, Hure A, Peel R, D'Este C, Abhayaratna W, Tonkin A, et al. Rationale and design of a randomized controlled trial of pneumococcal polysaccharide vaccine for prevention of cardiovascular events: The Australian Study for the Prevention through Immunization of Cardiovascular Events (AUSPICE). American heart journal. 2016;177:58-65.

79. Suomela E, Oikonen M, Pitkanen N, Ahola-Olli A, Virtanen J, Parkkola R, et al. Childhood predictors of adult fatty liver. The Cardiovascular Risk in Young Finns Study. Journal of hepatology. 2016;65(4):784-90.

80. Denzer C, Thiere D, Muche R, Koenig W, Mayer H, Kratzer W, et al. Gender-specific prevalences of fatty liver in obese children and adolescents: roles of body fat distribution, sex steroids, and insulin resistance. The Journal of clinical endocrinology and metabolism. 2009;94(10):3872-81.

81. Barton M. Cholesterol and atherosclerosis: modulation by oestrogen. Curr Opin Lipidol. 2013;24(3):214-20.

82. Aldhoon-Hainerova I, Zamrazilova H, Hill M, Hainer V. Insulin sensitivity and its relation to hormones in adolescent boys and girls. Metabolism: clinical and experimental. 2017;67:908.

83. Couchepin C, Le KA, Bortolotti M, da Encarnacao JA, Oboni JB, Tran C, et al. Markedly blunted metabolic effects of fructose in healthy young female subjects compared with male subjects. Diabetes care. 2008;31(6):1254-6. 
84. Ter Horst KW, Serlie MJ. Fructose Consumption, Lipogenesis, and Non-Alcoholic Fatty Liver Disease. Nutrients. 2017;9(9).

85. Oertelt-Prigione S. The influence of sex and gender on the immune response. Autoimmunity reviews. 2012;11(6-7):A479-85.

86. Vos MB, Abrams SH, Barlow SE, Caprio S, Daniels SR, Kohli R, et al. NASPGHAN Clinical Practice Guideline for the Diagnosis and Treatment of Nonalcoholic Fatty Liver Disease in Children: Recommendations from the Expert Committee on NAFLD (ECON) and the North American Society of Pediatric Gastroenterology, Hepatology and Nutrition (NASPGHAN). Journal of pediatric gastroenterology and nutrition. 2017;64(2):319-34.

87. Vilar-Gomez E, Martinez-Perez Y, Calzadilla-Bertot L, Torres-Gonzalez A, Gra-Oramas B, Gonzalez-Fabian L, et al. Weight Loss Through Lifestyle Modification Significantly Reduces Features of Nonalcoholic Steatohepatitis. Gastroenterology. 2015;149(2):367-78.e5; quiz e14-5.

88. van der Windt DJ, Sud V, Zhang $H$, Tsung A, Huang $H$. The Effects of Physical Exercise on Fatty Liver Disease. Gene expression. 2018;18(2):89-101.

89. Utz-Melere M, Targa-Ferreira C, Lessa-Horta B, Epifanio M, Mouzaki M, Mattos AA. NonAlcoholic Fatty Liver Disease in Children and Adolescents: Lifestyle Change - a Systematic Review and Meta-Analysis. Annals of hepatology. 2018;17(3):345-54.

90. Knop C, Singer V, Uysal Y, Schaefer A, Wolters B, Reinehr T. Extremely obese children respond better than extremely obese adolescents to lifestyle interventions. Pediatr Obes. 2015;10(1):7-14.

91. Wiegand S, Keller KM, Lob-Corzilius T, Pott W, Reinehr T, Robl M, et al. Predicting weight loss and maintenance in overweight/obese pediatric patients. Horm Res Paediatr. 2014;82(6):380-7.

92. Reinehr T, Kleber M, Lass N, Toschke AM. Body mass index patterns over $5 \mathrm{y}$ in obese children motivated to participate in a 1-y lifestyle intervention: age as a predictor of longterm success. Am J Clin Nutr. 2010;91(5):1165-71.

93. Chinapaw MJ, Yildirim M, Altenburg TM, Singh AS, Kovacs E, Molnar D, et al. Objective and self-rated sedentary time and indicators of metabolic health in Dutch and Hungarian 10-12 year olds: the ENERGY-Project. PloS one. 2012;7(5):e36657.

94. Epstein LH, Paluch RA, Raynor HA. Sex differences in obese children and siblings in familybased obesity treatment. Obes Res. 2001;9(12):746-53.

95. Rijks J, Karnebeek K, van Dijk JW, Dorenbos E, Gerver WJ, Stouthart P, et al. Glycaemic Profiles of Children With Overweight and Obesity in Free-living Conditions in Association With Cardiometabolic Risk. Scientific reports. 2016;6:31892.

96. Ceriello A, Esposito K, Piconi L, Ihnat MA, Thorpe JE, Testa R, et al. Oscillating glucose is more deleterious to endothelial function and oxidative stress than mean glucose in normal and type 2 diabetic patients. Diabetes. 2008;57(5):1349-54.

97. Monnier L, Mas E, Ginet C, Michel F, Villon L, Cristol JP, et al. Activation of oxidative stress by acute glucose fluctuations compared with sustained chronic hyperglycemia in patients with type 2 diabetes. Jama. 2006;295(14):1681-7.

98. Webb M, Yeshua H, Zelber-Sagi S, Santo E, Brazowski E, Halpern Z, et al. Diagnostic value of a computerized hepatorenal index for sonographic quantification of liver steatosis. AJR American journal of roentgenology. 2009;192(4):909-14.

99. Zhang B, Ding F, Chen T, Xia LH, Qian J, Lv GY. Ultrasound hepatic/renal ratio and hepatic attenuation rate for quantifying liver fat content. World journal of gastroenterology. 2014;20(47):17985-92.

100. Borges VF, Diniz AL, Cotrim HP, Rocha HL, Andrade NB. Sonographic hepatorenal ratio: a noninvasive method to diagnose nonalcoholic steatosis. Journal of clinical ultrasound : JCU. 2013;41(1):18-25. 
101. Chauhan A, Sultan LR, Furth EE, Jones LP, Khungar V, Sehgal CM. Diagnostic accuracy of hepatorenal index in the detection and grading of hepatic steatosis. Journal of clinical ultrasound : JCU. 2016;44(9):580-6.

102. Cassinotto C, Boursier J, de Ledinghen V, Lebigot J, Lapuyade B, Cales P, et al. Liver stiffness in nonalcoholic fatty liver disease: A comparison of supersonic shear imaging, FibroScan, and ARFI with liver biopsy. Hepatology (Baltimore, Md). 2016;63(6):1817-27.

103. Eddowes PJ, Sasso M, Allison M, Tsochatzis E, Anstee QM, Sheridan D, et al. Accuracy of FibroScan Controlled Attenuation Parameter and Liver Stiffness Measurement in Assessing Steatosis and Fibrosis in Patients With Nonalcoholic Fatty Liver Disease. Gastroenterology. 2019;156(6):1717-30.

104. Ferraioli G, Calcaterra V, Lissandrin R, Guazzotti M, Maiocchi L, Tinelli C, et al. Noninvasive assessment of liver steatosis in children: the clinical value of controlled attenuation parameter. BMC gastroenterology. 2017;17(1):61.

105. Cho $\mathrm{Y}$, Tokuhara D, Morikawa $H$, Kuwae $\mathrm{Y}$, Hayashi $E$, Hirose $M$, et al. Transient Elastography-Based Liver Profiles in a Hospital-Based Pediatric Population in Japan. PloS one. 2015;10(9):e0137239.

106. Kwon YD, Ko KO, Lim JW, Cheon EJ, Song YH, Yoon JM. Usefulness of Transient Elastography for Non-Invasive Diagnosis of Liver Fibrosis in Pediatric Non-Alcoholic Steatohepatitis. Journal of Korean medical science. 2019;34(23):e165.

107. Tokuhara D, Cho Y, Shintaku H. Transient Elastography-Based Liver Stiffness AgeDependently Increases in Children. PloS one. 2016;11(11):e0166683.

108. Dong S, Zhan ZY, Cao HY, Wu C, Bian YQ, Li JY, et al. Urinary metabolomics analysis identifies key biomarkers of different stages of nonalcoholic fatty liver disease. World journal of gastroenterology. 2017;23(15):2771-84.

109. Svegliati-Baroni G, Pierantonelli I, Torquato P, Marinelli R, Ferreri C, Chatgilialoglu C, et al. Lipidomic biomarkers and mechanisms of lipotoxicity in non-alcoholic fatty liver disease. Free radical biology \& medicine. 2019.

110. Gorden DL, Myers DS, Ivanova PT, Fahy E, Maurya MR, Gupta S, et al. Biomarkers of NAFLD progression: a lipidomics approach to an epidemic. Journal of lipid research. 2015;56(3):722-36.

111. Mann JP, Vreugdenhil A, Socha P, Janczyk W, Baumann U, Rajwal S, et al. European paediatric non-alcoholic fatty liver disease registry (EU-PNAFLD): Design and rationale. Contemporary clinical trials. 2018;75:67-71. 
Summary 
The global prevalence of overweight and obesity in children and adults increased tremendously during the past decades, nowadays reaching epidemic proportions. Obesity is an important risk factor for the development of comorbidities affecting various organ systems, which can already start from a very young age in children. Among these comorbidities are cardiovascular disease, diabetes, sleep apnea, non-alcoholic fatty liver disease (NAFLD) and kidney disease. Moreover, children with obesity are likely to grow up to become adults with overweight or obesity and obesity-related health aberrations during childhood are strong predictors for disease at adult age. In this dissertation, the general health consequences of overweight and obesity in children were studied. Additionally, the aim of this dissertation was to gain more insight into determinants and risk factors of one of the comorbidities of overweight and obesity, namely hepatic steatosis and steatohepatitis. Finally, the effect of one year of the outpatient, familybased, multidisciplinary $\mathrm{COACH}$ lifestyle intervention on body mass index (BMI) z-score and comorbidities was evaluated.

In this dissertation, we have shown that comorbidities of obesity were already common at primary school age. Interestingly, most comorbidities were already as common in primary school children as in secondary school children. After one year of interdisciplinary, tailormade, lifestyle intervention, there was a significant decrease in BMI z-score (mean \pm SD:

$-0.18 \pm 0.40$ ), serum total cholesterol concentrations (median (interquartile range): -0.25 ($0.63 ; 0.10))$, LDL cholesterol concentrations $(-0.10(-0.50 ; 0.20))$ and triglyceride concentrations $(-0.05(-0.41 ; 0.19))$. The effect of the intervention on BMI z-score $(-0.25 \pm$ 0.32 vs. $-0.11 \pm 0.47)$, LDL cholesterol concentration $(-0.30(-0.70 ; 0.10)$ vs. -0.10 ($0.40 ; 0.30))$ and systolic blood pressure z-score $(-0.32 \pm 1.27$ vs. $0.24 \pm 1.35)$ was significantly greater in primary school children compared to secondary school children. Moreover, we have shown that glycemic profiles in free-living conditions in children with overweight and obesity also improved after one year of the $\mathrm{COACH}$ lifestyle intervention. We found a decrease in the duration of time that glucose concentrations were in the high normal range and a decrease in glycemic variability. In the children with a decrease in BMI z-score after one year of intervention, a decrease in median free-living glucose concentrations were associated with a decrease in systolic and diastolic blood pressure zscore. Combined, these findings reflect the need to start intervention as early as possible, and also to have attention for healthy lifestyle already early on in primary school to prevent the development of obesity and the accompanying health effects.

Non-alcoholic fatty liver disease is a common chronic liver disease of which the prevalence has strongly increased in the past decades alongside the obesity epidemic. The term 
NAFLD encompasses a spectrum of liver disorders ranging from hepatic steatosis, to steatohepatitis (NASH), liver fibrosis and cirrhosis. NAFLD can have severe consequences for the liver, but has also been associated with increased risk for developing diabetes type II and cardiovascular disease. At this time, the underlying pathophysiological mechanisms of NAFLD development and progression to more severe disease are still incompletely understood, but it is clear that it is the consequence of a complex interplay of multifactorial processes. Previous studies have shown histological differences in characteristics of NAFLD in children compared to adults, suggesting that underlying pathophysiological processes might also differ between children and adults. Therefore, it is important to study NAFLD development and progression not only in adults, but also in children. An increased insight in the children that might be at risk for progressive NAFLD can help in an early clinical recognition of these children and in the development of new treatment modalities.

In this dissertation, we have shown that the factors that are associated with the development of hepatic steatosis in children, as measured with the ultrasonographic hepatorenal index, are not the same as the factors that are associated with progression to steatohepatitis, as assessed with alanine aminotransferase (ALT) concentrations. Age, fasting glucose concentrations and C-reactive protein concentrations were associated with an increasing degree of hepatic steatosis. A higher degree of hepatic steatosis was associated with progression to steatohepatitis, just as the severity of overweight, male sex, serum triglyceride concentrations and serum ferritin concentrations. These results provide important insight into the factors that are associated with the consecutive NAFLD stages in children with overweight and obesity and demonstrate that it is important to separately study the different stages of NAFLD and the associated pathophysiological factors, in order to gain a better understanding of the progression along the disease spectrum.

Moreover, we found that children with a genetic predisposition (i.e. carrying a PNPLA3 and/or TM6SF2 risk allele) have higher ALT concentrations. Stratification for PNPLA3 and TM6SF2 genotype showed that the determinants of ALT concentrations differed between genotypes. Triglyceride concentrations were only associated with ALT levels in children carrying at least one PNPLA3 risk allele. Interestingly, even though triglyceride levels and HOMA-IR were identified as significant predictors of ALT levels in children carrying a PNPLA3 G allele, these children did not have elevated triglyceride levels or HOMA-IR, compared to children without a PNPLA3 risk allele. These results demonstrate that the factors involved in the development of NAFLD can differ between people with a different genetic background and cannot simply be generalized across populations. These 
differences are important to take into consideration from a clinical point of view, as we have demonstrated that high ALT levels can already be present with normal metabolic determinants in at-risk populations. Moreover, it is important to take these results into account in further scientific studies towards NAFLD, as the results might not be generalized across populations.

In previous studies oxidative stress has been associated with the pathophysiology of $\mathrm{NASH}$. Oxidative stress can lead to the formation of oxidized low-density lipoproteins (oxLDL) particles, which contribute to the formation of pro-inflammatory foam cells. In adults, oxLDL particles have been associated with an increased risk of cardiovascular disease, but also with NAFLD. Natural immunoglobulin M (IgM) antibodies can bind to oxidation-specific epitopes (OSE) that are present on oxLDL particles and protect against the harmful effects by blocking the formation of pro-inflammatory foam cells. An association between low levels of OSE-specific antibodies and NAFLD and cardiovascular disease was previously also described in adults. In this dissertation, we have shown for the first time in children that low concentrations of antibodies against OSE on oxLDL were associated with higher liver enzyme levels in obese children, suggesting that low OSEspecific IgM antibodies might play a role in the etiology of disturbed liver health in children. Associations between the OSE-specific antibodies and cardiovascular parameters were not found in our pediatric populations.

In summary, the results described in this dissertation have shown that comorbidities of childhood obesity are already common in primary school children. Moreover, our results show that one year of outpatient, multidisciplinary lifestyle intervention had a positive effect on BMI z-score and comorbidities (total cholesterol, LDL cholesterol and triglyceride concentrations in the total group of children and systolic blood pressure in primary school children only), with a larger effect in primary school children compared to secondary school children. Finally, we have evaluated which parameters are associated with the development of hepatic steatosis and progression to steatohepatitis in children and have shown that these determinants differ between children with a different genetic predisposition.

The findings in this dissertation underline the need to start prevention and lifestyle interventions as early as possible to prevent the development of (more severe) obesity and the accompanying health aberrations. NAFLD in children remains an important research topic in the future. A better understanding of the determinants of (progressive) NAFLD in children, in combination with appropriate imaging techniques, will help in 
achieving a better clinical recognition of children that are at-risk for progressive liver disease, or have already developed NAFLD. Moreover, since treatment options for NAFLD are currently limited, increased insight in the underlying pathophysiological mechanisms can contribute to the identification of new treatment options for NAFLD. 
Samenvatting 
De wereldwijde prevalentie van overgewicht en obesitas bij kinderen en volwassenen is de afgelopen decennia sterk toegenomen. Obesitas is een belangrijke risicofactor voor het ontwikkelen van comorbiditeit in verschillende orgaansysteem, waaronder hart- en vaatziekten, diabetes, slaapapneu, niet-alcoholische leververvetting (NAFLD) en nierziekten. Deze comorbiditeiten van obesitas kunnen ook al op jonge leeftijd bij kinderen ontstaan. De aanwezigheid van obesitas-gerelateerde aandoeningen op kinderleeftijd is een belangrijke voorspeller voor de aanwezigheid van ziekte op volwassenleeftijd. Daarnaast hebben kinderen met obesitas ook een grotere kans om als volwassene overgewicht of obesitas te hebben.

In dit proefschrift zijn de algemene comorbiditeiten van overgewicht en obesitas bij kinderen onderzocht. Daarnaast was het doel van het onderzoek dat is beschreven in dit proefschrift om meer inzicht te krijgen in de determinanten en risicofactoren van leversteatose en steatohepatitis, een van de comorbiditeiten van overgewicht en obesitas. Tot slot werd ook het effect van een jaar deelname aan de interdisciplinaire $\mathrm{COACH}$ leefstijlinterventie op BMI z-score en comorbiditeit geëvalueerd.

Het onderzoek dat is beschreven in dit proefschrift laat zien dat comorbiditeit bij kinderen met obesitas al veel voorkomt op basisschoolleeftijd. De prevalentie van gezondheidsrisico's en comorbiditeit verschilde nauwelijks tussen kinderen met obesitas van basisschoolleeftijd en middelbare schoolleeftijd. Na een jaar deelname aan de interdisciplinaire $\mathrm{COACH}$ leefstijlinterventie was er een significante afname in BMI z-score (gemiddelde \pm standaarddeviatie: $-0.18 \pm 0.40$ ), totaal cholesterol concentraties (mediaan (interkwartiel range): $-0.25(-0.63 ; 0.10))$, LDL cholesterol concentraties $(-0.10(-0.50 ; 0.20))$ en triglyceride concentraties $(-0.05(-0.41 ; 0.19))$. Het effect van de interventie op BMI zscore $(-0.25 \pm 0.32$ vs. $-0.11 \pm 0.47)$, LDL cholesterol concentraties $(-0.30(-0.70 ; 0.10)$ vs. $0.10(-0.40 ; 0.30)$ en systolische bloeddruk $z$-score $(-0.32 \pm 1.27$ vs. $0.24 \pm 1.35)$ was significant groter in kinderen van basisschoolleeftijd vergeleken met kinderen van middelbare schoolleeftijd.

Daarnaast was er bij kinderen met overgewicht en obesitas na een jaar COACH leefstijlinterventie ook een verbetering van glycemische profielen in het dagelijks leven zichtbaar. Er was namelijk een afname van de duur van hoog-normale glucosewaardes en een afname van glycemische variabiliteit. Bij de kinderen waarbij er na een jaar leefstijlinterventie sprake was van een afname in BMI z-score, was een afname van de mediane glucosewaarde geassocieerd met een afname in systolische en diastolische bloeddruk z-score. Deze resultaten benadrukken het belang van vroege preventie en interventies die het ontstaan van obesitas en de gezondheidsgevolgen daarvan beogen te voorkomen. 
Niet-alcoholische leververvetting (NAFLD) is een veelvoorkomende chronische leverziekte waarvan de prevalentie de afgelopen decennia sterk is toegenomen. Deze toename viel samen met het optreden van de obesitasepidemie. De term NAFLD omvat een spectrum van leveraandoeningen, variërend van leversteatose, tot steatohepatitis (NASH), leverfibrose en -cirrose. NAFLD kan ernstige gevolgen hebben voor de lever zelf, maar was in eerder onderzoek ook geassocieerd met een verhoogd risico op het ontwikkelen van type 2 diabetes en hart- en vaatziekten. Op dit moment is de onderliggende pathofysiologie, van het ontstaan en de progressie van NAFLD, nog niet helemaal opgehelderd. Het is wel duidelijk dat er sprake is van een complexe wisselwerking tussen verschillende multifactoriële processen. Daarnaast hebben eerdere studies verschillen laten zien tussen de histologische kenmerken van NAFLD bij kinderen vergeleken met volwassenen, wat suggereert dat de onderliggende pathofysiologische processen mogelijk ook verschillen tussen kinderen en volwassenen. Het is daarom van belang om het ontstaan en de progressie van NAFLD ook te onderzoeken in kinderen. Een verbeterd inzicht in welke kinderen een verhoogd risico hebben op het ontstaan van (progressieve) NAFLD kan bijdragen aan de vroege herkenning van deze kinderen en een rol spelen in het ontwikkelen van nieuwe behandelopties.

De resultaten beschreven in dit proefschrift laten zien dat de factoren die geassocieerd zijn met het ontstaan van leversteatose bij kinderen, zoals gemeten met de echografische lever-nier ratio, niet hetzelfde zijn als de factoren die zijn geassocieerd met de progressie naar steatohepatitis, zoals beoordeeld door middel van alanine aminotransferase (ALAT) concentraties. Leeftijd, nuchtere glucose concentraties en C-reactieve proteïne concentraties waren namelijk geassocieerd met leversteatose. Een ernstigere mate van leversteatose was daarnaast geassocieerd met progressie naar steatohepatitis, net als de ernst van het overgewicht, mannelijk geslacht, serum triglyceride concentraties en serum ferritine concentraties. Deze resultaten geven een belangrijk inzicht in de factoren die geassocieerd zijn met de verschillende NAFLD-stadia bij kinderen met overgewicht en obesitas en laten zien dat het belangrijk is om deze verschillende stadia en de geassocieerde pathofysiologie afzonderlijk te onderzoeken om beter inzicht te krijgen in de progressie van NAFLD.

De resultaten beschreven in dit proefschrift laten ook zien dat kinderen met een genetische predispositie (kinderen die drager zijn van een of twee PNPLA3 en/of TM6SF2 risico-allelen) hogere ALAT concentraties hebben. Een vergelijking van kinderen met de verschillende PNPLA3 en TM6SF2 genotypen liet zien dat de determinanten van ALAT concentraties verschillen tussen deze genotypen. Triglyceride concentraties waren alleen 
geassocieerd met ALAT concentraties bij kinderen met minstens één PNPLA3 risico-allel. Ondanks dat triglyceride concentraties en HOMA-IR significante voorspellers waren voor ALAT concentraties bij kinderen met een PNPLA3 risico-allel, hadden deze kinderen echter geen verhoogde triglyceride concentraties of HOMA-IR vergeleken met kinderen zonder een PNPLA3 risico-allel.

Deze resultaten laten zien dat de factoren die zijn betrokken bij het ontstaan van NAFLD kunnen verschillen tussen mensen met een verschillende genetische achtergrond en daarom niet zomaar gegeneraliseerd kunnen worden naar hele populaties. Vanuit een klinisch oogpunt is het van belang om rekening te houden met deze genetische verschillen, omdat hoge ALAT concentraties bij kinderen met een hoog genetisch risico al aanwezig kunnen zijn terwijl de metabole parameters normaal zijn. Daarnaast is belangrijk om deze bevindingen ook in wetenschappelijk onderzoek naar NAFLD mee te nemen, omdat de resultaten mogelijk niet gegeneraliseerd kunnen worden naar hele populaties.

In eerdere studies is ook oxidatieve stress geassocieerd met de pathofysiologie van NAFLD. Oxidatieve stress kan leiden tot het ontstaan van geoxideerde lage-dichtheid lipoproteïne (oxLDL) deeltjes, die bijdragen aan de vorming van pro-inflammatoire schuimcellen. Eerder onderzoek bij volwassenen heeft laten zien dat oxLDL deeltjes geassocieerd zijn met een verhoogd risico op hart- en vaatziekten, maar ook op NAFLD. Immunoglobuline M (IgM) antilichamen kunnen binden aan oxidatie-specifieke epitopen (OSE) die aanwezig zijn op de oxLDL deeltjes. Hierdoor wordt de vorming van proinflammatoire schuimcellen geblokkeerd en beschermen IgM antilichamen tegen de negatieve effecten van oxLDL.

Bij volwassenen werd eerder al een associatie gevonden tussen lage concentraties IgM antilichamen tegen OSE en het optreden van NAFLD en hart- en vaatziekten. De resultaten in dit proefschrift laten nu voor het eerst zien dat lage concentraties van antilichamen tegen OSE ook bij kinderen met obesitas geassocieerd zijn met hogere concentraties leverenzymen. Deze resultaten suggereren dat lage concentraties van OSE-specifieke IgM antilichamen mogelijk een rol spelen in de etiologie van NAFLD in kinderen. In onze populatie kinderen met obesitas werden geen associaties gevonden tussen de IgM antilichamen tegen OSE en cardiovasculaire parameters.

Samenvattend laten de resultaten beschreven in dit proefschrift zien dat comorbiditeiten van obesitas al veel voorkomen op jonge leeftijd. Daarnaast hebben we laten zien dat een jaar poliklinische, interdisciplinaire leefstijlinterventie een positief effect heeft op de BMI z-score en gezondheid van kinderen met obesitas (namelijk een afname van totaal cholesterol, LDL cholesterol en triglyceride concentraties in de totale groep kinderen en 
ook een afname van de systolische bloeddruk z-score in kinderen van basisschoolleeftijd). Het effect van de interventie was groter bij kinderen van basisschoolleeftijd, vergeleken met kinderen van middelbare schoolleeftijd. Tot slot is in dit proefschrift geëvalueerd welke parameters geassocieerd zijn met het ontstaan van leversteatose en progressie naar steatohepatitis bij kinderen en laten zien dat deze determinanten verschillen tussen kinderen met een verschillende genetische predispositie.

De bevindingen in dit proefschrift benadrukken het belang van preventie en vroege interventies om de ontwikkeling van (ernstigere) obesitas en de daarmee geassocieerde gezondheidsgevolgen te voorkomen. NAFLD bij kinderen blijft een belangrijk onderwerp voor verder wetenschappelijk onderzoek. Een beter begrip van de determinanten van de verschillende stadia van NAFLD bij kinderen kan, in combinatie met beeldvormende technieken, bijdragen aan een betere klinische herkenning van kinderen met een hoog risico op (progressieve) NAFLD. Daarnaast kan meer inzicht in de onderliggende pathofysiologische mechanismen bijdragen aan nieuwe behandelopties, die op dit moment nog erg beperkt zijn. 
Valorisation 
In addition to the scientific relevance of the studies performed in this thesis, it is important to also discuss the relevance besides science, for instance the relevance in clinical settings, but also the societal and economical relevance. The valorisation of the acquired knowledge about comorbidities of overweight and obesity in children, with a special focus on non-alcoholic fatty liver disease (NAFLD), and the effect of multidisciplinary lifestyle intervention on BMI z-score and comorbidities, are further elaborated in this chapter.

An unhealthy lifestyle and obesity are important causes of morbidity and mortality worldwide. As we have shown in this thesis, and was previously also shown by others (1, 2 ), the development of comorbidities is already seen at a young age. In addition to physical health consequences, children with overweight and obesity are more likely to suffer from psychological problems, like depression, low self-esteem and decreased quality of life (3). Moreover, children with overweight or obesity are likely to become adults with obesity and the associated comorbidities are also likely to track into adulthood (4). Finally, in addition to the effects of long-term obesity on the health of the individual, there is also a substantial economical and societal burden.

A previous German study has estimated that the expected excess lifetime healthcare costs were higher ( 3.7 and 5 times higher, respectively in men and women) in adults that were overweight or obese as a child compared to adults that had a normal weight as a child (5). Another previous German study has estimated that a BMI higher than $40 \mathrm{~kg} / \mathrm{m}^{2}$ in adults is associated with healthcare costs twice as high as in adults with a normal weight. Additional costs for society, mainly due to reduced work productivity, sick leave and longterm incapacity was also twice as high in these adults with morbid obesity (6).

The fact that obesity and the associated diseases like diabetes, cardiovascular disease and NAFLD can have serious consequences on the health of the individual, but also constitutes an economic burden for society, underlines the need for prevention and effective interventions. The results of one of the studies in this dissertation have shown that there was a clinically relevant decrease in BMI z-score and health outcomes improve after one year of on-going, family-based, multidisciplinary lifestyle intervention in children with obesity at the Centre for Overweight Adolescent and Children's Healthcare (COACH). This study also showed that these positive effects, on both BMI z-score and health outcomes, are more pronounced in younger children. At this time, a cost-benefit analysis of the $\mathrm{COACH}$ program has not yet been performed, however it is expected that the aforementioned approach contributes to reduced healthcare costs of the treatment of childhood obesity. A recent societal cost-benefit analysis of a two-year multidisciplinary 
lifestyle intervention in children with overweight and obesity (in Arnhem, the Netherlands) estimated that the economic benefits of such an intervention are 4-7 higher than the costs (7). In this study, the lifetime economic benefit of lifestyle intervention in children was estimated to be between 11384 and 19120 euros per individual, mainly due to decreased current and future healthcare costs and an improved quality of life due to a decreased disease burden.

In current society, living a healthy lifestyle and losing weight can be a difficult goal to achieve, since unhealthy products and services are easily available. Creating a society in which healthy options are accessible and affordable for everyone, requires combined efforts from stakeholders at multiple levels, like different health care professionals (for instance physicians, dieticians and psychologists), schools, sports clubs, supermarkets and (local) governments.

In the $\mathrm{COACH}$ lifestyle intervention, an interdisciplinary team of health care providers works together with various stakeholders to provide a tailor-made intervention for each child and their family. The aim is to achieve and maintain a healthy lifestyle by making small, step-by-step lifestyle changes that can be maintained throughout a long period of time. In order to achieve this, children and their families receive individual guidance, focusing on personalized lifestyle improvements. Of course, intervention programs could be considered costly, because long-term care is provided. In order to limit the costs of this intervention, the intervention plan is tailored to the needs of each child and their family, rather than providing the same plan for all children. In this approach, focus points that are identified before the intervention (and might be adjusted during the intervention) are prioritized together with the family, in order to provide the appropriate guidance at different moments throughout the intervention. Moreover, during the past decade, the $\mathrm{COACH}$ program has evolved into an elaborate collaboration with different local stakeholders, such as youth health care, dieticians, a family coach, but also the local municipality, fruit and vegetable farmers and supermarkets. Together with these partners, children are stimulated to lead a healthier lifestyle by participating in sports and healthy food-related activities on a regular basis, in addition to the individual guidance by health care providers. Working together with multiple societal partners that are also motivated in helping to create a healthier living environment for children, contributes to the commitment and success of the intervention.

Based on the experiences of $\mathrm{COACH}$ and seven other regional lifestyle interventions in the Netherlands, aimed at guiding children with obesity and their families, a national approach for the care for these children was developed recently ('landelijk model 
ketenaanpak voor kinderen met overgewicht en obesitas'). This model provides a basis and step-wise approach for health care providers and societal partners that aim to accomplish a local network collaboration to provide care for children with overweight and obesity. With this national model, the same approach can be expanded to other regions, but also to a broader target group (i.e. children across the entire overweight - morbid obesity spectrum). Expanding the lifestyle interventions that are currently already available for children is necessary to ensure that all children and their families can receive help in achieving and maintaining a healthy lifestyle. Prevention of (increasing) overweight and obesity and the associated health consequences will have a positive effect on the physical and psychosocial health of the individual, but will also help in decreasing the societal burden of obesity (e.g. by decreasing costs and increasing work productivity).

In this dissertation we have not only studied the comorbidities of childhood obesity in general, but also studied non-alcoholic fatty liver disease specifically. The prevalence of NAFLD has strongly increased the past decades, alongside the obesity epidemic. As was discussed in the previous chapters of this dissertation, hepatic steatosis and steatohepatitis (NASH) will progress to liver cirrhosis and liver failure in a subgroup of patients, possible necessitating a liver transplantation. Also, NAFLD patients have an increased risk of developing hepatocellular carcinoma, illustrating that the disease can have severe consequences for the health of the individual. Finally, NAFLD has been associated with an increased risk for developing cardiovascular disease and diabetes type II. These findings underline the importance of early recognition of NAFLD patients.

A recent study showed that between 2006 and 2016, the number of NAFLD-related liver transplantations increased by $170 \%$ in the United States, making it the second leading cause of liver transplantation (8). Currently, weight loss through lifestyle improvements is the recommended treatment for children with NAFLD. Therefore, lifestyle interventions, such as the $\mathrm{COACH}$ program, can contribute to decreasing disease severity and preventing progression to severe disease states that might require a liver transplant. However, since weight loss is currently the only proven treatment for NAFLD in children, there is an urgent need for other treatment options to prevent progression to severe stages of the disease, especially in patients that experience difficulties in achieving and maintaining weight loss.

NAFLD is often asymptomatic and most patients do not have specific symptoms until they have progressed to the severe stages of the disease spectrum. Currently, the gold standard for diagnosing and staging non-alcoholic fatty liver disease is a liver biopsy. Since 
this is an invasive procedure with a small risk of severe complications, liver biopsies are not suitable for screening the large number of people that are at-risk for developing NAFLD. As mentioned previously in this dissertation, other diagnostic modalities are less suitable for screening purposes or have limited sensitivity for staging NAFLD. Therefore, an increased insight in the children that are at risk for (progressive) NAFLD can help in an early clinical recognition of these children, in which prevention of further weight gain and metabolic disturbances is very important. In the studies in this dissertation, we have evaluated parameters that are associated with the different stages of NAFLD in children with overweight and obesity. An increased insight into the factors associated with NAFLD development and progression can also help in the development of new treatment modalities.

In addition to affecting the individual, the health consequences associated with NAFLD also have significant economic consequences. It was recently estimated that the direct annual economic burden of NAFLD in 4 European countries (Germany, France, Italy and the United Kingdom) was 35 billion euros. When combined with the societal costs of the loss of quality adjusted life years due to NAFLD and its complications, the annual economic burden was estimated to be 228 billion euros. Costs due to a loss in work productivity were not taken into account in this estimation. Also, this study did not investigate whether the economic burden was dependent or independent of obesity (9). It is to be expected that early recognition of patients that have developed NAFLD, increased insight in the risk factors for (fast) progressive disease and new treatment options contribute to a decreased burden on the healthcare system, including liver transplantations, and a decreased societal economic burden. 


\section{References}

1. Skinner AC, Perrin EM, Moss LA, Skelton JA. Cardiometabolic Risks and Severity of Obesity in Children and Young Adults. The New England journal of medicine. 2015;373(14):130717.

2. Reinehr T, Wiegand S, Siegfried W, Keller KM, Widhalm K, I'Allemand D, et al. Comorbidities in overweight children and adolescents: do we treat them effectively? Int J Obes (Lond). 2013;37(4):493-9.

3. Rankin J, Matthews L, Cobley S, Han A, Sanders R, Wiltshire HD, et al. Psychological consequences of childhood obesity: psychiatric comorbidity and prevention. Adolescent health, medicine and therapeutics. 2016;7:125-46.

4. Juhola J, Magnussen CG, Viikari JS, Kahonen M, Hutri-Kahonen N, Jula A, et al. Tracking of serum lipid levels, blood pressure, and body mass index from childhood to adulthood: the Cardiovascular Risk in Young Finns Study. The Journal of pediatrics. 2011;159(4):584-90.

5. Sonntag D, Ali S, Lehnert T, Konnopka A, Riedel-Heller S, Konig HH. Estimating the lifetime cost of childhood obesity in Germany: Results of a Markov Model. Pediatr Obes. 2015;10(6):416-22.

6. Yates N, Teuner CM, Hunger M, Holle R, Stark R, Laxy M, et al. The Economic Burden of Obesity in Germany: Results from the Population-Based KORA Studies. Obesity facts. 2016;9(6):397-409.

7. Harchaoui S, Vellenga I, Spit W, Steeg van de L, Peters N. Maatschappelijke effecten van aanpak van obesitas - Kosten en baten van Gezond Onderweg! 2018.

8. Wong RJ, Aguilar M, Cheung R, Perumpail RB, Harrison SA, Younossi ZM, et al. Nonalcoholic steatohepatitis is the second leading etiology of liver disease among adults awaiting liver transplantation in the United States. Gastroenterology. 2015;148(3):547-55.

9. Younossi ZM, Blissett D, Blissett R, Henry L, Stepanova M, Younossi Y, et al. The economic and clinical burden of nonalcoholic fatty liver disease in the United States and Europe. Hepatology (Baltimore, Md). 2016;64(5):1577-86. 
Dankwoord 
Na 4 jaar met plezier aan mijn proefschrift te hebben gewerkt, is het nu tijd voor het laatste hoofdstuk. Een proefschrift schrijven doe je niet alleen, dus bij deze wil ik graag iedereen bedanken die een bijdrage heeft geleverd aan het eindresultaat.

Allereerst wil ik natuurlijk graag mijn promotoren bedanken. Anita en Jogchum, jullie passie voor jullie werk en wetenschappelijk onderzoek is aanstekelijk! Ik kijk terug op een periode waarin ik heel veel heb geleerd en jullie mij hebben gestimuleerd het beste uit mezelf te halen. De afgelopen jaren heb ik niet alleen veel geleerd over het doen van wetenschappelijk onderzoek, maar heb ik mijzelf op allerlei vlakken kunnen ontwikkelen. Waar ik me vier jaar geleden wel eens afvroeg of dat 'onderzoek doen' me wel ging lukken, ligt hier nu toch een proefschrift voor me waar ik trots op ben. 'Fake it 'til you become it', toch? Veel dank ook voor jullie vertrouwen om ons onderzoek en COACH op allerlei verschillende plekken en voor verschillende publieken te mogen vertegenwoordigen. Daarnaast waardeer ik ook jullie persoonlijke interesse heel erg en dat er af en toe ook gewoon tijd was voor een praatje over van alles en nog wat, tijdens congres-avonden, zomerborrels of gewoon even tussendoor. Ik kijk er naar uit om de komende jaren verder samen te werken.

Prof. dr. Zimmermann, beste Luc, dank voor de mogelijkheid om mijn promotieonderzoek binnen de vakgroep Kindergeneeskunde uit te kunnen voeren.

Daarnaast wil ik graag alle $\mathrm{COACH}$ patiënten en hun gezinnen bedanken. Met veel plezier heb ik mijn werk als onderzoeker de afgelopen jaren gecombineerd met het begeleiden van kinderen en hun gezinnen bij het maken van een gezondere leefstijl.

De beoordelingscommissie, Prof. dr. Kremers, Prof. dr. Feskens, Dr. Koek, Prof. dr. Schrauwen en Prof. dr. Verkade wil ik graag bedanken voor het kritisch beoordelen van mijn proefschrift.

Veel dank ook aan alle co-auteurs. Jullie betrokkenheid bij de verschillende studies en feedback op de manuscripten daarvan hebben mij veel geleerd en inhoudelijk veel bijgedragen aan de inhoud van dit proefschrift.

Ik had het geluk dat ik onderdeel mocht zijn van verschillende teams en vakgroepen. Jullie hebben allemaal, op verschillende manieren, bijgedragen aan mijn proefschrift en de fijne tijd de afgelopen jaren. 
Allereerst, het $\mathrm{COACH}$-team, van nu en de afgelopen jaren. Anita, Pauline, Elly B., Elly C., Jesse, Elke, Lisanne, Nadine, Cara, Ellen, Anne, Ida, Joyce, Chantal, Ronny en Rianne bedankt voor de samenwerking de afgelopen jaren. Elly $C$. en Jesse, als zorgcoördinator en $\mathrm{COACH}$-promovendus van het eerste uur, een extra woord van dank voor jullie aanstekelijke enthousiasme voor $\mathrm{COACH}$ en hulp tijdens mijn eerste stappen binnen $\mathrm{COACH}$ en daarna. Ik ben met veel plezier onderdeel geweest van $\mathrm{COACH}$, het voelde vanaf het begin als een warm bad. Ik heb van jullie allemaal geleerd. Op naar het 10 -jarig jubileum en nog vele daarna! Anita, ik hoop dat al je dromen voor de toekomst van de zorg voor kinderen met overgewicht en obesitas uitkomen. Met een alsmaar groeiend team en netwerk van enthousiaste mensen gaat dat zeker lukken!

De onderzoekers van de Kindergeneeskunde wil ik graag allemaal bedanken voor de koffie- en lunch- breaks, overlegmomentjes tussendoor en gezellige momenten buiten de werkvloer. Jesse, Yvon, Elke, Marieke en Kim, allemaal al met ervaring op zak toen ik begon, bedankt voor jullie hulp en delen van jullie ervaring. De 'nieuwe generatie', Johanna, Gabrielle, Mark, Dorien, Lisanne, Minela, Eduardo, Britt en Judith, veel succes met jullie onderzoek. Op naar nog vele promotiefeestjes de komende jaren!

Ook veel dank aan iedereen van de Phun-groep van nu en de afgelopen jaren. Ondanks dat ik maar een klein deel van mijn tijd bij jullie aanwezig was, voelde ik me toch altijd onderdeel van de groep! Ik heb veel geleerd van al jullie onderzoeken tijdens de wekelijkse besprekingen en op congressen. Daarnaast was er ook altijd veel gezelligheid tijdens etentjes, sportieve momenten en gewoon even bijkletsen op de gang. Succes allemaal met jullie onderzoek!

Maurice en Maud, veel dank voor jullie hulp bij een deel van de labanalyses in mijn proefschrift. Als ik dat zelf had gedaan, hadden de resultaten er waarschijnlijk heel anders uit gezien.

Daarnaast wil ik alle studenten bedanken die zich de afgelopen jaren voor $\mathrm{COACH}$ hebben ingezet.

Prof. dr. Robben, beste Simon, veel dank voor de hulp bij het verrichten en beoordelen van alle leverecho's. Ik ben de tel kwijtgeraakt, maar het waren er zeker veel. Ik vond het leuk om het maken van de leverecho's als extra vaardigheid te leren tijdens mijn promotietraject. 
De collega's van de vakgroep Kindergeneeskunde wil ik graag bedanken voor hun interesse in ons onderzoek. Veel dank ook aan de verpleegkundigen van de PICU, G3 en het PSA-team voor hun inzet bij het verrichten van alle infusen, onderzoeken en metingen.

Anne de Bruijn, Yolanda Verhaegen en Tamara Cauberg, bedankt voor jullie hulp bij het inplannen van afspraken, regelen van inloggegevens en bestellingen enzovoorts.

Natuurlijk wil ik ook mijn lieve vrienden en familie bedanken voor jullie interesse en gezelligheid.

Michelle, al vriendinnen vanaf de brugklas en nu reserve-paranimf vanaf je vakantieadres. Etentjes, wijntjes, musicals of buitenlandse tripjes. Het maakt niet uit, het is altijd gezellig. Op naar nog vele!

Lieve Marjolijn, jij bent voor altijd in mijn hart!

Nandi, Merel en Anne, borrelen, dansjes, fietsen, filmavondjes, India-herinneringen ophalen en de rest waren altijd een fijne afleiding. Ook al zien we elkaar niet zo vaak met $z^{\prime} n$ vieren, het is altijd gezellig. Limmie, ijsjes, escape-roomen, last-minute etentjes en Rendang van je mama waren ook altijd goed.

Johanna en Cara, bedankt voor jullie gezelligheid op het werk en daarbuiten. Een beetje gedeeld (leed)vermaak om mijn dubieuze records of Cara's Belgische vocabulair, Johanna's beste Kardashian Fun Facts en etentjes waren altijd een leuke momentjes tussendoor. Johanna, onze (mini)-Ironman-avonturen waren een goede afleiding tijdens het laatste half jaar van mijn promotietraject.

Putri, wat fijn dat jij vandaag mijn paranimf wilt zijn. Ook al zien we elkaar wat minder vaak nu je niet meer in Maastricht woont, de gezelligheid van 5 jaar in onze studentenpenthouse is er nog altijd. Vakanties naar Cuba en Santorini waren goede relaxmomenten. Volgende keer een reis naar Bali met jou als privégids?

Kevin, mijn grote broer. Paranimf is natuurlijk een titel die echt bij jou past, of is in ieder geval een goed excuus om een nieuw pak te kopen. Jouw nuchtere kijk op dingen (behalve als je weer eens een traumahelikopter wilt bellen voor een snee in je vinger), komen vast van pas mochten de zenuwen toe slaan voor mijn verdediging. Ik hoop dat je goed hebt geoefend op de uitspraak van de stellingen. Maud, jij ook bedankt voor je interesse en het ondersteunen van Kevin bij deze zware taak. 
Lieve oma, in het weekend bij jou een kopje koffie drinken is altijd gezellig. Ik ben blij dat je er vandaag bij bent. Opa Wim en oma Nieke zouden vast ook trots zijn.

Papa en mama, jullie hebben je volgens mij wel vaker afgevraagd wat ik de afgelopen vier jaar nou eigenlijk heb gedaan, behalve die dinsdagavond-poli's waarna we altijd even moesten bellen. Nou, dit is dus het resultaat. Bedankt voor jullie onvoorwaardelijke steun en vertrouwen in mijn kennis en kunde. Dank jullie wel voor alles! 
About the author 
Kylie Karnebeek was born in Heerlen on April 13, 1990. She graduated from secondary school at Sintermeertencollege in Heerlen in 2008. She studied Medicine at Maastricht University from 2009 until 2015. During the last year of her Medicine study, she performed her research internship at the Centre for Overweight Adolescent and Children's Healthcare (COACH) under the supervision of Dr. Anita Vreugdenhil. After graduating, she continued working as a physician and PhD Candidate at $\mathrm{COACH}$ and the NUTRIM School of Nutrition and Translational

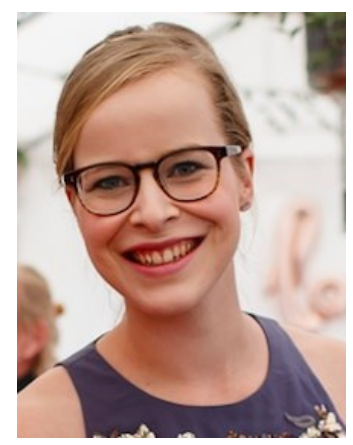
Research in Metabolism. Under the supervision of Dr. Anita Vreugdenhil and Prof. dr. Jogchum Plat she studied health aberrations in children with overweight and obesity, with a focus on non-alcoholic fatty liver disease. During her time as a PhD Candidate, she presented her study results at several national and international conferences. The most important scientific results are described in this dissertation. From January 2020 she will start working at the department of Pediatrics in the Viecuri Hospital in Venlo. 
List of publications 
Karnebeek, K., Thapar, S., Willeboordse, M., Schayck van, O.C.P \& Vreugdenhil, A.C.E. Comorbidities in primary versus secondary school children with obesity and responsiveness to lifestyle intervention, The Journal of Clinical Endocrinology \& Metabolism, Volume 104, Issue 9, September 2019.

De Nooijer, A., Vreugdenhil, A.C.E., Karnebeek, K., Hasselt van, P. \& Fuchs, S. A narrative review of factors associated with the development and progression if non-alcoholic fatty liver disease. Gastrohep, Volume 1, Issue 4, July 2019.

Rijks, J.M., Karnebeek, K., Dijk van, J.W., Dorenbos, E., Gerver, W.J.E., Stouthart, P., Plat, J. \& Vreugdenhil, A.C.E. Glycaemic Profiles of Children With Overweight and Obesity in Freeliving Conditions in Association With Cardiometabolic Risk. Scientific Reports, August 2016.

Rijks, J.M., Vreugdenhil, A.C.E., Dorenbos, E., Karnebeek, K., Joris, P., Berendschot, T., Mensink, R.P. \& Plat, J. Characteristics of the retinal microvasculature in association with cardiovascular risk markers in children with overweight, obesity and morbid obesity. Scientific Reports, November 2018.

Karnebeek, K., Willeboordse, M., Schayck van, O.C.P \& Vreugdenhil, A.C.E. Leefstijlinterventie voor kinderen met obesitas: hoe jonger, hoe beter. Nederlands Tijdschrift voor Geneeskunde, geaccepteerd voor publicatie.

Karnebeek, K., Rijks, J.M., Dorenbos, E., Gerver, W.J.E., Plat, J. \& Vreugdenhil, A.C.E. Changes in free-living glycemic profiles after 12 months of lifestyle intervention in children with overweight and obesity. Submitted.

Karnebeek, K., Vreugdenhil, A.C.E., Hendrikx, T., Plat, J., Oligslaeger, Y. \& Shiri-Sverdlov, R. Low levels of antibodies against oxidation specific epitopes in oxLDL are associated with parameters reflecting NAFLD and metabolic health in children. Submitted.

Karnebeek, K., Plat, J., Robben, S. \& Vreugdenhil, A.C.E. Factors associated with increasing degrees of hepatic steatosis and progression to steatohepatitis in children with overweight or obesity. Submitted.

Karnebeek, K., Plat, J. \& Vreugdenhil, A.C.E. Genotype-specific determinants of alanine aminotransferase levels in children with overweight and obesity. Submitted.

Karnebeek, K., Plat, J., Rijks, J.M., Mensink, R.P. \& Vreugdenhil, A.C.E. Predictors of arterial stiffness in children with overweight, obesity and morbid obesity are sex-specific. Submitted. 
